\title{
REGULATION OF THE ERYTHROPOIETIN GENE
}

P. H. Maxwell

Corpus Christi College

D. Phil. Thesis

Michaemas 1994

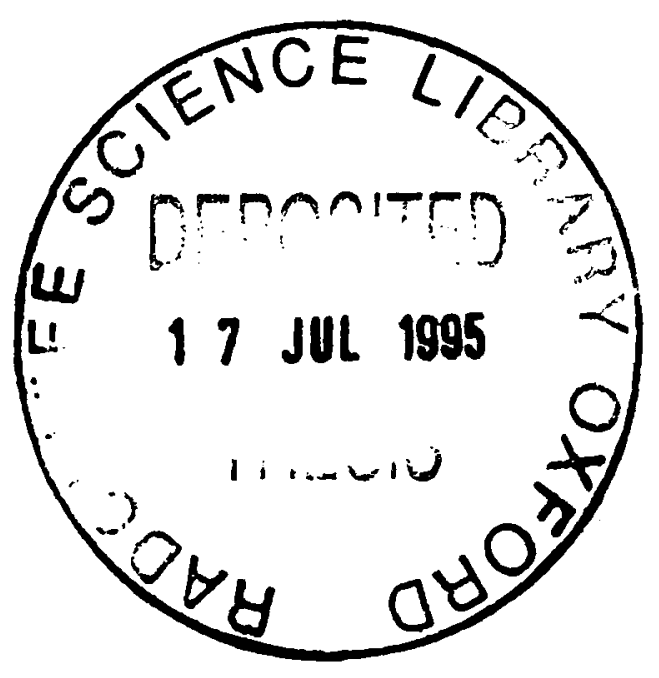




\section{Regulation of the erythropoietin gene}

\section{ABSTRACT}

Erythropoietin (Epo) is a circulating hormone which regulates red blood cell production, and hence oxygen-carrying capacity. Three striking features of Epo gene expression are that it shows high level induction in response to hypoxia, that it is tightly tissue-restricted and that it is deficient in most forms of renal damage. Each of these aspects has been investigated in this thesis.

Chapter 3 concerns the hypoxic-sensing system which induces Epo gene expression via an enhancer element in cultured hepatoma cells. Transient transfection was used to examine a range of cell-lines, which did not produce Epo, for the presence of this oxygen-sensing system. It was demonstrated that such a system is widespread in mammalian cells. It is probable that it is involved in regulating the expression of other genes in response to hypoxia.

This widespread oxygen-sensing mechanism contrasts with the tightly tissue-restricted expression of the Epo gene. Transgenic experiments described in Chapters 4 and 5 provide data concerning the DNA sequences involved in this tissue specificity. Sequence from the mouse Epo locus was then used to direct expression of a marker protein, SV40 T antigen, to Epo-expressing cells. This led to identification of the Epo-producing cells in the kidney (Chapter 5) and liver (Chapter 6). In both organs a fibroblast-like population expresses the gene; the Ito cells in the liver and the Type 1 interstitial cells in the kidney. In the liver hepatocytes also produce Epo.

Further studies of Epo gene expression would be facilitated by the availability of Epoproducing cell lines. SV40 T antigen is a viral oncogene, and expression of this protein was used as a proliferative stimulus in vivo. The effects of this, and attempts to isolate these cells form the subject of Chapter 7. Chapter 7 also describes experiments in which renal injury was combined with visualisation of individual Epo-producing cells.

A homologous recombination at the Epo locus resulted in an allele which expressed the hormone at a greatly reduced level. The phenotype of animals homozygous for this allele is described in Chapter 8. Experiments designed to produce further modification of the Epo locus by this method, and to assess its utility in modifying the mouse genome are also described.

Submitted for D. Phil., Michaelmas 1994 Patrick Maxwell, Corpus Christi College 


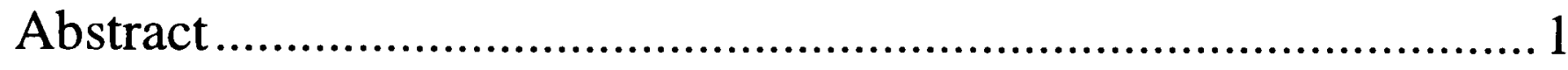

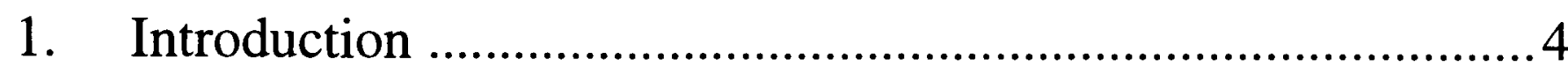

1.1. Erythropoietin regulation - an historical and physiological

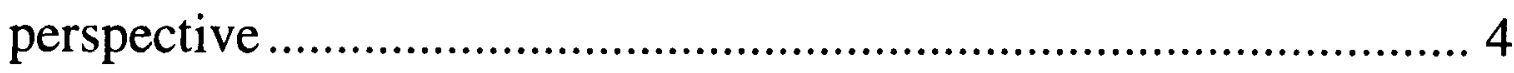

1.2. Regulation of the Epo gene - molecular biology .......................... 24

1.3. Major features of Epo regulation .............................................. 33

1.4. Mechanisms of transcriptional control ....................................... 33

1.5. Principal issues addressed in this thesis .................................... 37

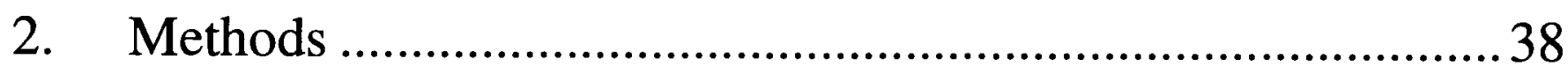

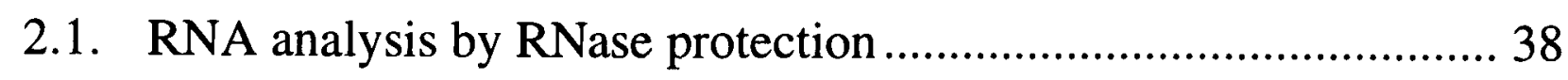

2.2. Maintenance of established cell lines ........................................ 43

2.3. Transient transfection of DNA .................................................. 44

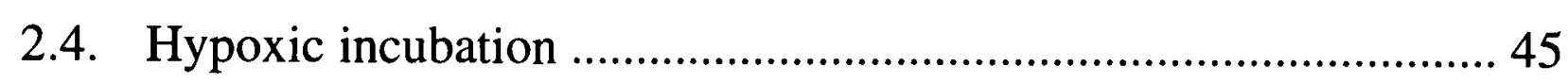

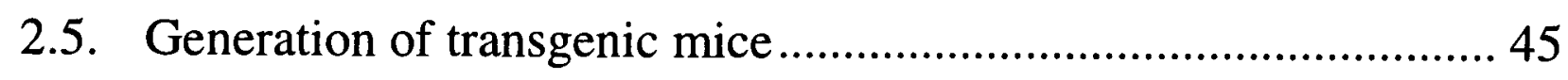

2.6. Breeding and maintenance of mice ........................................ 47

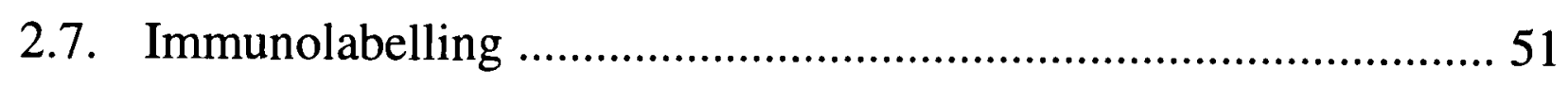

3. Evidence for a widespread oxygen-sensing mechanism in mammalian cells ........................................................ 57

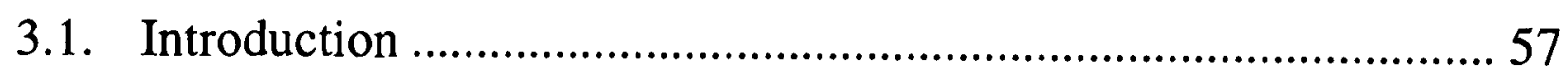

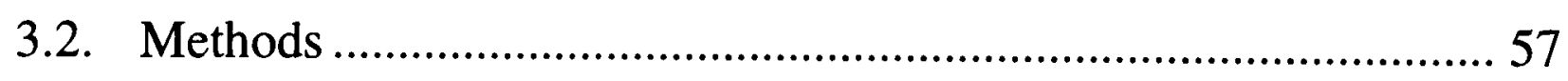

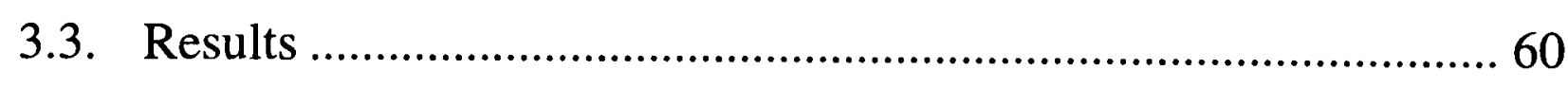

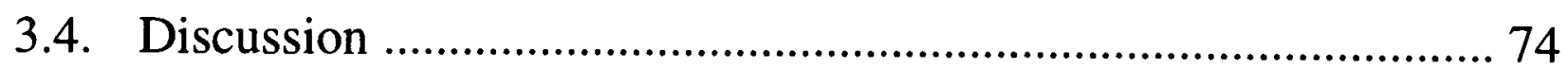

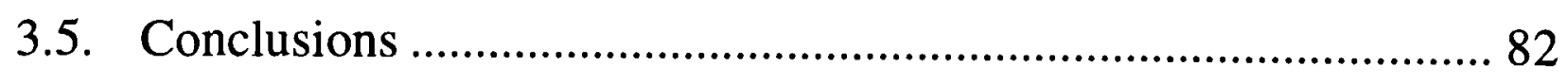

4. Determination of mouse Epo sequence sufficient to direct appropriate expression of a marker gene ........................... 84

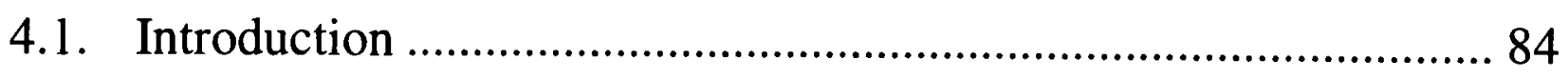

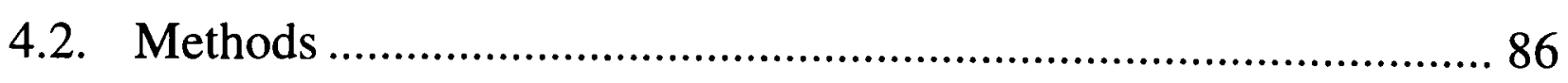

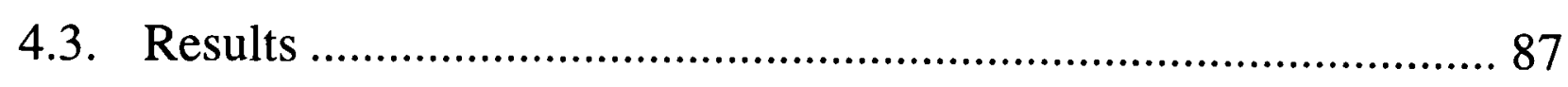

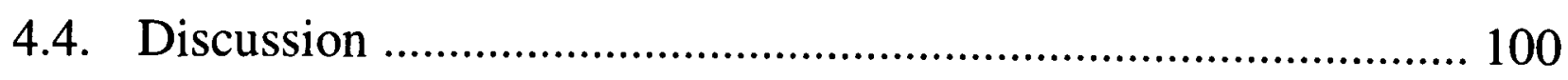

5. Identification of the renal Epo-producing cells .................. 105

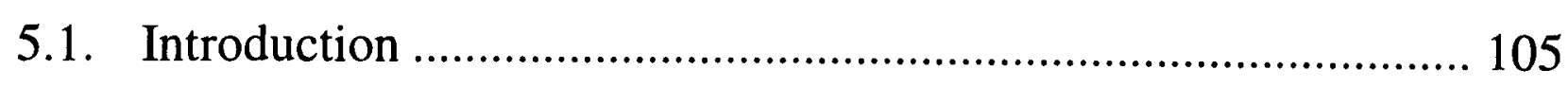

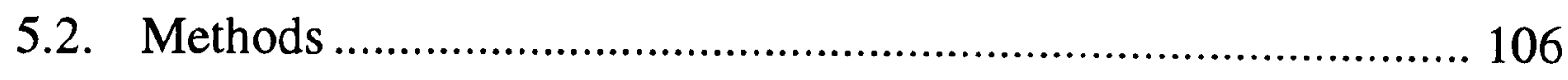

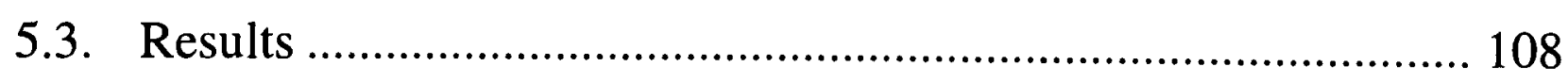

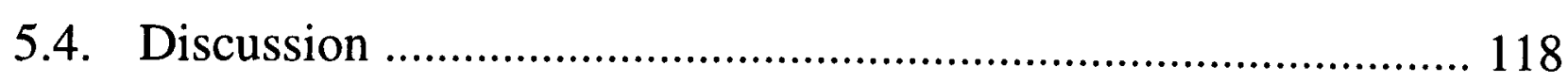


6. Identification of hepatic Epo-producing cells.

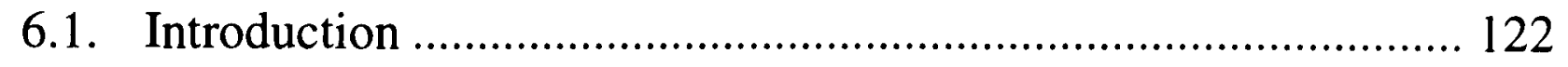

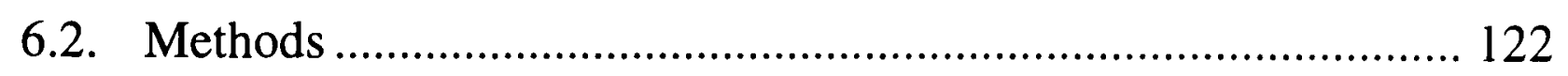

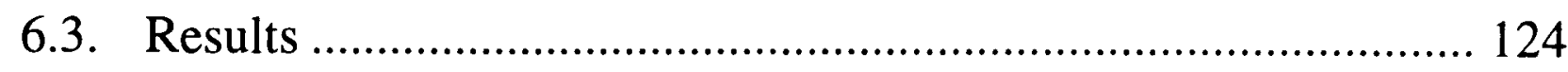

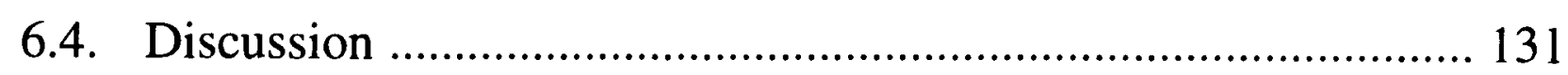

7. Biology of renal Epo-producing cells ................................... 136

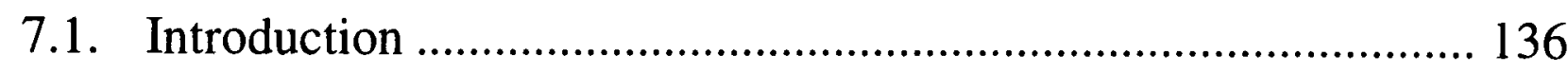

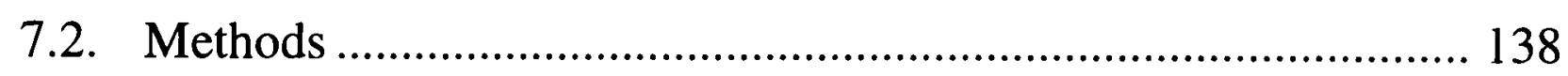

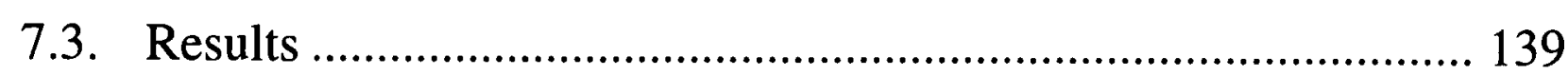

7.4. General discussion ............................................................ 166

8. Homologous recombination at the Epo locus...................... 168

8.1. Introduction ....................................................................... 168

8.2. Evidence for HR in the Epo-TAg ${ }^{\mathrm{H}}$ line .................................. 171

8.3. Timing of the recombination event ......................................... 174

8.4. Phenotype of Epo-TAg ${ }^{\mathrm{H}}$ mice. ............................................... 175

8.5. Degree of isogenicity in the Epo-TAg experiment .................... 178

8.6. Rate of HR at the Epo locus. ................................................ 180

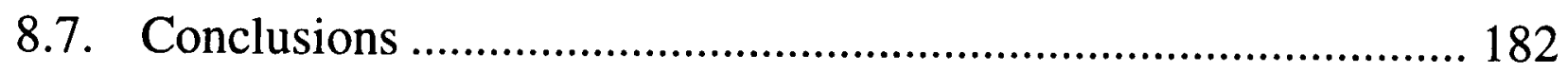

9. Concluding remarks ................................................. 184

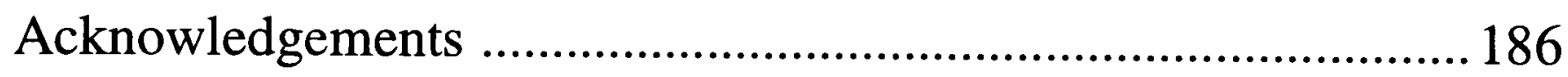

Appendices ................................................................... 187

1. The Epo 3' enhancer in mouse and man .................................... 187

2. Transgene sequence and PCR diagnosis ................................. 188

3. Cosmid clones and mapping the Epo locus ............................... 190

4. Chinese hamster Epo sequence and riboprobe ........................... 191

5. 3' Flanking sequence in various mouse strains ......................... 192

6. Principal publications arising from work in this thesis .............. 193

References ................................................................ 194 


\section{Introduction}

\subsection{ERYTHROPOIETIN REGULATION - AN HISTORICAL AND PHYSIOLOGICAL PERSPECTIVE}

It is now almost ninety years since it was first suggested that the formation of red blood cells was under the influence of a circulating regulator. The existence of such a hormonal regulator, named erythropoietin (Epo), is now established. This thesis is concerned with the regulation of expression of the Epo gene. The rationale for the experimental work, and the design of these experiments, has relied heavily on accumulated physiological data concerning the regulation of erythropoiesis. This introductory chapter will therefore summarize some of the key observations concerning Epo. Central observations are firstly that Epo production appears to be principally determined by the oxygen availability of blood reaching the kidney, and secondly that regulation appears to be primarily at the level of transcription of the Epo gene.

\subsubsection{Transport of oxygen in the blood; the erythron}

Individual cells have several essential requirements; the supply of energy substrate and oxygen for respiration being two of the most fundamental. This is achieved through the circulation of a complex fluid, blood, into the close vicinity of almost all cells. This constant traffic also facilitates supplies of raw materials, waste product removal, immune surveillance and communication between parts of the organism which are physically separated. The transport of sufficient oxygen presents a considerable challenge because of its poor solubility. In order to achieve transport of sufficient oxygen, respiratory pigments appear at an early stage of evolution in invertebrates such as worms and gastropods, initially in the coelomic fluid or circulating plasma. In some invertebrates, notably segmented worms, specialised haemoglobin-containing cells are observed, as modifications of lymphocyte-like cells (Jordan 1933). All vertebrates have haemoglobin-containing cells in the blood. In normal human adults there are about $2 \times 10^{13}$ red cells, perhaps best regarded as a circulating organ, the erythron, with a total volume of about 2 litres. The importance of the haemoglobin they contain is clearly demonstrated by the fact that in normal human blood about $99 \%$ of the total oxygen is bound to haemoglobin. Somewhat less than $1 \%$ of the circulating human erythron is replaced each day from the bone marrow by the formation of new red cells (erythropoiesis). 


\subsubsection{Homeostasis of the erythron}

The size of the erythron is kept relatively constant by altering the rate of erythropoiesis; thus in response to anaemia the rate of erythropoiesis can be increased approximately tenfold, and in response to an increase in red cell mass it is reduced. In addition, erythropiesis can be stimulated by lack of oxygen. Thus humans at altitude develop an increase in erythropoiesis (Faura et al. 1969; Milledge and Cotes 1985), and this is also observed in laboratory animals.

A reasonable hypothesis would be that the variable governing the rate of erythropoiesis is the available oxygen content of the blood, which is dependent both on the concentration of haemoglobin and the arterial partial pressure of oxygen. Thus if the inspired oxygen concentration falls, or the haemoglobin concentration falls, an increase in erythropoiesis is observed. If a proportion of the haemoglobin is rendered unavailable for oxygen transport by exposure to carbon monoxide, this has a similar effect to anaemia. Blood oxygen availability would also be influenced by the affinity of haemoglobin for oxygen; and (rare) individuals with an abnormal haemoglobin resulting in increased affinity for oxygen have an increase in the size of the erythron (Adamson and Finch 1975).

\subsubsection{Detection of changes in blood oxygen availability}

A sensing mechanism operated by the arterial partial pressure of oxygen would not be influenced by the haemoglobin content of the blood or the haemoglobin affinity. In order to sense anaemia or altered haemoglobin affinity, it would be necessary to detect the partial pressure of oxygen either in venous blood or in a tissue, i.e. following the extraction of a proportion of the oxygen from the blood. This variable would be dependent on the oxygen content of the arterial blood, but would also be influenced by local oxygen consumption.

Early observations in whole animals suggested that this was indeed the case. Manoeuvres expected to decrease oxygen consumption, such as starvation and hypophysectomy, resulted in suppression of erythropoiesis and conversely treatment with thyroxine increased erythropoiesis. In each of these circumstances, in response to a putative fall in tissue oxygenation, the formation of red blood cells is increased. Blood oxygen availability is therefore enhanced, completing a negative feedback loop. Although many other variables have been found to have some influence on the rate of erythropoiesis, and the effects of starvation on erythropoiesis are more complex than simply reducing oxygen consumption, the major regulatory system appears to be the operation of such a feedback loop. 
One possible mechanism for this would be that blood oxygen availability directly influenced the rate of erythropoiesis by the bone marrow as originally proposed by Miescher (Miescher 1893). However, this is not the case.

\subsubsection{Evidence for control by a circulating factor, erythropoietin}

The increased level of erythropoiesis is not a direct effect of reduced blood oxygen content but (at least in mammals) is mediated by a circulating glycoprotein hormone, named erythropoietin, acting on the erythropoietic tissues (bone marrow and spleen). The existence of such a factor was first suggested by Carnot and Deflandre (Carnot and Deflandre 1906); however their experimental observations could not be repeated by others and their proposal remained controversial for many years.

The first conclusive demonstration of a humoral substance was provided by Reissmann (Reissmann 1950). He used parabiotic rats in which one was exposed to hypoxia and the other to room air; both animals developed erythroid hyperplasia of the bone marrow. Further supportive evidence came from the observation that a patient with regional hypoxia of the lower body due to a patent ductus arteriosus had erythroid hyperplasia of his well-oxygenated sternal bone marrow (Stohlmann et al. 1954). The presence of a circulating factor was incontrovertibly demonstrated by showing that plasma from anaemic animals would stimulate erythropoiesis when injected into normal animals (Erslev 1953; Plzak et al. 1955).

Erythropoietins from different mammalian species show a high degree of cross-reactivity in both immunoassays and bioasssays. Outside mammals less is known about the existence of circulating erythropoietic factors; there is, however some evidence for such a factor in birds (Rosse and Waldmann 1966), frogs (Rosse et al. 1963), and fish (Zanjani et al. 1969). The cross-reactivity of these factors with the mammalian erythropoietins would appear to be limited on the basis of serum transfer experiments.

\subsubsection{Measurement of Epo}

Much of our knowledge about the regulation of erythropoiesis comes from the measurement of Epo in biological fluids, and to a lesser extent in tissues. This was first performed on the basis of the serum-transfer experiments described above. The sample to be tested was injected into a test animal and the rate of erythropoiesis was assessed. Over a short time interval major advances were made in improving these in vivo bioassays. The use of Fe59 incorporation into erythrocytes as a specific measure of erythropoiesis was an important developement (Plzak et al. 1955). A further refinement was to ensure that the recipient 
animal's baseline level of erythropoiesis was low. Several methods were used to achieve this; starvation, hypophysectomy and post-hypoxic or post-transfusion polycthaemia (reviewed in Krantz and Jacobson 1970). Another important step was the development of a system of standardisation to allow the comparison of results from different laboratories initially the response of fasted rats to $5 \mu \mathrm{mol}$ cobalt was used as an international unit of comparison, but subsequently a batch of crude sheep Epo extract was set aside as Standard A; later this was replaced by a crude preparation from human urine (Standard B) (Cotes and Bangham 1966). These assays were both expensive and labour intensive. Furthermore, it remained difficult to detect low concentrations of the hormone reliably; to the extent that there was uncertainty whether Epo was present under conditions of normal haematocrit and oxygenation.

In addition to in vivo assays, in vitro bioassays were also developed (for review see Jelkmann 1992). Although these potentially offered higher sensitivity, they were usually not entirely specific for Epo.

\subsubsection{Purification of Epo, cloning of the Epo gene and development of radioimmunoassays.}

In 1977 Miyake et al. succeeded in purifying a few milligrammes of Epo to homogeneity from over 2000 litres of urine collected from human subjects with aplastic anaemia (Miyake et al. 1977). This provided a high quality immunogen for preparing antibodies to Epo and material for preparing radiolabelled tracer, thus allowing the development of specific and sensitive radioimmunoassays for the hormone (Sherwood and Goldwasser 1979; Cotes 1982; Garcia et al. 1982; McGonigle et al. 1984). These assays were up to 100 more times sensitive than in vivo bioassays.

Furthermore, using the purified Epo it was then possible to obtain amino acid sequences of tryptic fragments of the protein, and hence to screen genomic and cDNA libraries and isolate the human Epo gene (Jacobs et al. 1985; Lin et al. 1985). It was found to be a single copy gene encoding a 166 amino acid protein with a 27 amino acid leader sequence. This accounted for only $18.4 \mathrm{kDa}$, the remainder of the $30.4 \mathrm{kDa}$ human Epo molecule being formed by glycosylation. Expression of the human Epo gene in mammalian cells (which was necessary for correct glycosylation) led to the availability of recombinant human Epo for research and therapeutic applications. Subsequently, simian (Lin et al. 1986) and murine (McDonald et al. 1986; Shoemaker and Mitsock 1986) Epo genes were succesfully cloned. It then became possible to study the underlying molecular biology of Epo production. 


\subsubsection{Structure of the gene and species comparisons}

In addition to coding sequence, the Epo locus must also include cis-acting control elements. Analysis of the non-coding sequence at the Epo locus is therefore likely to yield important information concerning regulation of the Epo gene. Since evolution produces a steady mutational drift in sequence, important deductions can often be drawn from comparing sequence from different species. Regions that are functionally insignificant, or where the function has changed, are expected to show substantial divergence; in contrast functionally important regions will be relatively well preserved. Furthermore, comparison with the sequence of known genes and their regulatory sequences may provide functional information.

The initial Epo clones included limited 5' and 3' flanking sequence. In each species the gene structure is similar with five exons and four introns. There is no TATA or CAAT box. Comparison of the coding sequences between primate and rodent showed extensive homology as expected from the species cross-reactivity in bioassays. In non-coding sequence there are also areas of conservation. These regions of substantial homology are thus likely to be of functional importance. Thus there is a $140 \mathrm{bp} \mathrm{G+C}$ region with a very high degree of homology (>90\%) lying immediately 5 ' to the gene. Other areas of homology include a further $100 \mathrm{bp} 5^{\prime}$ to this region, the first intron, the 5' and 3' untranslated regions (McDonald et al. 1986; Shoemaker and Mitsock 1986) and a region lying $120 \mathrm{bp} 3$ ' to the polyadenylation site (Pugh et al. 1991).

More recently, with the publication of further sequence in the $5^{\prime}$ flanking region, 6 further areas of $5^{\prime}$ homology between the mouse and human genes have been identified, over a distance of $3 \mathrm{~kb} 5$ ' to the previously reported mouse clone (which extended only $0.4 \mathrm{~kb} 5$ to the transcription start site) (Galson et al. 1993). These regions of 25 to $250 \mathrm{bp}$ are 70 to 80 $\%$ identical, suggesting that they are functionally important. These homologous regions are summarised in Figure 1.1. 


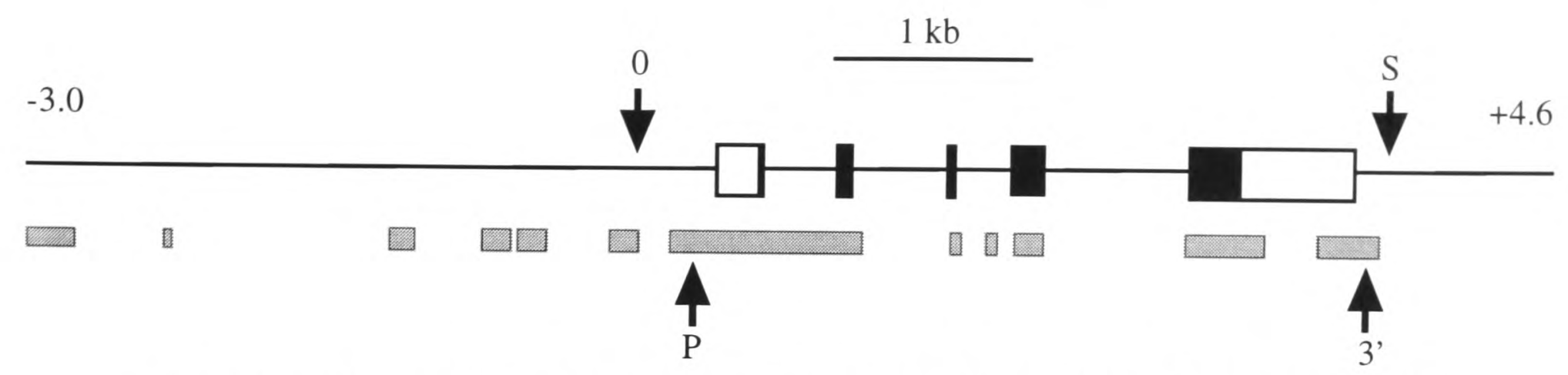

Figure 1.1 - The organisation of the mouse Epo gene is shown with the five exons. Coding sequence is solid and untranslated regions are shown as open boxes. $3.0 \mathrm{~kb} 5^{\prime}$ and $4.6 \mathrm{~kb} 3^{\prime}$ to the Xbal site (taken as 0) and the four introns are shown as a line. Comparison with the human sequence reveals significant homology in the regions indicated by stippled boxes beneath the DNA. The 3' enhancer region investigated in the mouse and human is indicated by 3'. The promoter region, which is not clearly defined, is indicated by $P$. The tgEPO4 transgene of Semenza consisted of human DNA extending across a region corresponding to 0 to $S$ in the mouse. Expression of this transgene was hypoxically inducible in the liver.

\subsubsection{Physiology of Epo production}

Under normal physiological conditions radioimmunoassays demonstrate that there is a low, but detectable, level of Epo in the blood. This level is about 4-30 mIU/ml (Cotes 1982), corresponding to a few pmol/l.

In humans with primary polycythaemia the level of Epo is low or normal, but is seldom completely suppressed (Cotes 1982; Cotes et al. 1986). Similarly, when humans exposed to high altitude return to sea-level they have an inappropriately high circulating red cell mass and reduced, but detectable Epo levels (Milledge and Cotes 1985). Thus there is some evidence for a constitutive level of Epo expression which is not suppressed when oxygen delivery is increased.

In response to a small stimulus, such as donating a single unit of blood, there is a measurable increase in Epo levels and erythropoiesis, although the absolute level of Epo may remain in the normal range (Kickler and Spivak 1988; Lorentz et al. 1991). This, combined with the demonstration that polycythaemia reduces Epo levels provides evidence that the feedback loop regulating Epo production in response to changes in blood oxygen content is functional under normal physiological conditions.

If mice are passively immunised with rabbit antiserum to Epo, erythropoiesis is suppressed (Schooley and Garcia 1962). Similarly, if mice are immunised with human Epo (Kurtz et al. 1989) they become severely anaemic. It therefore also appears that there is an absolute requirement for the low basal levels of Epo to maintain normal erythropoiesis. This suggests that there is less redundancy than with other haematopoietic growth factors and cytokines, and that Epo is more similar to other classical hormones such as insulin, parathormone and growth hormone. 
With anaemia or hypoxia there is a rapid, high amplitude, response with peak serum levels up to a thousand-fold higher than those in normal subjects. There is a measurable response to an acute stimulus within thirty minutes (Kurtz et al. 1988), and it takes about six to twenty four hours to achieve a peak response in rodents, and somewhat longer in humans. Importantly, the response to anaemia can be suppressed by exposure to hyperbaric oxygen, as predicted if the sensed variable is blood oxygen availability (Necas and Neuwirt 1969).

The increased levels of Epo in response to changes in blood oxygen availability appear to be entirely due to increased synthesis of the hormone. In particular, there is no evidence that the clearance of Epo from plasma varies in response to blood oxygen availability. The Epo response is almost entirely prevented by inhibitors of RNA and protein synthesis (Schooley and Mahlmann 1972b), suggesting that it is dependent on de novo transcription of the gene and new protein synthesis. This suggested that the increased Epo levels in response to changes in oxygen availability are occurring substantially at the level of gene transcription.

An overwhelming body of evidence implicates the kidney in the operation of the erythropoietic feedback loop; as discussed below, a restricted population of cells in this organ is the major site of Epo synthesis in adult mammals. Although the liver is the dominant site of production in the foetus, and makes a significant contribution in adult life, there appears to be little expression in other organs.

\subsubsection{Kidney as the major site of Epo synthesis}

Determination of the site of Epo synthesis relied initially on the surgical removal of individual organs from rats, followed by a stimulus to Epo production. After hypophysectomy, thyroidectomy, splenectomy, adrenalectomy and gonadectomy an erythropoietic response to haemorrhage could still occur. Following removal of seven-eighths of the liver, or removal of the spleen, adrenals, pancreas, stomach or intestines, a response to injected cobalt was still observed. However, following removal of the kidneys no response to cobalt or haemorrhage was obtained (Jacobson et al. 1957). Moreover, blood taken 10 - 12 hours following nephrectomy did not contain detectable Epo by in vivo bioassay. These experiments suggested an essential role for the kidney in the control of erythropoiesis.

For some years it remained possible that the kidneys were producing a precursor of Epo or a converting enzyme in response to hypoxia, rather than synthesising the hormone itself. The demonstration of Epo in serum-free perfusate from isolated rabbit kidneys disproved 
this hypothesis. The subsequent extraction of Epo from hypoxic kidney (Fried et al. 1982) and the demonstration that the kidney contained Epo mRNA (Beru et al. 1986; Bondurant and Koury 1986) confirmed that the kidneys actually produced Epo. In support of the central importance of the kidney in adult erythropoiesis is the well-established clinical observation that humans with chronic renal failure are usually anaemic with a relative deficiency of Epo (Caro et al. 1979; Eschbach 1989) and this anaemia is successfully treated with recombinant human Epo (Winearls et al. 1986; Eschbach et al. 1987).

The amount of Epo mRNA in kidney from normal animals is low and detection requires sensitive techniques, i.e. RNase protection or Northern blotting following poly A selection (Koury et al. 1988a). In response to haemorrhage or cobalt in rats (Beru et al. 1986), haemorrhage in mice (Bondurant and Koury 1986) and hypoxia (Schuster et al. 1987) there is a rapid, high amplitude accumulation of Epo mRNA in the kidneys of intact animals. The accumulation of renal Epo mRNA correlated with the Epo content of the kidney and, with a short lag time, the serum Epo level (Schuster et al. 1987).

"Nuclear run-on" of Epo mRNA transcripts was observed using kidney cell nuclei from cobalt-treated or anaemic-hypoxic animals but not with preparations from control animals, demonstrating that the level of regulation in the intact kidney is at least partially at the level of transcription (Schuster et al. 1989). This supported the previous observation that pretreatment with actinomycin D reduced the Epo response to subsequent hypoxic exposure (Schooley and Mahlmann 1972b). Following brief hypoxic exposure ( 2 hours at 22,000 ft), the levels of serum Epo continue to rise for a few hours (Schooley and Mahlmann 1972b); this continued rise is not sensitive to Actinomycin $\mathrm{D}$ and is presumably due to the relatively slow disappearance of mRNA once formed.

More recently it has been demonstrated that isolated perfused kidneys could respond to reduced oxygenation of the perfusate with increased Epo mRNA production (Ratcliffe et al. 1990) or increased Epo in the perfusate (Pagel et al. 1991). These experiments demonstrate not only that the kidney can produce Epo but also that it can detect an alteration in blood oxygen content. It remains possible that Epo production from the kidney is also influenced by oxygen-sensing at remote sites; however there is no direct evidence for this. Some support for the idea is the observation that controlled reduction of blood-flow to one kidney (using a clip) is a relatively poor stimulus to Epo production (Pagel et al. 1989). However, this may be a rather different stimulus from arterial hypoxemia or anaemia; for instance it 
will decrease glomerular filtration, renal tubular work and hence oxygen consumption. If a remote site were involved it is relevant that complete denervation of one kidney does not influence basal expression or inducible expression of the Epo gene (Eckardt et al. 1992a).

In view of the high level of inducibility that is observed under optimal conditions in isolated perfused kidney preparations it seems likely that the hypoxic control mechanism for renal Epo production resides solely in the kidney.

\subsubsection{Identification of the site of synthesis within the kidney}

Clearly it is important to understand how changes in blood oxygen availability are sensed at a cellular and molecular level. In order to do this, it has been an important goal to identify which cells within the kidney express the Epo gene, both under normal and hypoxic or anaemic circumstances. To date, various methods have been used to address this issue, but the information obtained has been incomplete, and (to some extent) conflicting. Since the identification of these cells forms a major part of this thesis, the data gained from previous studies is discussed in some detail.

\section{Morphological studies}

Observations of increased granularity in the juxta-glomerular apparatus in mice subject to repeated phlebotomy led to the suggestion that these cells might be the site of Epo production (Osnes 1958). However, in subsequent studies it became clear that juxta-glomerular granularity was not meaningfully correlated with Epo production, and was influenced by many other factors (reviewed in Krantz and Jacobson 1970).

\section{Immuno-localisation of the protein}

Attempts to localise Epo production within the kidney using antibodies raised against the hormone have been confusing; this is probably because the hormone is not stored at the site of synthesis, and because it is a small enough molecule to be filtered by the glomerulus, and then reabsorbed by the tubule. Additionally, earlier studies used rather crude immunogens to produce polyclonal antisera, which were probably of low specificity for Epo.

One early report, using a rabbit antiserum to partially purified sheep Epo to label sheep kidney sections suggested a glomerular site (Fisher et al. 1965). Another, using rabbit antiserum to pure human Epo to label sections of anaemic rat kidney, suggested mesangial cells (Fukushima et al. 1990). Tubular cells have also been labelled using Epo antibodies (Maxwell et al. 1990). Using a murine monoclonal antibody to human Epo, rather weak immunoreactivity was demonstrated in a peritubular location in kidneys from anaemic (but 
not control) mice, in cells identified as capillary endothelial cells on the basis of shape (Suzuki and Sasaki 1990). These cells were not numerous and were located in the deep cortex.

\section{Localisation of Epo mRNA}

Epo mRNA does accumulate rapidly in the whole kidney in response to hypoxia. Clearly localisation of these transcripts at a cellular level should provide important information about the site of Epo production. Two techniques have been used to address this; firstly separating the kidney into fractions, and secondly in situ hybridisation on tissue sections. The use of separation techniques has been limited. Schuster et al. demonstrated Epo mRNA in the tubular, as opposed to the glomerular fraction of disrupted renal cortex (Schuster et al. 1987).

In situ hybridisation studies have provided conflicting information. One group has suggested that Epo mRNA is present in tubular epithelium in mice subjected to hypobaric hypoxia (Maxwell et al. 1990). However, these studies used a ${ }^{32} \mathrm{P}$ labelled oligonucleotide (45 bp in length), which may have impaired the spatial resolution due to the energy of the $\beta$ particles emitted by this isotope, and may have been less specific than other studies as a shorter probe was employed and the sections were rather thick $(10 \mu \mathrm{m})$. Most investigators, using ${ }^{35} \mathrm{~S}$ labelled cRNA or cDNA probes have suggested an interstitial localisation (Koury et al. 1988b; Lacombe et al. 1988; Kurtz et al. 1989), with a subset of capillary endothelial cells being considered the most likely candidate (Koury et al. 1988b; Lacombe et al. 1988), although the interstitial fibroblasts have also been proposed (Kurtz et al. 1989).

The level of background in in situ hybridisation studies, and the difficulty in combining this technique with immunostaining have made characterisation of the cell type involved difficult. In all the studies demonstrating an interstitial localisation, cells containing Epo mRNA represent a small proportion of the total number of cells in the kidney. In unstimulated animals a small number of cells in the deep cortex express Epo mRNA. With hypoxia or anaemia, more cells express Epo, at progressively more superficial locations in the renal cortex (Koury et al. 1989). In this quantitative study the number of cells expressing the gene increased exponentially over three orders of magnitude as haematocrit fell from normal to $14 \%$. The majority of positive cells were still in the inner cortex (where they represented about $30 \%$ of all interstitial cells), with few in the superficial cortex or outer medulla (Koury et al. 1989). Another group has found that with severe anaemia induced by haemolysis and irradiation $(\mathrm{Hct}<10 \%)$ most of the interstitial cells in the cortex appeared to contain Epo 
mRNA as did about half of those in the outer medulla (Lacombe et al. 1988). However it is possible that the irradiation (600 rads) considerably altered the interstitial cell population in this study. Previous studies have demonstrated that five days after 1000 rads there is loss of staining for Class II MHC (Ia) and leucocyte common antigen (Hart and Fabre 1981; Gurner et al. 1987). Although this may represent loss of the leucocyte population, the possibility remains that the cells are present but have lost their surface markers (Bohman et al. 1988).

The small number of cells expressing Epo compared to the total population of the kidney has important implications for studying the regulation of the Epo gene at a molecular level. Thus it is very unlikely that nuclear extracts from the whole kidney will be representative of the Epo-expressing cells. Techniques such as DNase1 hypersensitivity to examine chromatin structure or the use of kidney extracts with DNA probes to examine DNA-protein interactions in vitro cannot be applied easily in this situation.

\subsubsection{Relationship of renal Epo expression to tissue oxygenation}

As discussed above, tissue oxygenation is an attractive candidate for the sensed variable underlying regulation of Epo production. In the kidney it is not possible in any straightforward way to correlate the location of Epo-producing cells observed in in situ hybridisation experiments with tissue oxygenation. There are two reasons for this; firstly the difficulty in identifying Epo expressing cells and secondly the complexity of oxygen gradients within the kidney. In fact, in some ways the tissue is a surprising site for oxygensensing in that the blood flow to the kidney is generous compared to its requirement for oxygen, and less than $15 \%$ of the oxygen delivered is extracted. However, in spite of its excellent blood supply and apparently luxurious oxygen delivery, the kidney is very sensitive to conditions of hypoperfusion (Brezis et al. 1984). In accordance with this, it is clear that in regions of the normally functioning renal medulla and cortex the $\mathrm{pO}_{2}$ is very low. The evidence for this, and the possible mechanisms are briefly summarised.

It is clearly established that the vasa recta which supply the renal medulla operate as a counter-current exchanger both for solute and for oxygen. This permits the generation of hypertonicity in the medulla, and provides the basis for efficient extraction of free water from the glomerular filtrate. However, as a consequence levels of oxygenation in the inner medulla are very low (consistently at the level of $10 \mathrm{mmHg}$ in the dog) (Baumgärtl et al. 1972). Certainly in terms of mean oxygenation this is the most hypoxic region of the 
kidney. However, Epo mRNA is not observed in this region in in situ studies so it is reasonable to suppose either that hypoxia is not the sole determinant of Epo expression, or that cells with the capacity for Epo gene expression are not present in the inner medulla.

By contrast to the inner medulla, in the cortex the mean $\mathrm{pO}_{2}$ is substantially higher. However, a marked heterogeneity is observed in $\mathrm{pO}_{2}$ values, whereas in the medulla the $\mathrm{pO}_{2}$ is rather constant. It is also clear that in areas of the cortex the level of oxygenation is much below arterial $\mathrm{pO}_{2}$. In the dog kidney, recordings with platinum microelectrodes (estimated to average $\mathrm{pO}_{2}$ over a region of 3-9 $\mu \mathrm{m}$ ) demonstrated occasional regions of the cortex where the $\mathrm{pO}_{2}$ was 1-2 mmHg (Baumgärtl et al. 1972). There is good evidence for a diffusion shunt operating in the renal cortex. Thus all microelectrode $\mathrm{pO}_{2}$ recordings in the rat cortex were at least $20 \mathrm{mmHg}$ below arterial $\mathrm{pO}_{2}$, and when individual surface glomeruli were examined the $\mathrm{pO}_{2}$ was substantially below that of arterial blood and was increased very little by ventilation with pure oxygen (Schurek et al. 1990). These authors calculated that half of the oxygen in arterial blood had been lost in the preglomerular shunt. It has been suggested that the anatomical site of this shunt is the interlobular vessels, where thin-walled veins run immediately adjacent to arteries for a significant distance (although in vivo the degree of contact is almost certainly less than this due to the presence of a periarterial sheath of connective tissue) (Kriz 1987). In addition there may be similar shunting at the level of the interlobar and arcuate vessels. Not only does this act as a diffusion shunt for oxygen, but also for carbon dioxide, resulting in high levels of $\mathrm{pCO}_{2}(60-70 \mathrm{mmHg})$ in the renal cortex. Since carbon dioxide diffuses an order of magnitude more freely than oxygen, the levels of $\mathrm{CO}_{2}$ recorded show much less variation than those of oxygen. This shunt diffusion also probably leads to an increase in the surface temperature of the kidney, due to trapping of locally produced heat. Since the Böhr effect will result in a right shift of the haemoglobin oxygen-affinity curve, the higher levels of $\mathrm{pCO}_{2}$ and temperature in the cortex may increase the local availability of oxygen.

Actual measurements of $\mathrm{pO}_{2}$ in the cortex of the rat kidney in vivo were below $5 \mathrm{mmHg}$ in $20 \%$ of locations in the cortex, and in $35 \%$ of locations in the medulla (Schurek et al. 1990). In the cortex there is marked heterogeneity in recordings, with well-oxygenated areas immediately adjacent to low values of oxygen tension. With increasing depth in the cortex there is a gradual fall in mean $\mathrm{pO}_{2}$, and then a steep decline in the region of the deep cortex 
and outer medulla. In the cortex very steep local gradients of oxygenation have been recorded (up to $42 \mathrm{mmHg}$ over a distance of $10 \mu \mathrm{m}$ ), although the majority of gradients were $0-3$ $\mathrm{mmHg}$ per $10 \mu \mathrm{m}$.

In response to relatively mild stimulation, Epo-expressing cells (as judged by in situ hybridisation) are, in fact, observed in the deep cortex and to a lesser extent in the outer medulla (Koury et al. 1989). Furthermore, they appear to be located in clusters. It is intriguing that this is the region where mean $\mathrm{pO}_{2}$ is falling rapidly. However, given the complex nature of renal oxygenation, it is hard to relate this distribution directly to local $\mathrm{pO}_{2}$, although it is certainly possible (in view of the considerations above) that the areas where Epo production is activated are relatively hypoxic.

In addition to the evidence for shunting of oxygen in the cortex, other factors may contribute to regional hypoxia. Thus the level of oxygen consumption by metabolically active cells is likely to be important. The majority of energy expenditure by the kidney is related to tubular reabsorption of the glomerular filtrate; an attractive proposal is that the blood flow determines both oxygen supply, and through alterations in GFR also alters the oxygen demand in parallel. This would, perhaps, allow blood oxygen content to be sensed independently from changes in renal blood flow.

Oxygen consumption is predominantly by the proximal convoluted tubule. In animals acetazolamide has been used to decrease proximal tubular work. This reduces the Epo response, whereas other diuretics which act on different parts of the nephron do not (Eckardt et al. 1989a). In the isolated perfused rat kidney Tan attempted to manipulate oxygen consumption independently of oxygen supply. Perfusion with a hyperoncotic solution abolished glomerular filtration and Epo mRNA formation; however, no measurable change in oxygen consumption occurred (Tan 1992). Furthermore, Tan was unable to show an effect of acetazolamide on Epo mRNA levels in the hypoxic isolated perfused kidney. In this context it is interesting that hyperviscosity in humans and experimental animals (due to hypergammaglobulinaemia or dextran) results in inappropriately low levels of Epo production (Singh et al. 1993).

Another important factor in determining regional oxygen delivery may be differences in haematocrit. The haematocrit of blood taken from the kidney is reduced, and it has been proposed that the afferent arterioles, which are generally arranged perpendicularly to the interlobular arteries might preferentially be supplied with plasma rather than red blood cells. Red cell enriched blood might then bypass the tubular circulation via direct arteriolar- 
venular connections; however, no anatomical evidence of such channels has been found. Interestingly, the afferent arterioles of deep glomeruli are orientated at angles greater than $90^{\circ}$ to the direction of blood flow whereas in more superficial glomeruli the angle is less than $90^{\circ}$ (Bankir et al. 1987). This anatomy of the renal arterial circulation probably favours streaming of red blood cells towards the superficial cortex; and indeed injected microspheres are preferentially located in superficial glomeruli. Thus it is possible that the oxygen content of blood delivered to the deep part of the cortex and the medulla is substantially lower than that reaching the superficial cortex.

Furthermore, whereas the majority of the peritubular capillary plexus is supplied by the efferent arteriole of adjacent glomeruli, in the deep cortex efferent arteriolar flow goes to the vasa recta of the medulla and the medullary rays. The outer stripe of the medulla is supplied principally by the wide venous vasa recta ascending from the deeper parts of the medulla. Although this blood is well-oxygenated because it receives oxygen by diffusion from the descending vasa recta this adds further to the difficulties in considering oxygen supply in this region (Bankir et al. 1987).

Local renal oxygenation is not only of interest in the context of Epo formation, but is also important in understanding the pathogenesis of acute renal failure. When renal oxygenation is impaired in animal models of acute renal failure two regions of the nephron appear to be particularly susceptible to morphological damage; the medullary thick ascending limb of Henle's loop and the P3 portion of the proximal tubule in the outer stripe of the outer medulla. Examination of the medulla under these circumstances shows that the damaged structures in each zone are located further from the vascular bundles; this makes it probable that the susceptibility is determined by regional oxygen gradients in the outer medulla (Schurek and Kriz 1985). In isolated perfused kidneys if oxygen delivery was increased by the presence of erythrocytes in the perfusate the necrosis did not occur, further suggesting that the damage was due to hypoxia. However, this region, which appears sensitive to hypoxic damage, does not correlate directly with that in which Epo gene expression is observed in response to anaemia or hypoxia.

Thus at the present time it is uncertain whether the distribution of Epo-expressing cells in the kidney is related primarily to the local level of oxygenation, and these observations can provide no evidence for (or against) the idea that the $\mathrm{pO}_{2}$ in the region of individual Epo producing cells is the sensed variable. As discussed above, detection of blood oxygen 
availability by measuring local $\mathrm{pO}_{2}$ would require the removal of some oxygen. At least two processes could provide this in the kidney; firstly the removal of oxygen by shunt diffusion and secondly local consumption of oxygen.

The basic hypothesis that anaemia is sensed as tissue hypoxia is attractive. However, some experiments with lower animals suggest that, at least in these species, hypoxaemia (which should induce tissue hypoxia) does not produce an erythropoietic response. Thus frogs respond to anaemia with the production of an erythropoietic factor, but no response to hypoxia (or cobalt) could be demonstrated (Rosse et al. 1963). Similarly, box turtles exposed to an altitude of 35,000 feet for 110 days did not develop erythrocytosis (Altland and Parker 1955). Possible explanations are that the appropriate hypoxic stimulus was not applied, or that the sensing system is fundamentally different in these species. However this could indicate the presence of mechanisms for sensing anaemia which do not depend on tissue hypoxia.

In situ hybridisation studies in the kidney have given a further mechanistic insight into regulation of Epo production; it appears that the level of Epo mRNA is closely correlated with the number of cells expressing the gene, and that the level of Epo mRNA per cell is fairly constant (Koury et al. 1989). This observation led to the suggestion that individual cells are recruited in an all-or-none fashion. How this may relate to local $\mathrm{pO}_{2}$ is unknown.

\subsubsection{Epo expression in the adult liver}

Although the kidney is the major source of Epo production in the adult, there is also significant Epo production by the liver. Thus anephric humans have been shown to have detectable levels of circulating Epo, and these levels can rise sharply in response to a fall in haemoglobin (Naets and Wittek 1968; Caro et al. 1979; Cotes 1982). Nephrectomised animals still have measurable levels of Epo, which increase in response to hypoxia, anaemia or cobalt (Fried and Kilbridge 1969; Schooley and Mahlmann 1972a). Hepatectomy in these animals has been shown to abolish this rise, leading to the conclusion that the liver is the major source of extra-renal Epo (Fried 1972). This has been confirmed by mRNA analysis. Using Northern analysis Bondurant and Koury were able to demonstrate Epo mRNA in the liver of anaemic mice, and estimated that this amounted to about 12 to 15\% (Bondurant and Koury 1986) of that produced by the kidney. In anaemic or hypoxic adult rats the liver was shown to produce up to $33 \%$ of total Epo mRNA, with the proportion being lower with less severe anaemia or hypoxia (Tan et al. 1992). This proportion was higher than the Epo levels observed in earlier studies involving bilateral nephrectomy, which estimated 10 to $18 \%$ (increasing 
with more severe stimulation) as the extrarenal contribution to Epo production in the rat (Erslev et al. 1980). One possible reason for this difference is that bilateral nephrectomy itself reduces the level of hepatic Epo mRNA for a given stimulus (Eckardt et al. 1992b). In support of this the amount of Epo produced by nephrectomised rats decreased progressively with time after nephrectomy (Schooley and Mahlmann 1972a).

An important issue is why liver Epo production cannot substitute adequately for renal production in renal disease. This is not fully understood. As discussed above, there is no doubt from human and animal observations that anephric individuals have the ability to produce Epo. One potential explanation is that the liver response is only sufficient to maintain adequate erythropoiesis in response to more severe stimuli. Some experimental evidence for this comes from studies of the rat (Tan et al. 1992). To what extent this might relate to different patterns of local oxygenation in the two organs, and different cellular or molecular mechanisms remains unknown. Other mechanisms may be important; thus it is plausible that hepatic Epo mRNA is less efficiently translated, the hormone is not released into the circulation or is more rapidly removed by the liver than when it is derived from the kidney.

\subsubsection{Epo production by the regenerating liver}

Unlike most other mammalian organs, the liver has a marked ability for regeneration, and there is convincing experimental evidence that the regenerating liver (following sub-total hepatectomy) has a very substantially enhanced ability to synthesise Epo in response to hypoxia (Naughton et al. 1977). To what extent these observations concerning regenerating liver are due to the altered pattern of blood supply in these circumstances is not known. Interestingly in humans liver damage, either viral or toxic in nature, in patients with renal failure produced a significant rise in haemoglobin levels, starting some weeks after the insult, and hence probably being associated with liver regeneration (Simon et al. 1980).

\subsubsection{Cellular site of Epo production in the liver}

Considerable evidence from cell separation studies (Lacombe et al. 1991; Schuster et al. 1992; Eckardt et al. 1993) and in situ hybridisation suggests that the Epo gene is expressed by two populations in the liver, one of which are the hepatocytes (Koury et al. 1991; Schuster et al. 1992). Estimates of the relative importance of these two cell populations have been variable. In neonatal rats exposed to carbon monoxide positive cells by in situ hybridisation were not parenchymal (Kurtz et al. 1989). In anaemic mice poly A-selected RNA from parenchymal and non-parenchymal fractions contained similar amounts of Epo mRNA (Lacombe et al. 1991). Using in situ hybridisation, in anaemic mouse liver $80 \%$ of the cells 
containing Epo mRNA appeared to be hepatocytes (Koury et al. 1991). A similar proportion was observed in anaemic-hypoxic rats (Schuster et al. 1992). In Koury's study forty-fold more cells were positive in severely anemic mice than in controls; however positive cells were still infrequent and the level of signal per cell was lower than that seen in the kidney. It was not possible to assess the distribution of Epo mRNA containing cells in the liver lobule, nor to assess whether cells were recruited in an all-or-none fashion as suggested in the kidney.

The non-parenchymal cell population has not been characterised, although it has been described as having small dense nuclei and little cytoplasm. There is some evidence that Kupffer cells isolated from regenerating rat liver can produce Epo in culture; raising the possibility that these are the non-parenchymal cell type identified by in situ hybridisation (Paul et al. 1984).

\subsubsection{Epo production in the foetus and neonate}

Bilateral foetal nephrectomy followed by induction of anaemia was used to demonstrate that in the foetal goat the kidney was not the source of Epo (Zanjani et al. 1974). Subsequently organ removal studies showed clearly that the foetal liver is the major site of foetal Epo synthesis. Moreover, Epo was found in perfusate from isolated liver, but not kidney, of anaemic sheep foetuses (Zanjani et al. 1977).

The developmental switch from hepatic to renal Epo production is timed rather differently in different species, and the mechanism is not clearly understood. In the sheep, organ removal experiments at various ages indicated that the kidney becomes a significant site of Epo production (about $30 \%$ of the total, measured by bioassay) in response to anaemia in late gestation, gradually increases in importance, and is the dominant site at 40 days post partum (Zanjani et al. 1981). In the rat the switch to renal production occurs predominantly postnatally, and in the neonatal period the liver is still the dominant site of Epo synthesis. Thus in the unstimulated, hypoxic or carbon-monoxide treated neonatal or juvenile rat the liver contains substantially more Epo mRNA than the kidney (Eckardt et al. 1992b). In contrast, in the mouse the kidney is the dominant site of Epo mRNA in late gestation and throughout postnatal life (Koury et al. 1988a)

One possibility for the mechanism underlying this developmental switch would be that it was related to the circulatory changes that occur at parturition, since the foetal liver recieves blood via the ductus venosus, which comes from the site of oxygenation in the placenta 
(although this blood is partially channelled to the heart, bypassing the liver). This seems unlikely, since the pattern of the transition is so variable from one species to another. A contributory factor might be the transition to adult-type, lower affinity haemoglobin for oxygen carriage after birth, which will significantly alter oxygen availability.

Interestingly, in sheep in response to maternal anaemia a rise in circulating foetal Epo occurs some hours before a rise in the maternal circulation is detectable (Zanjani et al. 1974). It is possible that this is due to the higher affinity of foetal haemoglobin for oxygen, leading to lower oxygen availability in the foetus.

\subsubsection{Epo production from organs other than kidney and liver}

\section{Normal tissues}

In the rat, in addition to the liver and kidney, the spleen and lung have been shown to contain Epo mRNA by RNase protection and competitive PCR (Tan et al. 1991; Fandrey and Bunn 1993); however the amounts detected were very low compared to those seen in the liver and kidney, and their significance is uncertain. An important point is that Epo mRNA was detected in the spleen of hypoxic animals (Tan et al. 1991) and lung of anaemic animals (Fandrey and Bunn 1993) but was not detected in the same organ of control animals in each study. Interestingly, although Tan et al. also found low levels of Epo mRNA in the lung this was not increased in hypoxic animals (Tan et al. 1991).

The testis and brain have also been shown to contain Epo mRNA in rats; in unstimulated animals this was quantitatively significant when compared with renal expression. Thus in normoxic rats the quantity of Epo mRNA in the testes and brain was $30 \%$ and $5 \%$ (respectively) of the low levels in the kidney (Tan et al. 1992). The level of Epo mRNA in both organs rose in response to severe hypoxia, but only by about three-fold. Since the level in kidney and liver was induced 200-fold, the contribution to total Epo mRNA under these circumstances was quantitatively insignificant (Tan et al. 1992).

The significance of production of Epo in these sites is uncertain. Thus it is not known whether this Epo mRNA can be translated, and both the testis and brain have a barrier to diffusion between the parenchymal tissue and blood, thus it is not clear that Epo formed in these tissues would necessarily reach the bloodstream. An important point raised by the detection of hypoxically-modulated Epo expression in these organs is that it suggests that at least some elements of the oxygen-sensing mechanism are present in sites other than the kidney and liver. 
Some evidence has been put forward for two other sites of Epo synthesis. Firstly, bone marrow macrophages have been thought to produce Epo on the basis of in situ hybridisation and cell isolation techniques (Rich et al. 1988). However, Epo mRNA has not been detected in the bone marrow either by RT-PCR or RNase protection. Rodent salivary glands were thought to contain high levels of Epo in radioimmunoassays (Clemons et al. 1987); again, no Epo mRNA was detected by RNase protection. It is likely that the reason for the apparent detection of Epo in the salivary gland by RIA may be attributable to the presence of substances in this tissue which are capable of degrading the radiolabelled tracer in the assay (Tam et al. 1989).

\section{Malignant tissues}

There is clear evidence that Epo can be produced in a number of pathological situations. Several tumors are associated with polycythaemia; in particular, renal cell carcinoma, nephroblastoma, hepatoma, cerebellar haemangioblastoma and uterine fibroids. This has been studied in most detail in three patients with renal cell carcinoma who were shown to have elevated levels of immunoreactive serum Epo. Epo mRNA was demonstrated in the epithelial tumor cells by in situ hybridisation. No DNA rearrangement was observed in the tumors (Da Silva et al. 1990). Epo has also been demonstrated in the cyst fluid and Epo mRNA in tumor tissue in a cerebellar haemangioblastoma (Trimble et al. 1991). A cell line established from a human renal cell carcinoma has been shown to produce Epo stably in tissue culture (Sherwood and Shouval 1986). This expression in renal epithelial cells is in contrast to the interstitial expression of Epo mRNA in the majority of in situ hybridisation studies performed on normal renal tissue. That epithelial cells from the kidney can produce Epo in the context of malignant transformation is interesting in the context of Epo gene expression by liver parenchymal cells (i.e. hepatocytes).

The cells giving rise to these tumours are not known to produce Epo. One theoretical possibility would be that Epo production in these tumors relates to hypoxic exposure of cell types which are normally well oxygenated. Alternatively, malignant transformation is often associated with abnormal expression of a range of genes.

\subsubsection{Epo production in renal disease}

The majority of patients with renal failure are anaemic. The anaemia of renal disease is probably multifactorial; however, the dominant cause appears to be a relative deficiency of Epo production (Caro et al. 1979; Cotes et al. 1989a; Eschbach 1989). Thus patients with renal disease have measurable levels of Epo, often within the normal range for patients 
with normal haematocrits, but which are one to two orders of magnitude lower than patients with equivalent anaemia and normal kidneys (Cotes 1982). The reason for the relative deficiency of Epo observed in human renal disease is not known. Since the cells responsible for Epo production have not been identified, it is not known whether they are present in diseased kidneys or not. It is well-recognised that bilateral nephrectomy in dialysed patients impairs erythropoiesis (Kominami et al. 1971), suggesting that renal synthesis of the hormone remains important. Moreover, it is known that uraemic patients can increase Epo levels when exposed to altitude (Blumberg et al. 1973) or acute hypoxic stress due to intercurrent illness (Chandra et al. 1988), although in these instances it was not posssible to determine whether the source was the kidneys, liver or even another tissue. An anephric patient described by Cotes showed an increase in Epo level from $<5$ to $99 \mathrm{mIU} / \mathrm{ml}$ when the haemoglobin dropped from 5.0 to $3.5 \mathrm{~g} / \mathrm{dl}$ (Cotes 1982).

The question of the reserve for Epo synthesis in uraemia has been investigated in rats (Tan et al. 1991). Animals were studied four weeks after five/sixth nephrectomy when they had developed anaemia and uraemia. Although they had reduced renal Epo mRNA, in response to hypoxia they were able to increase this level fifty fold; moreover the level of hepatic Epo mRNA in response to severe hypoxia was not different from that of control animals. Thus at least in this model there was considerable hepatic and renal potential for Epo synthesis.

\section{Autosomal dominant polycystic kidney disease}

Patients with autosomal dominant polycystic kidney disease and renal failure tend to be less anaemic than those with renal failure due to other causes. Eckardt and colleagues have shown very high levels of Epo in some cysts (up to $3.2 \mathrm{U} / \mathrm{ml}$ ); particularly in small cysts $(<0.5 \mathrm{ml})$ with high sodium concentrations $(>100 \mathrm{mmol} / \mathrm{l})$. Using in situ hybridisation they demonstrated Epo mRNA in stromal cells located in the cyst wall (Eckardt et al. 1989b). The high sodium concentration suggested that the cysts containing Epo were of proximal tubular type. Interestingly there was no correlation between the oxygen tension or $\mathrm{pH}$ and the Epo level in fluid from individual cysts. The inverse relationship of haematocrit to serum Epo level seen in normal populations did not appear to apply to patients with ADPKD; in fact a positive correlation may apply, suggesting that in this condition there may be autonomous production of Epo. 
It is intriguing that patients with acquired cystic disease on renal replacement therapy have higher levels of Epo than patients without cysts, and a tendency to higher levels of haemoglobin. This suggests that the effect on Epo production is related to the presence of cysts in the kidney, rather than to the underlying cause of the cysts (Edmunds et al. 1991).

\subsection{REGULATION OF THE EPO GENE - MOLECULAR BIOLOGY}

The two most striking characteristics of Epo gene expression are the tissue specificity of Epo gene expression and the high level induction of gene expression by anaemia and hypoxia. Considerable information concerning both of these has been obtained from studies using transgenic mice. In these experiments cloned DNA from the human Epo locus was injected into mouse zygotes (see below). In such experiments the human DNA integrates into the mouse genome, resulting in transgenic mice. The pattern of expression of the transgene in the mice will depend on cis-acting elements included in the injected DNA. The site of integration of the DNA into the mouse genome often influences the level of expression of transgenes; this indicates that not all the cis-acting elements involved in controlling gene expression are present in the transgene. The extent of DNA required for completely faithful expression will depend on the location of the cis-acting elements. It is clear is that such elements can be located at long distances from the gene they control (e.g. $60 \mathrm{~kb}$ in the case of the human $\beta$ globin gene; Crossley and Orkin, 1993). Experiments with human Epo transgenes (using up to $22 \mathrm{~kb}$ of DNA) indicate the position of cis-acting elements involved in tissue-specificity and induction of gene expression. These transgenic experiments did not produce completely faithful expression of the transgene, indicating that important cisacting sequences are located outside the $22 \mathrm{~kb}$ used in these experiments.

The second experimental method used to study Epo gene expression has involved the use of tissue culture cells. As discussed above, in studies of intact animals or isolated kidneys no correlation of local $\mathrm{pO}_{2}$ with Epo gene expression has been attempted. The development of a suitable tissue culture model has therefore been crucial to further understanding of the hypoxic induction of Epo gene expression.

\subsubsection{Mechanisms of tissue specificity: studies using transgenic mice}

Studies of human Epo transgenes in mice by Semenza and colleagues have given valuable information about the location of sequence governing tissue specificity. The transgenes studied included different amounts of human flanking sequence and the results of each transgenic experiment are summarised below and illustrated schematically in Figure 1.2. 


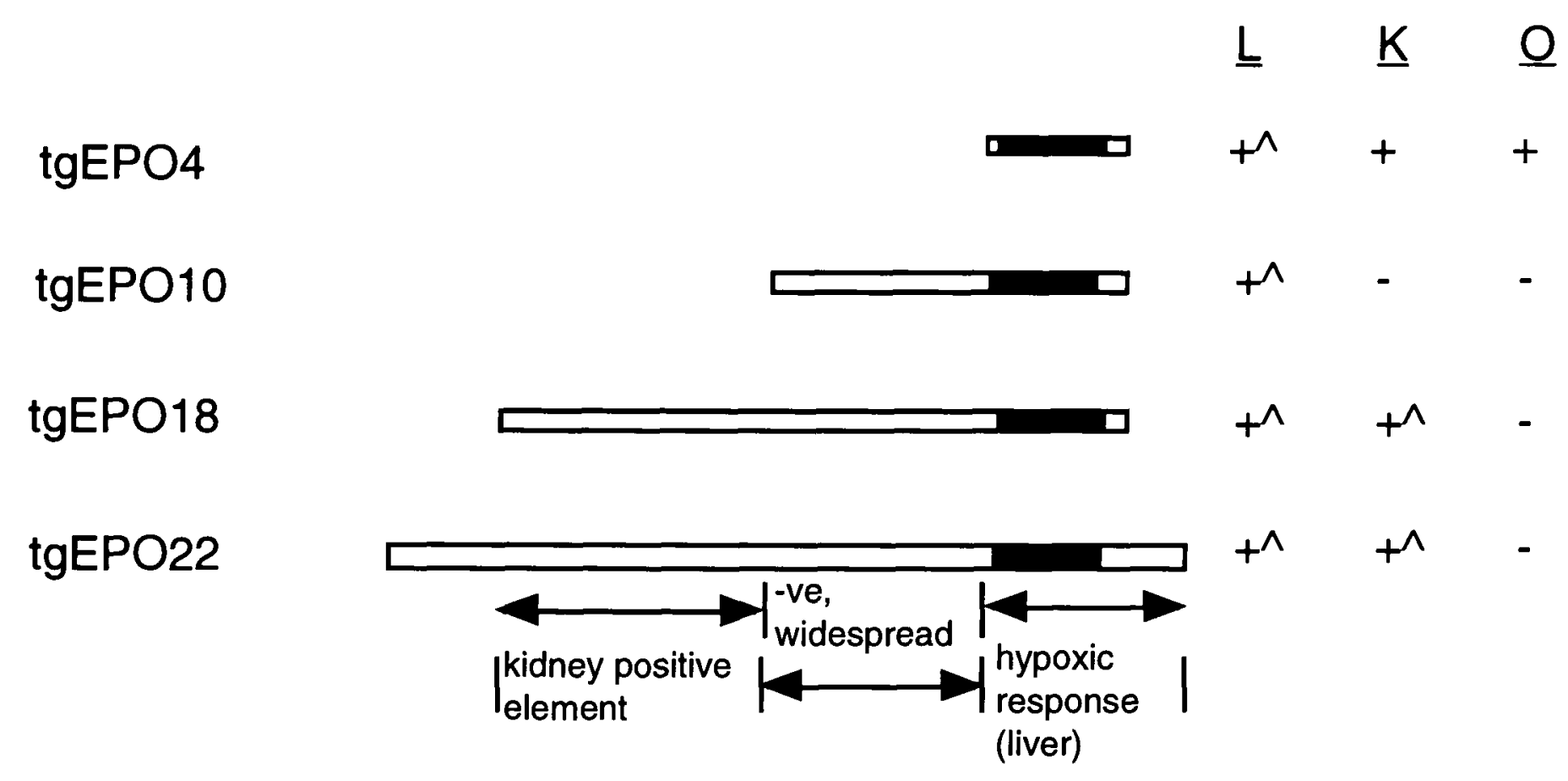

Figure 1.2 - Summary of Semenza's transgenic experiments. In each case the name of the transgene indicates the length of sequence (eg tgEPO4 was 4kb). The human Epo gene is solid. Flanking sequence is open. $L=$ liver, $K=$ kidney, $O=$ other tissues. $+=$ expression detected, $\wedge^{\wedge}=$ inducible expression. Beneath the constructs the conclusions drawn about the location of cis-acting elements are summarised.

Three out of four transgenic lines expressing a human Epo transgene with $0.4 \mathrm{~kb} 5$ ' flanking sequence and $0.7 \mathrm{~kb} \mathrm{3'}$ flanking sequence (tgEPO4, which included the $3^{\prime}$ enhancer region discussed below) were polycythaemic (Semenza et al. 1989; Semenza et al. 1990). One line did not express the transgene. Transgene mRNA was found in the liver of all four lines which did express the transgene, but also at a high level in the three polycythaemic lines in other tissues (kidney, brain, spleen, heart, lung and thymus in a variable pattern). The transgene mRNA in liver was of the predicted $(1.6 \mathrm{~kb})$ length, but was longer in other tissues. Anaemia induced higher level expression in the liver in each line, and one line was also shown to respond to cobalt. Expression in other tissues, in particular kidney, was not induced by anaemia. This suggested that the construct used contained anaemia-responsive elements (at least capable of operating in liver) and that important negative cis-acting sequence lay more distant from the gene. Moreover, oxygen-regulation in the kidney appeared to depend on more distal elements.

Transgenes with an additional $5.6 \mathrm{~kb}$ of 5 ' sequence (tgEPO10) showed inducible expression in the liver, but were not expressed in other tissues, suggesting that an element lying between 0.4 and $6.0 \mathrm{~kb} 5$ ' to the human gene was involved in suppressing expression in these tissues (Semenza et al. 1990). In this experiment nine transgenic lines were established, of which five were shown to express the transgene. In four of these lines, expression was confined to the liver. The fifth line showed aberrant expression, and the liver expression was not inducible. Interestingly three further lines, where transgene expression was not detected in any tissue (even in response to anaemia), were polycythaemic. This suggests either that 
other tissues were expressing the transgene or that the detection method used (Northern blotting of total mRNA) was insufficiently sensitive to detect physiologically important expression.

Two further transgenes have been studied (Semenza et al. 1991a); one with $14 \mathrm{~kb}$ of $5^{\prime}$ sequence and $0.7 \mathrm{~kb}$ of $3^{\prime}$ sequence (tgEPO18), the other with $16.5 \mathrm{~kb}$ of 5 ' sequence and $2.2 \mathrm{~kb}$ of 3 ' sequence (tgEPO22). Both of these showed regulated expression in liver and kidney as follows. Seven different transgenics (six lines and one founder) expressed the tgEPO18 transgene; they were all polycythaemic. The level of human Epo mRNA in the kidney was similar to that of the mouse Epo gene while the level in the liver was greater than that of the endogenous gene, and was inducible in both organs. Transgene expression was not observed in total mRNA from other tissues. Four transgenic lines expressed the $\operatorname{tgEPO} 22$ transgene; three of these were polycythaemic. The expression pattern of $\operatorname{tgEPO} 22$ was essentially the same as that of tgEPO18. $\operatorname{tgEPO} 22$ was additionally shown to express in an inducible fashion in foetal liver at 14 days post coitum. In the kidney, in situ hybridisation was used to localise mouse and human Epo mRNA; signals were found in similar numbers and types of cells. Thus it appears from the results of the tgEPO18 and $\operatorname{tgEPO} 22$ transgenes that in the human an important element for renal expression lies between 6.0 and $15 \mathrm{~kb} 5$ to the gene, and that an element important for suppression of liver expression lies outside the sequence employed in the largest transgene. An alternative explanation is that the mouse trans-acting factors responsible for repression of liver expression are sufficiently divergent from those in the human that they cannot exert this effect on putative human cis-acting sequence contained in the transgenes.

Interestingly in mice over-expressing the human Epo gene in the liver (tgEPO10), the expression as detected by in situ hybridisation was restricted to hepatocytes, and was not observed in the non-parenchymal cell population. Unlike in situ studies of the mouse Epo gene in the kidney, greater stimulation resulted in increased levels of Epo mRNA per hepatocyte, as measured by counting silver grains. The hepatocytes expressing the human Epo gene were clearly in a pericentral distribution (Koury et al. 1991). This is an important observation, since surface measurements of oxygen levels in the liver have clearly demonstrated that this is the most hypoxic area of the liver lobule (Jungermann and Katz 1989). However, it is an important caveat that these findings related to an aberrantly expressed human transgene; the number of cells containing mouse Epo mRNA was too low for 
assessment of either their distribution, or the amount of mRNA per cell. In fact, in a study of neonatal rats exposed to carbon monoxide cells positive for Epo mRNA were found in periportal regions and randomly distributed between parenchymal cells (Kurtz et al. 1989).

\subsubsection{Cell culture models of Epo production}

Two central issues could only be examined in a restricted fashion in the animal experiments described above. Firstly, in whole organs it has been very difficult to correlate $\mathrm{pO}_{2}$ in the region of a particular cell with Epo expression. Secondly, the Epo-producing cells form only a tiny fraction of the cells in the kidney; thus studies of the whole organ are of limited utility in understanding the intracellular processes governing gene expression. Much of the current knowledge concerning cellular and molecular mechanisms modulating Epo production has therefore been derived from cell culture experiments, in which a homogenous population can be defined and oxygenation can be controlled.

To date, Epo producing cells have not been isolated from the kidney in spite of extensive screening of cell lines in tissue culture. Although several cell lines and heterogenous culture systems were shown to produce erythropoietic activity (although in some instances this was only demonstrable in in vitro bioassays), this was usually not modulated by hypoxia, was at a low level or was not stable with time. For instance, primary mesangial cell cultures from rats were found to produce an erythropoietic substance as measured in in vitro bioassay, and formation was increased several fold in response to cobalt $(10 \mu \mathrm{M})$ or hypoxia $(1 \%$ oxygen). However, the concentrated culture medium was not active in polycythaemic mice, the level of Epo was only estimated at $15 \mathrm{mIU} / \mathrm{ml}$ (Kurtz et al. 1983) and apparently does not compete with Epo in immunological assays (Jelkmann 1992).

An exception is the human renal carcinoma cell line RC-1, described by Sherwood; preliminary experiments with this cell line having shown a response to hypoxia (3\% oxygen) and very high concentrations of cobalt $(10 \mathrm{mM})$. Under $3 \%$ oxygen the levels of Epo in the culture medium were greater than $1000 \mathrm{mIU} / \mathrm{ml}$ (Sherwood and Shouval 1986). However, these cells have not been widely available and the response to hypoxia was of low magnitude.

\section{Hepatoma cells produce Epo in a regulated fashion}

In 1987 Goldberg and colleagues demonstrated that the human hepatoma cells HepG2 and Hep3B produce Epo at a high level in tissue culture. Furthermore, Epo production was increased under hypoxic conditions (Goldberg et al. 1987). This provided a model system for the study of Epo gene regulation. These investigators explored a range of conditions; 
they found $1 \%$ oxygen to be a better stimulus than $2 \%, 3 \%$ or $5 \%$ oxygen. They were able to show a 3 fold increase in the rate at which Hep G2 cells released Epo into culture medium, and an 18 fold increase in Hep 3B cells. A correlation was observed between the level of Epo mRNA in the cells and the rate of release of Epo into the culture medium. Over 24 hours near-confluent cultures formed approximately $250 \mathrm{mIU} / \mathrm{ml}$ of Epo. One important conclusion from these experiments is that hypoxic-sensing and Epo production can occur in the same cell (although it remains possible that this is not the situation in the kidney).

Although these hepatoma cells display several characteristics of well-differentiated liver parenchymal cells (e.g. synthesis of plasma proteins), it is necessary to be cautious about drawing parallels between normal hepatocytes and tumor cells. However, several lines of evidence suggest that hepatoma cells do provide a suitable model system for Epo gene expression in the kidney and liver.

\section{Smilarities of physiological regulation of Epo production and that observed in hepatoma cells}

Although it is straightforward to study the relationship between ambient $\mathrm{pO}_{2}$ in a tissue culture incubator and Epo production by hepatoma cells, it is very difficult to relate this to the tissue $\mathrm{pO}_{2}$ in the functioning liver and kidney. This is for two reasons; firstly, the local $\mathrm{pO}_{2}$ in tissues has not been correlated with Epo gene expression in animal studies. Second, although the incubator $\mathrm{pO}_{2}$ can be closely controlled the $\mathrm{pO}_{2}$ in the region of the tissue culture cells themselves is much less certain. Interestingly, the constitutive production of Epo in these cell cultures appears to be increased at higher cell density, with less inducibility. An attractive, but unproven explanation is that the sensing-mechanism in hepatoma cells is influenced by local oxygen consumption in addition to the ambient level of oxygen in the incubator.

It has, however, been possible to show that hepatocytes isolated from the liver of juvenile hypoxic rats are capable of expressing increased Epo mRNA in vitro in response to hypoxia (15 fold effect of $3 \%$ compared to $21 \%$ oxygen) or cobalt (a small effect) although the ability to express the Epo gene is lost quite rapidly in vitro (Eckardt et al. 1993). This provides important evidence that freshly isolated hepatocytes behave in a similar way to hepatoma cells.

Much of the evidence that hepatoma cells provide a good model of physiological regulation of the Epo gene is derived from similar pharmacological responses in hepatoma cells, isolated kidneys and whole animals. Cobalt has long been known to increase erythropoiesis in 
rodents and humans, by increasing the levels of Epo. The demonstration that cobaltous chloride in the tissue culture medium induced Epo gene expression by hepatoma cells in a dose-dependent fashion was therefore an important parallel with physiological regulation of the gene in vivo (Goldberg et al 1988). As discussed below, an attractive explanation for the mechanism of action of cobalt is that it is acting through substitution in a haem protein.

Since the stimulus to Epo production appears to be tissue oxygenation, one possible underlying mechanism was that oxygen-sensing at a cellular level was dependent on compromising cellular respiration. This has been investigated in vivo using the following; potassium cyanide, which prevents electron transport to oxygen by blocking cytochrome aa3; fluoroacetate, which inhibits aconitase, an enzyme in the citric acid cycle; and monoiodoacetic acid which inhibits glycolysis. Administration of cyanide, fluoroacetate, monoiodoacetic acid or monoiodoacetic acid combined with either of the other agents to rabbits and rats did not induce Epo synthesis (assessed by in vivo bioassay), even at the highest doses compatible with survival. Furthermore, administration of these agents did not impair the hypoxic induction of Epo (Necas and Thorling 1972; Necas and Neuwirt 1972). Similarly, neither cyanide nor other inhibitors of oxidative phosphorylation induced Epo mRNA in isolated perfused non-filtering rat kidneys, or prevented induction by hypoxia (Tan and Ratcliffe 1991). Thus cyanide and other inhibitors of cellular metabolism do not appear to alter Epo production, although they would be expected to mimic many of the metabolic consequences of hypoxia at a cellular level. This makes it unlikely that compromise of cellular respiration is involved in the sensing mechanism. That cyanide does not induce an Epo response in hepatoma cells provides further evidence for use of these cells as a model system for studying Epo gene expression (Goldberg et al. 1988).

\section{Evidence for involvement of a haem protein}

A series of experimental observations using Hep3B cells have led Goldberg and colleagues to suggest that the hypoxia-response system involves a rapidly turning over haem protein (Goldberg et al. 1988), which would constitute a specific molecular oxygen sensor. The evidence for this was as follows. Cycloheximide, an inhibitor of protein synthesis, was shown to abolish the Epo mRNA response to hypoxia suggesting that synthesis of protein was a necessary step in the response. Certain transition metals (cobalt, nickel and manganese) could induce Epo mRNA, and it was suggested that this was due to substitution for iron in newly-formed haem molecules, which would then be stable in a de-oxy conformation. Carbon monoxide, which is a highly selective ligand for the iron atom in reduced haem 
molecules, inhibited the hypoxic response; it had little effect on the response to cobalt, consistent with a failure to ligate cobalt protoporphyrin. Furthermore, preincubation with desferrioxamine (a potent iron chelator) or 4,6-dioxoheptanoic acid (an inhibitor of aminolevulinate dehydratase) reduced the magnitude of the hypoxic response. Subsequently, however, it has been shown that desferrioxamine itself increases the formation of Epo mRNA (Wang and Semenza 1993b), placing some doubt on the precise interpretation of the latter finding. Since these authors showed that desferrioxamine induced Epo gene expression when administered to mice this provides further evidence that hepatoma cells are a valid model for studying Epo gene regulation.

Although there is suggestive evidence for the involvement of a haem protein in the sensing mechanism, such a protein has not been isolated nor has its existence been proven. There is an important precedent for such a system in the soil bacterium rhizobium meliloti which has a well-characterised oxygen-responsive transcriptional control cascade, based on an oxygensensing haem protein (Gilles-Gonzalez et al. 1991; Monson et al. 1992; Agron et al. 1993).

\subsubsection{Definition of hypoxia-responsive cis-acting elements}

In order to understand the mechanism of increased transcription in response to hypoxia one aim is to identify cis-acting elements from the Epo locus which are responsible for transmitting the regulatory effect. Several investigators have used this approach to identify such elements in human and murine DNA by using transient transfection assays.

\section{Enhancer element lying 3 ' to the gene}

Three groups working independently identified a powerful enhancer region lying $3^{\prime}$ to the Epo gene. Beck and colleagues defined a $150 \mathrm{bp}$ segment of DNA lying $120 \mathrm{bp} 3^{\prime}$ to the polyadenylation site of the human Epo gene (Beck et al. 1991).

Semenza analysed his tgEPO10 human transgene (which was inducibly expressed in the mouse liver) for DNase 1 hypersensitive sites. Two sites lying 3 ' to the gene were identified (Semenza et al. 1991b). Functional studies demonstrated that $256 \mathrm{bp}$ from this region could act as a hypoxically-regulated enhancer when transiently transfected into Hep3B cells. Probes from this region were then used in mobility shift assays with nuclear extracts from kidney and liver. Although the results were complex some of the retarded species were induced by hypoxia. 
Pugh et al. identified a $70 \mathrm{bp}$ region $120 \mathrm{bp} 3$ ' to the polyadenylation site of the mouse Epo gene which acted as a hypoxically-regulated enhancer element in the context of human $\alpha$ globin or mouse Epo promoters (Pugh et al. 1991). This element also conveyed responsiveness to cobalt, but not to cyanide, supporting a physiological relevance for this regulatory system.

The area studied by all three groups is highly homologous between mouse and man. The sequence is given in Appendix 1. Further studies have demonstrated that only $24 \mathrm{bp}$ of the human enhancer (Madan and Curtin 1993) or 26 bp of the mouse gene are necessary to convey hypoxically-regulated activity. An important finding is that an inducible DNA binding activity (termed HIF-1) has been demonstrated in bandshift assays (Semenza and Wang 1992). This binding activity is dependent on the first 18 bases of the 3 ' enhancer.

This hypoxia-responsive enhancer element was selected for the experiments described in Chapter 3. This appears to be the most important element for hypoxic induction of Epo gene expression in hepatoma cells. However, there is evidence that other sequences from the Epo locus can convey a hypoxic response, at least under some circumstances.

\section{Enhancer element in the 3' UTR}

Imagawa and colleagues examined two regions; a $1.2 \mathrm{~kb} 5^{\prime}$ fragment extending from 378 bp $5^{\prime}$ to the cap site through the first intron (with a mutated ATG) and a 255 bp region including the $3^{\prime}$ untranslated region (but not including the $3^{\prime}$ enhancer discussed above). These were inserted $5^{\prime}$ and 3 ' to human growth hormone as a reporter gene, and examined both in transient and stable Hep 3B transfectants (Imagawa et al. 1991). In transient transfectants, the 5 ' fragment appeared to confer low level inducibility by hypoxia or cobalt ( 1.7 fold) which was increased to about 2.3 fold if the 3 ' fragment was also present (regardless of orientation). In two stably transfected clones containing both Epo fragments, a much higher level of inducibility was observed (up to 16 fold in response to hypoxia).

\section{Promoter function and the $5^{\prime}$ region of the gene}

Some information about the (rather weak) promoter of the Epo gene has been obtained from in vitro transcription studies. Hypoxic Hep 3B nuclear extracts produced correctly initiated transcripts from an Epo plasmid template (containing $3.4 \mathrm{~kb}$ of Epo sequence, including $0.4 \mathrm{~kb}$ of $5^{\prime}$ flanking sequence and $0.6 \mathrm{~kb}$ of $3^{\prime}$ flanking sequence). Transcription was several-fold less efficient with normoxic extracts, suggesting a hypoxically-inducible promoter was included in the construct (Costa-Giomi et al. 1990). Using a 400 bp fragment 
which covered the same 5' flanking sequence and the cap site, no transcripts of the correct length were detected; in contrast when only $200 \mathrm{bp}$ immediately $5^{\prime}$ to, and including, the cap site was used as the template, transcription was observed with nuclear extract from anaemic (but not control) mouse kidney (Tsuchiya et al. 1992). This implies that this fragment of the 5' flanking region contains an effective promoter for Epo gene expression with element(s) that can confer a hypoxic response. Subsequently these investigators have demonstrated DNA binding activity to the region -40 to -20 bp from the cap site in mobility shift assays with nuclear extracts from kidney and liver of mice exposed to anemia or cobalt, which is not present in extracts from normal mice (Tsuchiya et al. 1993). Another group (Blanchard et al. 1992) has performed functional analyses of the human Epo promoter using transient transfection and a luciferase reporter system; they identified a minimal basal promoter consisting of $91 \mathrm{bp}$ lying immediately $5^{\prime}$ to the transcription start site. Although this promoter was inducible by 2.5 fold under combined stimulation by cobalt and hypoxia, the inclusion of a further $26 \mathrm{bp} 5$ resulted in a 6 to 10 fold induction of reporter activity. Sequence included in this construct was homologous to an area in the $3^{\prime}$ enhancer and was shown to cross-compete in mobility shift assays.

\subsubsection{Post-transcriptional control}

The relative contributions of transcriptional and post-transcriptional regulation to the hypoxic accumulation of Epo mRNA has been studied in hepatoma cells. In response to hypoxia or cobalt a fifty-fold increase in steady state levels of Epo mRNA was observed; however only a ten-fold increase was observed in response to the same stimuli using nuclear run-off (Goldberg et al. 1991). This suggested an additional effect on mRNA stability. Actinomycin D chase experiments to investigate mRNA stability in response to hypoxia were difficult to interpret, since actinomycin D itself greatly increased the stability of Epo mRNA (from a maximum estimate of 1.5 hours to 8 hours). Cycloheximide also increased the half-life of Epo mRNA, leading the authors to suggest that degradation was enhanced in the presence of a rapidly turning over protein (possibly a ribonuclease). Subsequently these authors have demonstrated a binding activity in hepatoma cell extracts using $120 \mathrm{bp}$ from the 3'UTR in electrophoretic mobility shift assays. This activity was not hypoxically regulated in extracts from hepatoma cells, mouse kidney or mouse liver; however it was increased by hypoxia in mouse brain and spleen (Rondon et al. 1991). Although the DNA sequence in this region is conserved between mouse and man, suggesting functional importance, it is currently difficult to assess its role in regulation of the Epo gene. 


\subsection{MAJOR FEATURES OF EPO REGULATION}

The preceeding two sections summarise a substantial body of work concerned with the regulation of Epo production. Although current understanding of the regulation of Epo gene expression is incomplete, there are distinctive features which characterise these responses.

\subsubsection{High amplitude transcriptional response to anaemia or hypoxia}

The stimulus to this is probably a change in $\mathrm{pO}_{2}$ in the region of the cell; there is good evidence that it is not due to a general compromise of cellular metabolism. The increase in mRNA can be induced by cobalt but not by cyanide, and is blocked by cycloheximide. A cis-acting sequence, the 3 ' enhancer, has been defined functionally in hepatoma cells and is associated with a hypersensitive site in human transgenes in mouse liver. More recently a hypoxically-regulated DNA binding activity (termed HIF-1) has been shown to bind to probes from this region.

\subsubsection{Tissue-specific expression}

The gene is expressed in the renal interstitium of the adult, in an as yet unidentified celltype, and to a lesser extent in the liver. The mechanism of transducing changes in blood oxygen availability to increased transcription of the Epo gene are not understood. The cisacting sequences mediating the tissue-specificity of expression have not been defined.

\subsection{MECHANISMS OF TRANSCRIPTIONAL CONTROL}

The principal areas of study in this thesis have been the tissue-specificity and hypoxicinducibility of Epo gene expression; processes which are probably primarily controlled at the level of gene transcription.

Control of transcription is complex, and is not well understood. A comprehensive discussion is beyond the scope of this thesis. The reader is referred to excellent recent reviews (Mitchell and Tijan 1989; Tijan and Maniatis 1994; Buratowski 1994). The transcripton complex assembles in the promoter region of the gene, usually with a consensus TATA box, lying about 30 base pairs upstream of the transcription start site, which the highly conserved protein TFIID binds to. In genes such as Epo with no TATA box, TFIID is still involved in binding to DNA in the region of transcription initiation. The transcription complex consists of several proteins and RNA polymerase II which bind in a cooperative and sequential manner. Once the transcription complex has formed it is relatively stable. Promoter sequence in genes with a TATA box can be defined as sequence between the TATA box and the 
transcription start site. In genes such as the Epo gene which lack a consensus TATA box, it is functionally defined as sequence required to direct appropriately initiated transcription off a DNA template. Gene promoter regions may be tissue-specific in their action. Upstream of the promoter there are frequently elements found $5^{\prime}$ to a wide range of genes, CCAAT boxes (which can be bound by several different proteins) and Spl sites. These can be regarded as upstream promoter elements, and are important in increasing promoter activity over the level of the minimal promoter. In addition there may be sequences involved in regulated transcription; this can be demonstrated by transferring the sequence and hence the regulatory property to another gene or by inactivating the site and showing loss of regulation. Short elements upstream of the promoter can confer a high degree of tissue specificity. Frequently a degree of tissue-specific expression in transgenic animals can be achieved with a relatively short stretch of DNA from the 5 ' region, in contrast to the situation with Epo, where a distant 5' element is necessary for renal expression. However, there are very few examples where a combination of cis-acting sequences capable of producing complete fidelity of gene expression have been identified. This suggests that more distant elements, such as those identified for the $\beta$ globin locus are involved (Crossley and Orkin 1993).

Protein components of the transcription complex interact with other proteins, known as transcription factors (for review see Pabo and Sauer 1992). These factors usually interact with DNA, either in the promoter region or at a distance from it in enhancer regions. Alternatively, the interaction may be between the transcription factor and other proteins. Transcription factors appear to have a modular structure. A model transcription factor will consist of three components; a DNA binding domain, an activation domain and a protein binding domain. The DNA binding domain binds to a short sequence of DNA (typically 6 to 8 nucleotides). Although these binding sites fit a consensus sequence, there is often a degree of flexibility in the sequence requirement. Inherently this may seem curious; since a six nucleotide sequence (with no degeneracy) will occur every 2048 bp of random DNA. It is therefore not surprising that the binding of transcription factors to DNA is influenced by other factors; in particular chromatin structure and other proteins. An important corollary of this is that searching sequence for consensus binding sites will often produce a very large number of potential sites, and interpretation requires other data, such as functional studies or concerning chromatin structure. 
The protein-binding domains of transcription factors allow interaction with other transcription factors, some of which may not bind to DNA directly. In many cases the formation of heterodimers or homodimers is important. At least in some cases there is an ability to form a range of different dimeric structures with members of a family of transcription factors; clearly this can increase the response repertoire very considerably. The activation domain interacts with a component of the transcription complex, hence influencing transcription. In inducible systems, a gene may be regulated at the transcriptional level by a broad range of mechanisms involving transcription factors. Since the number of transcription factors must be limited, it is not surprising that the diversity of gene expression appears to be generated by a large number of potential interactions between a much smaller number of transcription factors. A transcription factor may itself be regulated at the transcriptional level, thus forming a transcriptional cascade. Alternatively a transcription factor which is constitutively present may be modified to an active form which can then bind to DNA or interact with other transcription factors and/or the transcription complex. In other cases the transcription factor is in a cytoplasmic location and is able to move to the nucleus when another protein is removed.

The transcriptional response to hypoxia resulting in increased expression of the Epo gene is not well understood. Two well-characterised examples of inducing gene expression via transcriptional responses are heat shock (Schlesinger 1990) and steroid hormones (Beato 1989). In the case of the heat shock response, a transcription factor which does not constitutively bind DNA is modified by the stimulus permitting it to bind to a site that is already exposed (ie hypersensitive) upstream of the promoter in a number of genes. The bound transcription factor also needs to be phosphorylated to reveal an activation domain. Heat shock activates a range of genes in this fashion via cis-acting elements, and the system is ubiquitous. In the case of glucocorticoids the receptor which binds the hormone is itself a transcription factor. It is usually bound to another protein (Hsp90) which is displaced when the hormone binds to its receptor. This displacement allows the receptor to bind DNA, and as it does so it forms a hypersensitive site, allowing the binding of other transcription factors. Again, a wide range of genes contain glucocorticoid response elements and are modulated by this transcription factor. Steroid receptors illustrate another important point; there is a whole family of steroid receptors with similar structures and different consensus sequences. Many other such families of transcription factors are known. 
Regulation of gene transcription involves not only proteins which interact directly or indirectly with DNA motifs, but also the structure of the DNA. Thus changes in chromatin structure (Felsenfeld 1992) and methylation (Tate and Bird 1993) are also important. Clearly in order to study such effects a cell population which is expressing the gene at a consistent level is necessary. Thus in the case of Epo this could be studied in hepatoma cells, but would be unlikely to be helpful in whole liver or kidney given the small proportion of cells expressing the gene.

Many experimental techniques have been used to investigate the regulation of gene expression. In this thesis transgenic mice and transient transfection of tissue culture cells have been used to study regulation of the Epo gene. In the transgenic mice, DNA from the Epo locus will have included multiple cis-acting elements (whose precise location is not known) and the DNA will have been subject to methylation and the effects of chromatin structure. By using extensive sequence, many aspects of Epo regulation were emulated in the transgenic mice. This approach has been used to investigate aspects of Epo gene expression which are likely to rely on interactions between multiple regulatory mechanisms. In particular it has been used to identify cells responsible for Epo gene expression in liver and kidney.

In terms of understanding regulation of the Epo gene one important aim is to define the mechanism of the transcriptional response to hypoxia. One possibility is that there is a molecular oxygen-sensor, which may be a haem protein. This might act via a transcriptional cascade, such as that found in nitrogen-fixing bacteria. Alternatively it is possible that the response is due to the interaction of several transcriptional control systems, rather than being governed by a single specific oxygen-regulated system. An interesting issue is whether the oxygen-sensing mechanism is tissue-restricted (to Epo producing cells), or is more widespread. In the latter event the tissue-specificity of Epo production would be superimposed on a general oxygen-sensing mechanism. Transient transfection of tissue culture cells allows the study of cis-acting elements without extensive sequence (i.e. in isolation from other elements) and without the effects of chromatin structure. Such experiments might allow the study of some aspects of Epo regulation in relative isolation. In this thesis it has been used to study gene regulation by hypoxia independently from other aspects of Epo gene regulation (such as tissue-specificity). Transient transfection of the Epo 3' enhancer followed by exposure to hypoxia (a reduction in $\mathrm{pO}_{2}$ in the incubator) was applied to a range of cell types. The hypoxic response has distinctive features which are of 
assistance in distinguishing this regulatory response from other systems, and support its use as a model for studying regulation of the Epo gene. In particular the response to hypoxia is also induced by cobalt, is not influenced or induced by cyanide and is abrogated by cycloheximide.

\subsection{PRINCIPAL ISSUES ADDRESSED IN THIS THESIS}

The demonstration of a transcriptional control system responding to hypoxia and regulating expression of the Epo gene raises several issues. In chapter 3 the possibility is tested that this system (or a very closely related one) is not restricted to controlling Epo gene expression but is operating more widely.

In chapters 4 to 6 the major experimental aim was to characterise the cells producing Epo in the kidney and liver. In order to do this, sequence from the Epo locus was used to direct expression of a marker gene, SV40 T antigen, to the Epo-producing cells.

Striking features of Epo gene expression in the kidney are the reduced expression in renal disease, and the failure of explanted cells to express the gene in tissue culture. This is the subject of Chapter 7.

In the course of the transgenic experiments, a targeted modification of the mouse Epo gene occurred. Homozygotes for the resultant allele had a greatly reduced capacity for Epo gene expression. Chapter 8 is involved with this recombinant allele and describes the effects of severe Epo deficiency. In addition, the possibility is investigated that further targetted modifications of the mouse genome in this region could be engineered using a similar experimental design. 


\section{Methods}

\subsection{RNA ANALYSIS BY RNase PROTECTION}

The majority of the experimental work described in this thesis is concerned with measuring gene expression. This has involved either detection of mRNA or, in the case of some transgenic experiments, the accumulation of a marker protein in cells. For mRNA analysis, total RNA was subjected to RNase protection with specific antisense probes. The principles of this technique are the same for assessment of transcripts from transiently transfected reporter genes, endogenous genes in tissue culture cells, endogenous genes in mice and mouse transgenes. The methods of RNA extraction, the amount of RNA assayed, the probes employed and the hybridisation conditions varied considerably.

Measuring mRNA has the advantage that the measured quantity depends only on gene transcription and stability. Changes will occur in advance of the accumulation or secretion of a gene product and will not be altered by variables such as translational efficiency. Several methods are available for mRNA detection; RNase protection has the advantage of high sensitivity (exceeded only by reverse-transcriptase PCR detection). Although this was not generally important when assessing transiently transfected reporter genes, it was vital for detection of Epo mRNA in the tissues of unstimulated mice. Furthermore, with appropriate probe design it is specific; cross-hybridisation to other species being negligible. The probes employed were designed such that the full-length probe included sequence which was either not complementary to the mRNA (vector sequence or non-transcribed sequence from genomic DNA) or crossed from one exon to another (if cloned from a cDNA). Detection of the predicted length fragment from the original probe thus provided a specific and robust assay system. The assays were highly reproducible, and when combined with liquid scintillation counting allowed quantitative measurements of mRNA species (Potter et al. 1991). An additional advantage of RNase protection analysis was that it was usually possible to design probes such that two different mRNA species were detected in the same assay, thus controlling for several factors (e.g. transfection efficiency and RNA recovery) throughout the experiment. Finally, by using probes which crossed the CAP site, it was possible to assess the site of initiation of the transcripts, which was important both in the transient expression experiments and in animal experiments. 
The RNase protection assay employed was a modification of that described by Melton et al (Melton et al.1984). In principle, the technique involved hybridising the RNA to be analysed with a ${ }^{32} \mathrm{P}$-labelled antisense riboprobe(s). RNase was then added; this digests the RNA except where an RNA-RNA hybrid has formed. Subsequently protected fragment(s) of the probe were analysed by denaturing polyacrylamide gel electrophoresis.

\subsubsection{Protocol for RNA extraction from tissue culture cells}

Total RNA was prepared from tissue culture cells using a commercially obtained guanidinium-phenol extraction method (RNAzolB, Biogenesis, Bournemouth). All cells and subsequent samples from an experiment were handled in parallel. Disposable plasticware and DEPC treated solutions were used to minimise the possibility of RNase contamination, and the steps were performed rapidly and where applicable on ice. Following the test period, adherent tissue culture cells were washed twice with ice-cold Hanks' balanced salt solution (HBSS) and then removed from the dish with trypsin/EDTA (Sigma, Poole) followed by ice-cold PBS. They were then centrifuged at 3,000 rpm in a Beckman GPR centifuge (Beckman Instruments Inc., Palo Alto, USA) for 5 minutes at $4^{\circ} \mathrm{C}$, and the cell pellet was resuspended in 500 or $750 \mu 1$ RNAzolB. Subsequent steps were performed according to the manufacturer's instructions. RNA was finally dissolved in 30 or $50 \mu \mathrm{l}$ RA hybridisation buffer (80\% formamide, $40 \mathrm{mM}$ piperazine-N,N'-bis(2ethanesulphonic acid) (PIPES), 400 $\mathrm{mM}$ sodium chloride, $1 \mathrm{mM}$ EDTA, $\mathrm{pH} 8$ ) by heating at $95^{\circ} \mathrm{C}$ for ten minutes, and stored in this form at $-20^{\circ} \mathrm{C}$.

\subsubsection{Protocol for RNA extraction fronı animal tissues}

Animals were killed by cervical dislocation. Organs were rapidly removed. Surgical instruments were cleaned between dissection of each organ to minimise RNA carryover. Disposable plasticware and DEPC treated solutions were used throughout. The tissue (either a portion of approximately $300 \mathrm{mg}$, or if less than this size the whole organ or pair of organs) was placed in $7.5 \mathrm{ml}$ of $4 \mathrm{M}$ guanidinium thiocyanate/10mM EDTA $/ 50 \mathrm{mM}$ Tris (pH7.5) with either $0.7 \mathrm{M} \beta$-mercaptoethanol or $1 \mathrm{mM}$ DTT as a reducing agent (a direct comparison showed no difference and DTT was preferred for handling). Homogenisation was performed for 30 to 60 seconds with an Ultra Turrax T25 homogeniser (Janke \& Kunkel IKA Labortechnik, Stanfeni, Germany) operated at full speed. Following homogenisation the probe was cleaned with $3 \%$ hydrogen peroxide, followed by ethanol, three washes in DEPC treated water and a final rinse in guanidinium thiocyanate. The homogenates were then centrifuged at 3,750 rpm in a Beckman GPR centrifuge to remove particulate material, 
and $6.5 \mathrm{ml}$ supernatant removed. These homogenates were stored at $-80^{\circ} \mathrm{C}$ prior to further analysis. For purification of RNA $0.5 \mathrm{~g} \mathrm{~N}$-lauroylsarcosine and $3 \mathrm{ml} 5.7 \mathrm{M}$ caesium chloride solution was added to the thawed homogenate. This was then centrifuged at $3,750 \mathrm{rpm}$ for 15 mins in a Beckman GPR centrifuge and the supernatant layered onto a $4 \mathrm{ml}$ caesium chloride cushion in a polyallomer ultracentrifuge tube. Samples were then ultrcentifuged at 32,000 rpm for 22 hours in a Beckman LM-8 ultracentrifuge fitted with an SW40Ti rotor.

The caesium chloride gradient and cushion were then removed from the tube, leaving the RNA pellet on the bottom. The bottom of the tube was then cut off and the RNA dissolved in $360 \mu 1$ of $10 \mathrm{mMTris} \mathrm{pH} 7.5 / 1 \mathrm{mM}$ EDTA/0.2\% SDS. This solution was heated at $55^{\circ} \mathrm{C}$ for 3 minutes, prior to precipitation by addition of 0.1 vol. $3 \mathrm{M}$ sodium acetate $(\mathrm{pH} 5.5)$ and $900 \mu \mathrm{l}$ ethanol. The RNA pellet was dissolved in 50 to $200 \mu \mathrm{l}$ in RNA hybridisation buffer by heating to $95^{\circ} \mathrm{C}$ for 10 to 20 minutes with repeated mixing. The RNA was then stored at $-20^{\circ} \mathrm{C}$.

\subsubsection{RNase protection assays}

RNA samples were quantitated by absorbance measurements at $260 \mathrm{~nm}$ using a Beckman DU-62 spectrophotometer. The quantity of total RNA to be assayed was then brought up to a standard volume for the assay ( 30 or $50 \mu \mathrm{l}$ ) with RNA hybridisation buffer. The antisense labelled riboprobe(s) $\left(2.5 \times 10^{5} \mathrm{cpm}\right.$ for endogenous genes, $5 \times 10^{5} \mathrm{cpm}$ for transiently transfected genes) were added. The samples were denatured at $95^{\circ} \mathrm{C}$ for 15 minutes prior to overnight hybridisation $\left(55^{\circ} \mathrm{C}\right.$ or $60^{\circ} \mathrm{C}$, see below). The following morning $360 \mu 1$ RNase $\mathrm{A}$ $(40 \mu \mathrm{g} / \mathrm{ml})+$ RNase T1 $(2 \mu \mathrm{g} / \mathrm{ml})$ in RNase buffer $(10 \mathrm{mM}$ Tris pH7.5, 5mM EDTA, 300 $\mathrm{mM}$ sodium chloride) was added to the samples and they were incubated at $25^{\circ} \mathrm{C}$ for thirty minutes. $50 \mu \mathrm{l}$ of Proteinase $\mathrm{K}$ solution ( $1 \mathrm{mg} / \mathrm{ml}$ in RNase buffer) and $10 \mu \mathrm{l}$ of $20 \%$ SDS $\left(\mathrm{w} / \mathrm{v}\right.$ in $\mathrm{dH}_{2} \mathrm{O}$ ) were added and samples incubated at $37^{\circ} \mathrm{C}$ for 30 minutes. Protein was then extracted by vortex mixing with an equal volume of phenol:chloroform for fifteen seconds, separating in a micro-centrifuge (five minutes) and removing the aqueous phase to a fresh tube. Residual phenol was extracted by a similar extraction using chloroform. The aqueous phase $(360 \mu \mathrm{l})$ was precipitated by the addition of $2.5 \mathrm{vol}$. absolute ethanol and freezing in solid $\mathrm{CO}_{2}$. If less than $50 \mu \mathrm{g}$ total RNA was being analysed, $20 \mu \mathrm{g}$ of tRNA in DEPC treated water was added prior to this to ensure complete precipitation. Samples were then allowed to thaw slowly at $-20^{\circ} \mathrm{C}$ for 30 minutes followed by centrifugation in a microcentrifuge at $4^{\circ} \mathrm{C}$. The resultant pellet was dissolved in $5 \mu 1$ of electrophoresis buffer ( $1 \mathrm{x}$ TrisBorate-EDTA buffer, $80 \%$ formamide with bromophenol blue and xylene cyanol) by heating 
to $95^{\circ} \mathrm{C}$ for 15 minutes, and the denatured samples were then loaded onto a $0.5 \mathrm{~mm} 8 \%$ or $10 \%$ denaturing polyacrylamide gel (Sequagel, National Diagnostics, Hull) which had been pre-warmed for 45 minutes. PBR322 DNA digested with HpaII and labelled by Klenow infill with ${ }^{32} \mathrm{P}$ dCTP was loaded as a size marker. Electrophoresis in TBE was carried out under power-limited conditions. The gel was then fixed in a solution containing $10 \%$ acetic acid and $10 \%$ methylated spirit for 30 minutes, dried on a gel dryer (BioRad Laboratories, Richmond, U.S.A.) and placed in an X-ray cassestte with intensifying screens against radiographic film for 1 hour to 10 days, depending on the strength of signal.

\subsubsection{Quantitation of specific mRNA species}

In order to quantitate specific mRNA species, bands were excised from the polyacrylamide gel and the level of radioactivity determined using a liquid scintillation counter. This method was developed for quantification of Epo mRNA from rat tissues and has been validated previously (Potter et al. 1991). It is sensitive, with low background, and allows quantification of a large number of samples. To identify bands for excision, the gel was aligned with an autoradiograph using markers. At the level of the band to be analysed an equal size area was marked in each lane using a template. These areas were then excised from the gel and placed in alternating positions between two mats impregnated with scintillation fluid. They were then counted in a multichannel flatbed scintillation counter (model 1205 Betaplate; Pharmacia-Wallac OY, Turku, Finland). The counting parameters were set so that each sample position was counted for 60 seconds, or until the variance was less than $1 \%$. The counts detected from the lane in which a negative control (no RNA) sample was loaded were subtracted from the resultant counts, to correct for background.

\subsubsection{Riboprobes}

The specific riboprobes employed are summarised in Table 2.1. In each case, the DNA fragment was cloned into a suitable plasmid vector in the correct orientation to produce antisense transcripts from the plasmid SP6 promoter. Not included in the table are the two $\alpha_{1}$-globin riboprobes, $\alpha 98$ and $\alpha 132$ which crossed the cap site of the human $\alpha_{1}$-globin gene and protected 98 and 132 bp respectively (these probes were a gift from Dr R. W. Jones). 


\begin{tabular}{|l|l|l|l|l|}
\hline \multicolumn{1}{|c|}{ Probe Name } & Vector & \multicolumn{1}{c|}{ Insert } & $\begin{array}{l}\text { Linearisation } \\
\text { Enzyme }\end{array}$ & \multicolumn{1}{|c|}{ Protected lengths } \\
\hline $\begin{array}{l}\text { Human growth } \\
\text { hormone }\end{array}$ & pSP64 & Sac1-Pst1(includes exon 3) & EcoR1 & 117 \\
\hline Mouse Epo exon 1 & pGem7 & Xbal-Sac1(1-622) & Xbal & $\begin{array}{l}213(\text { Epo), 65 (Epo-TAg) } \\
148+65(\text { Epo-M) }\end{array}$ \\
\hline Epo-M & pGem7 & $\begin{array}{l}\text { Xbal-Sac1(1-622)+8bp at } \\
474\end{array}$ & Xba1 & $\begin{array}{l}221(\text { Epo-M) } \\
148+65(E p o)\end{array}$ \\
\hline Epo-TAg & pAM18 & $\begin{array}{l}\text { Xbal-Stu1(Epo1- } \\
476)+S V 40(5243-5194)\end{array}$ & Xbal & $\begin{array}{l}120(\text { Epo-TAg) } \\
65(\text { Epo) }\end{array}$ \\
\hline Human Epo cDNA & pAM18 & $\begin{array}{l}\text { Hind2-Pst1(exon3/1626- } \\
\text { exon4/2383) }\end{array}$ & Hind3 & 147 \\
\hline C Hamster Epo & pSP72 & PCR frag in at EcoRV, Pst1 & $\begin{array}{l}\text { Bgl2 } \\
\text { Dde1 }\end{array}$ & $\begin{array}{l}140+60 \\
140\end{array}$ \\
\hline Rat Epo exon 5 & pSP64 & Sac1-Pst1(450bp) & EcoR1 & 132 \\
\hline
\end{tabular}

Table 2.1 - Riboprobes employed for RNA analysis.

Plasmid DNA was prepared by alkaline lysis and caesium chloride ultracentrifugation. 50 $\mu \mathrm{g}$ of plasmid DNA was then linearised with the appropriate restriction enzyme, resulting in a 5 ' overhang. The DNA was then subjected to phenol:chloroform and chloroform extraction, followed by ethanol precipitation. After washing with $70 \%$ ethanol and drying, the pellet was dissolved in $35 \mu 1 \mathrm{RNase}$ free Tris EDTA, and stored at $-20^{\circ} \mathrm{C}$. An aliquot was subjected to electrophoresis to check that it was completely linearised and that the concentration was approximately $1 \mu \mathrm{g} / \mu \mathrm{l}$.

In order to generate continuously labelled antisense riboprobes, $1 \mu$ template was mixed with $2 \mu 15 \times$ SP6 reaction buffer, $0.25 \mu l$ of each of UTP, ATP, CTP (each at $20 \mathrm{mmol} / \mathrm{l}$ ), 5.0 $\mu \mathrm{l} 32 \mathrm{P} \alpha$-GTP (specific activity $400 \mathrm{Ci} / \mathrm{mmol}$; Amersham International, Amersham); then $0.8 \mu 1$ human placental ribonuclease inhibitor (Boeringher Mannheim UK, Lewes) and $1 \mu 1$ SP6 RNA polymerase (International Biotechnologies Inc, New Haven, USA) were added. The reaction was incubated for 60 to 90 minutes at $37^{\circ} \mathrm{C}$. $2 \mu \mathrm{l}(10 \mathrm{U}) \mathrm{RNase}$-free DNase 1 (Pharmacia LKB Biotechnology, Milwaukee, Wisconsin) was then added and the mix was incubated for a further 20 minutes at $37^{\circ} \mathrm{C}$ in order to digest the DNA template. $87 \mu 1$ of 10 $\mathrm{mM}$ Tris $\mathrm{HCl} / 5 \mathrm{mM}$ EDTA/0.1\% SDS was then added and the sample spun through a preequilibriated G50 sephadex column, to remove unincorporated nucleotides. RNA was precipitated by the addition of 0.1 vol $3 \mathrm{M}$ sodium acetate ( $\mathrm{pH} 5.2$ ), $30 \mu \mathrm{g}$ tRNA and 2.2 vol. absolute ethanol, freezing in solid $\mathrm{CO}_{2}$, allowing to thaw at $-20^{\circ} \mathrm{C}$ and then centrifuging in a microcentrifuge for 15 minutes at $4^{\circ} \mathrm{C}$. The pellet was then resuspended in $100 \mu \mathrm{l}$ RNA hybridisation buffer by heating at $95^{\circ} \mathrm{C}$ for 10 minutes and an aliquot was counted using a liquid scintillation counter (Packard Instruments BV, Gronigen, Holland). $50 \times 10^{6}$ to 120 $x 10^{6} \mathrm{cpm}$ total incorporation was reliably obtained by this method. The probe was then stored at $-20^{\circ} \mathrm{C}$. Probes were not used for more than one week after synthesis, to avoid loss of sensitivity and fragmentation by radiolysis. 


\subsection{MAINTENANCE OF ESTABLISHED CELL LINES}

A wide range of tissue culture cell lines were used in this experimental work. The cell lines, the source from which they were obtained and the specific culture conditions employed are shown in Table 2.2. Culture medium was supplemented with penicillin, streptomycin and glutamine. Foetal calf serum was not heat-inactivated unless specifically indicated. All cells were grown in bicarbonate buffered media in a $5 \% \mathrm{CO}_{2}$ incubator, in disposable Falcon flasks (Becton Dickinson UK, Cowley) with loosened tops. Culture media were obtained from Hyclone, Cramlington.

\begin{tabular}{|c|c|c|c|c|c|}
\hline Cell line & Species & Source & Medium & FCS & Adherent \\
\hline U937 & Human & ECACC & RPMI & $10 \% *$ & - \\
\hline MRC5 & Human & ECACC & EMEM $^{1}$ & $10 \%$ & + \\
\hline IBR3 & Human & ECACC & EMEM $^{1}$ & $15 \%$ & + \\
\hline GN4 & Human & S Povey & EMEM $^{2}$ & $10 \%$ & + \\
\hline HepG2 & Human & ECACC & EMEM & $10 \%$ & + \\
\hline Hep3B & Human & ECACC & EMEM & $10 \%$ & + \\
\hline $\cos 7$ & Simian & ECACC & DMEM & $10 \%$ & + \\
\hline BACE & Cow & R Bicknell & DMEM $^{3}$ & $10 \%$ & +4 \\
\hline LLCPK1 & Pig & ECACC & 199 & $10 \%$ & + \\
\hline FAZA & Rat & S Povey & EMEM $^{2}$ & $10 \%$ & + \\
\hline NRK49F & Rat & ECACC & DMEM $^{1}$ & $5 \%$ & + \\
\hline NRK52E & Rat & ECACC & $\mathrm{DMEM}^{1}$ & $10 \%$ & + \\
\hline$\overline{\mathrm{RAG}}$ & Rat & S Povey & EMEM $^{2}$ & $10 \%$ & + \\
\hline RAEC & Rat & A Ager & RPMI & $10 \%$ & + \\
\hline $\mathrm{a} 23$ & C Hamster & S Povey & EMEM $^{2}$ & $10 \%$ & + \\
\hline $\mathrm{K} 1$ & C Hamster & S Povey & EMEM $^{2}$ & $10 \%$ & + \\
\hline V79 & C Hamster & S Povey & EMEM $^{2}$ & $10 \%$ & + \\
\hline MEL (585) & Mouse & W Wood & RPMI & $15 \%$ & - \\
\hline MEL (707) & Mouse & R Jones & RPMI & $15 \%$ & - \\
\hline $\mathrm{J} 774.2$ & Mouse & ECACC & DMEM & $10 \% *$ & - \\
\hline
\end{tabular}

Table 2.2. - Tissue culture cells, sources and culture conditions. ${ }^{1}$ supplemeted with $1 \%$ nonessential amino acids. ${ }^{2}$ supplemented with ImM sodium pyruvate. ${ }^{3}$ supplemented with heparin, bovine brain extract, HEPES. ${ }^{4}$ plates pretreated with $1 \%$ gelatin overnight. * Heatinactivated at $56^{\circ} \mathrm{C}$ for 30 minutes.

Regardless of rate of growth, adherent cells were passaged when they reached confluence, by rinsing with HBSS and digesting with trypsin/EDTA. Fresh culture medium was added and the cells were then subjected to vortex mixing to separate multicellular aggregates. The cells were then divided into new flasks. In the case of HepG2 cells, which tend to form clumps, care was taken to passage the cells frequently, to mix the cells very vigorously follwing trypsinisation, and to seed cultures at rather low density. Medium was changed over growing cultures at approximately weekly intervals. Cells grown in suspension were passaged when the indicator changed colour indicating that the culture was becoming acidotic. An aliqot of the culture was then added to fresh medium. 
Precautions were taken to avoid contamination of cell cultures either by micro-organisms or by other cell lines. Separate medium was kept for each cell line, and different cell lines were not handled in the cabinet simultaneously. Cultures were examined regularly to check the morphology of the cells. The cells were not routinely monitored for mycoplasma infection.

\subsection{TRANSIENT TRANSFECTION OF DNA}

DNA used for transient transfection studies was in the form of supercoiled plasmid DNA prepared by standard techniques on caesium chloride gradients; in general two plasmids were transfected, one acting as a control for transfection efficiency and RNA recovery. Cells were approaching confluence prior to transfection, unless otherwise stated. In general, one $35 \mathrm{~cm}^{2}$ tissue culture flask with a confluent monolayer of cells was used for each individual electroporation. Cells were harvested for transfection as follows; the flasks were washed twice with HBSS, and then the cells released with trypsin/EDTA. Cells were pelleted and then resuspended by vortex-mixing in sufficient RPMI 1640 medium (with no additives) for $0.8 \mathrm{ml}$ per transfection. This suspension was then divided into $0.8 \mathrm{ml}$ aliquots in plastic cuvettes, and the DNA (dissolved in $200 \mu$ l of PBS) was added. The cells were then kept on ice, and resuspended again gently immediately prior to transfection.

Transfection was performed by electroporation using a purpose-built $4 \mu \mathrm{F}$ capacitor array charged with a high voltage power supply. The charging voltage was optimised for each cell line in preliminary experiments. The electric current was delivered through platinum electrodes, which fitted inside the cuvette. After electroporation the cells were placed on ice for ten minutes and then diluted into an appropriate volume $\left(8 \mathrm{ml}\right.$ per $\left.75 \mathrm{~cm}^{2} \mathrm{dish}\right)$ of the standard culture medium for that cell line. This cell suspension was then divided into $8 \mathrm{ml}$ aliquots, any test chemicals were added to the appropriate aliquots and the $8 \mathrm{ml}$ aliquots of cell suspension were placed in a $75 \mathrm{~cm}^{2}$ culture dish. To obtain sufficient transfected cells on occasions where more than three parallel conditions were assessed, two electroporations were pooled, mixed and then divided. The dishes were then incubated for sixteen hours either under normoxic conditions $\left(5 \% \mathrm{CO}_{2}\right.$, balance air, humidity $\left.85 \%\right)$ or hypoxic conditions (see below). After the test period the cells were harvested for preparation of RNA. The number of cells transfected was quantitated by counting the number of cells in an aliquot of the pool used for the transfections, using a haemacytometer (Weber Scientific, Teddington). 


\subsection{HYPOXIC INCUBATION}

Cells were exposed to hypoxia in a Napco 7100 incubator (Precision Scientific, Chicago, USA). The incubator was set to deliver $5 \% \mathrm{CO}_{2}$, a controlled level of oxygen $(0.1 \%, 0.5 \%$, $1.0 \%$ or $3.0 \%$ ) with the balance consisting of nitrogen. Humidity was maintained at $85 \%$, and temperature at $37^{\circ} \mathrm{C}$. Transfected cells were handled as described above. Non-transfected cells were placed in the incubator in flasks with the lid removed or on $175 \mathrm{~cm}^{2}$ dishes. The cells had usually been divided from a single pool 24 to 72 hours previously, for parallel normoxic and hypoxic exposure. Immediately prior to exposure the medium was replaced with $15 \mathrm{ml}$ fresh medium per flask or dish.

\subsection{GENERATION OF TRANSGENIC MICE}

The transgenic experiments described in this thesis were performed in collaboration with Professor Martin Johnson's group in the Department of Human Anatomy at Cambridge University. The Epo-M and Epo-TAg constructs were made by Dr Peter Ratcliffe. All zygote injections, reimplantation and initial breeding from transgenic founders was performed in the Cambridge laboratory.

\subsubsection{DNA constructs}

DNA was prepared from a bacteriophage clone isolated from a BALB/c mouse genomic library in $\lambda E M B L 3$ (Clontech, Palo Alto, USA). The $16.5 \mathrm{~kb}$ insert ( $\lambda 3 \mathrm{a}$ ) contained the mouse Epo gene together with $9 \mathrm{~kb}$ of $5^{\prime}$ flanking sequence and approximately $3.5 \mathrm{~kb}$ of $3^{\prime}$ flanking sequence (Figure 2.1). This DNA was released by digestion with Sall (and was therefore flanked on each side by a short stretch of sequence from the $\lambda$ vector) and cloned into pBluescriptSKII (Stratagene, La Jolla, USA) following which nested pairs of unique restriction enzyme sites were used in successive subcloning procedures to generate a 456 bp EagI-BamHI fragment in which the BalI site lying in the Epo 5' untranslated region 65 bp from the cap site was unique. This site was used for insertion of (a) BglII linkers CAGATCTG (Epo-M) or (b) a linkered fragment consisting of nucleotides 5235 to 2533 from the SV40 viral genome and containing the entire T Ag coding sequence and poly $\mathrm{A}$ addition sites (Epo-TAg). For Epo-D the Eagl-BamHI fragment containing Exon 1 of the Epo gene was replaced by a linker which contained $E c o R V$ and $X h o l$ restriction sites. The $16.5 \mathrm{~kb}$ Epo sequence was then reconstructed by successive religations into the recombinant plasmids used in the original subcloning procedures. 


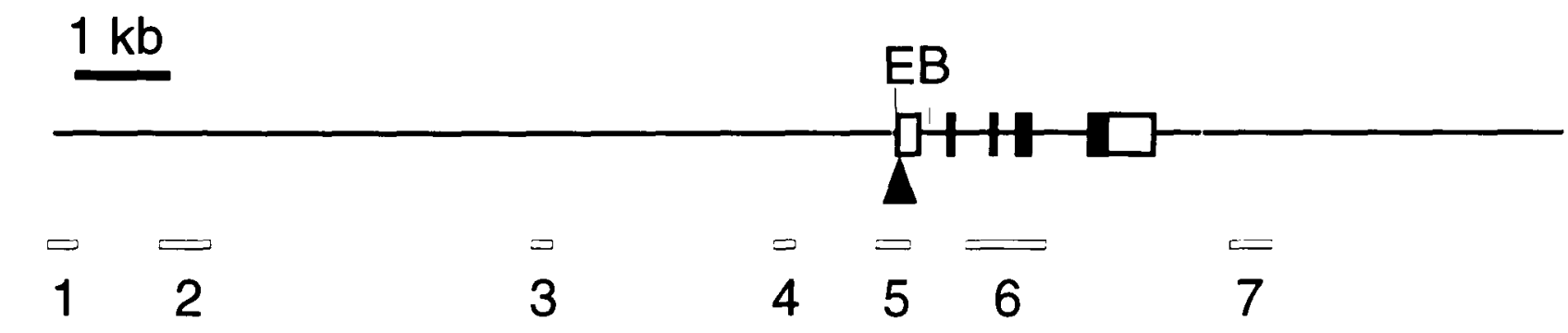

\begin{tabular}{|c|c|c|c|c|}
\hline Probe & $5^{\prime}$ & $3^{\prime}$ & Length(bp) & Compete \\
\hline 1 & Ava1 & Ava1 & 500 & Yes \\
\hline 2 & Kpn1 & Ava1 & 700 & Yes \\
\hline 3 & Pst 1 & Sst 1 & 300 & No \\
\hline 4 & Bgl2 & Pst 1 & 300 & No \\
\hline 5 & Xba1 & Bgl2 & 500 & No \\
\hline 6 & Pst 1 & Pst 1 & 1100 & No \\
\hline 7 & Apa1 & Pst 1 & 600 & No \\
\hline
\end{tabular}

Figure 2.1 - The $\lambda 3$ a insert (length $16.5 \mathrm{~kb}$ ) is shown schematically. Exons of the Epo gene are solid and the 5' and 3'UTR is open. The EagI (E) and BamHI (B) sites which were made unique by subcloning manoeuvres are indicated. In Epo-D, the Eagl and BamH1 sites were joined by a linker sequence. The Ball site used to insert BgllI linkers for Epo-M and $2.7 \mathrm{~kb}$ of SV40 sequence for Epo-TAg is indicated by an arrowhead. The locations of DNA probes (17) and the restriction enyme sites defining them are indicated. For probe 5 the BgllI site at the 3' end is the introduced BglII site of Epo-M. Where indicated sonicated mouse DNA (final concentration $200 \mu \mathrm{g} / \mathrm{ml}$ ) was added to the labelled probe prior to denaturation in order to reduce background hybridisation.

The sequence of the promoter and transcription start site for Epo-M, Epo-TAg and Epo-D is given in Appendix 2.

\subsubsection{Preparation of DNA for injection}

Plasmid DNA was prepared in DH5 $\alpha$. Plasmid DNA was recovered from a $250 \mathrm{ml}$ culture using a standard alkaline lysis protocol and was then purified by ultracentrifugation on a caesium chloride gradient with ethidium bromide. Following extraction of the ethidium bromide and precipitation, the insert was released by digestion with SalI and separated from the vector by agarose electrophoresis in TAE buffer without ethidium bromide. An aliquot was run in an adjacent lane, and this portion of the gel was then cut off and stained with ethidium bromide to allow the position of the DNA in the gel to be assessed. The corresponding area of the unstained gel was excised and the DNA electroeluted. The DNA was then precipitated with ethanol, washed with $70 \%$ ethanol and dialysed against $10 \mathrm{mM}$ Tris pH7.5, 0.1 mM EDTA.

The transgenic rate using the Epo-M and Epo-TAg constructs was low. Possible factors were considered to be the exposure to ethidium on the caesium chloride gradient; freezing and thawing the DNA during preparation or storage and the presence of impurities in the final solution. In view of this, the Epo-D construct was prepared on a caesium chloride gradient without ethidium, and was not frozen at any stage. In addition, prior to injection 
the DNA was passed over a G50 sephadex column. The final DNA solution was ultracentrifuged at $45,000 \mathrm{rpm}$ for ten minutes to remove any particulate matter and the supernatant was then decanted to a fresh tube and stored at $4^{\circ} \mathrm{C}$, in order to avoid repeated freezing and thawing.

\subsubsection{Recovery and handling of oocytes}

Female C57BL/10 x CBA mice were super-ovulated and mated with CFLP males (Interfauna, Huntingdon, $\mathrm{UK}$ ) to provide zygotes for injection. Male pronuclei were pressure-injected with DNA as described (Nasr-Esfahani et al. 1990). Injected zygotes were cultured overnight and two-cell embryos were transferred to oviducts of recipient females that had mated with vasectomised males the previous night.

\subsection{BREEDING AND MAINTENANCE OF MICE}

MF1 mice (a non-inbred albino strain obtained from Harlan Olac, Bicester) were used in the experiments to determine the distribution of Epo expression in normal mice. In addition, these mice or CFLP mice (Interfauna, Huntingdon) were used to maintain transgenic lines. Some transgenic mice were crossed with the mutant strain Nan, which has a haemolytic anaemia. This introduced genes from the inbred strains $\mathrm{C} 3 \mathrm{H} / \mathrm{HeH}$ and $101 / \mathrm{J}$ into the colony. Transgenic lines were managed in the same way as normal mice. Mice were reared in standard cages and fed on a standard rodent diet (Rodent Toxicology diet, Bantin and Kingman) with free access to tapwater. Diagnosis of transgenesis was by tail biopsy under general anaesthetic at 3 to 4 weeks of age; each mouse was marked at this point with earclipping and toe-clipping so that it was subsequently identifiable. As a general rule, PCR was used to diagnose the presence of a transgene in the mice. However, mice selected for subsequent breeding were always diagnosed by restriction digestion and Southern analysis to ensure that they were transgenic and that they were from the correct transgenic line.

The zygote injections were performed at Cambridge, and at a subsequent point each line was transferred to a "clean" colony in Oxford by embryo transfer into pseudopregnant females. In addition, some animals were also transferred to a "dirty" colony in Oxford. No distinction is made between these groups of animals in the work described, nor was any difference in the animals observed. It is important to note that it became clear during the period of these experiments that other mice kept in the same facility in Cambridge were found to have mouse hepatitis virus and Sendai virus infection. Furthermore, a proportion 
of albino mice in all three colonies had a tendency to develop marked excoriation, and were found to be carrying mites (myobia musculi). In general the breeding efficiency of the colonies was acceptable, with an average litter size of eight offspring.

\subsubsection{Extraction of DNA}

Approximately one $\mathrm{cm}$ of tail was used. The tail samples were extracted within one week of biopsy, and in the interim period were stored at room temperature or $4^{\circ} \mathrm{C}$ in $700 \mu \mathrm{l}$ tail buffer (50mM Tris ( $\mathrm{pH} 8) / 100 \mathrm{mM}$ EDTA/100mM NaCl/1\% SDS). For digestion, the tails were finely minced with scissors, $35 \mu l$ Proteinase $\mathrm{K}$ solution $(10 \mathrm{mg} / \mathrm{ml}$ in water) was added and the samples incubated overnight in a shaking waterbath at $55^{\circ} \mathrm{C}$. The following morning a further $10 \mu \mathrm{l}$ Proteinase $\mathrm{K}$ solution was added and the samples incubated for a further 1 hour. The samples were then extracted twice with phenol:chloroform and once with chloroform. The DNA was then precipitated by adding an equal volume of propan-2ol. The resultant precipitate was washed with $70 \%$ ethanol and then air-dried on the bench for 30 minutes, before adding $50 \mu \mathrm{l} 10 \mathrm{mM}$ Tris ( $\mathrm{pH} 8$ )/1mM EDTA. Genomic DNA samples were stored at $4^{\circ} \mathrm{C}$.

\subsubsection{Transgenic diagnosis}

Epo-TAg, Epo-M and Epo-D transgenes could be diagnosed by a BglII digest (10 to $15 \mu \mathrm{g}$ DNA in $50 \mu 1$ of the manufacturer's buffer with $1.25 \mathrm{mM}$ spermidine), agarose electrophoresis on a $0.7 \%$ gel and Southern transfer to a positively charged membrane (Hybond N; Amersham International). DNA was cross-linked to the membrane with uv light (Stratalinker, Stratagene) followed by baking for 2 hours at $80^{\circ} \mathrm{C}$. Radiolabelled probe was generated from the $1.1 \mathrm{~kb}$ Pst 1 fragment (Probe 6) shown in Figure 2.1. Random nonamer primed probes were generated using a Megaprime kit (Amersham International). Membranes were pre-hybridised in DNA hybridisation buffer (6 x SSC / 5 x Denhardt's solution / 50\% formamide / $5 \%$ dextran sulphate / $1 \%$ SDS with $20 \mathrm{mg} / \mathrm{l}$ denatured ultrasonicated salmon sperm DNA) for six hours at $42^{\circ} \mathrm{C}$ in a hybridisation oven (Hybaid, Teddington) prior to addition of probe and overnight hybridisation. Membranes were washed at $55^{\circ} \mathrm{C}$ in $0.1 \mathrm{x}$ SSC/0.2\% SDS twice for 15 minutes and exposed to X-ray film.

Probe 6 hybridised to a $4.5 \mathrm{~kb}$ BgllI fragment in genomic DNA from MF1, CFLP, C57BL10 and CBA mice. Epo-M gave a fragment of $3.6 \mathrm{~kb}$, Epo-TAg a fragment of $6.3 \mathrm{~kb}$ and Epo$\mathrm{D}$ a fragment of $4.1 \mathrm{~kb}$. A naturally occurring polymorphism was found in Nan mice; loss of a $B g l I I$ site 3 ' to the gene giving a $250 \mathrm{bp}$ longer fragment hybridising to probe 6 (the $\mathrm{B}$ allele) when compared to the normal A allele. Further analysis showed that this allele 
originated from the inbred strain $101 / \mathrm{J}$, and was also present in the closely related strains $129 / \mathrm{SV}$ and 129/Ola. This B allele was used in breeding experiments to demonstrate that in the Epo-TAg ${ }^{\mathrm{H}}$ line a homologous recombination had occurred at the Epo locus. The positions of the BglII sites in each case are shown in Figure 2.2.

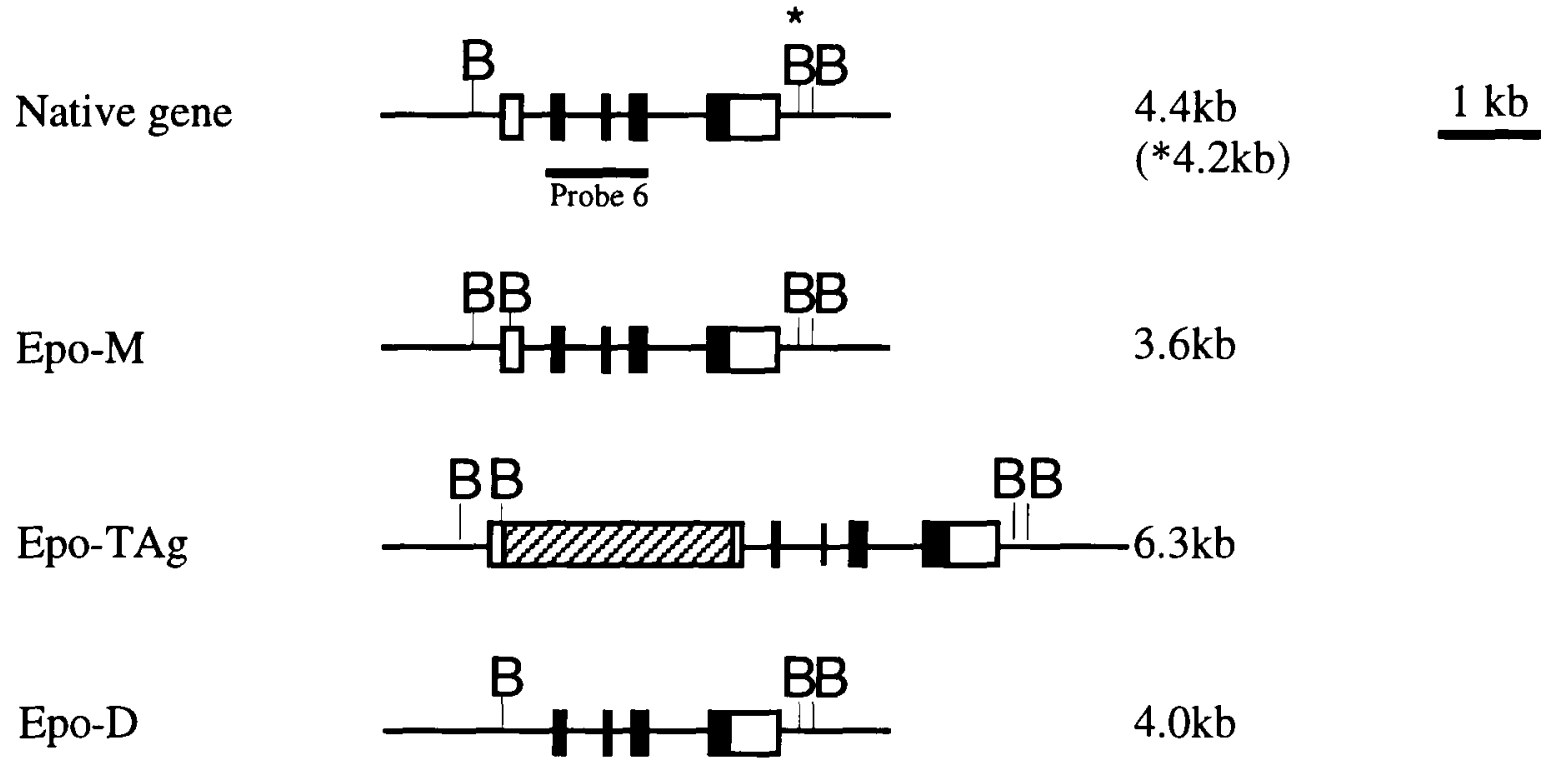

Figure 2.2 - The position of BglII restriction sites in the native Epo gene, the Epo-M, Epo-TAg and Epo-D genes is indicated. * indicates the polymorphic BglII site in the native Epo gene, which is not present in the 129/SV or 101/J strains of mice (the B allele). In each case the length of the fragment hybridising to Probe 6 is indicated.

Various other probes were used to define the precise arrangment and extent of integrated transgenic products, to examine for polymorphisms between different mouse strains and to screen a genomic cosmid library. The position of these probes is shown in Figure 2.1. With some probes, high background hybridisation (presumably due to the presence of repeat sequences) was observed. This was minimised by denaturing and hybridising the labelled probe in the presence of sonicated mouse genomic DNA (final concentration $200 \mu \mathrm{g} / \mathrm{ml}$ ).

\subsubsection{PCR diagnosis of transgenes}

Potential transgenic animals were screened for the presence of a transgene using PCR. A common 5' primer was used with the relevant transgene-specific 3' primer (Epo-M, EpoTAg and Epo-D). The positions of the primers are given in Appendix 2. In each case the reactions were performed in $12.5 \mu \mathrm{l}$, with $0.5 \mu \mathrm{g}$ tail DNA, $0.75 \mu \mathrm{M}$ each primer, $200 \mu \mathrm{M}$ each dNTP, $0.4 \mathrm{U}$ Taq polymerase (Promega) in the manufacturer's buffer. 33 cycles of amplification were performed, with an annealing temperature of $57^{\circ} \mathrm{C}$ for Epo-M and Epo$\mathrm{D}$ and $54^{\circ} \mathrm{C}$ for Epo-TAg. Samples were electrophoresed on 3\% agarose gels; Epo-M gave a band of $162 \mathrm{bp}$ and Epo-TAg a band of $120 \mathrm{bp}$. Epo-D gave a band of $121 \mathrm{bp}$, with a further band of $570 \mathrm{bp}$ from the endogenous Epo gene. 


\subsubsection{Acute haemolytic anaemia}

Mice were given intraperitoneal injections of $60 \mathrm{mg} / \mathrm{kg}$ phenylhydrazine freshly dissolved in HBSS, neutralised and passed through a $0.2 \mu \mathrm{m}$ filter. In general, three injections were administered at 36, 24 and 12 hours prior to sacrifice. This was found to result in a fall in the haematocrit to about half the normal value; the response was somewhat variable. For mice which were already anaemic (Epo-TAg ${ }^{\mathrm{H}}$ homozygotes) a single dose of $60 \mathrm{mg} / \mathrm{kg}$ was used. For polycythaemic mice up to four doses of $80 \mathrm{mg} / \mathrm{kg}$ were administered.

\subsubsection{Normobaric hypoxia}

Animals were placed in a lacquered gas-tight wooden box sufficiently large to contain two cages, constantly supplied with a mixture of nitrogen and oxygen. The flow-rate of each gas was determined by a rotameter (Meterate, Glass Precision Engineering Ltd, Herts., UK). The oxygen concentration in the box was monitored using an Oxychek oxygen meter (Critikon Inc., Florida, USA) and the gas flow adjusted so that the desired oxygen level was attained.

\subsubsection{Hypobaric hypoxia}

Animals were placed in a specially constructed air-tight metal chamber with a controlled leak mechanism (a gift from Dr P. M. Cotes). The lid of the chamber was perspex, allowing light to reach the mice. The chamber was connected to an electric pump. The pressure in the chamber was measured with an altimeter (Thommen, Switzerland) and recorded in terms of apparent altitude. The altitude achieved was altered by varying the air leak. The apparent altitude was increased in stages of no more than $2,000 \mathrm{~m}$ at intervals of 15 minutes; more rapid ascent was found to result in death.

\subsubsection{Measurement of haematocrit and venesection}

Blood samples were taken from a tail vein; or, if the animal was sacrificed, by cardiac puncture. Samples were analysed in heparinised glass capillary tubes, sealed with plasticine and spun in a micro-haematocrit centrifuge for 5 minutes (Hawksley, Lancing). When venesection was performed for serum Epo measurement or to exacerbate iron deficiency (see Chapter 7), the mice were warmed in a large beaker placed in a waterbath so that they were peripherally vasodilated. An incision was then made over a tail vein; between 200 and $500 \mu \mathrm{l}$ could be reliably removed by this method. 


\subsubsection{Measurement of serum Epo levels}

Serum samples were assayed for Epo by a double antibody RIA with delayed addition of trace (Cotes et al. 1989b). The first antibody was rabbit anti-human urinary Epo antiserum no. 7:139-18 (a gift from Dr G. K. Clemons). Tracer was ${ }^{125}$ I labelled recombinant human Epo (IM 178; Amersham International). The second antibody was a solid phase anti-rabbit Ig (Pharmacia decanting suspension no. 3). The standard curve for the assays was generated using the mouse serum standard, 83/589 (a gift from Dr P. M. Cotes). Samples and standard were diluted with BSA $50 \mathrm{mg} / \mathrm{ml}$ (Sigma, RIA grade) in PBS. The samples were assayed in duplicate, over a range of dilutions.

\subsection{IMMUNOLABELLING}

The principal antibodies used in immunolabelling studies are given in Table 2.3. A variety of different fixation methods were used to obtain the most suitable compromise between preserved antigenicity and structural integrity in these studies.

\begin{tabular}{|c|c|c|c|c|}
\hline Antigen & Clone & Description & Dilution & Reference \\
\hline CD45 & M1/9.3.4.HL2 & Rat mAb & neat $s / n$ & Springer et al. 1978 \\
\hline $\mathrm{F} 4 / 80 \mathrm{Ag}$ & $\mathrm{F} 4 / 80$ & Rat mAb & neat $s / n$ & Austyn and Gordon 1981 \\
\hline $\mathrm{F} 4 / 80 \mathrm{Ag}$ & - & Rabbit $\mathrm{a} / \mathrm{s}$ & $1: 1000$ & - \\
\hline Class II MHC (Ia) & B21-2 & Rat mAb & neat $s / n$ & Steinman et al. 1980 \\
\hline Thy 1 & YBM 29.2.1TG6 & Rat $\mathrm{mAb}$ & neat $s / n$ & Cobbold et al. 1983 \\
\hline fibroblast & ER-TR7 & Rat $\mathrm{mAb}$ & $1: 25$ & Van Vliet et al. 1984 \\
\hline PECAM/CD31 & MEC13.3 & Rat $\mathrm{mAb}$ & $1: 25$ & Vecchi et al. 1994 \\
\hline Fc receptor & $2.4 \mathrm{G} 2$ & Rat $\mathrm{mAb}$ & neat $s / n$ & Unkeless 1979 \\
\hline Cytokeratin & MNF116 & EPOS-mAb & neat & - \\
\hline Sm muscle actin & $1 \mathrm{~A} 4$ & EPOS-mAb & neat & - \\
\hline Desmin & D33 & EPOS-mAb & neat & - \\
\hline $\begin{array}{l}\text { Ecto-5'-nucleotidase } \\
\text { (5'NT) }\end{array}$ & - & Rabbit a/s & $1: 1000$ & Dawson et al. 1989 \\
\hline Factor VIII rAg & - & Rabbit a/s & $1: 200$ & - \\
\hline SV40 T Ag & - & Rabbit a/s & $1: 1000$ & - \\
\hline SV40 T Ag & PAb 101 (412) & Mouse $\mathrm{mAb}$ & neat $\mathrm{s} / \mathrm{n}$ & Gurney et al. 1980 \\
\hline SV40 T Ag & PAb 416 (L16) & Biotin mouse $\mathrm{mAb}$ & $5 \mu \mathrm{g} / \mathrm{ml}$ & Harlow et al. 1981 \\
\hline SV40 T Ag & $\mathrm{PAb} 419$ (L19) & Biotin mouse mAb & $5 \mu \mathrm{g} / \mathrm{ml}$ & Harlow et al. 1981 \\
\hline SV40 T Ag & PAb 423 (L23) & Biotin mouse mAb & $5 \mu \mathrm{g} / \mathrm{ml}$ & Harlow et al. 1981 \\
\hline
\end{tabular}

Table 2.3 - Antibodies used in immunolabelling studies. The notation for antibodies to SV4O $T A g$ is as given in Crawford and Harlow, 1982. EPOS mAbs were from DAKO (Glostrup, Denmark).

\subsubsection{Labelling tissue culture cells}

Adherent cells were removed with $2 \mathrm{mM}$ EDTA and pelleted. The pellet was rinsed and resuspended in PBS. Cytospins were made by centrifugation onto glass slides at 2,000 rpm for 5 minutes (Cytospin2, Shandon, Runcorn). The cytospins were air-dried, fixed by immersion in acetone for 10 minutes and stored at $-20^{\circ} \mathrm{C}$. 


\subsubsection{Frozen material, fixed after sectioning}

Following sacrifice a piece of tissue up to $0.2 \mathrm{~g}$ in weight was placed in a plastic vial (no. 731, Scientific and Medical Products, Didsbury) in Cryo-M-Bed embedding compound $(\mathrm{BDH}$, Poole) and plunged into liquid nitrogen. The pieces of frozen tissue were stored at $-70^{\circ} \mathrm{C}$. These samples were sectioned in a cryostat (cabinet temperature $-25^{\circ} \mathrm{C}$ ) onto gelatin or silane coated multiwell glass slides. Sections were air-dried overnight and then fixed in acetone for 10 minutes at room temperature. Fixed sections were wrapped in foil and stored at $-20^{\circ} \mathrm{C}$.

\subsubsection{Immersion fixation for light microscopy}

This method was generally used for tissues obtained at post-mortem. Tissues were placed in neutral buffered formalin solution (approx 4\% formaldehyde) for 24 hours, and then embedded in paraffin for sectioning by standard techniques. In order to label SV40 T antigen ( $\mathrm{T} \mathrm{Ag}$ ) these sections were treated in the same way as perfusion-fixed, paraffinembedded material (see below).

\subsubsection{Immersion fixation for immunoelectron microscopy}

Immediately after sacrifice the liver was sliced finely and placed on a drop of $4 \%$ paraformaldehyde; cubes (approximately $1 \mathrm{~mm}^{3}$ ) were then cut with a razorblade and fixed by immersion in the same fixative for six hours, prior to proceeding as with material obtained by perfusion-fixation. Preservation of antigenicity appeared similar; the sinusoids were significantly harder to distinguish due to the presence of blood.

\subsubsection{Perfusion-fixation}

The perfusion of kidney and liver with fixatives in situ results in much improved structural and ultrastructural preservation. In order to acheive this, the descending aorta was perfused in a retrograde manner with a constant pressure perfusion apparatus.

The animal was anaesthetised and the aorta and inferior vena cava were exposed and clamped with an atraumatic clip below the renal arteries. A flap was elevated in the aorta using iridectomy scissors and a flexible polyethylene cannula inserted and tied in place (internal diameter, $0.58 \mathrm{~mm}$; external diameter, $0.96 \mathrm{~mm}$; Portex, Hythe). Just before initiation of perfusion, a large incision was made in the right renal vein. The clamp was then released and perfusion commenced at a pressure of $110 \mathrm{mmHg}$, with PBS containing $10 \mathrm{u} / \mathrm{ml}$ heparin. The thorax was opened and the aorta and vena cava clamped above the diaphragm. Approximately 30 seconds after starting perfusion, when the kidney was completely 
blanched, the perfusate was changed to fixative. The following fixatives were used; (1) $2.5 \%$ paraformaldehyde with $0.1 \%$ glutaraldehyde (2) $4 \%$ paraformaldehyde (3) PLP (2\% paraformaldehyde/0.075M L-lysine monohydrochloride/0.01M sodium periodate in $0.1 \mathrm{M}$ phosphate buffer) (4) $2 \%$ glutaraldehyde. Fixation was for 15 minutes, followed by a 5 minute flush with buffer. The tissue obtained was then processed in three different ways;

a) half of one kidney was embedded in paraffin by standard methods and $3 \mu \mathrm{m}$ sections cut onto silane-coated slides. Prior to immunostaining sections were dewaxed in xylene (three changes, two minutes each), followed by industrial methylated spirit (two changes, two minutes each), and then rehydration in $70 \%$ ethanol (two minutes) and phosphate buffered saline.

b) half of one kidney was equilibriated in $30 \%$ sucrose in $0.1 \mathrm{M}$ phosphate buffer overnight and then frozen in Cryo-M-Bed medium (BDH, Poole) in liquid nitrogen. Subsequently cryosections were cut from this material as described for the frozen material above.

c) radial segments $1 \mathrm{~mm} \times 1 \mathrm{~mm}$ traversing the cortex and medulla were processed for resin embedding and electron microscopy.

Experiments with the different fixatives and buffers suggested that in terms of structural preservation they were similar, and the most important determinant was related to the adequacy of perfusion, assessed by the immediate degree of blanching of the kidney.

\subsubsection{Preservation of antigenicity in different fixation protocols}

Optimal preservation of antigenicity was obtained in the acetone-fixed sections, and exposure to perfusion-fixation appeared to lead to some reduction in sensitivity for most antigens. However, the majority of antigens could be identified in the frozen-fixed material; PLP appeared to be slightly superior to paraformaldehyde in terms of preserving antigenicity. In contrast, in the paraffin-embedded material only three polyclonal antisera (5'NT, SV40 T antigen, and Factor VIII-related antigen) and one monoclonal antibody (ER-TR7) labelled their respective antigens, and each of these required a procedure to restore antigenicity.

For single-labelling experiments examining SV40 T Ag in kidney and liver at the light microscopic level, paraffin sections were generally employed; antigenicity was restored by microwaves $(0.01 \mathrm{M}$ sodium citrate, 10 minutes at $750 \mathrm{~W})$ or by digestion with subtilisin (Type XXIV protease, Sigma, Poole, UK) $125 \mu \mathrm{g} / \mathrm{ml}$ in PBS at $37^{\circ} \mathrm{C}$ for 15 minutes. Doublelabelling was generally performed on frozen perfusion-fixed sections of the kidney or acetone-fixed frozen sections of the liver. 


\subsubsection{Immunostaining for light microscopy}

\section{Single labelling for SV4O T antigen}

If necessary, sections were pre-incubated with $0.3 \%$ hydrogen peroxide, $0.01 \mathrm{M}$ sodium azide to quench endogenous peroxidase activity. The sections were then incubated with a polyclonal rabbit antiserum to SV40 T Ag or normal rabbit serum (as a control) at 1:1000 dilution for 45 minutes, followed by horseradish peroxidase (HRP)-conjugated swine antirabbit immunoglobulin (Ig). The substrate used was nickel-enhanced 3'3'diaminobenzidine (DAB). Sections were then either mounted without counterstaining, lightly counterstained with haematoxylin, or counterstained with periodic acid-Schiff (PAS).

Monoclonal antibodies (mAbs) were also used to label SV40 T antigen. These were murine, and only gave acceptable results when conjugated to biotin, avoiding the need to use antimouse Ig in the second step which gave a high level of background labelling. Succesfull labelling was obtained with biotinylated mAbs PAb 419 and PAb 423 (a gift from Dr J. Gannon; see Harlow, Crawford et al. 1981). It was not possible to use alkaline phosphatase to label SV40 T antigen; this is a recognised difficulty with some other nuclear antigens.

\section{Single labelling for other antigens}

Prior to double-labelling studies, each antibody was used alone to optimise the labelling conditions and to assess the distribution of the antigen. This was generally performed first using peroxidase-conjugated second antibodies, which gave somewhat superior results to alkaline-phosphatase conjugated reagents. The staining was then optimised for alkalinephosphatase labelling.

For desmin two polyclonal rabbit antisera were assessed, but were not found to provide satisfactory labelling of mouse liver sections. Mouse monoclonals necessitated the use of anti-mouse Ig as a second step, giving a high level of background staining. EPOS-conjugated anti-desmin and anti-cytokeratin (murine mAbs directly conjugated to HRP via a polymer backbone) gave excellent results, but did necessitate the use of peroxidase as a substrate for both SV40 T Ag and desmin.

\section{Double-labelling for SV4O T antigen and cytoplasmic/surface antigens}

In double-labelling experiments the SV40 T Ag was always localised first, using peroxidase. Cytoplasmic or cell-surface antigens were then labelled using alkaline phosphatase (with the exception of desmin and cytokeratin). 
For double staining of kidney sections a biotin-conjugated mAb to SV40 T Ag, PAb 419 (Harlow et al. 1981) was first applied at a dilution of $5 \mu \mathrm{g} / \mathrm{ml}$ after blocking endogenous biotin (using a kit from Vector Laboratories, Peterborough). Endogenous peroxidase was then blocked in 4 parts methanol / 1 part $3 \% \mathrm{H}_{2} \mathrm{O}_{2}$ for 5 minutes. Avidin-conjugated HRP (DAKO) was applied (diluted 1:50), followed by nickel-enhanced DAB. The sections were then labelled for surface antigens using rabbit polyclonal antisera or rat mAbs as shown in Table 2.3. Prior to polyclonal antisera, normal swine serum was applied at a 1:50 dilution for 10 minutes. Rabbit polyclonal antibodies were detected with alkaline phosphataseconjugated swine anti-rabbit immunoglobulin diluted 1:25 (DAKO). Rat mAbs were detected with alkaline phosphatase conjugated sheep anti-rat Ig Fab fragments diluted 1:500 (Boehringer Mannheim). Fast Red was used as substrate for alkaline phosphatase. Between each step in the staining procedures, the sections were washed in Tris buffered saline. Following staining, sections were counterstained lightly with haematoxylin, and photographed using an Olympus Vanox microscope.

Double-labelling of liver sections was performed as follows; they were first treated for ten minutes with $0.3 \%$ hydrogen peroxide/0.01 $\mathrm{M}$ sodium azide in Tris buffered saline (TBS) to block endogenous peroxidase activity. They were then incubated with polyclonal rabbit antiserum to SV40 T Ag (1:1000 in TBS) for 30 minutes, followed by horseradish peroxidase(HRP)-conjugated swine anti-rabbit Ig (DAKO) for 30 minutes. Nickel-enhanced $\mathrm{DAB}$ was used to detect peroxidase, and the second procedure was performed immediately thereafter. For rat mAbs the procedure was identical to that described for kidney sections. For ecto-5'-nucleotidase $\left(5^{\prime} \mathrm{NT}\right)$ the sections were first incubated with normal rabbit serum ( $1: 1000$ in TBS) for 10 minutes to saturate the HRP-conjugated secondary antibody used to label SV40 T Ag, prior to proceeding as above. For desmin and cytokeratin, mouse mAbs directly coupled to HRP via an inert polymer backbone were used (EPOS), followed by DAB.

Appropriate controls (normal rabbit serum for polyclonal antibodies, diluent for rat mAbs and EPOS control reagent for EPOS mAbs) were performed on adjacent sections on the same slide in all experiments.

\subsubsection{Immunoelectron microscopy}

Following the fixation described above, samples were rapidly dehydrated through cold ethanol, which was then replaced by a 1:1 mixture of ethanol:LR White resin (London Resin Co., Basingstoke, UK) for 1 hour, prior to overnight impregnation in pure resin at - 
$20^{\circ} \mathrm{C}$. The samples were then embedded in LR White and the blocks polymerised overnight at $70^{\circ} \mathrm{C}$. $1 \mu \mathrm{m}$ sections were cut and stained with Azure A for light microscopy. Selected areas were then sectioned with a diamond knife and placed on nickel grids for immunostaining. A three-stage staining protocol was found to be optimal; non-specific staining was blocked with $1 \% \mathrm{BSA}$ and $1 \%$ Tween in the staining solutions throughout. Grids were first floated on $25 \mu \mathrm{l}$ drops of rabbit polyclonal antisera (diluted 1:1000) to SV40 T Ag, 5'NT or Toxoplasma gondii (negative control) for two hours. This was followed by goat anti-rabbit Ig (Sigma) diluted 1:50 for 1 hour and finally rabbit anti-goat Ig conjugated to $10 \mathrm{~nm}$ colloidal gold (Bio Cell Ltd, Cardiff, UK) for 30 minutes. The grids were then stained with $2 \%$ uranyl acetate for 6 minutes prior to examination with a Jeol 100Cx electron microscope. 


\section{Evidence for a widespread oxygen-sensing mechanism in mammalian cells}

\subsection{INTRODUCTION}

Expression of the Epo gene in the kidney is induced by anaemia or hypoxia. This response involves an increase in gene transcription. Since oxygen is a fundamental requirement for cellular metabolism, it seems likely that the local level of oxygenation will influence the expression of other genes. Examples of candidate genes would be those involved in cellular metabolism and angiogenesis. The demonstration of a hypoxia-responsive enhancer element lying 3 ' to the Epo gene is an important step towards understanding how expression of the Epo gene is increased in response to anaemia. Epo gene expression is tissue-specific; consequently, an important question is whether the oxygen-sensing mechanism controlling Epo gene expression is a specific property of Epo-producing cells or is also present in other cell-types. The experiments described in this chapter were designed to address this issue.

A range of cells that did not produce Epo were tested for the presence of an oxygen-sensing mechanism capable of influencing gene transcription. The experimental approach used was to transiently transfect cells with the Epo 3' enhancer linked to a heterologous promoter and reporter gene. Following transfection, cells were exposed to $1 \%$ or $21 \%$ oxygen; an increase in reporter transcripts in response to hypoxia would demonstrate the presence of a hypoxia-responsive system. In the event of such a response, further experiments would then be necessary to prove that this effect was mediated by the Epo 3' sequence. It would also be possible to assess the effects of pharmacological substances on transfected cells to determine whether the response was similar to that regulating expression of the Epo gene in hepatoma cells. Evidence is presented which shows that many (possibly all) mamalian cells possess a hypoxic-sensing mechanism which is capable of controlling gene transcription. This system is strikingly similar to that which regulates Epo production in hepatoma cells.

\subsection{METHODS}

\subsubsection{DNA constructs}

The DNA constructs used are shown in Figure 3.1; they were provided by Dr P.Ratcliffe and Dr C.Pugh. In the majority of experiments the reporter gene employed was the human $\alpha_{1}$-globin gene. The test plasmid, pBS1-96 $\alpha$, contained the human $\alpha_{1}$-globin gene with $1.4 \mathrm{~kb}$ of $5^{\prime}$ flanking sequence (a BglII-PpuMI fragment) and the first 96 base pairs (see Appendix 1) of the mouse Epo 3' enhancer (inserted as an Xbal linkered PCR product). 
Transient transfection experiments in hepatoma cells had previously demonstrated 10 -fold induction of transcripts from this plasmid in hypoxia (Pugh et al. 1991). In order to control for transfection efficiency and RNA recovery, a second plasmid was transfected into the cells. This co-transfected control plasmid, FGH, contained $290 \mathrm{bp}$ of the promoter of the mouse ferritin heavy-subunit gene joined to the human growth hormone gene. To demonstrate that responses to hypoxia were mediated via the Epo sequence, a second test plasmid, pBS $\alpha$, was used in parallel experiments. This plasmid was identical to pBS1-96 $\alpha$ except that it did not contain the 96 base pairs of Epo sequence.

A
pBS1-96 $\alpha$

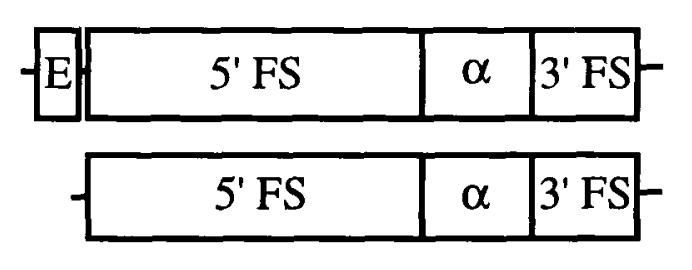
$\mathrm{pBS} \alpha$
96-1-96 SVGH

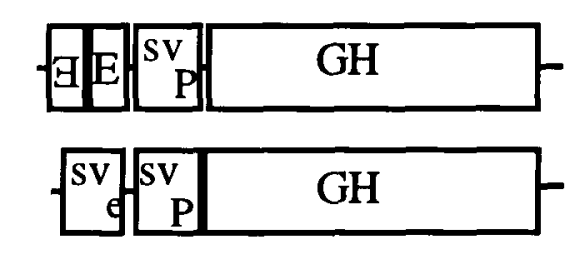
SVeSVGH

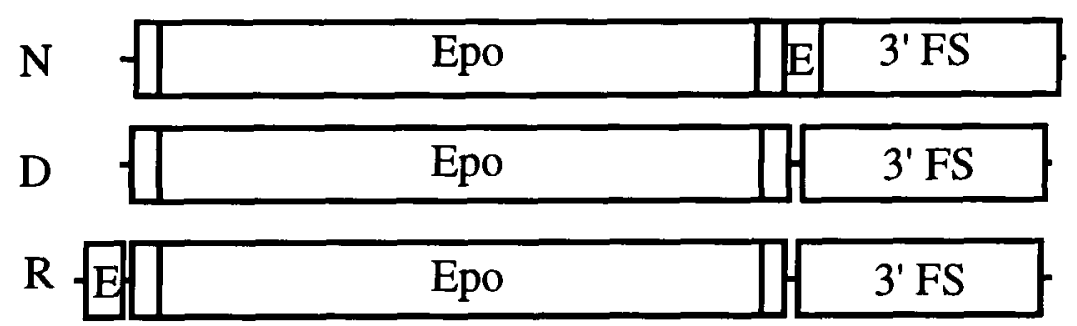

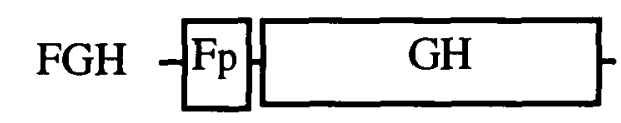

B

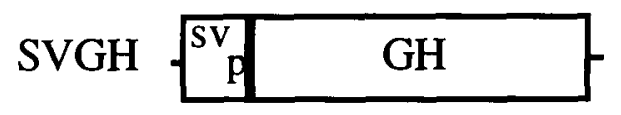

C

Figure 3.1 - A. Plasmids used to test for hypoxic induction via the Epo 3' enhancer. The two test plasmids, pBS $\alpha$ and pBS1-96 $\alpha$, were identical except that pBS1-96 $\alpha$ included the Epo 3' enhancer $(E)$. The enhancer sequence consisted of 96 base pairs (bp) of DNA starting at an Apal site approximately $120 \mathrm{bp} \mathrm{3'}$ to the poly A addition site of the mouse Epo gene and was cloned into polylinker $1.5 \mathrm{~kb} 5$ ' to the $\alpha_{1}$-globin promoter. The $\alpha_{1}$-globin gene was contained in a 2571 bp Bgl2/PpuMI restriction fragment which included 1.4 kilobases $(\mathrm{kb})$ of 5 ' flanking sequence (FS) and $0.5 \mathrm{~kb}$ of $3^{\prime}$ sequence. The co-transfected control plasmid (FGH) contained $290 \mathrm{bp}$ of ferritin promoter $(F p)$ fused to the human growth hormone gene $(G H)$. $B$. Plasmids used to compare the effect of hypoxia on the SV4O enhancer with that on the Epo $3^{\prime}$ enhancer. In all three plasmids, $135 \mathrm{bp}$ of the $S V 40$ promoter $\left(S V_{p}\right)$ was linked to the human growth hormone reporter gene. 96-1-96 SVGH contained two copies of the Epo 3' enhancer in opposite orientations ( $E)$, and $S V S V G H$ contained 240 bp of the $S V 40$ enhancer $(S V)$ ).

$C$. Plasmid ' $N$ ' contained the mouse Epo gene as a $6.1 \mathrm{~kb} \mathrm{Xbal} / \mathrm{Ncol}$ restriction fragment. In ' $D$ ' an Apal/Pvu2 fragment was removed, which included the 3 ' enhancer $(E)$. In ' $R$ ', 96 base pairs containing the enhancer was inserted 5' to the gene at the Xbal site.

Three further plasmids were used to compare the effect of hypoxia on the SV40 enhancer with that on the Epo 3' enhancer (Figure 3.1B). In these three plasmids $135 \mathrm{bp}$ of the SV40 promoter was linked to the human growth hormone gene. 96-1-96 SVGH contained two copies of the 96 bp Epo 3' enhancer in opposite orientations, and SV $\mathrm{e}_{\mathrm{SVGH}}$ contained 240 bp of the SV40 enhancer. In these experiments pBS $\alpha$ was used as the co-transfected control plasmid. 
In order to investigate the operation of the Epo 3' enhancer on the Epo promoter three further plasmids were used which were based on a $6.1 \mathrm{~kb}$ Xbal-Ncol restriction fragment containing the mouse Epo gene (Figure 3.1C). pBS $\alpha$ was used as the co-transfected control plasmid.

\subsubsection{Transfection conditions}

DNA concentrations used for transfection and the transfection voltage were determined in preliminary experiments. For pBS $1-96 \alpha$ or $\mathrm{pBS} \alpha 56 \mu \mathrm{g} / \mathrm{ml}$ was used with $10-20 \mu \mathrm{g} / \mathrm{ml}$ of FGH (depending on the cell line). For the SVGH constructs, $50 \mu \mathrm{g} / \mathrm{ml}$ with $10 \mu \mathrm{g} / \mathrm{ml} \mathrm{pBS} \alpha$ was used. For the Epo constructs, $200 \mu \mathrm{g} / \mathrm{ml}$ was used with $10 \mu \mathrm{g} / \mathrm{ml} \mathrm{pBS} \alpha$.

\subsubsection{Experimental incubation conditions}

Following electroporation, the cells were divided into aliquots. These aliquots were then subjected to normoxic or hypoxic conditions in parallel for 16 hours in $100 \mathrm{~mm}$ Petri dishes containing $8 \mathrm{ml}$ culture medium. In pharmacological studies, the following were added to the medium immediately after electroporation: cobaltous chloride (25-200 $\mu \mathrm{M})$, cycloheximide $(8-200 \mu \mathrm{M})$, and potassium cyanide $(10-1000 \mu \mathrm{M})$. Heat shock was induced by incubating cells at $42^{\circ} \mathrm{C}$, in a normoxic atmosphere in medium supplemented with ${ }^{35} \mathrm{~S}$ methionine (Amersham International) to permit detection of newly synthesised proteins. $72 \mathrm{kDa}$ heat shock protein (Hsp 72) was detected by Western blotting using a mouse monoclonal antibody (RPN1197, Amersham International) and immunoperoxidase.

\subsubsection{RNA analysis}

In most assays 3 to $15 \mu \mathrm{g}$ RNA was subjected to double hybridisation with riboprobes from

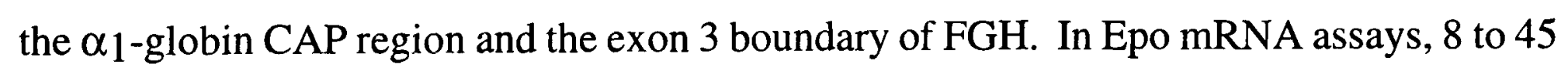
$\mu \mathrm{g}$ RNA was used and the expression of the co-transfected $\alpha_{1}$-globin plasmid was analysed separately on a $3 \mu \mathrm{g}$ aliquot of RNA. For assessment of endogenous Epo expression $100 \mu \mathrm{g}$ RNA from untransfected cells incubated in hypoxia was assayed with complementary riboprobes for man, rat and mouse. For the chinese hamster a riboprobe was constructed using PCR and primers to exons 4 and 5 of the mouse gene (the sequence of the chinese hamster Epo gene and species comparisons are shown in Appendix 3). Results have generally been expressed as a ratio of hypoxic to normoxic expression of the reporter gene, corrected for expression of the transfection control. 


\subsection{RESULTS}

\subsubsection{Initial experiments to determine transfection parameters}

Initial transfections were performed with the DNA constructs shown in Figure 3.1 A. Candidate cell lines were selected to cover a range of mammalian species (from mouse to man), a range of cell types (endothelial, epithelial, macrophage and interstitial) and a range of different organs as shown in Table 3.1. The promoters and reporter genes were selected because they were known to be expressed in a wide range of cells following transient transfection.

\begin{tabular}{|l|l|l|l|}
\hline \multicolumn{1}{|c|}{ Cell line } & \multicolumn{1}{c|}{ Species } & \multicolumn{1}{c|}{ Organ } & \multicolumn{1}{c|}{ Cell type } \\
\hline HepG2 & human & liver & hepatoma \\
\hline Hep3B & human & liver & hepatoma \\
\hline U937 & human & pleural effusion & monocyte \\
\hline MRC5 & human & lung & fibroblast \\
\hline 1BR3 & human & skin & fibroblast \\
\hline GN4 & human & skin & fibroblast \\
\hline COS7 & monkey & kidney & fibroblast \\
\hline BACE & bovine & aorta & endothelial \\
\hline LLC-PK1 & pig & kidney & tubular epithelium \\
\hline FAZA & rat & liver & hepatoma \\
\hline NRK-49F & rat & kidney & fibroblast \\
\hline NRK-52E & rat & kidney & epithelial \\
\hline RAG & rat & kidney & adenocarcinoma \\
\hline RAEC & rat & aorta & endothelial \\
\hline a23 & c hamster & lung & fibroblast \\
\hline K1 & c hamster & ovary & epithelial \\
\hline MEL & mouse & - & erythroleukemia \\
\hline J774.2 & mouse & - & monocyte/macrophage \\
\hline WOP & mouse & skin & fibroblast \\
\hline
\end{tabular}

Table 3.I - Cell lines screened for oxygen-sensing by transfection with constructs containing the Epo 3' enhancer, and susequent hypoxic exposure.

Initial experiments were performed to test whether these constructs were expressed and to optimise transfection voltage. Cultured cells were pooled and divided into four aliquots. These aliquots were transfected with pBS1-96 $\alpha$ and FGH at 300V, 350V, $400 \mathrm{~V}$ and $450 \mathrm{~V}$. Each transfected pool of cells was then divided for normoxic or hypoxic $\left(1 \% \mathrm{O}_{2}\right)$ incubation. The results for such an experiment on MRC5 cells are shown in Figure 3.2 and Table 3.2. The most important observation is that expression of the $\alpha_{1}$-globin reporter was 3.5 to 10 fold higher in hypoxia than in normoxia. A number of other points are illustrated by this data. Firstly, both plasmids are expressed at a quantifiable level across the range of transfection voltages employed. Thus the reporter genes are expressed adequately in this cell type. In this instance $400 \mathrm{~V}$ was selected for further experiments on this cell line. Secondly, as transfection voltage was increased a progressive increase in expression of the test and control plasmids was observed. 


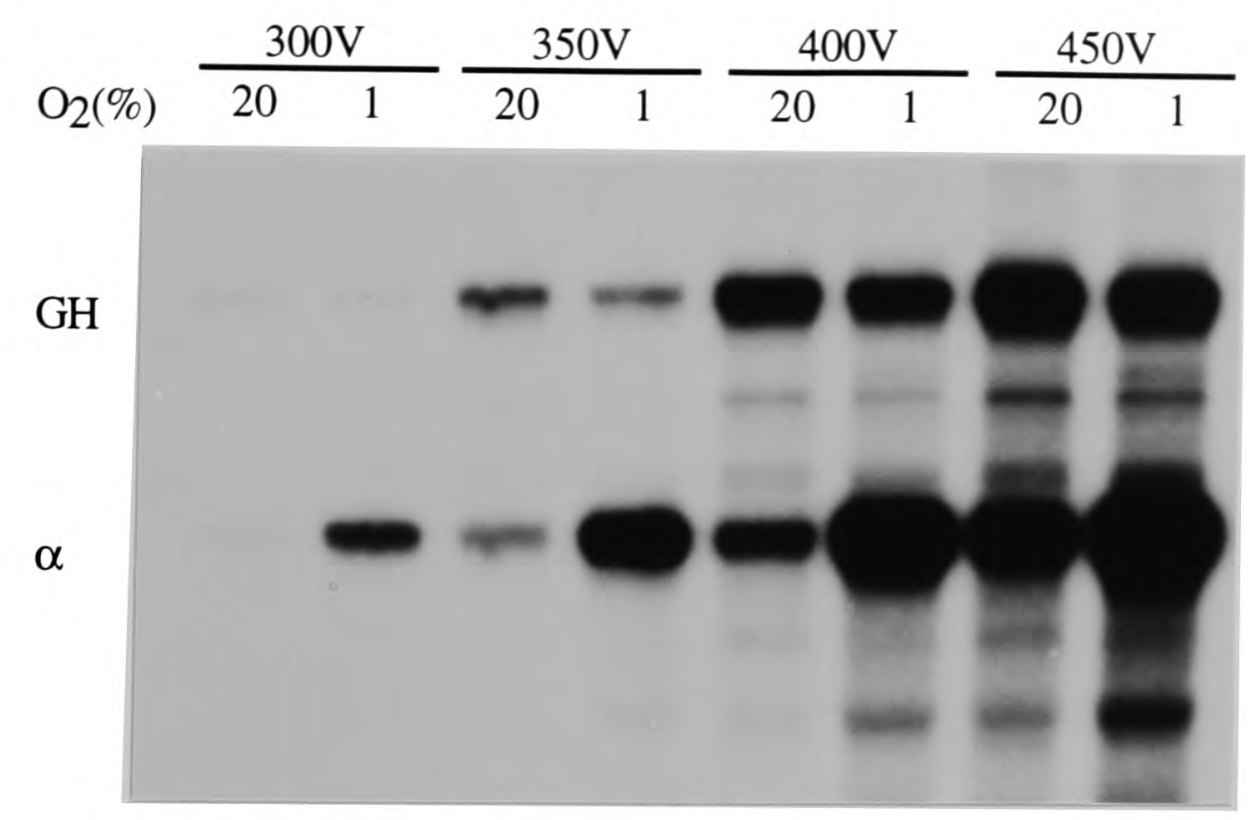

Figure 3.2 - A preliminary experiment with MRC5 cells. A single pool of cells was divided into four aliquots. These were transfected with $\mathrm{PBS1}-96 \alpha$ and FGH at each of the voltages shown and then divided for incubation under normoxic or hypoxic conditions for 16 hours. RNA was hybridised with the $\alpha 98$ and GH probes. Alternate lanes contain RNA from normoxic and hypoxic cells.

\begin{tabular}{|c|c|c|c|c|}
\hline Transfection voltage & $300 \mathrm{~V}$ & $350 \mathrm{~V}$ & $400 \mathrm{~V}$ & $450 \mathrm{~V}$ \\
\hline RNA $(\mu \mathrm{g})$ & 69 & 58 & 29 & 42 \\
\hline$\overline{\alpha(\mathrm{cpm})}$ & $\begin{array}{c}181 \\
1427\end{array}$ & $\begin{array}{c}827 \\
4821\end{array}$ & $\begin{array}{l}2156 \\
13252\end{array}$ & $\begin{array}{r}7999 \\
23034 \\
\end{array}$ \\
\hline$\overline{\mathrm{GH}}(\mathrm{cpm})$ & $\begin{array}{l}212 \\
167\end{array}$ & $\begin{array}{l}1045 \\
781\end{array}$ & $\begin{array}{l}3366 \\
3019\end{array}$ & $\begin{array}{l}6678 \\
5520\end{array}$ \\
\hline $\begin{array}{l}\text { Normoxic } \\
\text { Hypoxic }\end{array}$ & $\begin{array}{l}0.85 \\
8.55 \\
\end{array}$ & $\begin{array}{l}0.79 \\
6.18 \\
\end{array}$ & $\begin{array}{l}0.64 \\
4.39 \\
\end{array}$ & $\begin{array}{r}1.2 \\
4.17 \\
\end{array}$ \\
\hline $\mathrm{H}: \mathrm{N}$ ratio & 10.1 & 7.8 & 6.9 & 3.5 \\
\hline
\end{tabular}

Table 3.2 - Quantitative data from the experiment illustrated in Figure 3.2. Bands were quantified, and the counts obtained are given. The ratio of reporter gene expression in hypoxia to normoxia was calculated (corrected for the level of growth hormone expression). A ratio of greater than 1 indicates hypoxic induction. The amount of RNA recovered is also indicated.

Data from similar experiments with other cells are given in Table 3.3. In this table only the hypoxic to normoxic ratio of $\alpha_{1}$-globin RNA is given (corrected for the transfection control). Three cell lines, U937, MEL and J774.2 cells showed no evidence of hypoxic regulation of reporter gene expression under these conditions. BACE and GN4 cells gave an equivocal result with a ratio of 0.8 to 1.8 . However a two fold or greater induction by hypoxia was observed in the other twelve cell lines. Several cell lines in which a response to hypoxia was observed appear to show a smaller, or no, effect at higher transfection voltage; this is clearly seen in the data for MRC5, 1BR3, FAZA, and RAEC cells. A number of explanations for this phenomenon are possible; for instance reduced cell density in the overnight culture might reduce the effective hypoxic stimulus, or alternatively when there are a high number of transiently transfected DNA molecules in the cells some aspect of the response may become limiting. 


\begin{tabular}{|l|c|c|c|c|}
\hline \multirow{2}{*}{ Cell line } & \multicolumn{4}{|c|}{ Transfection voltage } \\
\cline { 2 - 5 } & $300 \mathrm{~V}$ & $350 \mathrm{~V}$ & $400 \mathrm{~V}$ & $450 \mathrm{~V}$ \\
\hline U937 & & & 0.7 & 0.8 \\
\hline MRC5 & 10.1 & 7.8 & 6.9 & 3.5 \\
\hline 1BR3 & 3.1 & 2.3 & 0.9 & 0.6 \\
\hline GN4 & 1.5 & 1.2 & 1.4 & 0.6 \\
\hline BACE & 1.4 & - & 1.8 & 0.8 \\
\hline LLC-PK1 & 7.9 & 7.6 & 8.0 & 7.2 \\
\hline FAZA & 5.8 & 4.4 & 3.8 & 3.7 \\
\hline NRK-49F & 2.6 & 2.8 & 1.9 & 1.8 \\
\hline NRK-52E & 3.3 & 2.4 & 3.0 & 2.4 \\
\hline RAG & - & - & 3.6 & 4.7 \\
\hline RAEC & 2.5 & 3.4 & 1.8 & 0.9 \\
\hline a23 & - & - & 5.9 & 4.6 \\
\hline K1 & 6.0 & 8.1 & 7.1 & 5.8 \\
\hline MEL & 1.0 & 1.0 & 0.9 & 1.0 \\
\hline J774.2 & - & - & 0.8 & 1.2 \\
\hline WOP & 4.5 & 4.2 & 3.5 & 3.6 \\
\hline
\end{tabular}

Table 3.3 - Data from preliminary experiments to assess optimal transfection parameters for each cell type. The hypoxic to normoxic ratio of $\alpha_{1}$-globin expression (corrected for $G H$ expression) is given in each case. Ratios greater than 1.0 are seen in most of the cell lines. No evidence of induction was seen in MEL cells, U937 cells or J774.2. The data are equivocal for BACE and GN4.

The data given show a clear difference in the relative expression of the two plasmids in hypoxia and normoxia in the majority of cell lines. Next, it was necessary to demonstrate that this hypoxic inducibility was mediated by the Epo 3' enhancer. Further experiments were performed to investigate this in selected cell types and to confirm the preliminary findings. $400 \mathrm{~V}$ was selected for transfection in all cell lines except for 1BR3, for which $325 \mathrm{~V}$ was used. Further experiments were not performed on some of the original cell lines for a variety of reasons. GN4 were not used because the doubling time was found to be low and the RNA yields were poor; BACE cells were transfected with very low efficiency; FAZA is a hepatoma cell line and two human hepatoma cell lines known to produce Epo were included (HepG2 and Hep3B); WOP cells were found to stop adhering soon after reaching confluence. Cos7 cells were included for which the optimal transfection voltage had already been determined as $375 \mathrm{~V}$.

\subsubsection{Hypoxic induction is dependent on the presence of the Epo 3' enhancer}

In order to distinguish action of the Epo 3' enhancer from any other effect of hypoxia on transcript accumulation, transfections using plasmids with (pBS1-96 $\alpha$ ) or without (pBS $\alpha$ ) the Epo 3' enhancer were performed on aliquots of cells from the same pool. Figure 3.3 shows examples of individual transfection experiments for the cell lines Hep3B, U937, MRC5, and 1BR3. This figure also shows that under normoxic conditions the Epo 3' enhancer

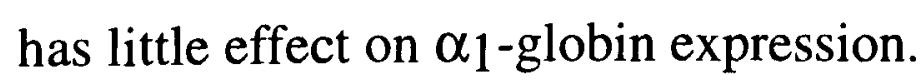




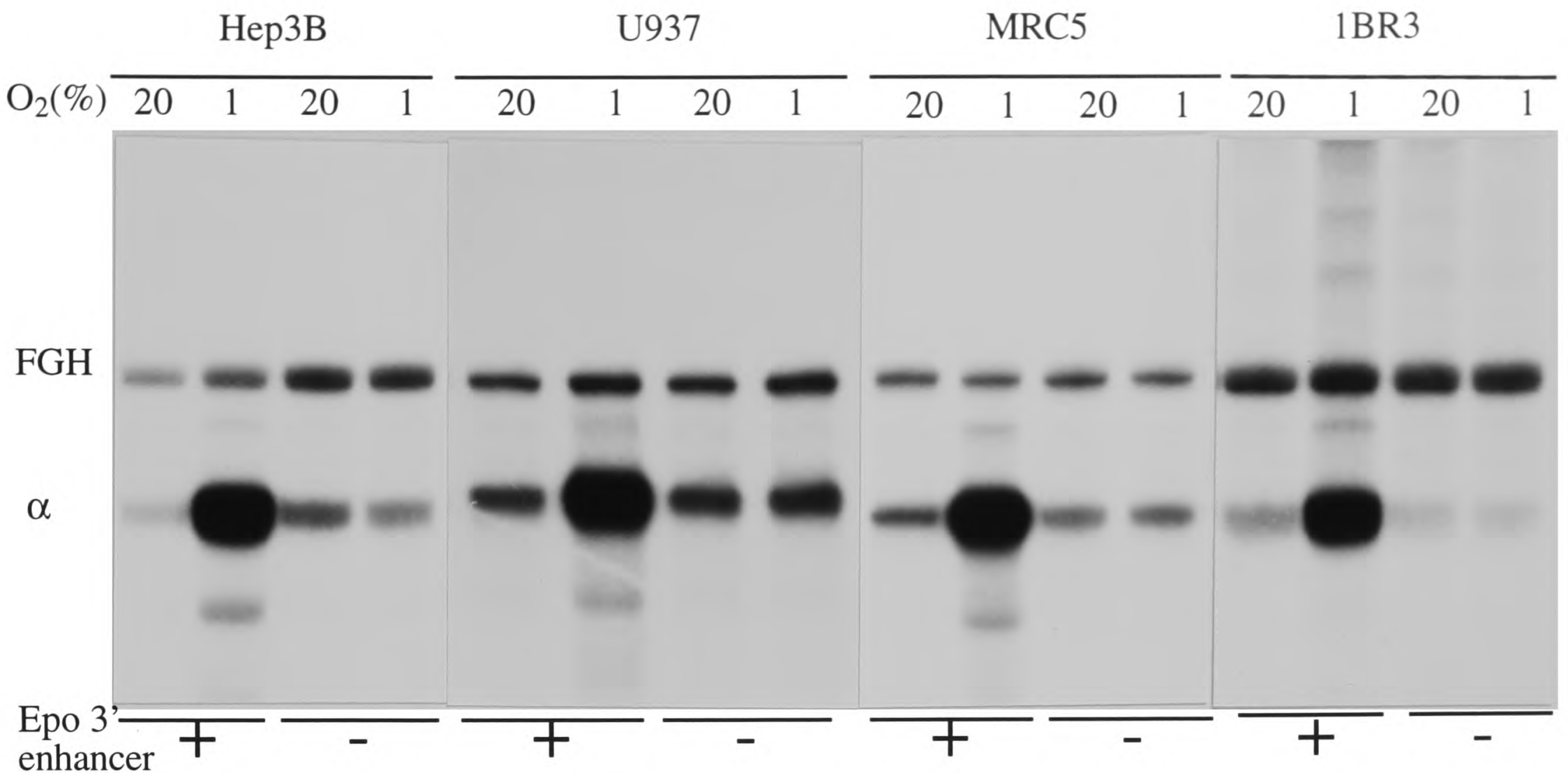

Figure 3.3 - RNase protection assays of reporter $(\alpha)$ and control $(F G H)$ transcripts from transiently transfected human cell lines Hep3B (hepatoma), U937 (monocyte/macrophage), $M R C 5$ (lung fibroblast) and 1BR3 (skin fibroblast). Alternate lanes are from parallel normoxic and hypoxic incubations. In each panel the first pair of lanes shows the result when the test plasmid contained the Epo 3' enhancer coupled to the reporter gene (pBS1-96 $\alpha$ ). The enhancer was absent in the second pair of lanes ( $p B S \alpha)$. Induction of enhancer activity by hypoxia is observed in all these cell lines.

Experiments on all cell lines are summarised in Table 3.4. Hypoxic induction via the Epo 3' enhancer was demonstrated in nine of the ten non-hepatoma cell lines. The three to ten fold induction in $1 \%$ oxygen was similar to that observed in the Epo-producing hepatoma cell lines Hep3B and HepG2. 


\begin{tabular}{|c|c|c|c|c|}
\hline Cell & Species \& type & $\mathrm{O}_{2}$ & \multicolumn{2}{|c|}{ Hypoxic induction } \\
\hline Hep 3B & $\begin{array}{l}\text { Human } \\
\text { Hepatoma }\end{array}$ & $\begin{array}{l}1 \% \\
1 \%\end{array}$ & $\begin{array}{l}9.4 \\
6.9\end{array}$ & $\begin{array}{l}(0.9) \\
(1.0)\end{array}$ \\
\hline Hep G2 & $\begin{array}{l}\text { Human } \\
\text { Hepatoma }\end{array}$ & $\begin{array}{c}1 \% \\
0.1 \%\end{array}$ & $\begin{array}{l}8.2 \\
8.8\end{array}$ & $\begin{array}{l}(1.0) \\
(1.5)\end{array}$ \\
\hline MRC5 & $\begin{array}{l}\text { Human } \\
\text { Foetal lung }\end{array}$ & $\begin{array}{l}1 \% \\
1 \% \\
1 \% \\
1 \%\end{array}$ & $\begin{array}{l}5.2 \\
4.5 \\
8.7 \\
9.5\end{array}$ & $\begin{array}{l}(0.7) \\
(0.7) \\
(1.3) \\
(1.2)\end{array}$ \\
\hline 1BR3 & $\begin{array}{l}\text { Human } \\
\text { Skin fibroblast }\end{array}$ & $\begin{array}{l}1 \% \\
1 \% \\
1 \% \\
1 \%\end{array}$ & $\begin{array}{l}5.8 \\
7.9 \\
5.8 \\
3.2\end{array}$ & $\begin{array}{l}(0.9) \\
(1.0) \\
(1.0) \\
(0.9)\end{array}$ \\
\hline $\cos 7$ & $\begin{array}{l}\text { Monkey } \\
\text { Renal fibroblast }\end{array}$ & $\begin{array}{c}1 \% \\
1 \% \\
1 \% \\
0.5 \%\end{array}$ & $\begin{array}{c}5.4 \\
6.5 \\
4.1 \\
10.8\end{array}$ & $\begin{array}{l}(1.8) \\
(1.6) \\
(1.6) \\
(1.7)\end{array}$ \\
\hline LLC-PK1 & $\begin{array}{l}\text { Pig } \\
\text { Renal epithelial }\end{array}$ & $\begin{array}{c}1 \% \\
1 \% \\
1 \% \\
0.1 \% \\
\end{array}$ & $\begin{array}{l}3.7 \\
8.3 \\
6.4 \\
6.7 \\
\end{array}$ & $\begin{array}{l}(0.6) \\
(1.2) \\
(1.4) \\
(1.0) \\
\end{array}$ \\
\hline RAEC & $\begin{array}{l}\text { Rat } \\
\text { Aortic endothelial }\end{array}$ & $\begin{array}{c}1 \% \\
1 \% \\
1 \% \\
0.5 \% \\
\end{array}$ & $\begin{array}{l}4.6 \\
7.6 \\
5.1 \\
5.0\end{array}$ & $\begin{array}{l}(1.7) \\
(1.1) \\
(1.8) \\
(1.8)\end{array}$ \\
\hline $\mathrm{a} 23$ & $\begin{array}{l}\text { Chinese hamster } \\
\text { Lung fibroblast }\end{array}$ & $\begin{array}{c}1 \% \\
1 \% \\
1 \% \\
0.5 \%\end{array}$ & $\begin{array}{c}20.9 \\
15.5 \\
7.5 \\
12.6\end{array}$ & $\begin{array}{l}(2.0) \\
(1.4) \\
(1.8) \\
(1.2)\end{array}$ \\
\hline$\overline{\mathrm{K} 1}$ & $\begin{array}{l}\text { Chinese hamster } \\
\text { Ovary }\end{array}$ & $\begin{array}{l}1 \% \\
1 \% \\
1 \% \\
1 \%\end{array}$ & $\begin{array}{l}5.2 \\
5.6 \\
6.5 \\
6.8\end{array}$ & $\begin{array}{l}(1.6) \\
(1.3) \\
(1.7) \\
(2.4)\end{array}$ \\
\hline RAG & $\begin{array}{l}\text { Mouse } \\
\text { Renal adenocarcinoma }\end{array}$ & $\begin{array}{l}1 \% \\
1 \% \\
1 \% \\
1 \% \\
\end{array}$ & $\begin{array}{l}2.8 \\
4.5 \\
3.5 \\
3.8 \\
\end{array}$ & $\begin{array}{l}(0.8) \\
(0.9) \\
(1.0) \\
(0.8) \\
\end{array}$ \\
\hline MEL & $\begin{array}{l}\text { Mouse } \\
\text { Erythroleukemia }\end{array}$ & $\begin{array}{l}3 \% \\
1 \% \\
1 \% \\
1 \% \\
0.1 \% \\
0.1 \% \\
\end{array}$ & $\begin{array}{l}1.4 \\
1.1 \\
1.1 \\
1.1 \\
0.9 \\
1.3\end{array}$ & $\begin{array}{c}(1.1)^{*} \\
(1.1) \\
(1.0) \\
(0.6) * \\
(0.6) \\
(1.1)\end{array}$ \\
\hline
\end{tabular}

Table 3.4 - Each line summarises the results of one experiment. Cells were transfected with the plasmids in Figure 3.1A and incubated in parallel in normoxia and hypoxia. Reporter gene $(\alpha)$ expression was corrected for expression of the co-transfected control gene $(F G H)$. The table shows the ratio of hypoxic to normoxic expression (hypoxic induction) for the plas-

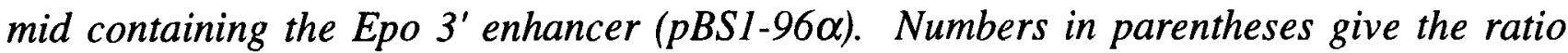
obtained for the parallel transfections with the plasmid which did not contain the Epo $3^{\prime}$ enhancer $(p B S \alpha)$. MEL cells were clone 707; except where indicated ${ }^{*}$, when clone 585 cells were used. Hypoxic induction of reporter gene expression was demonstrated in all cell lines except MEL. The hypoxic response was dependent on the presence of the enhancer.

\subsubsection{Effect of different degrees of hypoxia}

The majority of the experiments were performed at $1 \%$ oxygen. However, in a limited number of experiments different oxygen tensions were employed. Since only one hypoxic incubator was available it was not possible to compare different levels of hypoxia on cells from a single electroporation. More severe hypoxia did not (in most cells) appear to increase induction of enhancer activity. 


\subsubsection{Further experiments with MEL and U937 cells}

The initial experiments on U937 and MEL described above did not demonstrate induction by $1 \%$ oxygen. However, when more severe hypoxia and higher cell densities were tested, U937 cells exhibited a similar level of hypoxia-inducible enhancer activity to the adherent cell lines. Figure 3.4 shows the results of two experiments, one comparing a range of cell densities in U937 cells at $1 \%$ oxygen and the second performed at $0.1 \%$ oxygen.

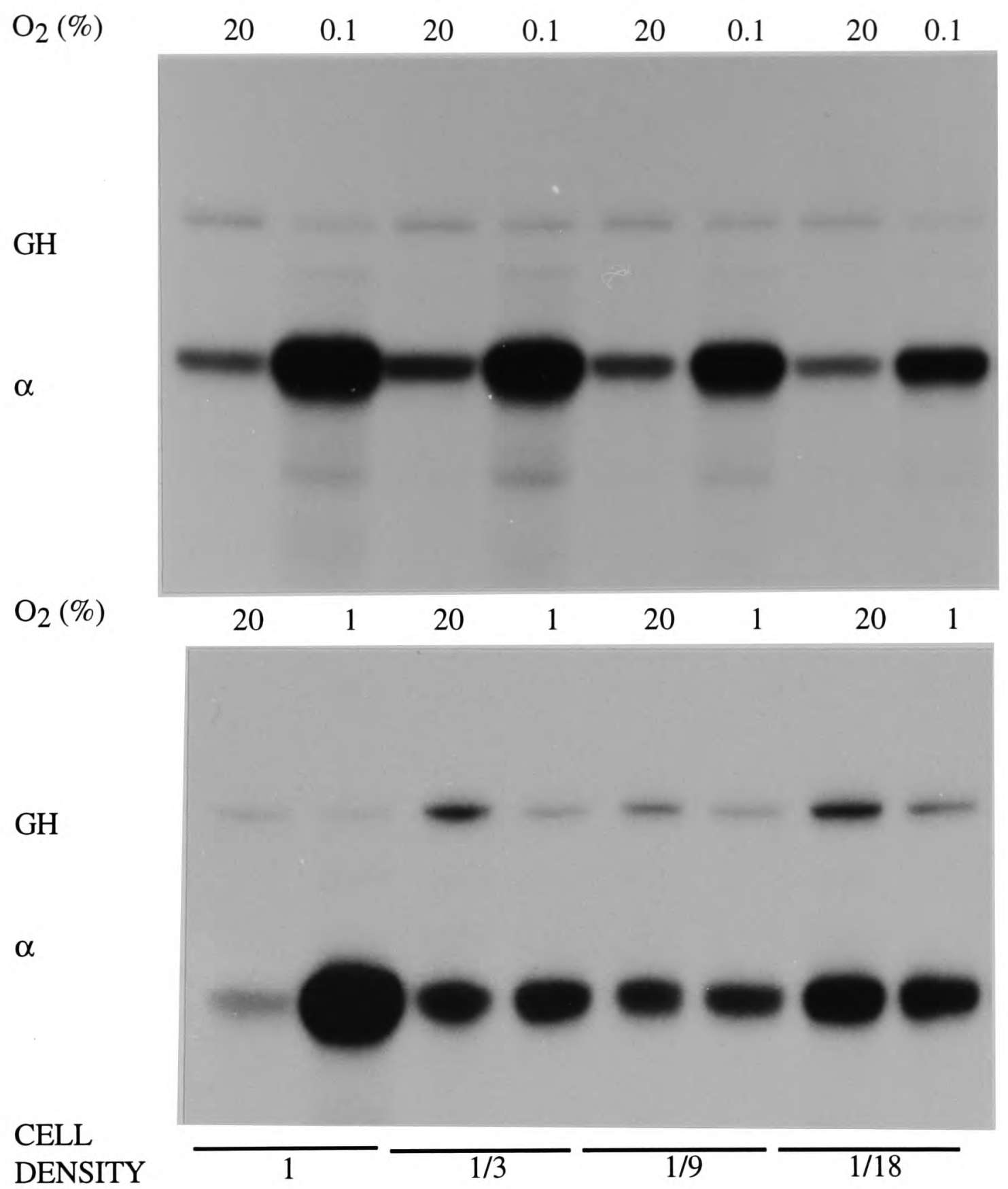

Figure 3.4 - RNase protection assays showing results of two experiments on transfected U937 cells. After transfection the cells were divided for incubation at different densities under normoxic and hypoxic conditions. Alternate lanes contain RNA from the normoxic and hypoxic cells. In the upper panel the hypoxic stimulus was $0.1 \%$ oxygen. In the lower panel the stimulus was $1 \%$ oxygen. Hypoxic induction is seen over the whole range of cell density in $0.1 \%$ oxygen, but only at high cell density in 1\% oxygen. Parallel experiments with cells transfected with $p B S \alpha$ showed no evidence of hypoxic induction of $\alpha_{1}$-globin expression over the same range of cell density.

Table 3.5 summarises the results of all four experiments in which serial dilutions of U937 cells from a single pool were made following transfection. In each experiment, lower cell density reduced the magnitude of the response. In the two experiments at $0.1 \%$ oxygen inducibility was greater than that seen at $1 \%$ oxygen. In contrast, in MEL cells there was little, if any, hypoxia-inducible enhancer activity when studied over a similar range of cell 
densities at $0.1 \%$ and $1 \%$ oxygen, as shown in Table 3.6. In addition no effect was seen at $3 \%$ oxygen. Since it was possible that the failure to demonstrate oxygen-sensing was a feature of this particular clone (707) of MEL cells, a second clone (585) was also tested with similar results. MEL cells were thus the only cell type tested in detail which did not show substantial hypoxic induction. When the results of all the experiments performed with Clone 707 cells and $1 \%$ oxygen were analysed there was no significant difference between hypoxic induction with and without the Epo 3' enhancer, and the values were very close to $1.0(1.09+/-0.11$ vs $1.00+/-0.10$, mean+/-sd, $n=5)$. However, at $0.1 \%$ oxygen there was a small, but statistically significant difference $(1.31+/-0.03$ vs $0.83+/-0.03$, mean+/-sd, $\mathrm{n}=10 ; \mathrm{p}<0.001$, paired $\mathrm{t}$ test).

\begin{tabular}{|c|c|c|c|c|c|}
\hline Index density & $\mathrm{O}_{2}$ & \multicolumn{4}{|c|}{ Hypoxic induction } \\
$\times 0^{-} 6 / \mathrm{ml}$ & \multirow{2}{*}{ Dilution of index density } \\
\cline { 3 - 6 } & & - & $1 / 3$ & $1 / 9$ & $1 / 18$ \\
\hline 5.7 & 1 & $4.7(0.8)$ & $3.0(0.9)$ & $1.5(1.0)$ & $1.4(0.9)$ \\
\hline 2.2 & 1 & $1.3(0.8)$ & $1.3(0.6)$ & $1.2(0.8)$ & $1.0(0.7)$ \\
\hline 2.9 & 0.1 & $9.8(0.7)$ & $5.4(0.6)$ & $4.4(0.7)$ & $4.5(0.7)$ \\
\hline 2.3 & 0.1 & $4.7(0.6)$ & $4.3(0.7)$ & $3.0(0.8)$ & $3.0(0.8)$ \\
\hline
\end{tabular}

Table 3.5 - Hypoxic induction in $U 937$ varies with cell density. Four separate experiments are summarised. In each, a single pool of transfected cells was divided for subsequent incubation over an 18 fold range of cell density. The table shows the ratio of hypoxic to normoxic expression (hypoxic induction) for the plasmid containing the Epo 3' enhancer (pBS1-96\%). Numbers in parentheses give the ratio obtained for the parallel transfections with the plasmid which did not contain the Epo 3' enhancer ( $p B S \alpha)$.

\begin{tabular}{|c|c|c|c|c|c|}
\hline \multirow[t]{2}{*}{$\begin{array}{c}\text { Index density } \\
\times 10^{-6} / \mathrm{ml}\end{array}$} & \multirow[t]{2}{*}{$\begin{array}{l}\mathrm{O}_{2} \\
\%\end{array}$} & \multicolumn{4}{|c|}{$\begin{array}{c}\text { Hypoxic induction } \\
\text { Dilution of index density }\end{array}$} \\
\hline & & - & $1 / 3$ & $1 / 9$ & $1 / 18$ \\
\hline 5.7 & $\overline{1}$ & $1.3(0.7)$ & $1.0(0.9)$ & $1.1(1.0)$ & $1.0(0.9)$ \\
\hline 4.3 & 0.1 & $1.2(0.4)$ & $1.3(0.8)$ & $1.5(0.9)$ & $1.2(0.8)$ \\
\hline 2.9 & 0.1 & $1.4(0.7)$ & $1.4(1.0)$ & $1.7(0.9)$ & $1.3(1.1)$ \\
\hline
\end{tabular}

Table 3.6 - Experiments on MEL cells at different density. The data is presented as in Table 3.5 .

\subsubsection{Effect of density in adherent cells}

Thus in the case of U937 cells, which are non-adherent, a marked effect of cell density was demonstrated. The effects of cell density in culture before and after electroporation were also examined in a 23 cells. Cells from a single pool were either grown under high or low density conditions for 48 hours prior to transfection. Following transfection the cells were further divided for high or low density culture (Table 3.7). There was not a consistent effect of culture at higher density prior to electroporation; however there was a moderate increase in hypoxic induction when the cells were cultured at high density after transfection. 


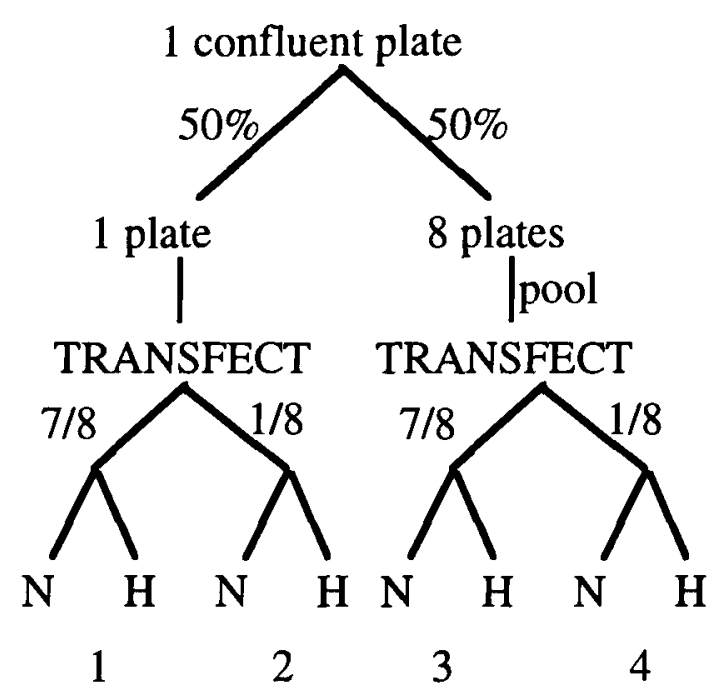

\begin{tabular}{|l|c|c|c|c|}
\hline Pre-electroporation & \multicolumn{2}{|c|}{ HIGH } & \multicolumn{2}{c|}{ LOW } \\
\hline Post-electroporation & High (1) & Low (2) & High (3) & Low (4) \\
\hline H:N ratio & 12.3 & 3.9 & 7.8 & 5.0 \\
\hline
\end{tabular}

Table 3.7 - Effect of cell density on hypoxic induction in a23 cells. Cells, originally from a single pool, were cultured either at high or low density ( 18 fold difference) prior to electroporation. Following electroporation cells were divided for high and low density incubation ( 8 fold difference) under normoxic and hypoxic $\left(1 \% \mathrm{O}_{2}\right)$ conditions. Hypoxic induction is calculated as in the previous tables. In both arms, high density incubation after electroporation resulted in higher level induction (12.3 vs 3.9, and 7.8 vs 5.0).

\subsubsection{Investigation of other promoters}

It was possible that the hypoxic response of the Epo 3' enhancer element would be restricted to interaction with the $\alpha_{1}$-globin promoter. To exclude this, transfections were performed with a construct containing two copies of the Epo 3' enhancer placed immediately adjacent to a 135 bp SV40 promoter element (96-1-96SVGH, see Figure 3.1B). This construct showed twenty-fold and three-fold induction by hypoxia in a23 cells and Cos 7 cells respectively (Table 3.8). In contrast, neither this SV40 promoter alone, the SV40 enhancer or ferritin promoter showed hypoxic inducibility when tested in a 23 or Cos 7 cells. Under hypoxic conditions the two copies of the Epo 3' enhancer conveyed a similar level of transcriptional activation to the SV40 enhancer in both these cell types (data not shown).

\begin{tabular}{|c|c|c|c|c|}
\hline Cell & $96-1-96$ SVGH & SVeSVGH & SVGH & FGH \\
\hline $\mathrm{a} 23$ & 21.3 & 0.8 & 0.6 & 0.3 \\
\hline $\operatorname{Cos} 7$ & 3.0 & 0.6 & 0.9 & 0.9 \\
\hline
\end{tabular}

Table 3.8 - Testing for hypoxic inducibility of three different SV4O promoted growth hormone and FGH constructs in a23 and Cos7 cells. In each case the ratio of hyoxic to normoxic expression is given (corrected for expression of the co-transfected control plasmid).

Similar experiments were performed in MEL cells; the result is shown in Table 3.9. In both these experiments there was an increase in growth hormone mRNA in hypoxia of similar magnitude in the presence of the SV40 enhancer, the Epo 3' enhancer, or with the SV40 promoter alone. The effect seen was therefore not related to the presence of the Epo 3' enhancer. 


\begin{tabular}{|c|c|c|}
\hline 96-1-96SVGH & SVeSVGH & SVGH \\
\hline 2.9 & 3.3 & 2.1 \\
\hline 2.3 & 1.8 & 2.8 \\
\hline
\end{tabular}

Table 3.9 - Results of two different experiments testing for hypoxic inducibility of three different SV40 promoted growth hormone constructs in MEL (clone 585) cells. The hypoxic stimulus was $0.1 \%$ oxygen. The figures are for ratio of $G H$ expression in hypoxia to normoxia (corrected for expression of the transfection control).

\subsubsection{Investigation of other Epo sequence}

Limited experiments were also performed in which Epo sequence other than the 96 bp 3'

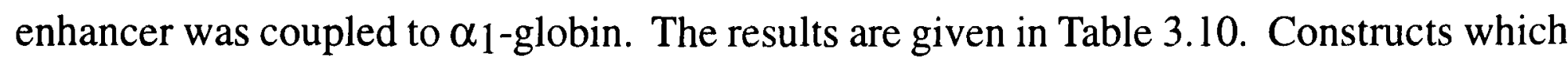
did not include the 3 ' enhancer did not show hypoxic inducibility in transfection studies in hepatoma cells, nor when transfected into other cell lines. The data do suggest that the enhancer was less effective in the context of more extensive Epo sequence (Xbal-Xbal or Xbal-Ncol) and was more effective when two copies were present (96-1-96).

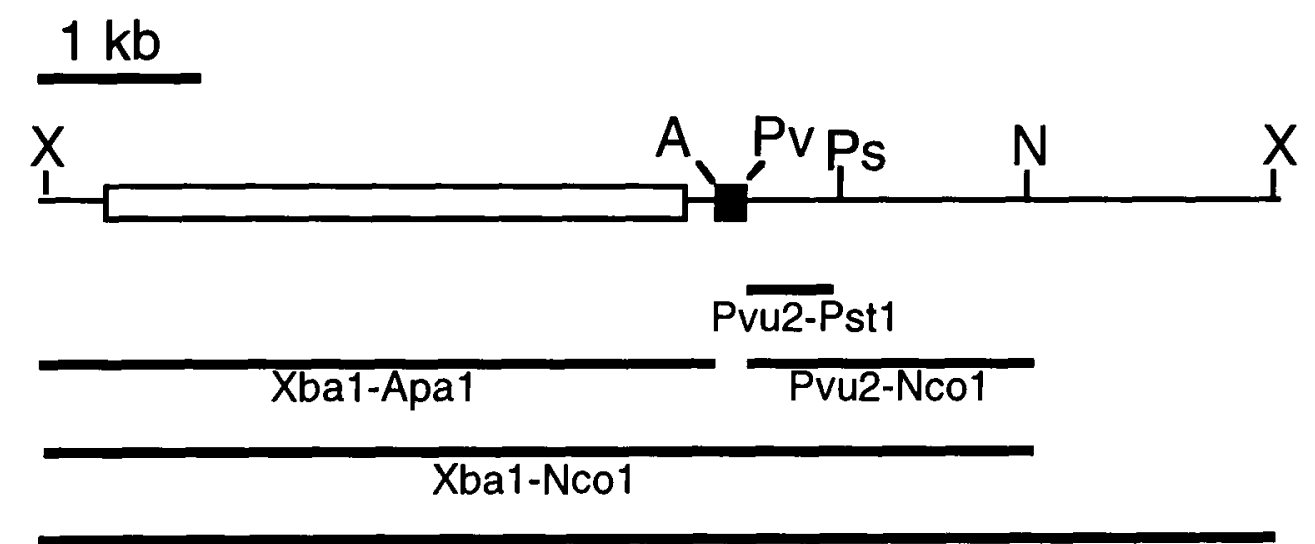

Xba1-Xba1

\begin{tabular}{|l|l|c|l|c|}
\hline Cell & $\begin{array}{c}\text { Constructs with } \\
\text { enhancer }\end{array}$ & H:N ratio & $\begin{array}{l}\text { Constructs without } \\
\text { enhancer }\end{array}$ & H:N ratio \\
\hline a23 & $1-96$ & 14.3 & Xba1-Apa1 & 1.8 \\
& Xba1-Nco1 & 3.3 & Pvu2-Nco1 & 1.3 \\
\hline a23 & $96-1-96$ & 4.6 & Xba1-Apa1 & 1.0 \\
& $1-96$ & 2.5 & Pvu2-Pst1 & 0.5 \\
& Xba1-Xba1 & 1.6 & & \\
\hline FAZA & $1-96$ & 7.3 & Xba1-Apa1 & 1.0 \\
& Xba1-Nco1 & 3.8 & Pvu2-Nco1 & 1.3 \\
\hline U937 & $1-96$ & 1.0 & X-Apa1 & 0.9 \\
& Xba1-Nco1 & 0.9 & Pvu2-Nco1 & 0.8 \\
\hline Co57 & $1-96$ & 2.3 & Pvu2-Pst1 & 1.0 \\
\hline
\end{tabular}

Table 3.10 - Constructs with other Epo sequence linked to $\alpha_{1}$-globin. The data given are from five experiments; in each case the transfections were performed on aliquots from a single pool of cells. The schematic diagram indicates the positions of the restriction fragments. The Epo gene is shown as an open box, the 3' enhancer as a filled box. $X=X b a l, A=A p a l, P v$ $=$ Pvu2, $P$ s $=$ Pst $1, N=$ Ncol .

\subsubsection{Interaction with the Epo promoter}

To examine the interaction of the Epo 3' enhancer with the Epo promoter, the different cell types were transfected with plasmids containing the mouse Epo gene with and without the $3^{\prime}$ enhancer (Figure 3.1C). Transcripts were assayed using a riboprobe which crossed the 
Epo CAP site. As reported previously (Pugh et al. 1991), enhancer action was observed in the hepatoma cell lines Hep3B and HepG2. In both, some incorrectly initiated transcripts were seen, but these were more evident in HepG2. In contrast, in all the other cell types the Epo promoter functioned very weakly; the majority or all of the transcripts (from $70 \%$ to 99\%) being aberrantly initiated. This is illustrated for the $\mathrm{K} 1$ cells and Hep3B cells in Figure 3.5. Enhancer action was consequently difficult to assess but was nevertheless discernible on aberrantly initiated transcripts.

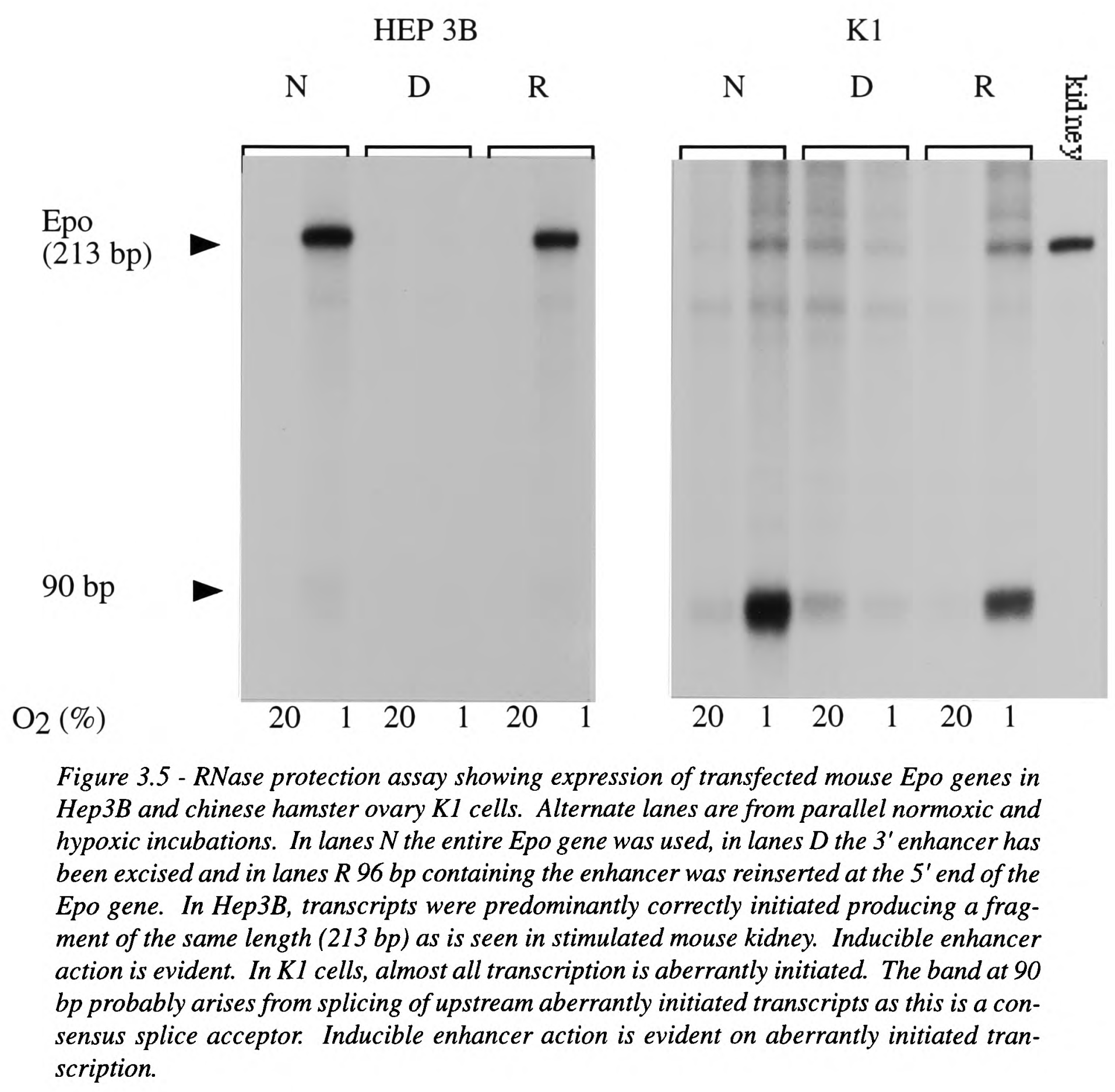

Very weak activity of the Epo promoter and aberrantly initiated transcripts have previously been described by Shoemaker in transfected Cos1 cells (Shoemaker and Mitsock, 1986). Using Northern analysis and S1 nuclease studies he suggested that this was due to the use of a cryptic splice acceptor site at base 535 (Figure 3.6). This explanation would be consistent with the size of the protected fragment (90bp) observed in the current studies. In order to confirm this, primer extension studies were attempted under a range of conditions using RNA from transfected Hep3B cells and four different oligonucleotides shown in Figure 
of a cryptic splice acceptor site at base 535 (Figure 3.6). This explanation would be consistent with the size of the protected fragment $(90 \mathrm{bp})$ observed in the current studies. In order to confirm this, primer extension studies were attempted under a range of conditions using RNA from transfected Hep3B cells and four different oligonucleotides shown in Figure 3.6. These were unsuccessful probably due to the rarity of Epo mRNA; it is possible that poly A selection prior to hybridisation would increase the specificity. This was not pursued since transcripts of this length were never observed in RNA from mouse organs (Chapter 4); it is thus unlikely to be relevant to normal regulation of the gene. Furthermore, the site conforms exactly to a consensus 3' acceptor splice site; (Py)nNPyAGG (Jackson 1991).

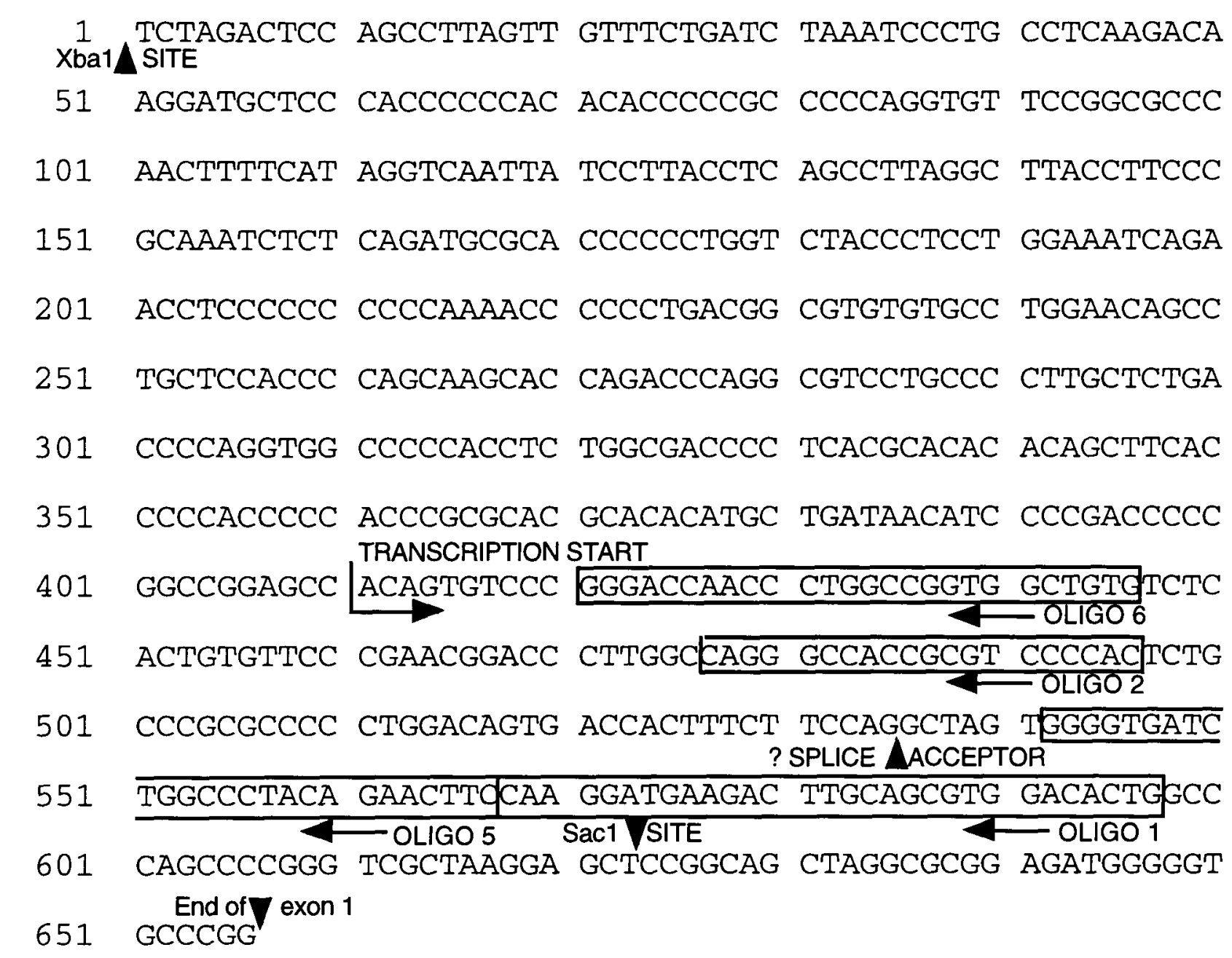

Figure 3.6 - The promoter region and transcription start site of the mouse Epo gene. The four oligonucleotides used in primer extension studies are indicated. The concensus splice acceptor site is also shown.

\subsubsection{Expression of the native Epo gene.}

The cell lines examined were not known to produce Epo. The demonstration of a hypoxiaresponsive system in these cell lines raises three possibilities. Firstly, the cells do in fact produce Epo, and this system is regulating that production. Secondly, this system is regulating other genes. Finally, this system might be present, but not influencing any genes in these cells. 
used; cDNA from hypoxically stimulated chinese hamster kidney and genomic DNA prepared from chinese hamster liver. The riboprobe was hybridised to hypoxic hamster kidney RNA to confirm that it protected a fragment of the correct length. The sequence of the chinese hamster gene in this region is given in Appendix 4.

Expression of the native Epo gene was observed in the hepatoma cells but not in any other cell type. RNA from Cos7 cells (monkey) and LLC-PK1 cells (pig) was hybridised with a human riboprobe. No cross reacting fragments were protected by RNA from $\operatorname{Cos} 7$ but a very low level of hypoxically induced fragments was protected by RNA from LLC-PK1, illustrated in Figure 3.7.

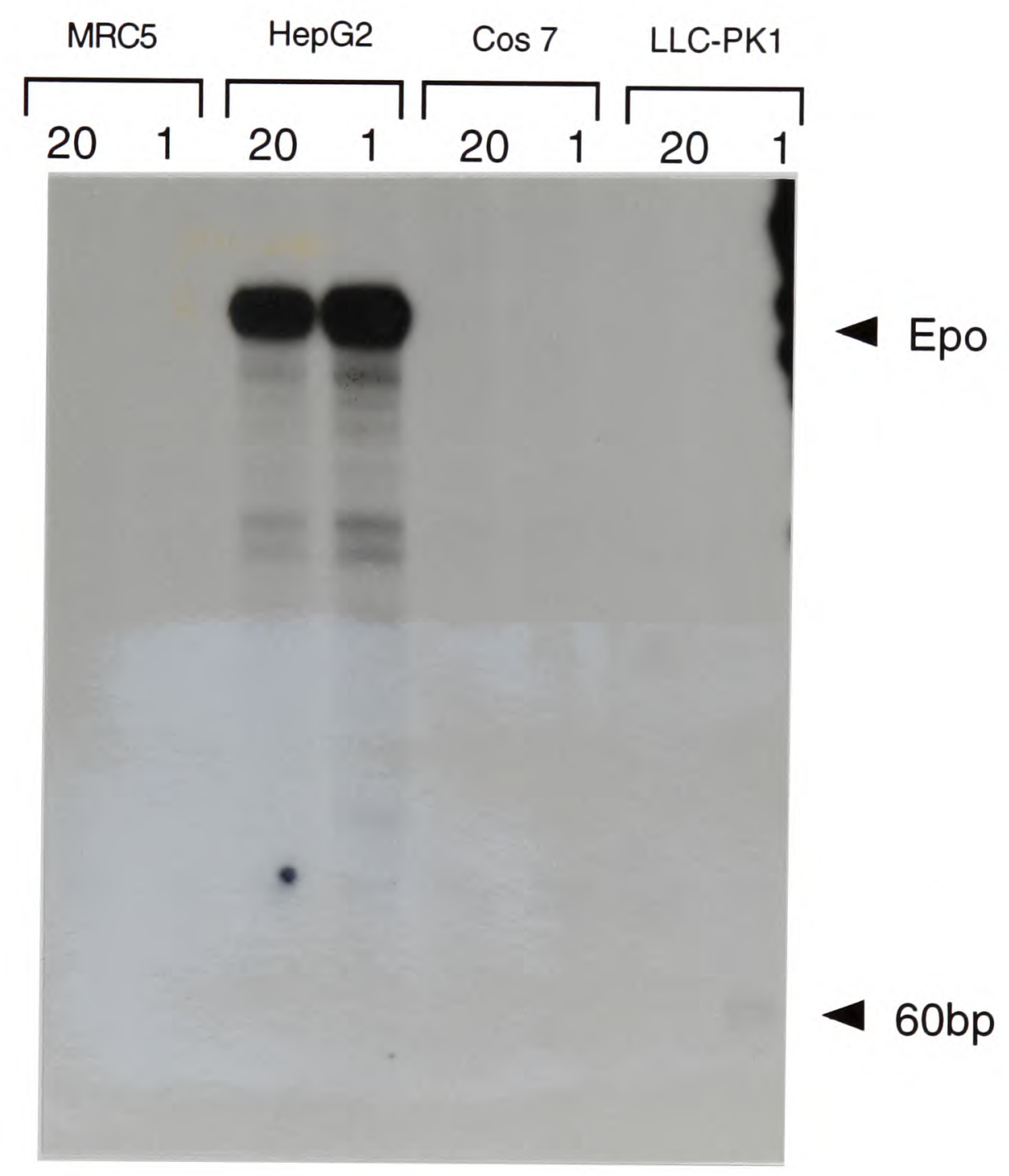

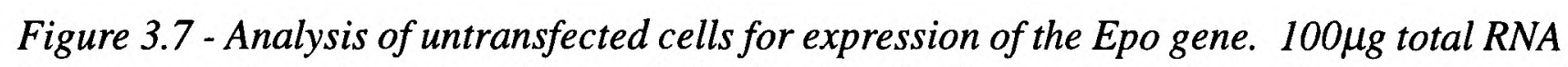
from normoxic or hypoxic cells was hybridised to the human Epo riboprobe. A protected fragment of the predicted length is visible in the lanes containing HepG2 RNA. No protected fragments are seen in the MRC5 cells or IBR3 cells, demonstrating that they do not express Epo at $1 \%$ oxygen. RNA from hypoxic LLC-PKI cells, which are of porcine origin (and hence the riboprobe is not fully complementary) protect a fragment of approximately $60 \mathrm{bp}$. This band is not seen in normoxic LLC-PKI cells. 


\subsubsection{Physiological characteristics of Epo enhancer action in a23 cells.}

Having demonstrated the presence of an oxygen-sensing mechanism which was able to influence expression in diverse cell lines via the Epo $3^{\prime}$ enhancer, experiments were then undertaken to further characterise this response in a non-Epo producing cell line, the chinese hamster lung fibroblastoid line a23 (Westerveld et al. 1971).

Cobalt induces native Epo gene expression both in vivo (Necas and Neuwirt 1971) and in hepatoma cells (Goldberg et al. 1988). In a23 cells, the transfected Epo enhancer was activated approximately ten fold by cobaltous ions in the range $25-200 \mu \mathrm{mol} / \mathrm{l}$ (Figure 3.8). In contrast, but in keeping with physiological regulation of the Epo gene (Necas and Thorling 1972; Tan and Ratcliffe 1991), hypoxic stimulation in a23 cells was not mimicked by exposure to cyanide. Thus cyanide $(10-1000 \mu \mathrm{mol} / \mathrm{l})$ did not induce enhancer activity, nor did it abrogate the response to concurrent hypoxic stimulation (Figure 3.9), although total RNA yield was reduced six fold at the highest dose. The protein synthesis inhibitor cycloheximide blocked induction of enhancer activity by hypoxia in a23 cells, but did not affect unenhanced transcription of the $\alpha_{1}$-globin reporter gene (Table 3.11). This is consistent with the observation that cycloheximide blocks induction of the endogenous Epo gene in hepatoma cells (Goldberg et al. 1988).

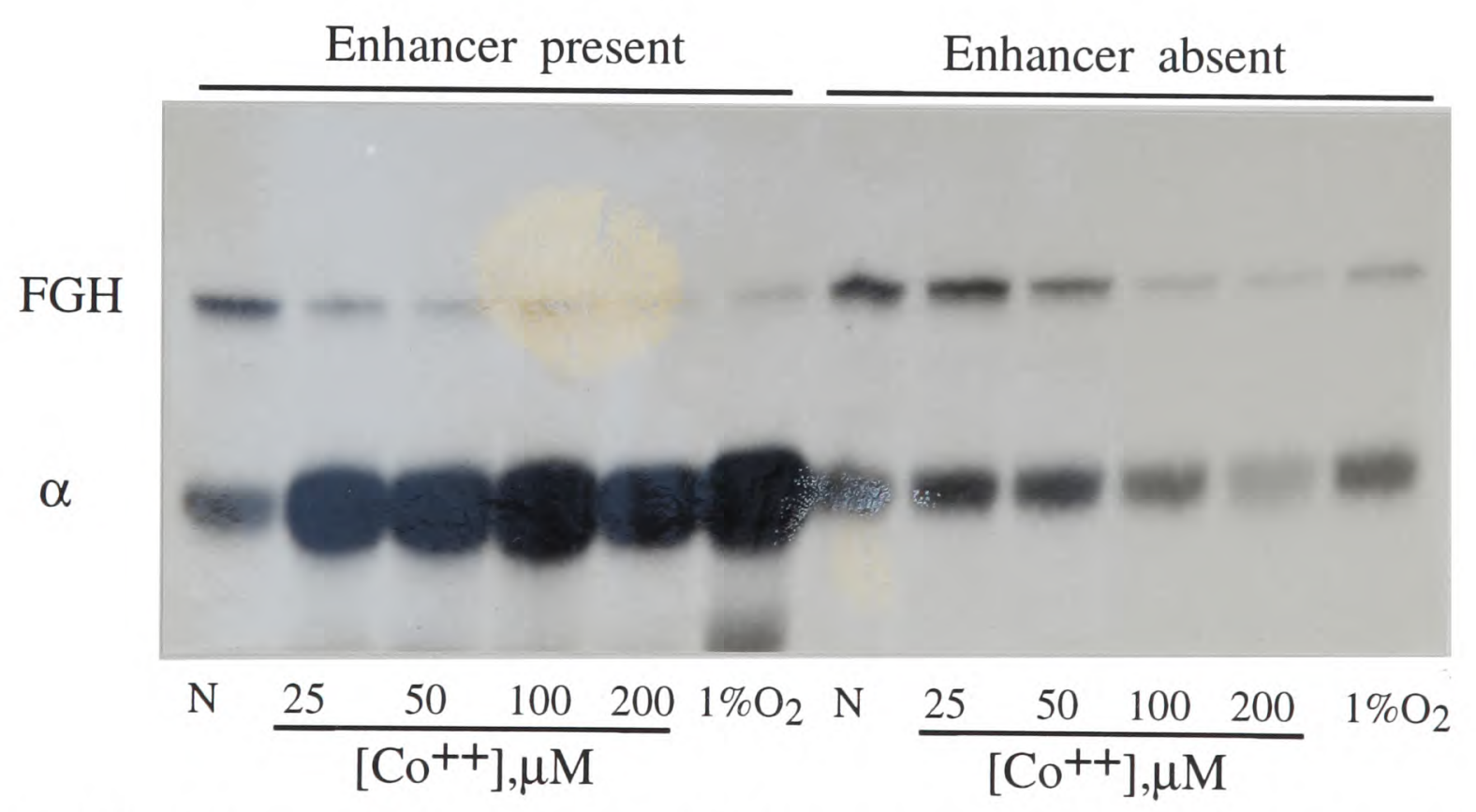

Figure 3.8 - RNase protection assay showing increased expression of the reporter plasmid containing the Epo enhancer in the lung fibroblastoid a23 cells after exposure to cobaltous ions in the range 25-200 $\mu \mathrm{mol}$. The effect is similar to that induced by hypoxia. No response is observed when the reporter plasmid does not contain the Epo enhancer. 


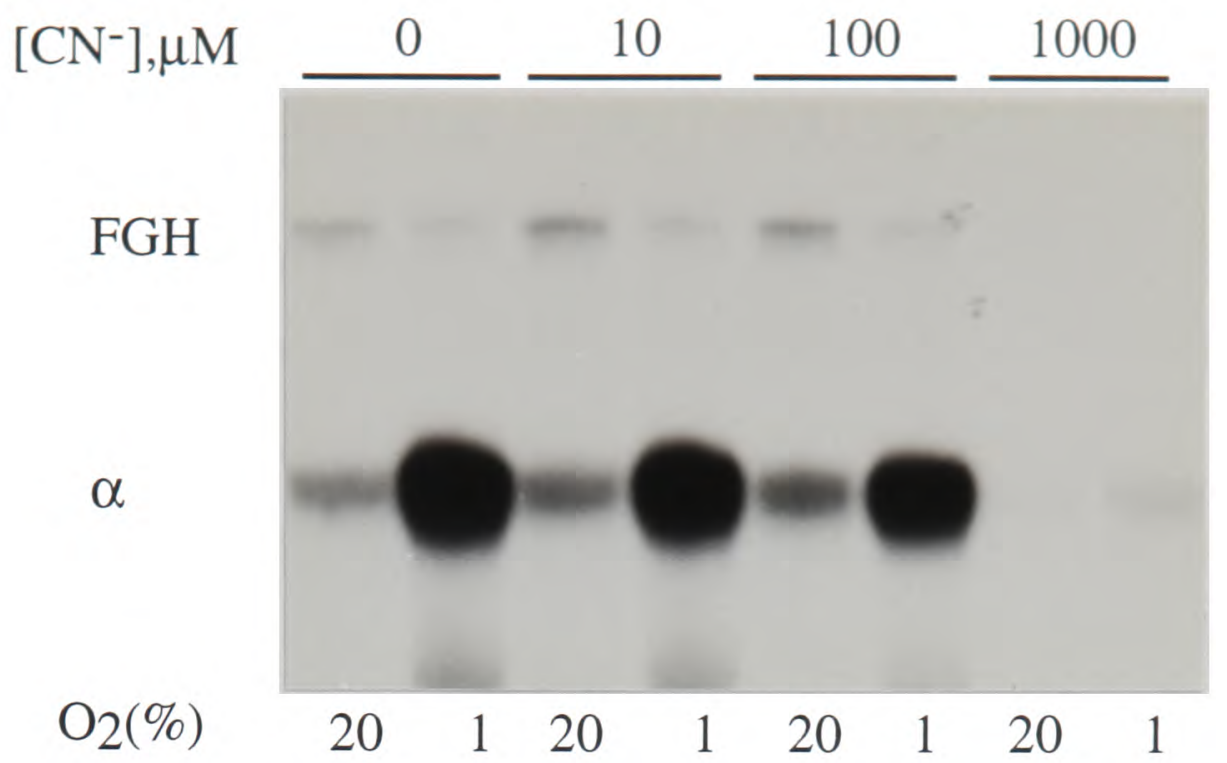

Figure 3.9 - RNAse protection assay showing that exposure to cyanide does not mimic hypoxia in transfected a 23 cells. Alternate lanes are from normoxic and hypoxic incubations in the presence of increasing concentrations of cyanide. In the range 10-1000 $\mu \mathrm{M}$, cyanide did not increase expression of the enhancer-linked gene. Except at the highest concentration, which caused near total cell death, cyanide did not substantially interfere with the response to hypoxia.

\begin{tabular}{|c|c|c|c|c|}
\hline Plasmid & $\begin{array}{c}\text { Control } \\
\mathrm{H}: \mathrm{N}\end{array}$ & $\begin{array}{c}8 \mu \mathrm{M} \mathrm{Cx} \\
\mathrm{H}: \mathrm{N}\end{array}$ & $\begin{array}{c}40 \mu \mathrm{M} \mathrm{Cx} \\
\mathrm{H}: \mathrm{N}\end{array}$ & $\begin{array}{c}200 \mu \mathrm{M} C \mathrm{Cx} \\
\mathrm{H}: \mathrm{N}\end{array}$ \\
\hline $\mathrm{pBS} 1-96 \alpha$ & 15.5 & 2.8 & 1.4 & 1.5 \\
\hline $\mathrm{pBS} \alpha$ & 1.4 & 1.6 & 1.5 & 1.5 \\
\hline
\end{tabular}

Table 3.11 - Cycloheximide blocks the hypoxic response in a23 cells. Transfected cells were incubated in normoxia and hypoxia in the presence of increasing doses of cycloheximide (8$200 \mu \mathrm{M})$. The ratio of hypoxic to normoxic expression (corrected for the transfection control) is given in the presence and absence of the enhancer. At the lowest dose $(8 \mu \mathrm{M})$, cycloheximide substantially reduced hypoxic induction. Normoxic expression was not reduced even at the highest concentration used $(200 \mu M)$.

In addition to these experiments in a23 cells, the effect of cobalt on MEL (clone 585) cells was tested, as an alternative stimulus to hypoxia in these cells. The results are shown in Table 3.12. As with different degrees of hypoxia there was no convincing up-regulation of reporter gene expression.

\begin{tabular}{|c|c|c|c|c|}
\hline \multirow{2}{*}{ Construct } & \multicolumn{4}{|c|}{ Cobalt concentration } \\
\cline { 2 - 5 } & $25 \mu \mathrm{M}$ & $50 \mu \mathrm{M}$ & $100 \mu \mathrm{M}$ & $200 \mu \mathrm{M}$ \\
\hline $\mathrm{pBS} 1-96 \alpha$ & 1.1 & 0.8 & 0.6 & 0.9 \\
\hline $\mathrm{pBS} \alpha$ & 0.8 & 0.7 & 0.5 & 0.7 \\
\hline
\end{tabular}

Table 3.12 - Experiments on MEL (clone 585) cells with cobalt. The value given is the ratio of $\alpha$ globin expression in the cobalt-treated cells to control cells (corrected for expression of the co-transfected FGH plasmid).

\subsubsection{The response is distinct from heat shock}

The widespread presence of this oxygen-sensing system in cultured mammalian cells, and the demonstration that anoxia can induce a heat shock response (Benjamin et al. 1990) raised the possibility that the control mechanism was a part of the heat shock response. The hypoxic response of a 23 cells and the heat shock response were therefore assessed. Heat shock did not induce Epo enhancer activity; thus exposure of a 23 cells to $42^{\circ} \mathrm{C}$ for sixteen 
hours did not increase the level of reporter gene mRNA (Figure 3.10). To be sure that a heat shock response was induced by these conditions, newly synthesised protein was labelled with ${ }^{35} \mathrm{~S}$-methionine and analysed by PAGE. The cells exposed to heat shock showed two discrete bands at molecular weights of approximately $70 \mathrm{kDa}$ and $110 \mathrm{kDa}$. Induction of Hsp72 was proved by Western blotting (Figure 3.10). Conversely, the hypoxic conditions used to induce Epo enhancer activity did not produce a heat shock response. After exposure of a 23 cells to $1 \%$ oxygen for sixteen hours, no changes were detected in labelled proteins by electrophoresis nor was Hsp72 detectable on the Western blot (Figure 3.10).

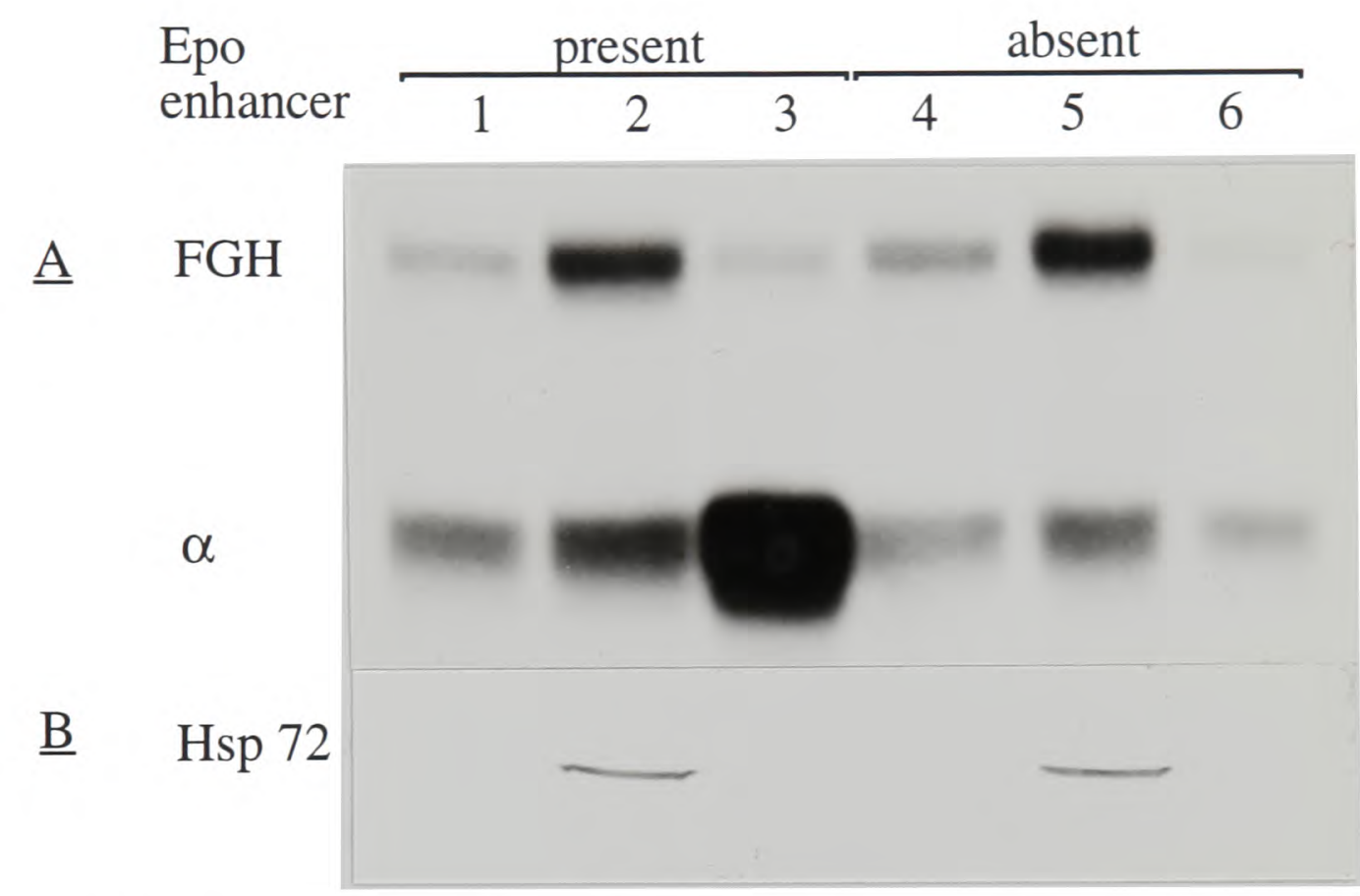

Figure 3.10 - Comparison of the effects of heat shock and hypoxia in a23 cells. (A) RNase protection assay showing that heat shock does not induce a similar response to hypoxia. Transfected cells were incubated in parallel in normoxia at $37^{\circ} \mathrm{C}$ (lanes 1,4$), 42^{\circ} \mathrm{C}$ (lanes 2,5 ) and $1 \%$ oxygen at $37^{\circ} \mathrm{C}$ (lanes 3,6). A slight increase in reporter gene expression is seen in normoxia in the presence of the enhancer. This is similar to that seen in the cells exposed to heat shock. In contrast a large effect of the enhancer is evident in hypoxia.

(B) Western blot showing that $1 \%$ oxygen does not induce Hsp 72. Protein was analysed from aliquots of the transfected cells. Hsp72 was detected only in cells exposed to heat shock (lanes 2 and 5).

\subsection{DISCUSSION}

These experiments were designed to investigate the possibility that, despite tightly restricted expression of the Epo gene itself, the oxygen-sensing mechanism might be widespread, and that tissue-specific restriction of Epo gene expression was achieved by other mechanisms. Cell lines were transiently transfected with recombinant plasmids containing the Epo 3' enhancer coupled to an $\alpha_{1}$-globin reporter gene. This reporter was chosen because it contains a promoter which, under conditions of transient transfection, operates powerfully in multiple cell types and gives rise to a stable mRNA product. That nucleoprotein interactions with transiently transfected DNA allow promiscuous expression of tissue specific genes, such as 
$\alpha$ globin, is well known. However, the demonstration that transfected Epo enhancer activity was inducible by hypoxia strongly suggests that a physiologically relevant component of the oxygen sensing mechanism responsible for regulation of Epo operates widely. That this widespread mechanism can operate via the Epo 3' enhancer indicates that the transcriptional control mechanism shares sequence-specific DNA recognition with the Epo hypoxiaresponsive system.

Further studies on the lung fibroblastoid cell line a23 indicated that in three important respects the response is identical to that governing physiological Epo expression in liver and kidney. Thus it is activated by cobalt but not by cyanide, and is abrogated by cycloheximide. The dose range of cyanide was chosen to include concentrations which cause minimal, partial and total inhibition of cellular respiration (Slater 1967; Tan and Ratcliffe 1991). Oxygen consumption was not measured directly in the current experiments, but the expected toxicity of $1000 \mu \mathrm{M}$ cyanide was apparent from a reduced yield of viable cells and RNA. The lack of effect of cyanide demonstrates that the hypoxic induction of Epo $3^{\prime}$ enhancer action is distinct from the other cellular stresses attendant on the compromise of oxidative metabolism.

Heat shock induction operates widely and anoxia has recently been reported to activate heat shock transcription factor (Benjamin et al. 1990). On the other hand, it is known that heat shock does not induce Epo expression in hepatoma cells (Goldberg et al. 1988). The possibility was tested that the hypoxic response mediated by the Epo 3' enhancer was a manifestation of a heat shock response. The results indicate that this is not the case. Firstly, hypoxia of the severity used did not induce the major heat shock proteins; secondly, heat shock did not activate the Epo enhancer; thirdly, cycloheximide abrogates the Epo enhancer mediated response but is known to induce heat shock mRNA accumulation and thermotolerance (Widelitz et al. 1986). Furthermore, the Epo 3' enhancer sequence does not contain a heat shock element.

Kidney and liver are the major organs producing Epo. However, in rodents low levels of hypoxically inducible Epo mRNA are detectable in some other organs (Tan et al. 1992 and Chapter 4). The cellular source within these organs is not known, so it is difficult to relate this to the current findings. However, since the current experiments demonstrate that oxygensensing is a widespread phenomenon, the hypoxic inducibility of Epo production observed in these organs is not surprising. 
In kidney and liver, Epo expression is limited to a small subset of cells (Koury et al. 1988b; Lacombe et al. 1988; Koury et al. 1991). The range of cells chosen in the current experiments included several which are not known to make Epo and several which are derived from organs which do not contribute importantly to Epo production in vivo (Krantz 1991; Tan et al. 1992). To be sure that these cell lines did not produce Epo, RNA was extracted from hypoxic cells and assayed for Epo mRNA using RNAse protection. Apart from the hepatoma cell lines, no Epo expression was detectable with the possible exception of LLC-PK1. Since a fully complementary probe for LLC-PK1 was not used this finding is not secure but would be consistent with a previous report of low level Epo production by LLC-PK1 (Caro et al. 1984).

Although clear evidence of hypoxia-inducible enhancer activity was observed in eleven of the twelve cell lines tested in detail, activity did vary somewhat between these cell lines. Since the $\mathrm{pO}_{2}$ in the microenvironment of the sensor could bear a different relationship to the incubator $\mathrm{pO}_{2}$ in different cells, it is unlikely that the degree of hypoxia was optimised for enhancer activity in every cell. Thus, apparent differences in inducibility may be intrinsic, or may arise from differences in effective oxygenation. In U937 cells, despite some variability, an interaction between severity of hypoxia and cell density was seen, suggesting that oxygen consumption by respiring cells contributes to cellular hypoxia. Other explanations, such as interaction with cell growth and division, are possible. Increased hypoxic induction at high cell density was also seen in a 23 cells, and may have contributed to the higher inducibility of some cell lines when electroporated at lower voltage in the preliminary experiments. Interestingly, the reverse has been noted for Epo expression in Hep3B and HepG2, where greater induction was observed at lower cell density (Goldberg et al. 1987).

It was difficult to draw a firm negative conclusion about MEL cells from these experiments. However, after extensive study of different conditions using these constructs, it was not possible to show a convincing level of enhancer action in either of two different MEL clones, either in response to hypoxia or to cobalt. Subsequent experiments by other investigators in which a smaller region of the Epo 3 ' enhancer was placed in close proximity to the $\alpha_{1}$ globin promoter have, in fact, demonstrated oxygen-sensing in MEL cells (see below). Thus although hypoxic-sensing was a robust and reproducible phenomenon, it is clear that under certain experimental conditions in particular cell lines this effect would not 
be observed (eg low cell density, particular DNA constructs, high transfection voltage). Such factors probably account for the failure of two previous studies to demonstrate oxygensensing in HeLa cells (Beck et al. 1991) and CHO cells (Pugh et al. 1991).

It is interesting that in transgenic mice (tgEPO4) which included the $3^{\prime}$ enhancer and overexpressed the Epo gene in several tissues hypoxic inducibility was only observed in the liver (Semenza et al. 1989). This is in apparent contrast to the widespread operation of the $3^{\prime}$ enhancer demonstrated in the transfection experiments in this chapter. As discussed in Section 1.4, in the human transgenes the operation of the Epo 3' enhancer will be subject to the influences of other cis-acting elements, the effects of chromatin structure and methylation. Consequently it is not unexpected that an effect seen in these transfection experiments would not be observed in transgenic animals, due to interaction with other mechanisms regulating the Epo gene. An alternative possibility would be that the cells expressing the transgene in other organs in the mice were not sufficiently hypoxic. This is because it is not possible to relate the stimulus used in the transfection experiments $(1 \%$ oxygen in the incubator) to oxygen levels in the region of (as yet) unidentified cells in various mouse tissues.

In the current experiments the Epo 3' enhancer operated widely in the transfected cell lines whereas the Epo promoter did not, being barely active except in the Epo-producing hepatoma cells; this may contribute to the tissue specificity of Epo gene expression in vivo. On the other hand, it is clear from transgenic experiments that tissue-specific expression also requires more distant 5 ' sequence. The operation of tissue-specific gene repression, superimposed on a widespread sensing and signalling mechanism is well established for several responses such as the adenylate cyclase system (Roesler et al. 1988). The widespread existence of this oxygen sensing system presumably indicates that it serves other purposes, rather than solely controlling Epo production.

\subsubsection{Further evidence for a widespread oxygen-sensing mechanism}

Since these experiments were performed further evidence has been produced that confirms the widespread presence of this oxygen-sensing system, and provides important evidence that this oxygen-sensing system controls other genes. Some of this data produced by other workers is discussed. 


\subsubsection{DNA binding activity}

The experiments in this chapter demonstrate a functional system able to act via the Epo 3' enhancer in multiple cell types. Subsequently, a hypoxically-regulated DNA binding activity has been demonstrated in hepatoma cells using electrophoretic mobility shift assays (Wang and Semenza 1993a). This activity, termed HIF-1, was shown to be binding to a region which is completely conserved between mouse and man and corresponds to nucleotides 614 of the 1-96 Epo enhancer element used in the functional studies in this chapter (see Appendix 1). The physiological relevance of this DNA binding activity is supported by several lines of argument. In particular, it is inducible by hypoxia and this induction is blocked by cycloheximide.

An equivalent hypoxically-regulated DNA binding activity in non-Epo producing cells would provide strong supportive evidence for the presence of an oxygen-sensing system in other cells. Wang and Semenza therefore used a labelled oligonucleotide including bases 4-21 of the human Epo 3' enhancer sequence as a probe for hypoxically-inducible binding activity (HIF-1) in a range of cell types (Wang and Semenza 1993c).

The cells studied were derived from chinese hamster ovary ( $\mathrm{CHO})$, human embryonic kidney (293), human cervical carcinoma (HeLa), mouse myoblasts (C2), mouse fibroblasts (Ltk-) and rat fibroblasts (Rat1). The mobility of the complex in non-denaturing gels in each case appeared to be identical to that obtained with hypoxic Hep3B extracts. On denaturing gels following uv cross-linking the species obtained in Ltk, HeLa and CHO co-migrated with that from hepatoma cells. When $\mathrm{CHO}$ cells were treated with cycloheximide, hypoxic induction was prevented, and cobalt was shown to induce the DNA binding activity in CHO cells. Finally, functional studies using transient transfection of Epo sequence linked to SV40 promoted constructs demonstrated hypoxic inducibility in $\mathrm{CHO}$ and 293 cells.

These experiments provide complementary data to that described in this chapter. They provide substantial support for the widespread operation of a hypoxic-response system which bears striking similarities to that controlling Epo expression in hepatoma cells.

\subsubsection{Further functional studies in $M E L$ cells}

The 96 base pairs of Epo sequence used in this chapter were considered likely to contain multiple transcription factor binding sites. Subsequent to the experiments in this chapter, further experiments have been performed in MEL cells (Pugh et al. 1993). The most important finding was that when nucleotides 1-25 were placed close to heterologous promoters, and particularly when reiterated, hypoxic induction was clearly demonstrated in 
MEL cells. It was suggested that the ability to influence the promoter at a distance was dependent on interactions with regions of the enhancer lying 3 ' to these 26 nucleotides. In MEL cells interactions with this region led to suppression of enhancer action, whereas in HepG2 cells the effect was cooperative.

\subsubsection{Functional evidence for this system controling other genes}

There is increasing evidence for a variety of mammalian genes being regulated by hypoxia. Furthermore, in several cases similarities to the system controlling Epo production have been demonstrated.

\section{Growth factors and vasomotor control}

It would be reasonable to predict that the level of environmental oxygen would be an important variable in determining the local blood supply via influences on the rate of production of angiogenic growth factors and vasomotor substances. There is considerable evidence for this. For instance, cultured macrophages have been shown to elaborate an angiogenic growth factor in response to low oxygen tension in culture (Knighton et al. 1983).

There is now evidence for some growth factors and vasomotor substances being regulated in a fashion similar to the Epo gene. In cultured human umbilical vein endothelial cells (HUVEC), expression of the platelet derived growth factor B chain (PDGF-B) is upregulated at $1 \%$ oxygen, and this response was shown to be transcriptional in nature (Kourembanas et al. 1990). PDGF can act as a vasoconstrictor and is also thought to be involved in cellular proliferation and chemotaxis. More recently, PDGF-B induction by hypoxia has been shown in HepG2 and Hep3B cells (Gleadle et al. 1995). The response could also be induced by transition metals and iron chelators, and thus showed clear similarities to the induction of Epo expression in these cells.

Vascular endothelial growth factor (VEGF) is a potent and specific mitogen for vascular endothelial cells and also increases vascular permeability. In glioblastoma multiforme tumors, expression of the gene appeared to be adjacent to necrotic regions, suggesting that expression might be related to local oxygenation (Shweiki et al. 1992). In cultured glioma (C6) cells, fibroblasts (mouse L cells), rat heart primary cultures and skeletal muscle myoblasts VEGF expression was induced by hypoxia (Shweiki et al. 1992). Importantly, cycloheximide very substantially reduced the response to hypoxia. Further investigations in Hep3B cells demonstrated induction at 1\% oxygen of both VEGF and of Epo. VEGF message was induced by cobaltous ions and the response was inhibited by $10 \%$ carbon 
monoxide, thus showing important similarities to the regulation of the Epo gene (Goldberg and Schneider 1994). More recently, two groups have shown induction of VEGF expression by hypoxia and cobaltous chloride in a range of cell types (Minchenko et al. 1994a; Gleadle et al. 1995). In rat tissues hypoxia or anaemia increases VEGF expression (Minchenko et al. 1994a).

In addition to interest in these angiogenic growth factors, two vasoactive substances, adenosine and endothelins, show some evidence of hypoxic regulation. The endothelins are powerful vasoconstrictors, and it is recognised that vasoconstriction (at least in the pulmonary bed) is an important response to hypoxemia. In cultured HUVEC cells, $1 \%$ oxygen induces preproendothelin expression (Kourembanas et al. 1991). This response to hypoxia was demonstrated to be transcriptional. Adenosine has powerful vasomotor actions. Ecto-5'-nucleotidase ( $\left.5^{\prime} \mathrm{NT}\right)$ is a surface enzyme which produces adenosine from AMP. Expression of this enzyme is increased in anaemia in the renal interstitium and overlaps with (but is not identical to) the distribution of Epo expression (Le Hir et al. 1989; Bachmann et al. 1993; Chapter 5).

\section{Transcription factors}

Two groups have demonstrated that hypoxia produces a rapid, high level induction of mRNA for the proto-oncogenes c-Fos, c-Jun, Jun-D and Jun-B. In contracting cardiac myocytes cultured at oxygen tensions below $0.2 \%$ this occurred within one hour, and was demonstrated to occur before ATP depletion (Webster et al. 1993). In terms of similarities with Epo regulation, in Hep3B cells induction was demonstrated within one hour at $1 \%$ oxygen, and induction was also demonstrated in response to $100 \mu \mathrm{M}$ cobaltous ions (Goldberg and Schneider 1994). The early nature of this response to hypoxia, and the known transcriptional activity of these proteins, raised the possibility that this response could be involved in modulating other genes, for instance Epo and VEGF. The latter does have AP-1 binding sites in the 5 ' flanking region. However, phorbol esters markedly increase expression of the fos and jun proto-oncogene families and upregulate VEGF expression, but are known to downregulate Epo expression (Jelkmann et al. 1991, Kurtz et al. 1992). Furthermore, the minimal elements required to convey a hypoxic response from both Epo (Appendix 1) and VEGF (Minchenko et al. 1994b) do not include AP-1 binding sites. 


\section{Cellular metabolism}

Several genes for glycolytic enzymes are transcribed at an increased level in response to hypoxia (Webster 1987). A recent study has shown that the mRNA of the widely-expressed glycolytic enzyme phosphoglycerate kinase-1 (PGK-1) is induced by hypoxia (Firth et al. 1994). Sequence from the 5 ' flanking region confers hypoxia and cobalt inducibility of expression on heterologous promoters and reporter genes. This response was not influenced by cyanide, but was abrogated by cycloheximide. Furthermore, the functionally minimal critical region of 18 base pairs defined in transient transfection studies showed substantial sequence homology to the critical region of the Epo enhancer. In electrophoretic mobility shift assays a 24 base pair oligonucleotide derived from this region of PGK-1 formed a similar (or identical) hypoxically-induced complex to a 24 base pair probe from the Epo enhancer, and the oligonucleotides showed cross-competition. This provides strong evidence that PGK-1 is controlled by a closely similar, or identical, hypoxic control mechanism to that controlling Epo production in hepatoma cells. In addition, hypoxic regulation of LDHA has been demonstrated (Firth et al. 1994). This is the gene for the M subunit of lactate dehydrogenase and catalyses the interconversion of pyruvate and lactate. More recently, several other genes for glycolytic enzymes (phosphofructokinase, aldolase A, enolase and pyruvate kinase $M$ ) have been shown to be regulated by hypoxia and/or to have HIF-1 binding sites (Semenza et al. 1994). By contrast, glucagon-induced expression of the gluconeogenic enzyme phosphoenol pyruvate carboxykinase (PEPCK) in isolated hepatocytes is reduced in response to hypoxia ( $8 \%$ compared to $16 \% \mathrm{O}_{2}$ ). Interestingly, pretreatment with cobalt $(50 \mu \mathrm{M})$ was also shown to reduce expression of PEPCK in this system (Kietzmann et al. 1992). This enzyme is a major rate-governing step in gluconeogenesis.

In the liver, there is higher expression of gluconeogenic enzymes in the well-oxygenated periportal region, whereas glycolytic enzymes are expressed at a higher level in the relatively hypoxic pericentral region (Jungermann and Katz 1989). Culturing hepatocytes in hypoxia (4\% oxygen) increases glycolysis and reduces gluconeogenesis (Wölfle and Jungerman 1985). Given the experimental findings above, it is attractive to speculate that the metabolic zonation in the liver is at least partially due to the hypoxic control mechanism demonstrated in this chapter. 


\section{Miscellaneous genes}

Physiological responses to hypoxia include hyperventilation. This response is mediated via the carotid body. Hypoxia causes the Type 1 carotid body cells to depolarise and release dopamine. Tyrosine hydroxylase is the rate limiting step in dopamine synthesis. Recently it has been shown by in situ hybridisation that there is a five-fold increase in the level of mRNA for this gene in the rat carotid body in response to normobaric hypoxia (CzyzykKrzeska et al. 1992). No response was seen to reducing blood oxygen content (rather than $\mathrm{pO}_{2}$ ) by exposure to carbon monoxide or by hypercapnia (which also stimulates ventilation), indicating that oxygen was the sensed variable. Using a rat phaeochromocytoma cell line (PC12), hypoxic induction of tyrosine hydroxylase expression was demonstrated in 5\% oxygen in tissue culture, and this response was demonstrated to be transcriptional by nuclear run-off using hypoxic nuclei. Transient transfection experiments demonstrated $300 \mathrm{bp}$ of sequence $5^{\prime}$ to the gene to be capable of conferring hypoxic regulation on a promoterless CAT construct in PC12 cells, but not in Cos cells (Czyzyk-Krzeska et al. 1994). Additionally, pulse-chase experiments demonstrated that the mRNA for tyrosine hydroxylase was stabilised by hypoxia.

Expression of the heme oxygenase gene in rat kidney is greatly increased (about forty fold) following the administration of cobalt chloride. The response to anaemia and to hypoxia was not described (Abraham et al. 1991).

\subsection{CONCLUSIONS}

In conclusion the experiments described in this chapter demonstrate the widespread presence of a hypoxic-sensing system which is closely similar (or identical) to that which operates to control Epo production in hepatoma cells.

This led to the proposal that this system (or closely related group of systems) was involved in the regulation of a wide range of genes influencing local blood supply and cellular metabolism. Subsequent work has demonstrated that the PGK-1 gene, genes for other glycolytic enzymes and the VEGF gene are regulated by a hypoxic mechanism which is similar or identical to that regulating Epo. In addition there are a considerable number of other candidate genes where suggestive similarities have been described.

It remains possible that a closely related family of transcription factors is involved in these responses, and that they are similar rather than identical. Resolution of this will require generation of signal pathway mutants or isolation of genes encoding the factors involved. 
It is unknown to what extent this response mechanism is conserved in non-mammalian species. There are well-characterised transcriptional control mechanisms which respond to hypoxia in bacteria and in yeast. How these relate to this mammalian system is not known. There are many striking examples of homology between transcription factors in different species and it seems very likely that this response system will not be confined to mammals. 


\section{Determination of mouse Epo sequence sufficient to direct appropriate expression of a marker gene}

\subsection{INTRODUCTION}

A major problem in the study of Epo gene expression is the failure to identify the Epoproducing cells in the kidney, and the lack of a renal cell culture line showing regulated Epo expression. The experiments described in Chapter 3 indicate that some aspects of Epo gene expression are amenable to study in a wide range of mammalian cell culture systems. In addition they demonstrate that an "oxygen-sensing" mechanism capable of regulating gene expression is not a specific property of specialised Epo-producing cells. Nevertheless, other aspects of Epo gene expression, in particular the basis of the striking tissue-specificity, clearly cannot be studied using the 3' enhancer element alone. As discussed in Section 1.4, transgenic experiments allow the study of a region of DNA in the context of chromatin structure, methylation and other cis-acting elements. Furthermore, the expression of transgenes can be studied in appropriate physiological contexts. Thus in the case of the Epo gene, transgenic experiments allow investigation of the response to anaemia and to hypoxaemia. In addition, tissue distribution and developmental aspects could be studied. It would also be possible to study the cellular distribution of expression within organs (in particular the liver and kidney).

As discussed in Chapter 1, direct immunohistochemical localization of Epo synthesis has not proved possible, most probably because of lack of significant storage at this site. In situ hybridization studies have produced discrepant results. It is, however, clear that the cells which synthesise Epo mRNA lie within the renal cortex and the majority of studies have indicated that they lie within the renal interstitium (Lacombe et al. 1988; Koury et al. 1988b; Koury et al. 1989; Schuster et al. 1992). Further characterization has been difficult. Consequently, in this thesis a different approach has been adopted in order to localise these cells. In transgenic mice integration of a marker gene together with appropriate cis-acting control sequence permits tissue-specific expression of the marker gene. This has then been used to localise expression by visualization of the marker gene product, or to promote tumour formation and cellular transformation using an oncogenic protein (Hanahan 1985; Palmiter and Brinster 1986). 
This chapter is therefore concerned with defining cis-acting sequence capable of directing appropriate expression of a marker gene in transgenic mice. In the current experiments, two criteria have been used to define appropriate expression of the marker gene. Firstly, induction of expression by hypoxia and anaemia. Secondly, the tissue distribution of expression. In each case expression has been measured by RNase protection analysis of total RNA extracted from homogenised organs.

The transgenic constructs used were designed so that a single RNase protection assay would detect and distinguish transgene mRNA and native Epo mRNA. This consequently allowed a direct and quantitative comparison of the two species. The initial choice of cis-acting sequence was based on using as extensive flanking sequence as possible and using murine DNA. The transgenes therefore contained $16.5 \mathrm{~kb}$ of mouse DNA isolated from a bacteriophage library, which included the entire mouse Epo gene, $9 \mathrm{~kb}$ of 5 ' flanking sequence and $3.5 \mathrm{~kb}$ of $3^{\prime}$ flanking sequence. Two different constructs were employed; Epo-M and Epo-TAg.

The ability of this sequence to direct expression was not known. There are several areas of conservation between mouse and man in the published $8 \mathrm{~kb}$ of sequence (Shoemaker and Mitsock 1986; McDonald et al. 1986; Galson et al. 1993). Each of these conserved regions is likely to subserve important regulatory functions. The first transgenic construct therefore included the smallest modification compatible with detection of the transgene in genomic DNA and detection of mRNA. This construct, Epo-M, was marked by insertion of BglII linkers ( 8 base pairs) in the 5 ' untranslated region. Expression studies performed on transgenic mice bearing this construct indicated that it was inducibly expressed in liver and kidney. However, the level of expression in the liver was substantially greater than that in the kidney.

In the second construct, Epo-TAg, viral sequence from simian virus 40 (SV40) coding for T $\mathrm{Ag}$ was inserted at the engineered BglII site in Epo-M. Mice bearing this transgene expressed the gene inducibly in liver and kidney; interestingly they did not over-express the transgene in the liver compared to the kidney. An important observation was that in one line (Epo$\mathrm{TAg}^{\mathrm{H}}$ ) the Epo-TAg DNA had integrated in a targeted fashion. The result of this insertion was that the marker gene was now flanked by all potential cis-acting sequence.

For clarity, this chapter first describes the distribution of expression of the native Epo gene; this data is derived from observations both on transgenic and normal animals. Following this, the Epo-M and Epo-TAg transgenic mice and the pattern of expression of the transgenes are described. The expression studies demonstrate that in Epo-TAg mice, and particularly 
Epo-TAg ${ }^{\mathrm{H}}$ mice, the fusion gene is a suitable marker of native Epo gene expression. Further experiments discussing the localisation of Epo-producing cells in the kidney and liver form the subject of Chapters 5 and 6.

In addition to establishing that Epo-TAg directs appropriate expression of a marker gene, the data from these experiments allow important conclusions to be drawn concerning the localisation of cis-acting sequence involved in tissue-specific expression of the Epo gene. These findings are discussed in this chapter. In addition, transient transfection experiments using parts of the Epo 5' flanking sequence are described.

\subsection{METHODS}

The constructs used are described in Chapter 2. The sequence of the promoter and Epo 5' UTR in Epo-M and Epo-TAg is given in Appendix 2. Figure 4.1 shows Epo-M and EpoTAg schematically, together with the specific riboprobes employed.

A.

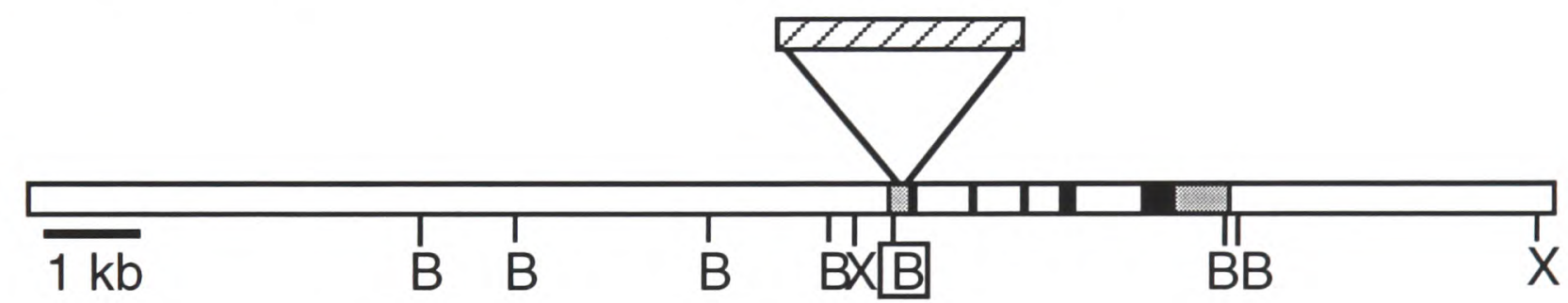

B.
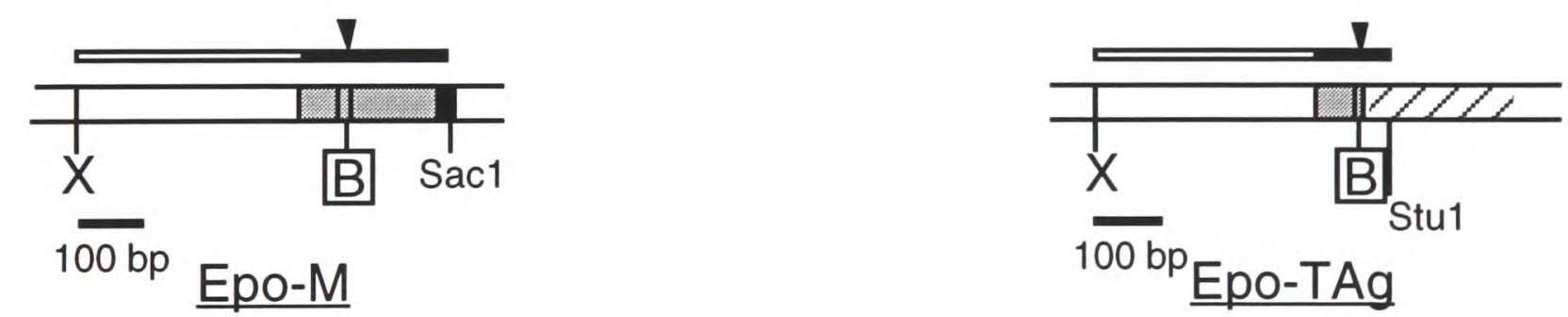

Figure 4.1 - Transgenic constructs and riboprobes. (A) The $16.5 \mathrm{~kb}$ of cloned DNA is shown, with the Epo gene and its 5 exons (untranslated sequence is shown shaded, translated sequence is solid). BglII $(B)$ and XbaI $(X)$ restriction sites are indicated. A novel BglII site (boxed) was inserted in Epo-M to mark the transgene and its transcripts. In Epo-TAg a $2.7 \mathrm{~kb}$ BglLBamHI restriction fragment of SV4O DNA was inserted as shown (hatched).

(B) Riboprobes. For detection of Epo-M transcripts, an Xbal/Sacl fragment of Epo-M was used to generate a riboprobe which crossed the transcription start site. The protected fragment from correctly initiated transcription $(221 \mathrm{bp}$ ) is shown as a solid bar. Endogenous Epo transcripts lacking the Bgl II insert were cleaved into two fragments (arrowhead) of 148 and $65 \mathrm{bp}$. For detection of Epo-TAg transcripts, an Xbal/Stul fragment of Epo-TAg was used to generate a riboprobe which crossed the transcription start site and included SV40 sequence. The protected fragment from correctly initiated transcription (120 bp) is shown as a solid bar. Endogenous Epo mRNA protected 65 bp (arrowhead).

The studies to determine the expression of native Epo mRNA were performed using the mouse Epo probe on non-transgenic MF1 mice or non-transgenic littermates (8 animals). This probe protects a $213 \mathrm{bp}$ fragment. In addition, considerable information on native Epo 
gene expression was obtained from the Epo-M transgenic animals (22 animals included); in these animals native Epo mRNA protected a 148 bp fragment; so sensitivity was only marginally reduced compared to use of the mouse Epo probe.

Transgene expression in each line was determined in at least four mice; one transgenic mouse of each sex under both baseline and acutely anaemic conditions. The RNase protection analyses were performed with the riboprobe specific to the relevant transgene. Prior to these experiments a large quantity of kidney RNA from acutely anaemic non-transgenic mice was extracted, pooled and stored in aliquots as a standard preparation. One standard aliquot was analysed in each RNase protection assay, to allow comparison between assays. In individual samples, abundance of transgene mRNA relative to total RNA, and also relative to native Epo mRNA could be determined. In quantitative terms, the comparison of transgene to native Epo mRNA in a single sample was very reliable, since almost all possible variables in the purification and assay system would affect both RNA species equally. By contrast, comparison of the level of expression between organs is somewhat less reliable.

\subsection{RESULTS}

\subsubsection{Tissue distribution of Epo gene expression in the mouse}

A total of 30 mice were studied for baseline Epo expression or following induction of expression using normobaric hypoxia or haemolytic anaemia. An illustative RNase protection assay is shown in Figure 4.2. The pattern of expression in normal and stimulated mice is shown in Table 4.1. As anticipated from previous studies, in normal mice expression was demonstrable in the kidney using $100 \mu \mathrm{g}$ of total RNA. There was high level induction by anaemia in the kidney, and in anaemic animals a quantifiable level of mRNA was present in the liver. In addition to these sites, mRNA was also observed in the brain, testis and preputial gland of normal mice at a level which was similar to that observed in the kidney. Although quantitative studies were not performed, anaemia consistently appeared to result in increased levels of mRNA in each of these organs. The level of induction appeared to be substantially lower than is observed in the kidney or liver, but was consistently in the region of two to three-fold. 


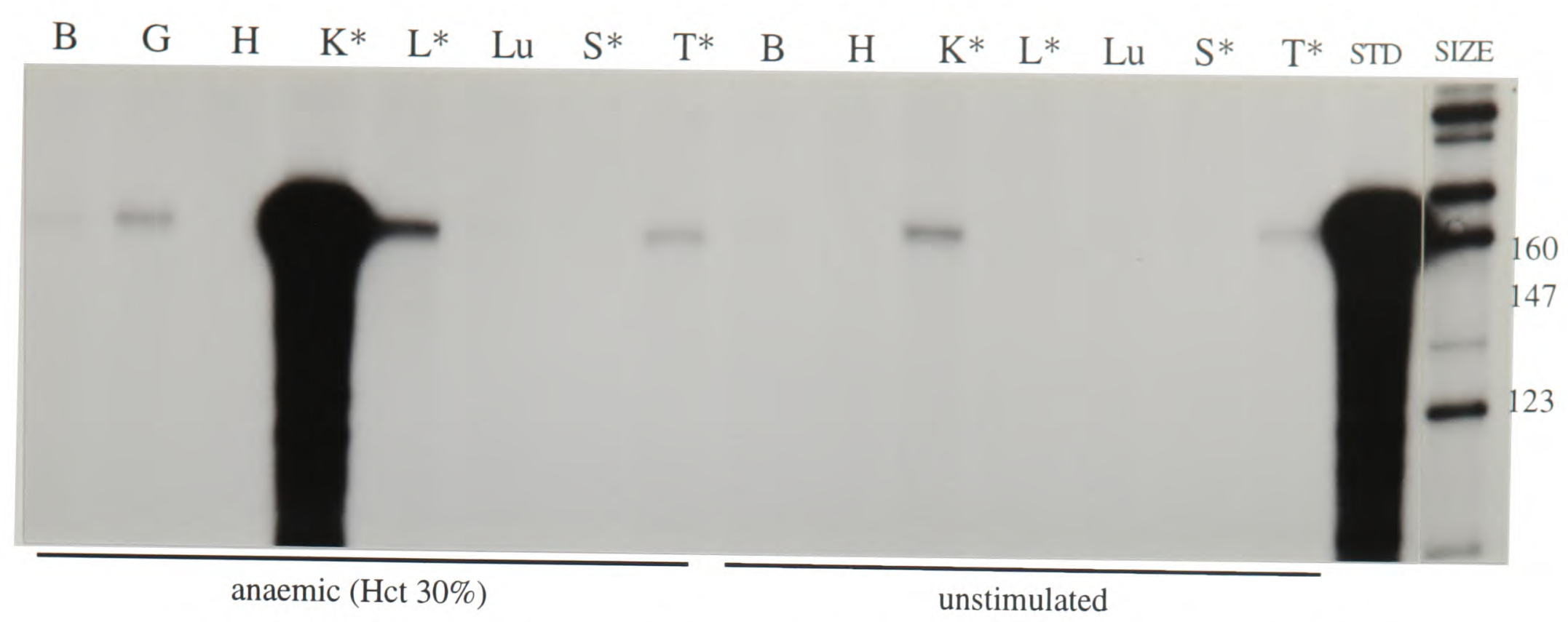

Figure 4.2 - RNase protection assay illustrating the expression of the native Epo gene. RNA from the organs of two male mice of the 35.6 Epo-M line has been hybridised with the Epo-M riboprobe. $K=$ Kidney, $L=$ Liver, $B=$ Brain, $H=$ Heart, $L u=$ Lung, $S=$ Spleen, $G=$ preputial gland, $T=$ Testis. $150 \mu \mathrm{g}$ was analysed except where indicated $*$ when $500 \mu \mathrm{g}$ was used. No expression of the transgene was evident (221 bp fragment). Native Epo mRNA protects a 148 bp fragment of the probe. Expression of the native Epo gene is seen in several organs of the mildly stimulated animal; kidney, liver, preputial gland and testis. In the unstimulated animal the level of expression in the kidney is much lower and none is detected in the liver. Expression is also seen in the testis of this animal, and is just detectable in the brain. In this study high-level inducibility is clear in the kidney and liver. The signal in the brain, and possibly testis, is also seen to be induced by anaemia.

\begin{tabular}{|l|c|c|c|c|c|c|c|c|c|c|}
\cline { 2 - 11 } \multicolumn{1}{c|}{} & $\mathrm{K}$ & $\mathrm{L}$ & $\mathrm{B}$ & $\mathrm{H}$ & $\mathrm{Lu}$ & $\mathrm{S}$ & $\mathrm{G}$ & $\mathrm{T}$ & $\mathrm{O}$ & $\mathrm{U}$ \\
\hline Stimulated & +++ & ++ & + & - & $+/-$ & - & + & + & $+/-$ & - \\
\hline Baseline & + & - & $+/-$ & - & - & - & $+/-$ & $+/-$ & $+/-$ & - \\
\hline
\end{tabular}

Table 4.1 - Summary of expression of the native Epo gene in the mouse. Abbreviations as in Figure 4.2; $O=$ Ovary, $U=$ Uterus. The sensitivity varied somewhat from one assay to another. A total of 20 stimulated (Hct 14-35\%) and 10 unstimulated mice are included in the analysis; not all organs in each case were analysed. The indication of level of expression is somewhat arbitrary; + indicates a level roughly equivalent to that in unstimulated kidney, +++ indicates the level in acutely anaemic kidney. +/- indicates that expression was not reliably detectable and was less than that in unstimulated kidney. In the kidney, liver and brain expression was clearly increased by anaemia. In the testis and preputial gland expression was probably increased somewhat by anaemia, but was less clearly inducible. A very low level of expression in the lung was seen in some of the most anaemic animals. Expression in the ovary did not seem to be related anaemia.

In contrast to these organs, where expression was consistent and was increased by anaemia, some expression was observed in the ovary, but was inconsistent and not clearly related to the level of haematocrit. This may have been partly due to difficulties in handling the small amount of RNA from two mouse ovaries; additionally it is certainly possible that the variation is related to the oestrus cycle. No Epo mRNA was detected in the heart, the uterus or the spleen. In the lung a very low level of transcripts was present in three severely anaemic animals. 
The riboprobes used in these studies extended from the Xbal site (which lies 400 base pairs 5 ' to the transcription start site) to the Sacl site (Appendix 2). In these experiments no evidence of protected lengths other than that predicted were observed; i.e. no evidence was found for other transcriptional start sites or for splicing into this region.

\subsubsection{The site of Epo expression in the brain}

The observation of Epo expression in the brain, which was hypoxically regulated, prompted the question as to whether this was confined to a particular structure or region within the brain. This was not addressed in the mouse, but in the rat, so that dissection was facilitated and an assayable quantity of RNA was obtained. Only anaemic animals were studied. The rats were sacrificed, the brain dissected and separate portions processed in parallel for RNA extraction. The result of this experiment is shown in Figure 4.3. The striking finding is that Epo mRNA appears to be evenly distributed throughout the brain.
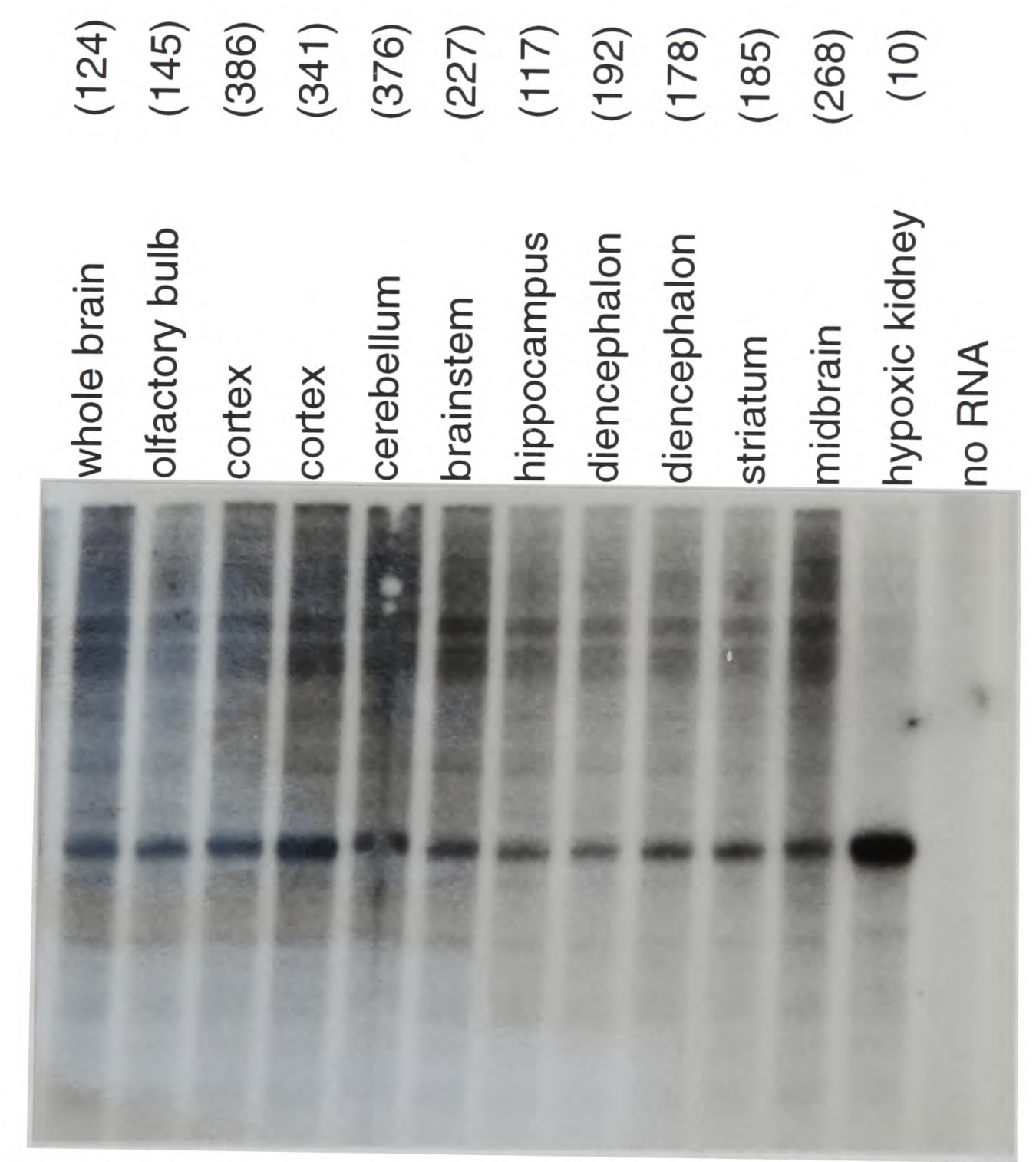

Figure 4.3 - RNase protection assay analysing the distribution of Epo mRNA in the brain of anaemic rats. Rats were rendered acutely anaemic with phenylhydrazine. The brain was dissected and RNA was extracted. The amount of RNA assayed $(\mu \mathrm{g})$ is indicated. A sample of kidney RNA from an acutely hypoxic rat was also analysed. A low level of RNA is seen in all regions of the brain. 


\subsubsection{Epo-M transgenic experiments}

\subsubsection{Identification and characterisation of transgenic lines}

Four transgenic animals were identified among $74 \mathrm{~F}_{\mathrm{O}}$ mice born following intrazygotic injection with Epo-M. One of these four founders (37.6) gave rise to two different types of transgenic $\mathrm{F}_{1}$ offspring, 37.6LC (low copy) and 37.6 HC (high copy). In this founder two separate integrations had presumably occurred. Thus a total of 5 different Epo-M lines were obtained. In each of these 5 lines the transgene was inherited in a Mendelian fashion.

The number of copies of Epo-M was estimated by comparison with a standard curve created by adding serial two-fold dilutions of Epo-M DNA to mouse DNA (Figure 4.4) and is indicated in Table 4.2. In two lines $(37.6 \mathrm{HC}, 198.3)$ the transgene was inserted as a multiple copy array. In the remaining three lines $(35.6,38.5,37.6 \mathrm{LC})$ a single copy of the transgene had integrated.

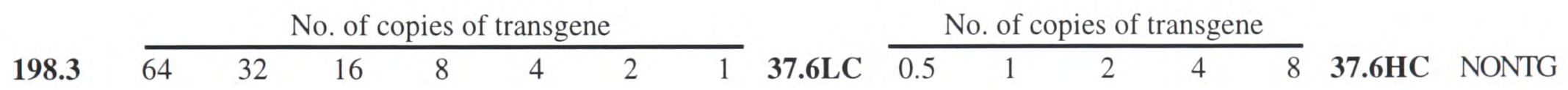

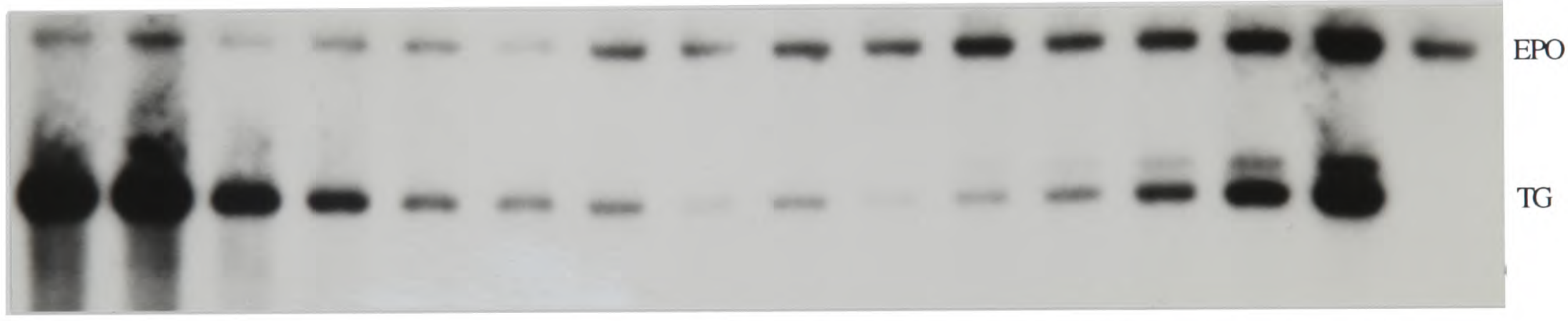

Figure 4.4 - Estimation of Epo-M copy number. Serial dilutions of Epo-M plasmid were made in non-transgenic DNA. From this series, the sample with bands of equal intensity at $4.4 \mathrm{~kb}$ (Epo) and 3.6kb (Epo-M) is taken as equivalent to two copies of the native gene and two copies of the transgene. By comparison with this series, 37.6LC has one copy of Epo-M, 198.3 has 64 copies and 37.6HC has 16 copies.

The 35.6 and $37.6 \mathrm{HC}$ lines each had an additional band to the predicted $3.6 \mathrm{~kb}$ band when a BglII digest was probed with Probe 6. Both these lines therefore included one copy of the transgene which was truncated into the Epo-M gene (see Figure 2.2, p.49).

Further defining the structure of integrated Epo-M transgenes precisely would be difficult, because the only difference from the native Epo gene is the $8 \mathrm{bp}$ insertion forming a BgIII site in the 5'UTR. In the case of the lines bearing multi-copy arrays intense bands of the anticipated length on Xbal and EcoRl digests with Probe 6 demonstrated that many copies of the transgene were intact over at least the $3^{\prime} 13.5 \mathrm{~kb}$ of the $16.5 \mathrm{~kb}$ transgene. Further analysis of these two lines and of 38.5 (which did not express the transgene) were not undertaken. 
The two lines 37.6LC and 35.6 were analysed further. This was undertaken in some detail because $37.6 \mathrm{LC}$ was not expressed in the kidney, in contrast to the other Epo-M lines (see below).

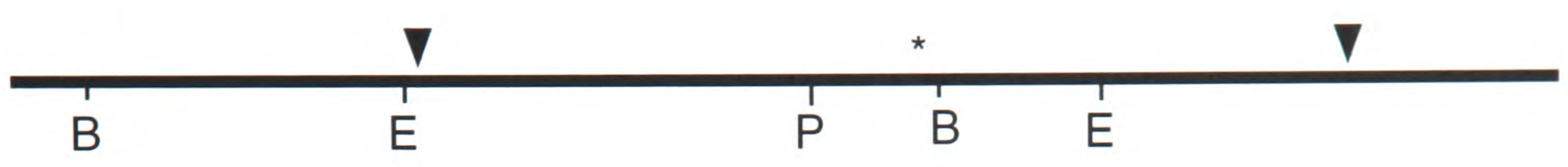

37.6LC

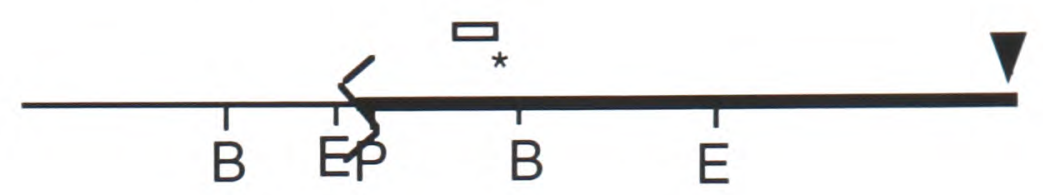

EcoR 1

BamH1 BamH1/Pst1

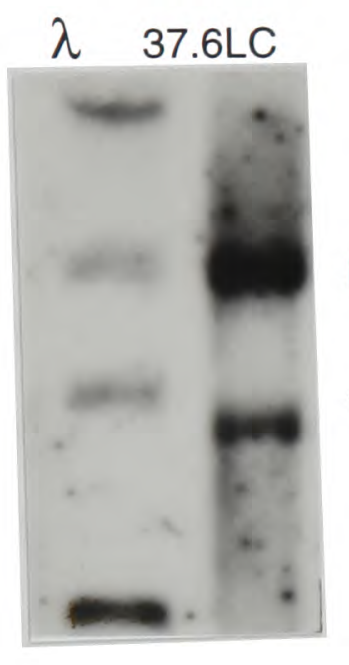

EPO (9KB)

TG (5.4KB)

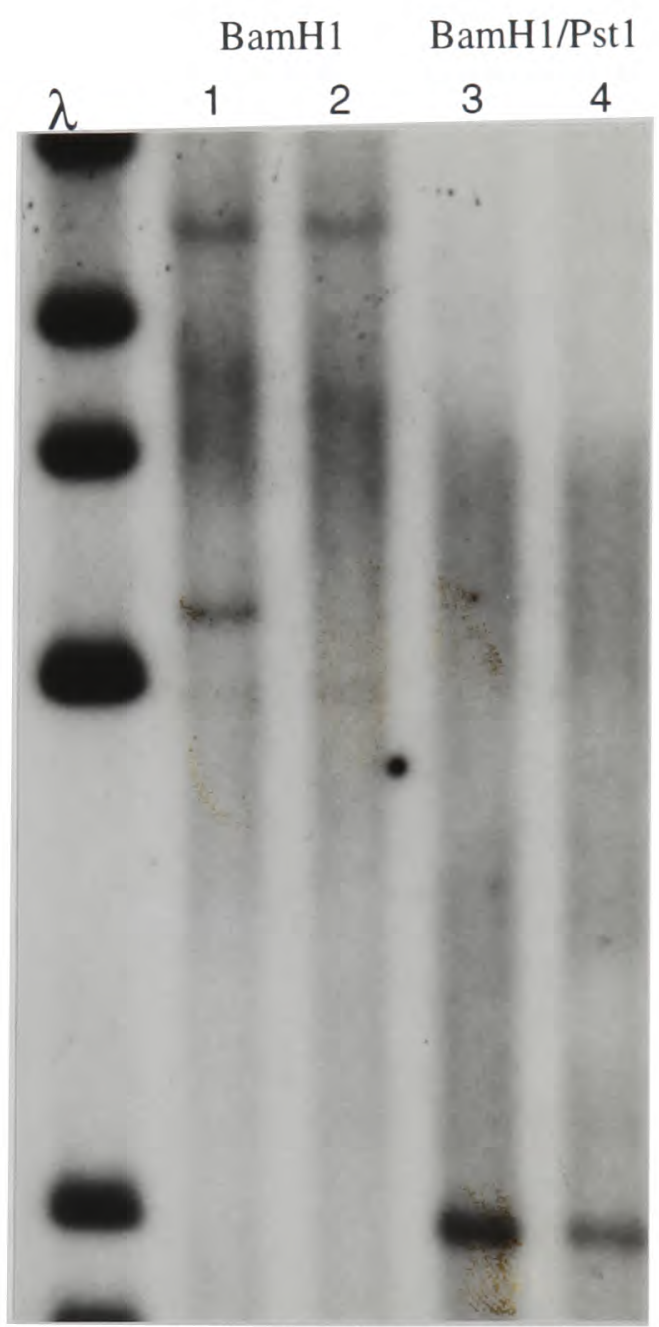

EPO (13.7KB)

TG (4.5KB)

Figure 4.5 - Mapping the truncation of Epo-M in the 37.6LC line. At the top of the figure the Epo locus is shown $(1 \mathrm{~kb}=0.5 \mathrm{~cm})$. Arrowheads indicate the extent of cloned DNA in Epo-M, and $*$ indicates the site of the Bgl2 linkers inserted in Epo-M. Restriction sites; $E=E c o R I$, $B=B a m H 1, P=P$ st1. Restriction sites not relevant to this analysis are not shown. Open box shows the probe used (probe 5; see Figure 2.1, p.46). In the EcoRI digest, a site flanking the transgene is demonstrated by the presence of a $5.4 \mathrm{~kb}$ fragment. In the BamHI digest, a $4.5 \mathrm{~kb}$ fragment is present in 37.6LC DNA (lane 1) but not in non-transgenic DNA (lane 2). This demonstrates the presence of a flanking BamH1 fragment as shown. When DNA is digested with BamH1 and Pstl, this fragment is shortened to $2.2 \mathrm{~kb}$ both in 37.6LC (lane 3) and nontransgenic samples (lane 4). This demonstrates that the Pstl site is preserved in the truncated Epo-M transgene. The 37.6LC transgene is indicated, with the restriction sites; the transgene is indicated by a heavy line, with the breakpoint as shown.

The 37.6LC line was shown to have a 5' truncation of the Epo-M transgene, which could be precisely delineated to between $1.8 \mathrm{~kb}$ and $2.2 \mathrm{~kb} 5$ ' to the transcription start site, as follows. This data is shown in Figure 4.5. (1) Probes 5 and 6 (see Figure 2.1, p. 46) hybridised to an EcoR 1 fragment of $5.4 \mathrm{~kb}$. This places a maximum of $2.2 \mathrm{~kb}$ on the distance from the end 
of the transgene to the transcription start site. (2) Probe 5 hybridised to a BamHl fragment of $4.5 \mathrm{~kb}$; this was shortened to $2.2 \mathrm{~kb}$ by digestion with Pstl and $\mathrm{BamHI}$, indicating that the Pstl site $1.8 \mathrm{~kb} 5$ to the transcription start site was present in the transgene. This places a minimum of $1.8 \mathrm{~kb}$ on the distance from the $5^{\prime}$ end of the transgene to the transcription start site.

The 3' limit of this transgene could not be accurately determined; it certainly extended to the $B g l I I$ site at the 3' end of the gene. The presence of a more intense band on digests with $X$ Xal probed with probe 7 suggested that it was intact to the $3^{\prime}$ end of Epo-M.

By contrast, it was established that the 35.6 line contained one complete copy of the Epo-M transgene. A transgene-specific fragment was demonstrated at the 5 ' end, both with BamHI and BglII. These fragments hybridised to probe 1 and to probe 2, demonstrating that the extreme 5 ' sequence was present in the transgene. At the 3 ' end, certainly intact sequence covering the BglII fragment was present. In addition, the intensity of the band observed in $\mathrm{Xbal}$ digests suggested that the $7.5 \mathrm{~kb}$ Xbal fragment was intact in one of the two copies. Thus, although one copy of the transgene was truncated, all the sequence injected was present.

\subsubsection{Phenotype and haematocrits}

Haematocrits from transgenic and non-transgenic animals of the same stock are shown in Table 4.2. Among the Epo-M lines only 198.3 was polycythemic. The $37.6 \mathrm{HC}$ mice may also have had a slightly higher haematocrit than controls. In the case of the 38.5 and 35.6 too few measurements were performed for a comparison to be valid.

\begin{tabular}{|l|c|c|c|}
\cline { 3 - 4 } \multicolumn{1}{c|}{} & \multicolumn{2}{c|}{ Haematocrit } \\
\hline Line & Copy no. & $<2$ months & $>2$ months \\
\hline Non Tg & n/a & $41.6 \%+/-4.8(176)$ & $44.2 \%+/-5.2(75)$ \\
\hline 35.6 & 1 & $55.5 \%+/-2.5(2)$ & $44.6 \%+/-3.8(17)$ \\
\hline 38.5 & 1 & $46.0 \%+/-9.0(2)$ & $51.0 \%+/-7.1(2)$ \\
\hline $37.6 \mathrm{LC}$ & partial & $42.3 \%+/-8.7(4)$ & $44.7 \%+/-5.9(20)$ \\
\hline $37.6 \mathrm{HC}$ & 16 & $43.7 \%+/-5.4(7)$ & $47.4 \%+/-6.9(16)$ \\
\hline 198.3 & 64 & $59.8 \%+/-8.8(25)^{*}$ & $76.2 \%+/-6.1(37)^{*}$ \\
\hline
\end{tabular}

Table 4.2 - Estimated transgene copy number and haematocrits of Epo-M transgenic mice. Figures given for haematocrit are mean $+/-s . d$., with the number of observations in parentheses. * indicates significant difference from non $\mathrm{tg}(p<0.05$, $t$-test with correction for multiple comparisons).

In a single polycythaemic 198.3 mouse Epo was measured by RIA; this mouse had an Epo level of $33 \mathrm{mIU} / \mathrm{ml}$ with a haematocrit of $80 \%$. Thus, in the context of severe polycythaemia 198.3 mice continue to produce measurable levels of Epo. Interestingly, polycythaemia was much less evident in young 198.3 mice (Figure 4.6). 


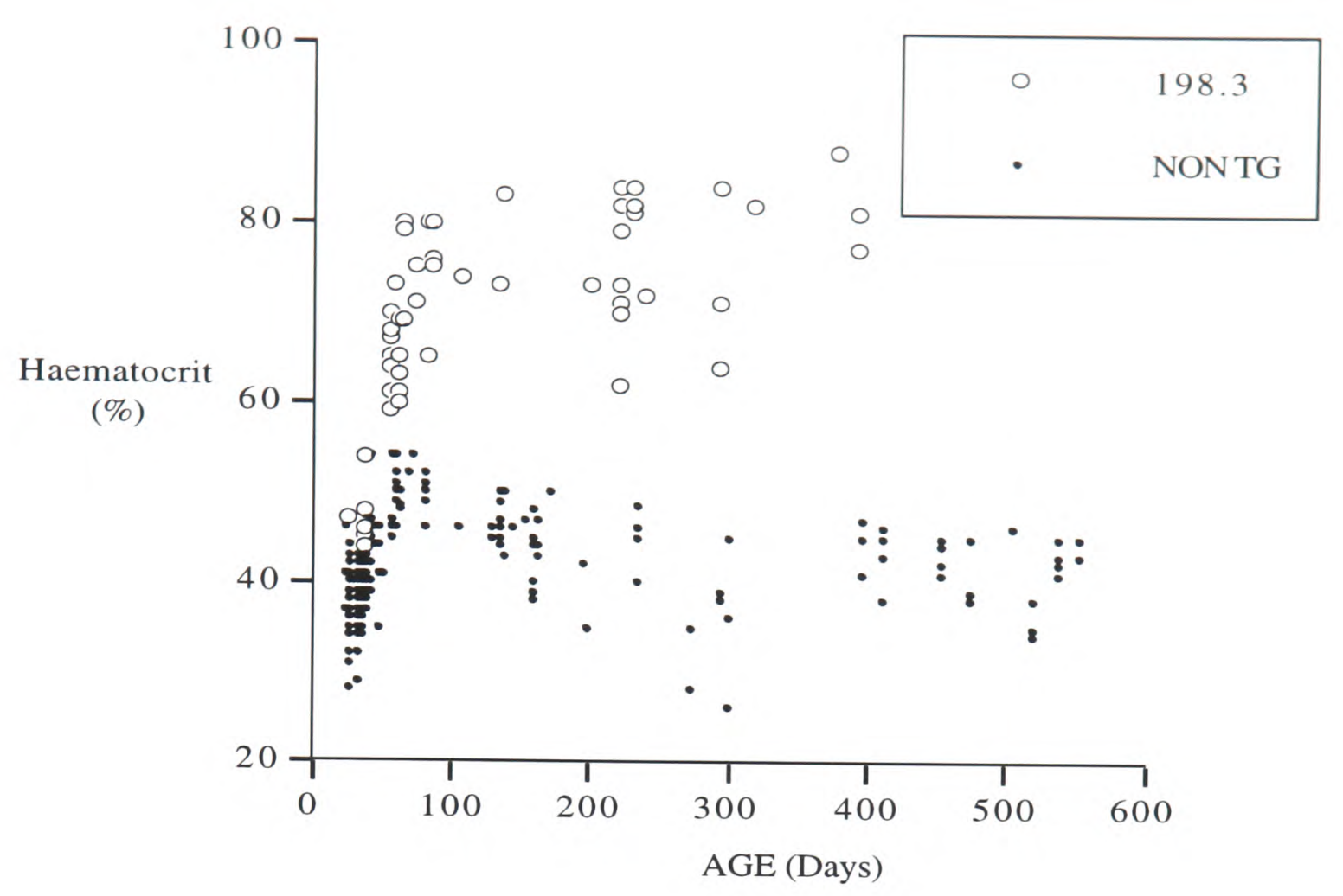

Figure 4.6 - Haematocrits of 198.3 transgenic mice and non-transgenic mice plotted against time. 198.3 mice become markedly polycythaemic at around the time of sexual maturity.

\subsubsection{Tissue distribution of expression of the Epo-M transgene}

Transgene expression was studied at the mRNA level using RNAse protection assays. Male and female animals were studied both in the unstimulated state and after induction of acute haemolytic anemia with phenylhydrazine. Ten organs were studied; kidney, liver, brain, heart, lung, spleen in all animals; preputial gland and testis in males, and ovary and uterus in females. Expression in the acutely anaemic animals is summarised in Table 4.3.

\begin{tabular}{|l|c|c|c|c|c|c|c|c|c|c|}
\hline \multicolumn{1}{|c|}{ Line } & $\mathrm{K}$ & $\mathrm{L}$ & $\mathrm{B}$ & $\mathrm{H}$ & $\mathrm{Lu}$ & $\mathrm{S}$ & $\mathrm{G}$ & $\mathrm{T}$ & $\mathrm{O}$ & $\mathrm{U}$ \\
\hline $37.6 \mathrm{LC}$ & - & + & + & - & - & - & - & - & + & + \\
\hline 38.5 & - & - & - & - & - & - & - & - & $\mathrm{NE}$ & $\mathrm{NE}$ \\
\hline 35.6 & + & + & + & - & - & - & - & - & - & + \\
\hline $37.6 \mathrm{HC}$ & + & $+^{*}$ & $+^{*}$ & - & - & - & + & + & $+^{*}$ & $+^{*}$ \\
\hline 198.3 & + & $+^{*}$ & $+^{*}$ & - & - & - & + & + & $+^{*}$ & $+^{*}$ \\
\hline
\end{tabular}

Table 4.3 - Tissue distribution of Epo-M expression in transgenic mice treated with phenylhydrazine. The notation for organs is as in Table 4.1. + indicates correctly initiated transcripts, - indicates no correctly initiated transcripts. $*=$ additional incorrectly initiated transcripts. $N E=$ not examined.

Of the four lines containing the intact Epo-M transgene, one (38.5) showed no expression. In the remaining three, inducible expression was observed in both liver and kidney. Although this pattern of dominant hepatic and renal expression was similar to that of the endogenous Epo gene, differences were observed. Expression of the transgene in the liver of anaemic mice was many fold greater than that of the endogenous gene. In contrast, transgene 
expression in the kidney was lower than that of the endogenous gene; the relative abundance of transgenic Epo mRNA being less than $30 \%$ of the abundance of endogenous Epo mRNA in lines 35.6, 198.3 and 37.6HC (Table 4.4 and Figure 4.7).

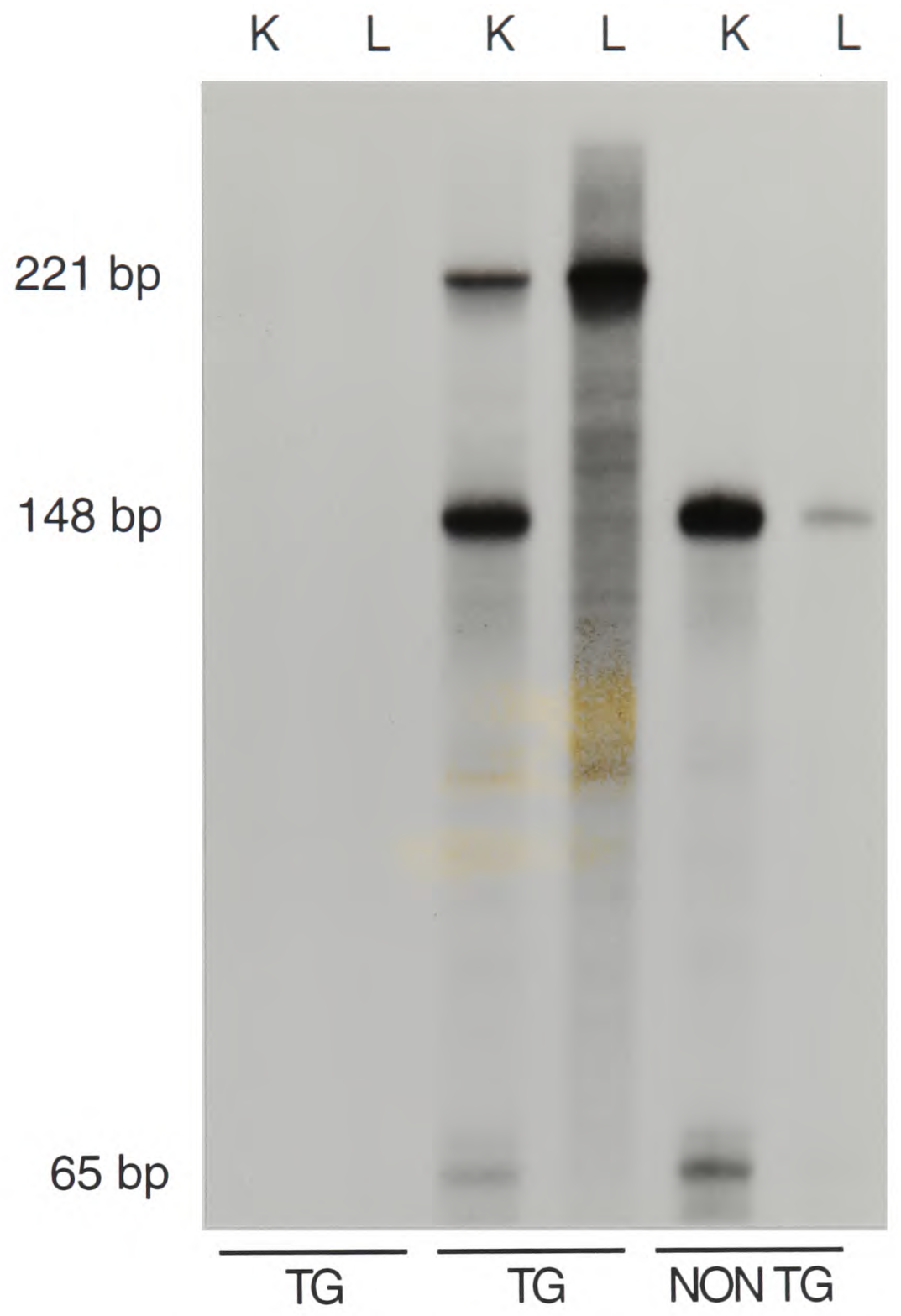

Figure 4.7 - RNAse protection assay using the Epo-M riboprobe showing inducible expression in the kidney $(K)$ and liver $(L)$ of $37.6 H C(T G)$ and non-transgenic (non $T G$ ) mice. The first two lanes show RNA from an unstimulated 37.6HC mouse; no transcripts are seen. The next two lanes show RNA from an acutely anaemic 37.6HC mouse; one band is seen from the transgene $(221 \mathrm{bp})$ and two bands (148bp and 65b ) are seen to arise from the endogenous gene in kidney. In liver, the transgene is over-expressed and there are additional aberrantly initiated transcripts. In the right hand two lanes, RNA from an acutely stimulated nontransgenic mouse is shown for comparison.

\begin{tabular}{|l|c|c|c|}
\hline \multicolumn{1}{|c|}{ Line } & Copy no. & Kidney & Liver \\
\hline $37.6 \mathrm{LC}$ & partial & - & $26 \%$ \\
\hline 35.6 & 1 & $<3 \%$ & $35 \%$ \\
\hline $37.6 \mathrm{HC}$ & 16 & $22 \%$ & $43 \%$ \\
\hline 198.3 & 64 & $27 \%$ & $144 \%$ \\
\hline
\end{tabular}

Table 4.4 - Quantitation of Epo-M expression in the kidney and liver of acutely anaemic animals. The figures are for Epo-M transcripts, relative to the level of native Epo mRNA in the kidney of the same animal. In all cases the level of Epo-M expression was higher in the liver than in the kidney (in contrast to native Epo mRNA which is expressed at a much lower level in liver than in kidney).

Relative to induced expression in kidney and liver, low levels of transgene and endogenous Epo gene expression were detected by RNase protection assays in brain, ovary, testis and preputial gland. In general, the levels of transgene mRNA were somewhat greater than that 
of endogenous Epo mRNA in these organs. A high constitutive level of aberrant expression was present in the uterus of two lines, $37.6 \mathrm{HC}$ and 198.3. In the liver, in addition to the correctly initiated transcripts, some aberrant transcription was observed in $37.6 \mathrm{HC}$ and 198.3 mice; this was hypoxically regulated (Figure 4.7). When a polycythaemic 198.3 animal was analysed, there was no evidence of expression of Epo-M in the kidney or liver. However, there was evidence of expression in the ovary and uterus.

In the $37.6 \mathrm{LC}$ line in which approximately $7.2 \mathrm{~kb}$ of $5^{\prime}$ sequence was deleted from Epo-M, highly inducible expression was observed in liver at a level similar to that seen in the other lines, but there was no expression in kidney. Inducible expression was also observed in the brain of 37.6LC animals.

\subsubsection{Epo-TAg transgenic experiments}

\subsubsection{Identification and characterisation of transgenic lines}

Of $142 \mathrm{~F}_{\mathrm{O}}$ mice born following injection with Epo-TAg, 6 were transgenic. One of these (134.3) gave rise to a single transgenic offspring (191.10) among 54 live born pups from 5 litters. This strongly suggests that in the germline the founder animal was a mosaic; it is therefore likely that the exogenous DNA integrated late. This offspring then gave rise to two types of transgenic offspring, one bearing a multicopy transgene $(134.3 \mathrm{HC})$ and the other a single copy homologous recombination which replaced one copy of the endogenous Epo gene (134.3LC). One other Epo-TAg founder (188.1) gave rise to two different types of offspring (188.1LC and 188.1HC). Thus, in total, eight Epo-TAg lines were obtained. Proof of homologous recombination of the transgene in line 134.3LC is discussed in chapter 8. In this line, subsequently designated Epo-Tag $\mathrm{H}$, the SV40 viral DNA was inserted in the 5 ' UTR of the native Epo gene. The extent of the gene conversion or double crossover which gave rise to this event could not be precisely defined. Analysis of the flanking DNA suggests that the native Epo locus was not modified in any way apart from the $2.7 \mathrm{~kb}$ insertion (see chapter 8 ). In this line the marker gene was therefore flanked by all the same sequence as the native Epo gene. In addition, the insertion results in the placement of SV40 polyadenylation signals 5 ' to Epo coding sequence; thus although the Epo sequence was not modified, it was likely that the native Epo gene would not be expressed normally (see below). 


\subsubsection{Phenotype and haematocrit of Epo-TAg transgenic animals.}

Of the Epo-TAg lines, $176.5,134.3 \mathrm{HC}$ and $188.1 \mathrm{HC}$ were mildly polycythemic, as shown in Table 4.5. This polycythaemia was more variable than that observed in 198.3 Epo-M transgenic animals; some of the Epo-TAg mice of these lines never developing high haematocrits.

\begin{tabular}{|l|l|l|}
\hline \multicolumn{1}{|c|}{ Line } & \multicolumn{1}{c|}{$<2$ months } & \multicolumn{1}{c|}{$>2$ months } \\
\hline Non Tg & $41.6 \%+/-4.8(176)$ & $44.2 \%+/-5.2(75)$ \\
\hline 99.5 & - & $46.5 \%+/-5.4(17)$ \\
\hline $134.3 \mathrm{HC}$ & $43.6 \%+/-1.9(7)$ & $68.4 \%+/-17.0(5)^{*}$ \\
\hline 175.5 & $44.0 \%+/-1.0(2)$ & $46.1 \%+/-6.3(72)$ \\
\hline 176.5 & $47.0 \%+/-4.0(6)$ & $62.8 \%+/-13.9(39)^{*}$ \\
\hline $188.1 \mathrm{HC}$ & $46.9 \%+/-7.1(39)$ & $57.3 \%+/-13.6(24)^{*}$ \\
\hline $188.1 \mathrm{LC}$ & $36.6 \%+/-4.4(13)$ & $41.1 \%+/-7.9(8)$ \\
\hline 188.3 & $39.7 \%+/-0.5(3)$ & $44.1 \%+/-5.7(27)$ \\
\hline Epo-TAg $\mathrm{TA}^{\mathrm{H}}$ heterozygote & $35.3 \%+/-4.1(158)^{*}$ & $37.3 \%+/-5.6(150)^{*}$ \\
\hline Epo-TAg ${ }^{\mathrm{H}}$ homozygote & $17.5 \%+/-4.0(101)^{*}$ & $18.8 \%+/-2.7(109)^{*}$ \\
\hline
\end{tabular}

Table 4.5 - Haematocrits of Epo-TAg transgenic mice. Figures given are mean+/-s.d., with the number of observations in parentheses. * indicates significant difference from non tg ( $p<0.05, t$-test with correction for multiple comparisons).

Heterozygous Epo-TAgH mice were significantly anaemic when compared to non-transgenic controls. Homozygous Epo-TAgH mice, in which both copies of the Epo gene are modified, were severely anaemic; however, they appeared in litters at the predicted frequency, suggesting that fetal and neonatal viability were normal. A further striking phenotypic abnormality was the observation of preputial gland enlargement in male mice of most of the transgenic Epo-TAg mice; 134.3HC, 176.5, 188.1LC, 188.1HC, 188.3 and to a lesser extent Epo-TAgH homozygotes. Epo levels were measured by RIA in two polycythaemic mice (from the 176.5 and $188.1 \mathrm{HC}$ lines; haematocrit $80 \%$ in each case). 120 and 180 $\mathrm{mIU} / \mathrm{ml}$ were detected, indicating the presence of substantial levels of Epo in the serum in spite of the polycythaemia.

\subsubsection{Tissue distribution of transgene expression in Epo-TAg mice}

Six out of seven lines bearing the Epo-TAg transgene at heterologous insertion sites showed a tissue-specific pattern of transgene expression which was very similar to that of the endogenous Epo gene; one line showed no expression (Figure 4.8 and Table 4.6). 


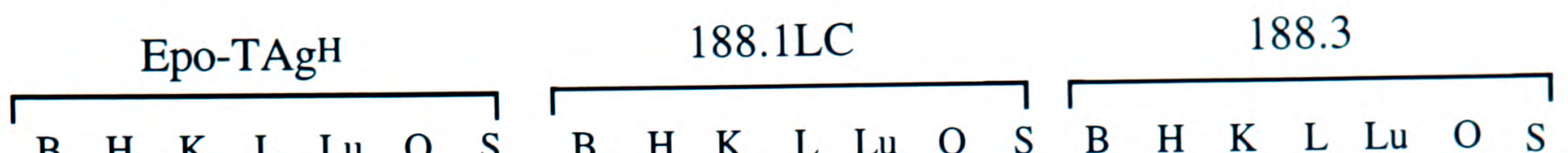

120
B $\quad \mathrm{H} \quad \mathrm{K} \quad \mathrm{L} \quad \mathrm{Lu} \quad \mathrm{O}$
B $\quad \mathrm{H} \quad \mathrm{K} \quad \mathrm{L} \quad \mathrm{Lu} \quad \mathrm{O}$
B $\mathrm{H} \quad \mathrm{K}$
STD

65

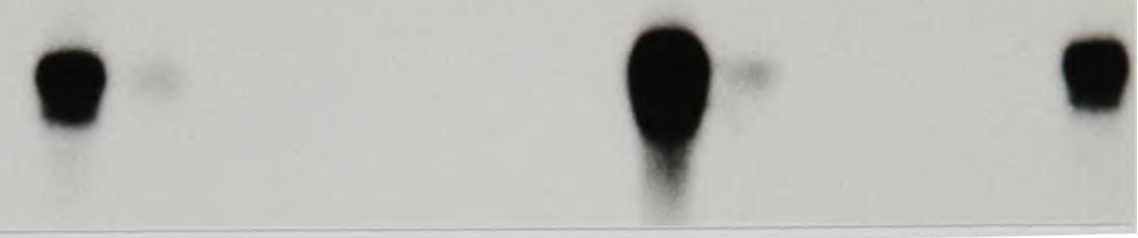

Figure 4.8 - RNase protection assay using the Epo-TAg riboprobe showing expression of the Epo-TAg transgene in seven different organs (abbreviations as in Figure 4.2) from acutely anaemic mice of three different lines. The Epo-TAg mRNA produces a band of $120 \mathrm{bp}$ and the endogenous Epo mRNA a band of 65bp. Kidney RNA from an acutely stimulated non-transgenic mouse is shown in the right hand lane for comparison. No transgene or endogenous gene expression is seen in any organ other than kidney or liver in this assay. The level of Epo-TAg and endogenous gene expression is much higher in kidney than in liver (in contrast to Epo$M)$.

\begin{tabular}{|l|c|c|c|c|c|c|c|c|c|c|}
\hline \multicolumn{1}{|c|}{ Line } & $\mathrm{K}$ & $\mathrm{L}$ & $\mathrm{B}$ & $\mathrm{H}$ & $\mathrm{Lu}$ & $\mathrm{S}$ & $\mathrm{G}$ & $\mathrm{T}$ & $\mathrm{O}$ & $\mathrm{U}$ \\
\hline 99.5 & - & - & - & - & - & - & $\mathrm{NE}$ & $\mathrm{NE}$ & - & - \\
\hline $134.3 \mathrm{HC}$ & + & $+^{*}$ & - & - & - & - & + & + & - & - \\
\hline 175.5 & + & - & + & - & - & - & + & + & - & - \\
\hline 176.5 & + & $-*$ & $+^{*}$ & - & - & - & + & + & $+^{*}$ & $+^{*}$ \\
\hline $188.1 \mathrm{LC}$ & + & + & - & - & - & - & $\mathrm{NE}$ & $\mathrm{NE}$ & + & $+^{*}$ \\
\hline $188.1 \mathrm{HC}$ & + & $-*$ & - & - & - & - & + & + & - & $+^{*}$ \\
\hline 188.3 & + & $+^{*}$ & - & - & - & - & + & + & - & $\mathrm{NE}$ \\
\hline Epo-TAg & + & + & + & - & - & - & + & + & - & - \\
\hline
\end{tabular}

Table 4.6 - Tissue distribution of Epo-M expression in transgenic mice treated with phenylhydrazine. The notation for organs is as in Table 4.1. + indicates correctly initiated transcripts, - indicates no correctly initiated transcripts. $*=$ additional incorrectly initiated transcripts. $N E=$ not examined .

In anaemic mice from all seven lines expressing the Epo-TAg transgene the highest level of expression was in the kidney. The level of Epo-TAg mRNA induced was different in each line and varied from less than $3 \%(134.3 \mathrm{HC})$ to approximately $30 \%(188.1 \mathrm{LC})$ of the abundance of endogenous Epo mRNA (Table 4.7 and Figure 4.8). In kidneys of nonanaemic animals Epo-TAg mRNA was below the limit of detection. A difference from the Epo-M lines was observed in hepatic expression. Whereas Epo-M lines overexpressed the transgene in liver at least ten fold, in 5 out of 6 Epo-TAg lines the ratio of hepatic to renal transgene expression resembled the ratio of expression for the endogenous gene (Table 4.7 and Figure 4.8). In the 176.5 line a high level of inducible transcription was observed in liver which was aberrantly initiated; this was also seen, but to a lesser extent, in liver in the 
188.1 HC and 134.3HC lines. In most other organs Epo-TAg mRNA was below the limit of detection except for the preputial gland (six lines), testis (six lines), ovary (two lines) and uterus (three lines).

\begin{tabular}{|l|c|c|c|}
\hline \multicolumn{1}{|c|}{ Line } & Copy no. & Kidney & Liver \\
\hline $134.3 \mathrm{HC}$ & 23 & $<3 \%$ & $<3 \%$ \\
\hline 175.5 & 13 & $4 \%$ & - \\
\hline 176.5 & 21 & $<3 \%$ & - \\
\hline $188.1 \mathrm{LC}$ & 2 & $27 \%$ & $<3 \%$ \\
\hline $188.1 \mathrm{HC}$ & 19 & $4 \%$ & - \\
\hline 188.3 & 2 & $21 \%$ & $<3 \%$ \\
\hline Epo-TAg & 1 & $100 \%$ & $<3 \%$ \\
\hline
\end{tabular}

Table 4.7 - Quantitation of Epo-TAg expression in the kidney and liver of acutely anaemic animals. The figures are for Epo-TAg transcripts, relative to the level of native Epo mRNA in the kidney of the same animal.

In Epo-TAgH mice the pattern of expression was closely similar to that of the endogenous Epo gene (Figure 4.8). Whilst inducible renal expression was substantially higher than in lines bearing the heterologously inserted transgenes, expression outside the liver and kidney was lower. Interestingly, although the expression of Epo- $\mathrm{TAgH}^{\mathrm{H}}$ and the native Epo gene were quantitatively very similar in the kidney, in the adult liver the expression of Epo$\mathrm{TAgH}^{\mathrm{H}}$ was consistently several-fold lower than that of the native Epo gene. As discussed in Chapter 6, compared to in situ hybridisation studies, the marker gene (based on detection of immunoreactive SV40 T antigen) was expressed at a lower level in hepatocytes compared to Ito cells.

\subsubsection{Foetal expression of Epo-TAg ${ }^{H}$}

A limited number of experiments were performed on foetal mice; in the foetal liver of heterozygous Epo-TAgH mice the transgene and native Epo gene were expressed at quantitatively similar levels, both at 13.5 and 14.5 days. The remainder of the foetus did not contain detectable Epo-TAgH $\mathrm{mRNA}$ or native Epo mRNA.

\subsubsection{Transient transfection studies}

In hepatoma cells, transfection studies demonstrated that a 96 bp sequence lying 3 ' to the Epo gene was both necessary and sufficient to confer hypoxic regulation on the Epo and $\alpha_{1}$-globin promoters (Beck et al. 1991, Semenza et al. 1991b, Pugh et al. 1991). Furthermore, in transgenic mice a DNase 1 hypersensitive site was demonstrated in the human Epo gene at this point (Semenza et al. 1991b). However, the difference between the renal expression in the 37.6LC line and the mice bearing the full Epo-M transgene demonstrate that sequence 
between $1.8 \mathrm{~kb}$ and $9 \mathrm{~kb} 5^{\prime}$ to the gene is important in determining renal expression of the gene. Thus this sequence might contain a kidney-specific hypoxically-inducible enhancer, or cis-acting sequence permitting expression of the gene in the kidney.

In order to assess whether the region lying between $0.4 \mathrm{~kb}$ and $9.0 \mathrm{~kb}$ was able to confer a hypoxic response on a heterologous promoter, 3 constructs were made which placed portions of this sequence in a plasmid containing alpha globin as reporter gene. As a positive control for hypoxic induction the construct pBS1-96 $\alpha$ was used (which contained 96 base pairs of the Epo 3' enhancer) and as a negative control pBS $\alpha$ was used, which was identical to the other test plasmids but contained only the alpha globin sequence (see Figure 3.1, p.58).

Transient transfection assays were performed in which each of these 5 constructs was cotransfected with ferritin-promoted growth hormone (FGH) as a control for transfection efficiency and RNA recovery. The cells used were hepatoma cells (HepG2), LLC-PK1 (a porcine proximal tubular cell line), M5 (a murine embryonic renal fibroblastoid line) and Cos7 cells (a simian fibroblast line). Cells were taken from a single pool and divided into five aliquots for transfection. Each aliquot of transfected cells was then divided for incubation at $1 \%$ oxygen or $21 \%$ oxygen. The level of $\alpha_{1}$-globin transcripts and FGH transcripts were measured by RNase protection. The ratio of hypoxic to normoxic expression of $\alpha_{1}$-globin was then calculated for each construct (corrected for FGH expression). The results are shown in Table 4.8. The three constructs bearing 5' Epo flanking sequence did not show hypoxic regulation under these circumstances in any of the cell types tested. In contrast, each of these cell types shows hypoxic regulation of pBS1-96 $\alpha$.

\begin{tabular}{|l|c|c|c|c|c|}
\cline { 2 - 6 } \multicolumn{1}{c|}{} & \multicolumn{5}{c|}{ Epo sequence cloned into $\mathrm{pBS} \alpha$} \\
\hline Cell line & $1-96$ & EcoR1/Sal1 & Xba1/Hinc2 & Kpn1/Cla1 & - \\
\hline LLC-PK1 & 4.2 & 1.2 & 1.1 & 1.0 & 1.2 \\
& 4.5 & 0.9 & 1.2 & 1.2 & - \\
\hline Cos7 & 2.6 & 1.5 & 1.7 & 1.4 & - \\
& 1.4 & 0.9 & 0.9 & 0.9 & 1.0 \\
\hline M5 & 4.2 & 1.3 & 1.3 & 1.2 & 1.6 \\
& 1.7 & 1.0 & 1.0 & 1.0 & 0.9 \\
\hline HepG2 & 15.9 & 0.8 & 0.9 & 1.0 & 1.5 \\
\hline
\end{tabular}

Table 4.8 - Results of transient transfection experiments using 5'flanking sequence linked to $\alpha$ globin. The figure given is for the hypoxic:normoxic ratio of reporter gene expression (corrected for expression of the co-transfected control plasmid). Each line represents an experiment on a single pool of cells. The positive control was pBS1-96 $\alpha$ which contains the Epo 3' enhancer. The negative control, pBS $\alpha$, contains no Epo sequence. The other 3 constructs contained different portions of the $8.5 \mathrm{~kb} 5^{\prime}$ to the Xbal site of the mouse Epo gene. No evidence of a hypoxic response dependent on the presence of Epo 5'flanking sequence was observed. 


\subsection{DISCUSSION}

The first aim of these experiments was to define the range of organs expressing Epo in normal mice. Ten organs were examined. As in previous studies, both of mice and other animals, a low level of expression was found in the kidney of unstimulated mice. In kidney and liver there was high-level induction of Epo expression in response to anaemia, and the level of Epo mRNA in the liver (per $\mu \mathrm{g}$ of RNA) was much less than that in the liver. In contrast to a previous report (Semenza et al 1990), evidence of the use of other transcription start sites for erythropoietin was not observed.

The first new observation in the mouse is that the Epo gene is expressed in the brain and the testis. These are in agreement with recent observations in the rat (Tan et al. 1992). As in the rat, the level of expression was induced somewhat (approximately threefold) in response to anaemia. In addition two new sites of Epo expression have been identified for the first time in any species; the preputial gland and the ovary. In the former, but probably not in the latter, anaemia resulted in an increased level of expression.

As has been described in the rat (Tan et al. 1992) the level of Epo mRNA seen in some sites outside the liver and kidney (in particular the testis and preputial gland) is not dissimilar from that seen in the kidney in unstimulated animals. However, it is not known if this mRNA is translated, nor is it known whether Epo produced locally in these organs would reach the bloodstream. Certainly in response to anaemia the level of Epo mRNA in the liver and kidney is much higher than that observed elsewhere.

The next aim of these experiments was to identify sequence which would direct fully regulated expression of a marker gene in transgenic animals. It can be concluded from the results of the Epo-M experiments that the $16.5 \mathrm{~kb}$ from the Epo locus can direct hypoxicallyregulated gene expression in the liver and kidney, and also in the brain, testis and preputial gland. However, these transgenes were over-expressed in liver compared to kidney. This suggests that a negative element normally operating in the liver lies outside the sequence used in the transgene; this result is entirely consistent with the suggested structure of the human gene based on Semenza's transgenic experiments (Semenza et al. 1989, Semenza et al. 1990, Semenza et al. 1991a). The tendency to over-expression in some other sites suggests that distant negative regulatory elements are not only important in the liver but also operate in other tissues. 
Further evidence for the importance of distal sequence is the observation that Epo-M transgenes did not show copy-number dependence, and that the renal expression per copy was lower than that of the endogenous gene. It is therefore likely that more distant elements involved in control of Epo gene expression; whether there is a distinct locus control region such as those identified for $\beta$ globin (Crossley and Orkin 1993) and a few other genes remains to be determined. Cosmid clones have been isolated which extend $5^{\prime}$ and $3^{\prime}$ to the $\lambda 3$ a clone (Appendix 3), and should allow identification of distant cis-acting elements in further transgenic experiments.

In contrast to Epo-M, the Epo-TAg transgenes which included identical Epo sequence did not in general show over-expression in the liver. This was likely to be due to the insertion of the SV40 sequence; further support for this hypothesis is that in the Epo-TAgH mice, the recombinant gene is under-expressed in the liver (particularly in hepatocytes, see Chapter 6), whereas in the kidney the gene appears to be expressed at the same level as the native gene. There are several possible explanations for this effect of the SV40 sequence; one is that it distances important control sequence for hepatic expression of the gene (eg the $3^{\prime}$ enhancer) from the promoter. Another is that transcription factors interact with the SV40 sequence.

The failure of a truncated Epo-M transgene (37.6LC) to express in the kidney should be interpreted cautiously, as position effects could certainly have influenced the pattern of expression. However, it suggests that in the mouse an important positive regulatory element for expression in the kidney lies between $1.8 \mathrm{~kb}$ and $9 \mathrm{~kb} 5$ ' to the gene. Further, Semenza's tgEPO4 transgene was expressed at a high level in many tissues, in contrast to 37.6LC which was restricted to liver and brain. Taking these findings together it is likely that a negative regulatory element capable of operating widely lies between the equivalent of $0.7 \mathrm{~kb} 5^{\prime}$ to the human gene and $1.8 \mathrm{~kb} 5^{\prime}$ to the mouse gene (see Figure 4.9). Such a region should be conserved between mouse and man, and comparison of the sequence in this region between mouse and man has shown three regions with greater than $60 \%$ homology between mouse and man, of between 108 and 131 bp (D,E and F in Galson et al. 1993; and see Figure 1.1, p.9). The observation that expression of the truncated transgene in 37.6LC was hypoxically regulated in the liver is consistent with Semenza's observation that tgEPO4 expression in the liver was regulated by hypoxia (Semenza et al. 1989). In addition, expression in the brain of the 37.6LC mice was also hypoxically induced. Thus elements 
within the gene itself, approximately $1.8 \mathrm{~kb}$ of 5 ' flanking sequence and $3.5 \mathrm{~kb}$ of 3 ' flanking sequence are capable of conferring hypoxic regulation in the liver and brain in chromatin. This region includes the 3 ' enhancer region used in the experiments described in Chapter 3.

\section{HUMAN}

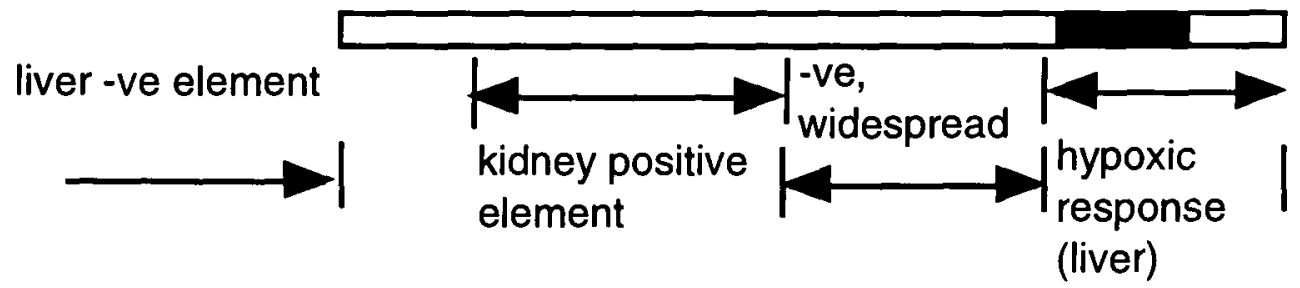

MOUSE

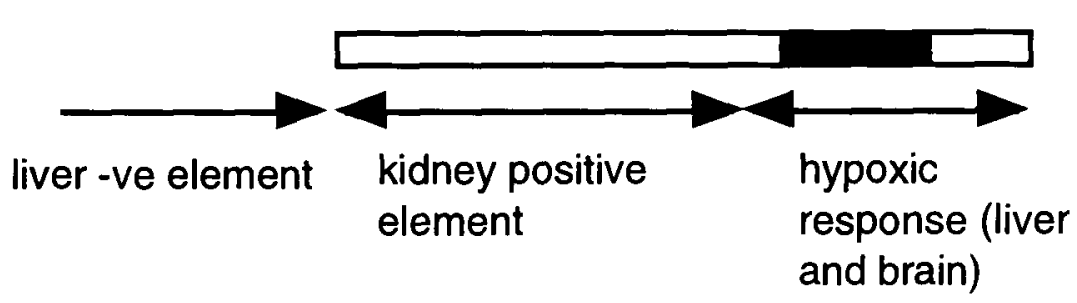

\section{COMBINED}

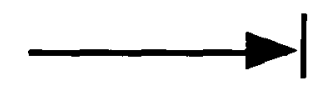

liver -ve element $\left(>16 \mathrm{~kb}^{\star}\right)$

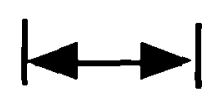

kidney +ve element $\left(>5 \mathrm{~kb}^{\star},<9 \mathrm{~kb}\right)$

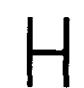

-ve, widespread $\left(>0.4 \mathrm{~kb}^{*},<1.8 \mathrm{~kb}\right)$

Figure 4.9 - Comparison of information obtained from Semenza's experiments using human Epo transgenes and the current experiments. The Epo gene is shown as a solid box. The extent of DNA is $22 \mathrm{~kb}$ (human) and $16.5 \mathrm{~kb}$ (mouse). The data are compatible, and combining the two gives improved precision regarding the position of regulatory sequences. In the combined illustration the positions indicated are distances 5' to the transcription start site; * indicating a distance derived from the human experiments.

In terms of further analysis of cis-acting sequence, the transient transfection studies described here are of limited value. They did not demonstrate any hypoxic induction when placed in the context of the human $\alpha_{1}$-globin gene, in contrast to experiments with the 3 ' enhancer. This strongly suggests that the element (or elements) in this 5 ' region which allow expression in the kidney in transgenic animals do not behave in the same fashion as the 3 ' enhancer. It is possible that this $5^{\prime}$ sequence, rather than being a hypoxia-responsive element, is permissive in nature and its presence is necessary to allow the operation of the $3^{\prime}$ enhancer in the kidney. Alternatively, the 5 ' sequence could include hypoxic response element(s) which were cell-specific or operated in a different range from the stimulus found to operate effectively on the $3^{\prime}$ enhancer ( $1 \%$ oxygen in a tissue culture incubator). It is also possible that in vivo the renal response may not be to hypoxia in the Epo-producing cells; hypoxia might be sensed by one cell-type, which then produced a substance acting on another cell. Another possible explanation would be that the relatively large sequences employed in these transfection experiments contained hypoxia-responsive elements, but also included suppressive elements. 
An interesting observation is the number of tissues which are capable of expressing the Epo gene at a significant level. This contrasts with the effect of renal disease and with the effects of bilateral nephrectomy in experimental animals. Furthermore, it suggests that either Epo can be expressed by a wide range of cell-types, or that it is expressed by a cell that is present in many organs. Previous in situ studies in the kidney demonstrate that a high proportion of the cortical (and outer medullary) interstitial cells have the capacity for Epo gene expression; if these cells are of a previously recognised type they would be fibroblastoid, endothelial, macrophages or the antigen-presenting dendritic cells. In the liver in situ hybridisation studies suggest that two populations express the gene; one is the hepatocytes. Thus there is evidence that more than one cell-type, and several tissues have the ability to express the gene. Unlike the liver, the brain shows striking regional heterogeneity in terms of structure. The possibility that the ability to produce Epo would be restricted to a particular region of the brain was therefore tested. However, mRNA analysis of portions of brain from anaemic rats provide evidence that the ability to express the gene is in fact distributed evenly in the brain. Since these experiments were performed, cell culture studies suggest that astrocytes cultured from foetal rat brain express the Epo gene (Masuda et al. 1994). In addition these cells showed increased expression of Epo in response to hypoxia.

The expression of native Epo, the Epo-M transgene and Epo-TAg transgene in most tissues was clearly modulated by hypoxia. The observations in this chapter are concerned with transgenic DNA which is subject to the influence of chromatin structure and will include multiple cis-acting elements. This complements the transient transfection studies described in Chapter 3, in which transient transfection of the Epo 3' enhancer was used to identify a widespread oxygen-sensing mechanism in cultured cells. The hypoxic regulation of the native Epo gene and Epo transgenes in mice is likely to involve elements other than the 3' enhancer. However, the transgenic experiments clearly demonstrate that several tissues are able to modulate gene expression in response to a physiologically relevant stimulus (anaemia).

The polycythemia in the 198.3 Epo-M line and in the 176.5 and 188.1HC Epo-TAg lines is of some interest. In both cases the difference from control mice was much less marked at the time of weaning than in older animals. In each case it is likely that the excess Epo was due to incompletely regulated or unregulated transcripts in tissues other than the liver and kidney. An important point is that transcripts from the Epo-TAg gene run through the SV40 
polyA signals into Epo coding sequence (see Chapter 8). Thus expression of the Epo-TAg transgene can lead to the production of Epo without advocating expansion of the Epoproducing cell population. That the polycythaemia in 198.3, 176.5 and $188.1 \mathrm{HC}$ occurred at about the time of sexual maturity suggests that it may be sex-hormone dependent. This was not further investigated.

Finally, the faithful expression of the Epo-TAg gene, as assessed by tissue distribution and induction by anaemia and hypoxia, provides cogent evidence for the use of SV40 T antigen as an appropriate marker for Epo gene expression. In the case of the Epo-TAgH mice this approach is further justified by the presence not only of the $16.5 \mathrm{~kb}$ cloned from the Epo locus, but also the presence of all possible regulatory elements. 


\section{Identification of the renal Epo-producing cells}

\subsection{INTRODUCTION}

This chapter describes the localisation of SV40 T Ag at a cellular level in the kidney of Epo-TAg mice. Mice with integration of the transgene both at homologous and heterologous sites were studied. The aim of these experiments was to identify and characterise the Epoproducing cells in the kidney. This was achieved by double-labelling studies and by immunoelectron microscopy.

As discussed in Chapter 1, the localisation of Epo-production within the kidney has been controversial. Identifying the cell-type involved would be an important step in understanding the control of Epo production in health and disease. In situ hybridisation for Epo mRNA has in several studies suggested that Epo is produced by an uncharacterised interstitial cell population (Koury et al. 1988b; Lacombe et al. 1988; Kurtz et al. 1989). On the other hand, another study has suggested localisation of Epo and Epo mRNA in the tubular cells (Maxwell et al. 1990).

Unfortunately, it is difficult to combine in situ hybridisation with immunolabelling. The strategy used in this chapter has several advantages. Firstly, it is relatively easy to combine localisation of the stable nuclear marker protein, SV40 T Ag, with labelling of cytoplasmic or cell-surface antigens. Secondly the background staining was very low, and the sensitivity appeared to be high; this is likely to be due to the progressive accumulation of the marker protein in the nucleus. Thirdly, it was possible to perform immunoelectron microscopy allowing ultrastructural identification of these cells.

However, there are two important caveats. Firstly, the information obtained is indirect evidence for Epo gene expression. This has been addressed by comparison with previous in situ studies. Secondly, it is possible that expression of SV40 T Ag (a transcriptionally active protein) might alter the behaviour and number of Epo-producing cells. This has been minimised by performing acute studies on young animals. 


\subsection{METHODS}

\subsubsection{Animals}

The animals used in these experiments were Epo-TAg transgenic mice aged 6 weeks to three months. Non-transgenic controls were from the same colony. In order to induce expression of the Epo-TAg gene in the kidney, mice were given phenylhydrazine to induce an acute haemolysis or exposed to hypobaric hypoxia $(7200 \mathrm{~m})$.

\subsubsection{Immunolabelling for light microscopy}

The principle technique employed in this chapter was the localisation of various antigens using indirect immunolabelling, at the light microscopic and ultrastructural level. A range of polyclonal and monoclonal antibodies (mAbs) was employed, all of which had previously determined specificities. For each of these antibodies, preliminary experiments were performed to determine suitable dilutions of the primary antibody, a suitable detection system and the distribution of the antigen in the tissue sections. These preliminary experiments were performed on acetone-fixed cryosections, in which preservation of antigenicity appeared optimal.

Central to this study was the use of antibodies to SV40 T Ag. Cytospins of Cos7 cells, which express SV40 T Ag, were fixed and stained in parallel with the kidney sections to serve as positive controls. Kidney sections from non-transgenic mice served as negative controls, in addition to technical controls with appropriate substitution for the antibody directed against SV40 T Ag. The various antibodies used and dilutions are given in Table 2.3 (p.51). Although mouse mAbs to various antigens were tested, the application of antimouse Igs always resulted in a high level of non-specific staining. The antibodies employed were therefore generally either rat monoclonals or rabbit polyclonal antisera. However, it was found to be possible to employ mouse mAbs to SV40 T Ag conjugated to biotin, followed by avidin-conjugated horseradish peroxidase. In order to achieve a low background it was found to be necessary to block endogenous biotin by the prior application of avidin followed by biotin.

Immunolabelling of tissue sections usually involves a compromise between morphology (which improves with more effective fixation) and antigen preservation (which tends to be reduced by more effective fixation). Under some circumstances an antigen can be recovered following fixation by the use of microwaves or controlled protease digestion. Furthermore, the optimal compromise is epitope-specific. For light microscopy, it was found to be possible to combine SV40 T Ag staining with perfusion-fixation using aldehyde-based fixatives 
followed by paraffin-embedding or cryosectioning of sucrose-balanced material. In paraffin sections, labelling for SV40 T Ag was only possible with the polyclonal antiserum and did require recovery of antigen either by protease digestion or microwave treatment. In sucrosebalanced material the biotinylated mAbs to SV40 T Ag could also be used. Neither the fixative employed nor the buffer appeared to be critical. The only fixation protocol following which the SV40 T Ag was not recoverable was following fixation with $2 \%$ glutaraldehyde.

The cytoplasmic and cell-surface antigens were generally less robust. It was possible to detect ecto-5'-nucleotidase (5'NT) and ER-TR7 in perfusion-fixed, paraffin-embedded sections or perfusion-fixed, sucrose-balanced frozen sections. Following paraffinembedding, this required protease digestion, and neither antigen was revealed by microwave treatment. CD45, Factor VIII rAg, and PECAM/CD31 and the F4/80 antigen could be detected in perfusion-fixed, sucrose-balanced frozen sections. Thy 1 and Class II MHC (Ia) could only be detected in acetone-fixed frozen sections.

Following optimisation of staining methods in single-labelling studies, double-labelling studies were performed. Antibodies derived from different species were used to detect SV40 T Ag and cell-surface antigens. Different enzymes were then used to localise the antibodies (peroxidase for SV40 T Ag and alkaline phosphatase for cell-surface antigens). Appropriate controls for non-specific staining were performed in parallel on adjacent tissue sections in all double-labelling experiments.

One potential concern is that cells might fail to label for an antigen if the sensitivity was reduced by fixation. Consequently, although the double-labelling results presented were performed in the material allowing the best morphological preservation compatible with antigen detection, similar experiments were also performed in acetone-fixed cryosections. The results obtained were essentially identical.

\subsubsection{Morphometric assessment}

This was performed with a $40 \mathrm{x}$ objective on a Leitz microscope. The method used was to count the total number of interstitial nuclei in an area defined by an eyepiece graticule of dimensions $1 \mathrm{~cm} \times 1 \mathrm{~cm}$. This corresponded to $250 \mu \mathrm{m} \times 250 \mu \mathrm{m}$ of the section. The tissue sections to be compared were cut from the blocks on the same microtome to ensure that they were the same thickness, and were then counterstained with PAS. The total number of interstitial nuclei was counted in at least nine fields in each kidney; the areas examined forming three strips extending from the capsule into the outer medulla. In order to establish 
the distribution of cells positive for SV40 T Ag the sections were first labelled for SV40 T $\mathrm{Ag}$ using nickel-enhanced $\mathrm{DAB}$ and then counterstained with PAS. The total number of interstitial cells, and the number of cells labelled for SV40 T Ag was then counted in three strips as above.

\subsubsection{Immunoelectron microscopy}

The immunoelectron microscopy described in this chapter was performed by Dr D. Ferguson. Plastic-embedded sections were indirectly labelled with gold conjugates. The amount of antigen available for localisation in such sections is much reduced compared to the methods used for the light microscopy experiments, because the sections are essentially impermeable. Although several antibodies were tested, including MEC13.3 and ER-TR7, it only proved possible to detect SV40 T Ag and 5'NT. The method used is described in Chapter 2. Doublelabelling of grids was attempted, by first labelling one side for SV40 T Ag and then labelling the other for 5'NT using a different size gold conjugate. Unfortunately, it proved very hard to keep one side of the grid dry, because of the Tween in the solutions. Consequently, this method was not generally used and labelling of the antigens was performed on adjacent sections, in which it was straightforward to identify individual cells.

\subsection{RESULTS}

\subsubsection{Demonstration of SV40 T Ag in transgenic kidney}

Initial studies were performed on acetone-fixed frozen sections from the kidneys of EpoTAg mice treated with phenylhydrazine. In these cryostat sections of kidney, a number of positive nuclei were observed, which were located in the interstitium of the deep cortex and of the outer medulla. No staining was observed in kidneys from non-transgenic animals.

For studies of the cellular distribution of SV40 T Ag the experiments were performed in perfusion-fixed paraffin-embedded kidney sections using the polyclonal antiserum. Heterozygous Epo-TAg ${ }^{\mathrm{H}}$ mice were used for these experiments, since renal expression of the transgene was higher than in the other lines. Furthermore, as discussed in Chapter 4, in this line the marker gene is under the control of all cis-elements at the Epo locus. The same cellular pattern of expression in the renal interstitium was, however, observed in mice bearing heterologously inserted transgenes (188.1LC, 188.3, 188.1HC and 176.5). In these lines the lower level of Epo-TAg expression appeared to result in detection of SV40 T Ag in fewer cells at the same level of haematocrit. 


\subsubsection{Immunolocalization of Epo-TAg expression}

The Epo-TAg ${ }^{\mathrm{H}}$ animals studied were between 1 and 3 months of age and were either unstimulated or stimulated acutely with phenylhydrazine. No abnormalities were observed in the renal tubules or glomeruli. Since the expression of SV40 T Ag in the kidney might have altered the number of cells, this was assessed carefully. However, the renal interstitium appeared normal, in particular the interstitial cell density was similar between non-transgenic and transgenic mice. These quantitative assessments are given in Table 5.1.

\begin{tabular}{|l|c|c|c|c|}
\cline { 2 - 5 } \multicolumn{1}{c|}{} & $\begin{array}{c}\text { outer } \\
\text { cortex }\end{array}$ & $\begin{array}{c}\text { middle } \\
\text { cortex }\end{array}$ & $\begin{array}{c}\text { inner } \\
\text { cortex }\end{array}$ & $\begin{array}{c}\text { medulla } \\
\text { outer stripe }\end{array}$ \\
\hline Transgenic & $1296+/-170$ & $1397+/-139$ & $1430+/-373$ & $1712+/-341$ \\
\hline Controls & $1296+/-106$ & $1362+/-90$ & $1408+/-182$ & $1752+/-157$ \\
\hline
\end{tabular}

Table 5.1 - Comparison of the total number of interstitial cell nuclei per mm ${ }^{2}$ in two Epo-TAg ${ }^{H}$ heterozygotes and two non-transgenic littermates. Mean $+/-s d$ is given. The counts were performed on three strips of width $250 \mu \mathrm{m}$ running perpendicular to the capsule from each animal. No significant difference was found.

In anaemic Epo-TAg mice, immunoreactive SV40 $\mathrm{T} \mathrm{Ag}$ was induced in a population of renal interstitial cells (Figures 5.1 and 5.2). Staining was confined to the nucleus (Figure 5.3A). This pattern of expression was not only observed in mice with anaemia induced by phenylhydrazine; it was also seen in iron deficiency anaemia, the constitutively anaemic Epo-TAg ${ }^{\mathrm{H}}$ homozygotes, crosses with Nan mice (which have an autosomal dominant haemolytic anaemia), and in animals exposed to hypobaric hypoxia. No staining was seen in kidneys from non-transgenic mice.

All cells expressing SV40 T Ag were within the renal interstitium. No glomerular or tubular staining was observed in any animal. In kidneys from unstimulated animals a few cells were positive for SV40 T Ag, and these were located in the deep cortex. In kidneys from anaemic animals an increased number of cells were positive in the deep cortex, but with more severe stimulation recruitment was observed, with cells expressing SV40 T Ag being observed throughout the cortex and outer stripe of the outer medulla (Figures 5.1, 5.2 and 5.3). Some cells between thick ascending limbs of Henle's loops in the outer portion of the inner stripe of the outer medulla also expressed SV40 T Ag. In these regions the cells appeared to be located distant from the vascular bundles. The cell density throughout the cortex was not uniform and the cells often appeared in small clusters. 


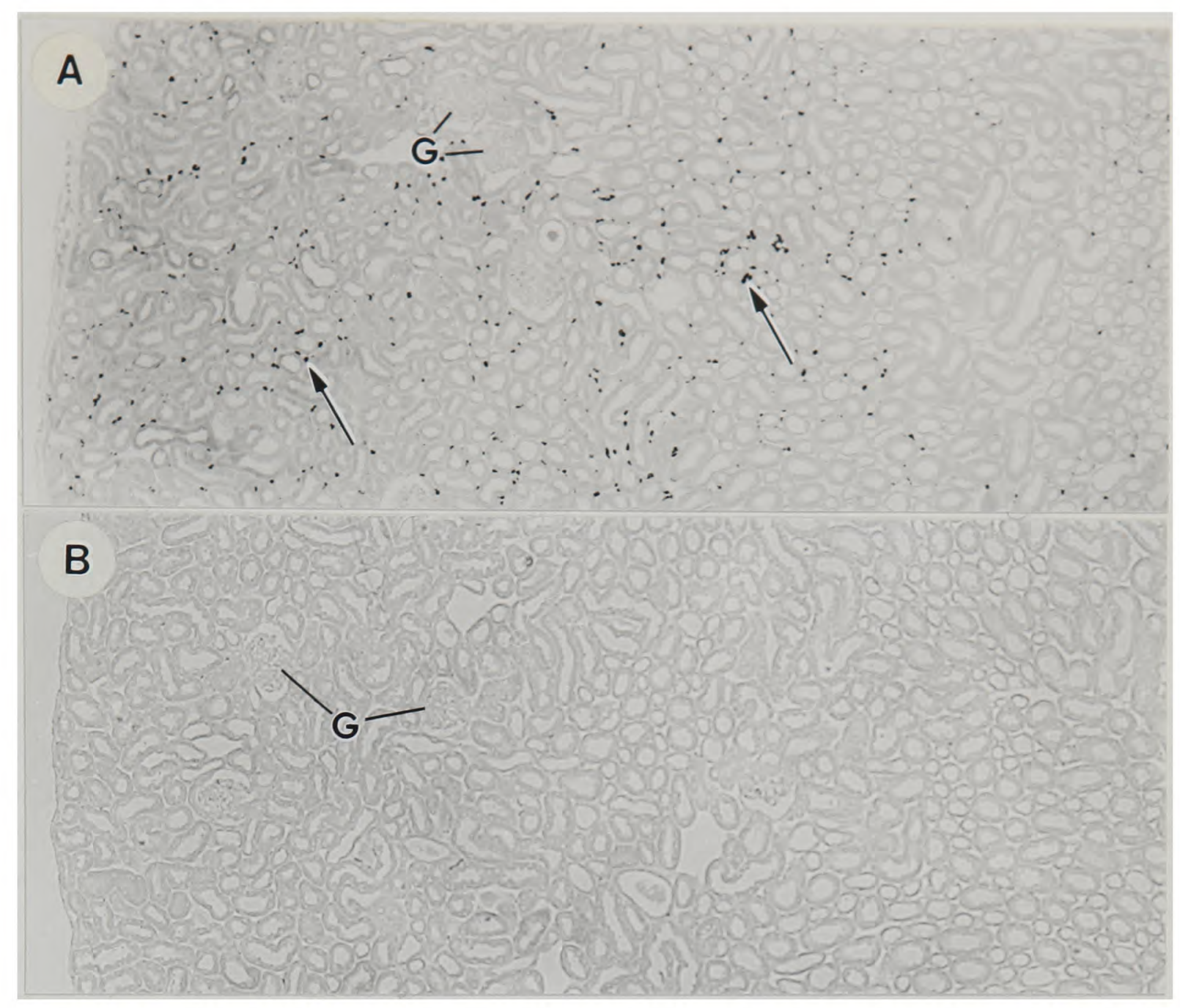

Figure 5.1 - A. Section of a kidney from an acutely anaemc Epo-TA ${ }^{H}$ mouse (haematocrit $17 \%)$, showing the distribution of SV4O T Ag positive nuclei. The transverse section shows the full thickness of the cortex and the outer stripe of the outer medulla, and a part of the inner stripe of the outer medulla. Glomeruli $(G)$ are identifiable in the cortex. Nuclei positive for $\mathrm{T} \mathrm{Ag}$ are seen as black dots lying between the tubules, and are visible through the entire depth of the cortex and in the outer stripe of the outer medulla. Magnification $\times 50$. $B$. Control section from the same area of the kidney shown in A. The antiserum to SV4O T Ag was replaced with normal rabbit serum. The level of background staining is low, and the interstitial nuclear staining seen in A is specific for TAg. Magnification $x 50$.

In a single experiment the response to cobaltous chloride was tested. Again, only interstitial cells contained SV40 T Ag. Interestingly, the distribution of positive cells was similar to that observed in hypoxic or anaemic animals, with cells containing SV40 T Ag being predominantly located in the deep cortex. This suggests that the effect of cobalt may not be independent of the effect of local hypoxia. This would be consistent with a previous study in rats which demonstrated that the effect of cobalt could be suppressed by hyperbaric hyperoxia (Necas and Neuwirt 1971).

In order to assess the recruitment pattern in anaemia and to compare the number of cells containing SV40 T Ag with previous quantitative assessments from in situ hybridisation for Epo mRNA, quantitative studies were performed. The data obtained are given in Figure 5.2. In sections from severely anaemic mice, approximately $40 \%$ of all interstitial cells stained positively in the deep cortical regions. These proportions are similar to the estimates from in situ studies (Koury et al. 1989; Lacombe et al. 1988). 


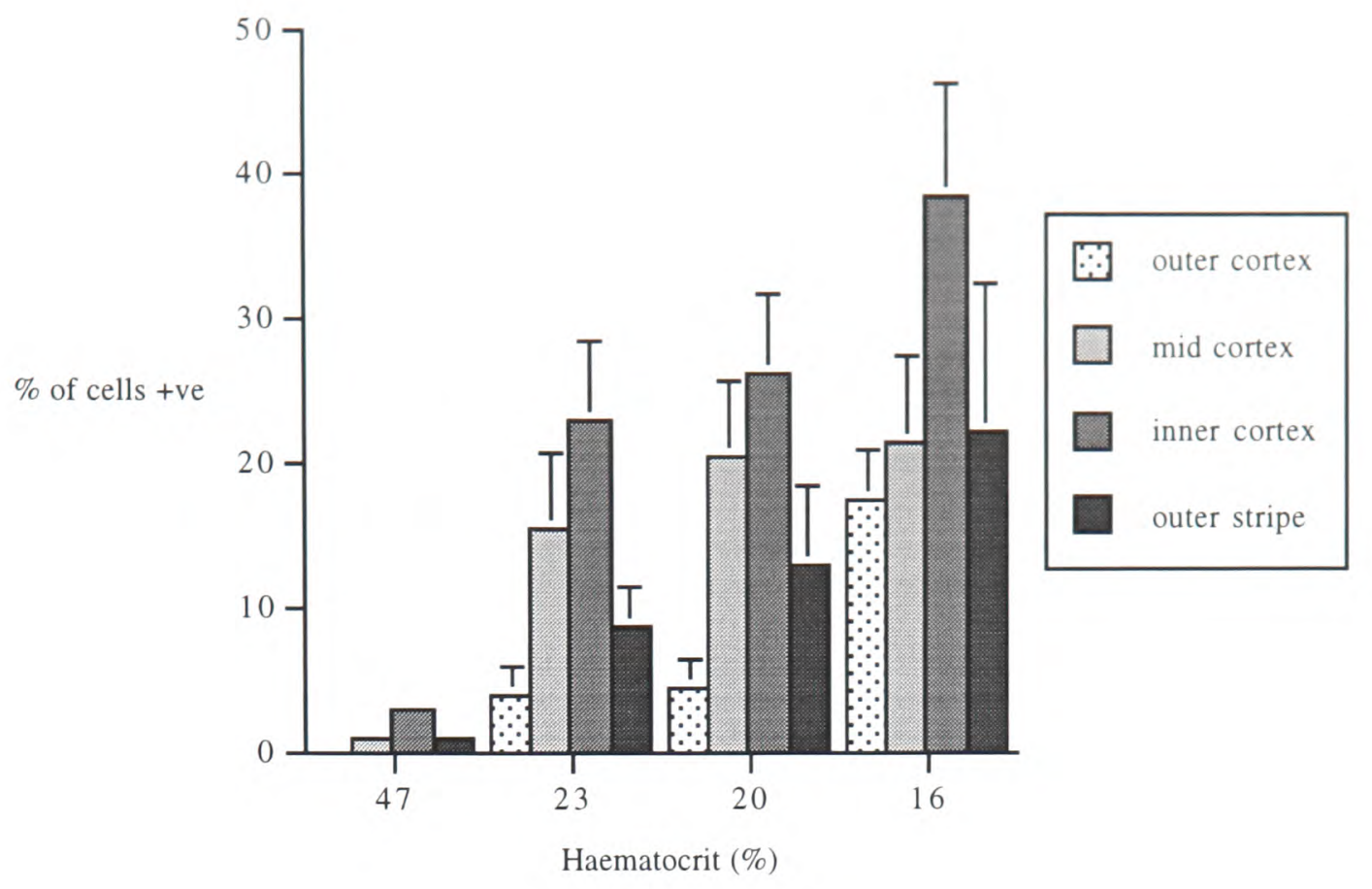

Figure 5.2 - Histogram showing distribution of Epo-producing cells in the mouse kidney and recruitment in anaemia. Data from four transgenic mice (Epo-TA $g^{H}$ heterozygotes) are shown, three of which were rendered anaemic to different degrees with phenylhydrazine. The kidneys were perfusion-fixed and paraffin-embedded. In each mouse three strips of width $250 \mu m$ running perpendicular to the capsule were assessed, which traversed the full thickness of the cortex and outer stripe of the outer medulla. The cortex was subdivided into thirds on the basis of depth. The percentage of interstitial nuclei positive for SV4O TAg is shown (mean of three values). Error bars show standard deviation. In the unstimulated mouse (haematocrit 47\%) there were too few positive cells for this method to be representative. In this kidney all the positive cells in a transverse section of the kidney were counted, and the total number of interstitial cells was estimated from the data in Table 5.1.

In these paraffin-embedded sections it was not possible to identify which interstitial celltype was expressing the marker on morphological grounds. The cells bearing SV40 T Ag had nuclei of variable shape and using conventional counterstaining the cytoplasm was rather poorly visualized. Further characterisation was performed using double labelling and immunoelectron microscopy.

\subsubsection{Double-labelling}

The antibodies used to characterise interstitial cells in double labelling experiments are given in Table 5.2. 


\begin{tabular}{|l|l|c|l|}
\hline \multicolumn{1}{|c|}{ Antigen } & \multicolumn{1}{c|}{ Antibody } & Dilution & \multicolumn{1}{c|}{ Labelling in interstium } \\
\hline F4/80 Ag & Rabbit a/s & $1: 1000$ & Macrophages \\
\hline Ecto-5'-nucleotidase & Rabbit a/s & $1: 1000$ & Cortical fibroblast-like cells \\
\hline Factor VIII rAg & Rabbit a/s & $1: 200$ & Endothelium (large vessel) \\
\hline CD45 & M1/9.3.4.HL2 & neat & Leucocytes \\
\hline Class II MHC (Ia) & B21-2 & neat & Leucocyte subset \\
\hline Thy 1 & YBM 29.2.1 TG6 & neat & Leucocyte subset \\
\hline Fibroblast marker & ER-TR7 & $1: 25$ & Fibroblast-like cells, connective tissue \\
\hline PECAM/CD31 & MEC 13.3 & neat & Endothelium \\
\hline
\end{tabular}

Table 5.2 - Antibodies used to identify renal cell populations. Rabbit antisera were detected with swine anti-rabbit antibodies conjugated to alkaline phosphatase. The mAbs are all of rat origin and were detected with swine anti-rat Fab fragments. The Thy 1 and Class II MHC epitopes were not preserved in perfusion-fixed sections, and for these antibodies acetonefixed frozen sections were used. ER-TR7 was from Biogenesis (Bournemouth), Rabbit antihuman factor VIII $\mathrm{rAg}$ was from DAKO. The antisera to F4/80 and 5'NT were gifts from $\mathrm{Dr}$ $P$. Dri and Prof. B. Kaissling respectively. The antibody MEC13.3 was a gift from $\mathrm{Dr} A$. Vecchi.

First, colocalization of SV40 T Ag with endothelial markers was studied. Rabbit antiserum to human Factor VIII-related antigen did not colocalize with the antibody to SV40 T Ag. Although intense staining of the endothelium of large vessels was observed, capillary staining with this antiserum was much less intense leaving some doubt as to the security of this negative result. Therefore further studies were performed with a rat anti-mouse PECAM/ CD31 mAb (MEC13.3). This antibody reacted strongly with capillary endothelium in the interstitium. Endothelial nuclei were clearly demonstrated and all were negative for SV40 $\mathrm{T} \mathrm{Ag}$ (Figure 5.3B). Likewise no cell expressing SV40 T Ag expressed PECAM/CD31, indicating that these cells were not endothelial. 


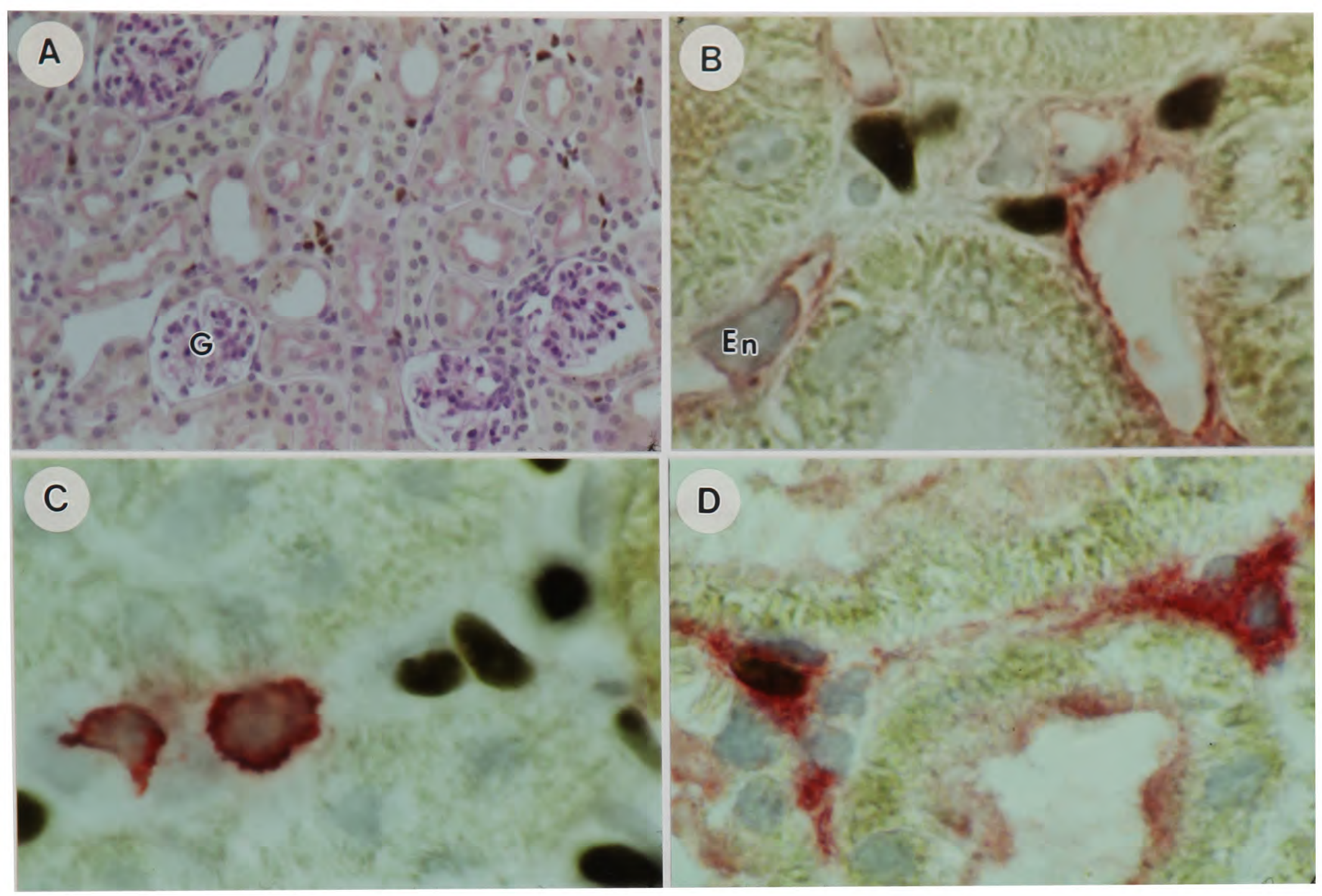

Figure 5.3 - A. Kidney section from a stimulated Epo-TA ${ }^{H}$ heterozygote immunostained for SV40 T Ag and counterstained with PAS, showing a region of deep cortex. Four glomeruli $(G)$ are seen. Positive nuclei (brown) lie between the tubules. Magnification x 125.

B. Double staining for SV4O T Ag and PECAM/CD31. Four nuclei positive for SV40 T Ag (black) are seen to lie between four tubules. The endothelium of four capillaries is positive for PECAM/CD31 (pink). An endothelial nucleus is seen (En) which is not positive for SV40 TAg. Magnification $x 900$.

C. Double staining for SV4O TAg and CD45. Several nuclei positive for SV4O TAg are seen (black), and two CD45 positive cells are shown (pink), which are negative for SV4O T Ag. Magnification $\times 900$.

D. Double staining for SV4O T Ag and 5'NT. Two interstitial cells positive for 5'NT are seen (red staining) lying adjacent to a proximal tubule. One of these is also positive for SV4O TAg (black nucleus). Several other interstitial cells are negative for both markers. There is some weak 5'NT staining of the brush border of the tubular cells. Magnification $x 900$.

The non-endothelial cell populations within the renal interstitium include several leucocyte populations: macrophages, lymphocytes and a cell population of dendritic form which is of bone marrow origin (Hart and Fabre 1981; Kaissling and Le Hir 1994). All of these cells bear leukocyte common antigen (CD45). A rat anti-mouse CD45 mAb was used to determine whether there was SV40 T Ag in CD45 bearing cells. Although a substantial interstitial population was CD45 positive, none of the SV40 T Ag expressing cells stained with this $\mathrm{mAb}$ (Figure 5.3C). Sections were also stained with rabbit polyclonal antiserum to the macrophage $F 4 / 80$ antigen, and with the original rat $\mathrm{mAb} F 4 / 80$. Interstitial cells expressing F4/80 antigen were observed in both cortex and medulla, but, as is consistent with the studies of CD45 expression, these cells were distinct from those expressing SV40 T Ag. 
Interestingly, a significant proportion of SV40 T Ag-expressing cells, in the region of 5\%, appeared to be closely apposed to cells expressing CD45 and F4/80 antigen. Double staining was also performed for SV40 T Ag with mAbs to Class II MHC and to Thy 1. Rather fewer interstitial cells expressed Class II MHC or Thy 1 than had been identified with the CD45 $\mathrm{mAb}$, and none of these cells was positive for SV40 T Ag.

The renal interstitium also contains cells which have been classified as fibroblasts (Type 1 interstitial cells) on morphological grounds (Lemley and Kriz 1991; Kaissling and Le Hir 1994). Recent studies have characterised the expression of the ectoenzyme 5'NT in the renal interstitium using histochemical and immunohistochemical techniques (Dawson et al. 1989; Le Hir and Kaissling 1989). These studies indicate that this antigen is a marker for the fibroblasts (Type 1 interstitial cells). It is also expressed by lymphatic endothelium, but not by capillary endothelium. As described previously in the rat, the 5'NT antiserum labelled the brush border of proximal tubules, with particular intensity in the S3 portion lying in the outer medulla, and also labelled glomeruli (Figure 5.4).

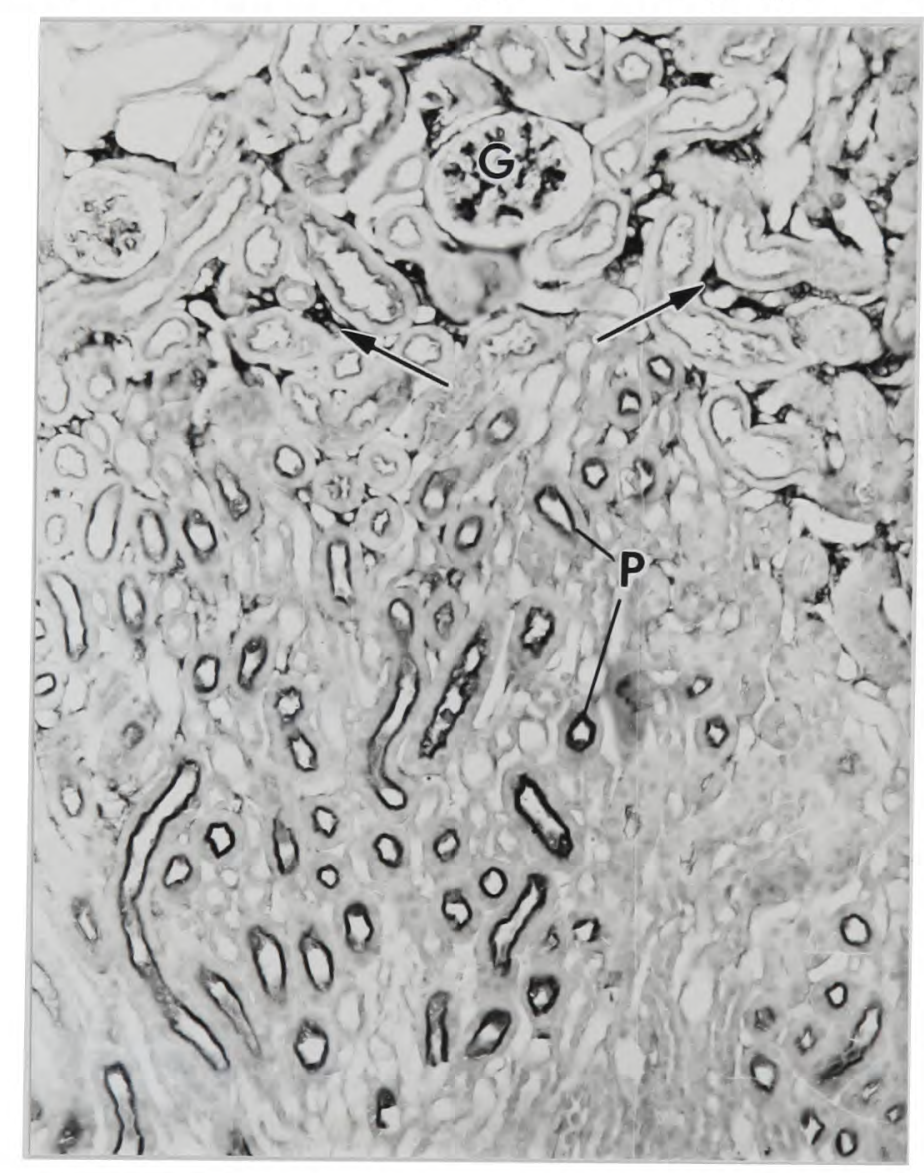

Figure 5.4 - Section through cortex and outer medulla of kidney immunostained for $5^{\prime} N T$. Note positive staining in the glomerulus $(G)$, proximal tubules $(P)$ and interstitial cells (arrows). Wax embedded, peroxidase stained. Magnification $x 50$.

Interstitial staining with 5'NT was essentially confined to the cortex and was most intense in the deep cortex, where SV40 $\mathrm{T} \mathrm{Ag}$ positive cells were also most frequent. In these regions the great majority of cells containing nuclear SV40 T Ag labelled for 5'NT, identifying them as the fibroblasts (Type 1 interstitial cells). However, in severely anaemic animals, although substantial numbers of cells positive for SV40 T Ag were observed between proximal tubules in the outer medulla, interstitial staining with $5^{\prime} \mathrm{NT}$ was weak or absent in 
this region. In addition to this regional difference in antigen expression, within a particular high power field it was often possible to discern cells which were apparently only expressing SV40 T Ag, or only 5'NT, adjacent to cells which were doubly positive (Figure 5.3D). Double staining was also observed with the fibroblast marker ER-TR7. In contrast to 5'NT, this was seen both in the cortex and outer medulla, but, as with $5^{\prime} \mathrm{NT}$, overlap was not complete with some cells expressing only SV40 T Ag or ER-TR7 lying close to doubly positive cells.

\subsubsection{Immunoelectron microscopy}

Ultrastructural examination of the mid and deep cortex showed nephron segments, capillaries and small areas of interstitial tissue between the tubules. These features were similar to those previously described in the rat (Lemley and Kriz 1991; Kaissling and Le Hir 1994). Although sub-optimally fixed because of the omission of post-fixation with osmium tetroxide, non-endothelial interstitial cells appeared to be predominantly fibroblast-like with a few rounded mononuclear cells (Figure 5.5A). The antigen-presenting cells of dendritic morphology were not separarately identified. In Kaissling and Le Hir's study these cells were identified primarily on the basis of immunolabelling. They describe them as having some ultrastructural features which were distinct from the fibroblasts; in particular the nucleus was described as usually more rounded with extended areas of dense heterochromatin. They also described these cells as having more extensive cytoplasm around the nucleus and large blunt cytoplasmic processes. Finally, the cytoplasm was less electron-dense than that of the fibroblasts. Although variation in the morphology of fibroblast-like cells was observed in the current experiments, it was not possible to distinguish two cell populations.

Fibroblast-like cells were pleomorphic in shape, presenting flattened or stellate profiles with interconnecting cytoplasmic processes between neighbouring cells (Figure 5.5A, 5.6A). In sections stained for SV40 T Ag, examination of the epithelial, endothelial and interstitial cells showed that staining was limited to the nuclei of the interstitial fibroblast-like cells (Figure 5.5B). However, not all fibroblast-like cells were positive. While the majority of these cells in the cortex of animals with a haematocrit below $20 \%$ was positively stained, many in the inner stripe of the outer medulla were negative. In all positively stained cells, the gold particles were specifically located over the electron-lucent euchromatin, with few particles over the electron-dense hetero-chromatin (Figure 5.5B, 5.6B). The specificity of the staining was confirmed by the absence of staining in the same cells in serial sections stained with rabbit anti-toxoplasma antiserum or normal rabbit serum. 


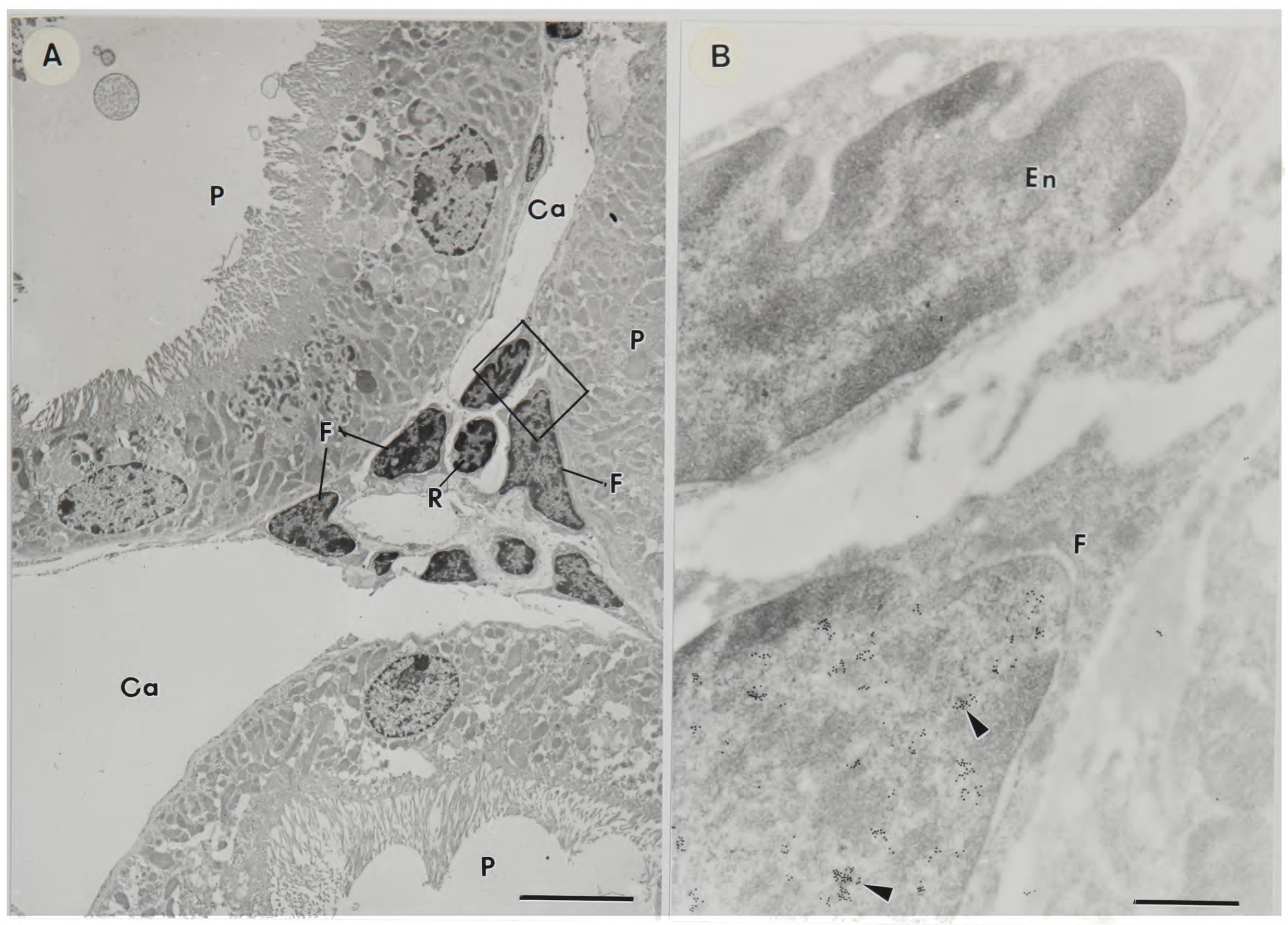

Figure 5.5 - Immunoelectron microscopy for SV40 T Ag.

$A$. Low power electron micrograph from the deep cortex showing a group of interstitial fibroblast-like cells $(F)$ and a round monocyte-like cell $(R)$ surrounded by proximal tubules $(P)$ and capillaries $(\mathrm{Ca})$. Bar is $5 \mu \mathrm{m}$.

$B$. Enlargement of the enclosed area in A showing numerous gold particles over the euchromatin of the nucleus (arrowheads) of the fibroblast-like cells $(F)$. Note the absence of label over the endothelial cell (En) nucleus. Immunostained for SV40 TAg. Bar is $0.5 \mu \mathrm{m}$. 


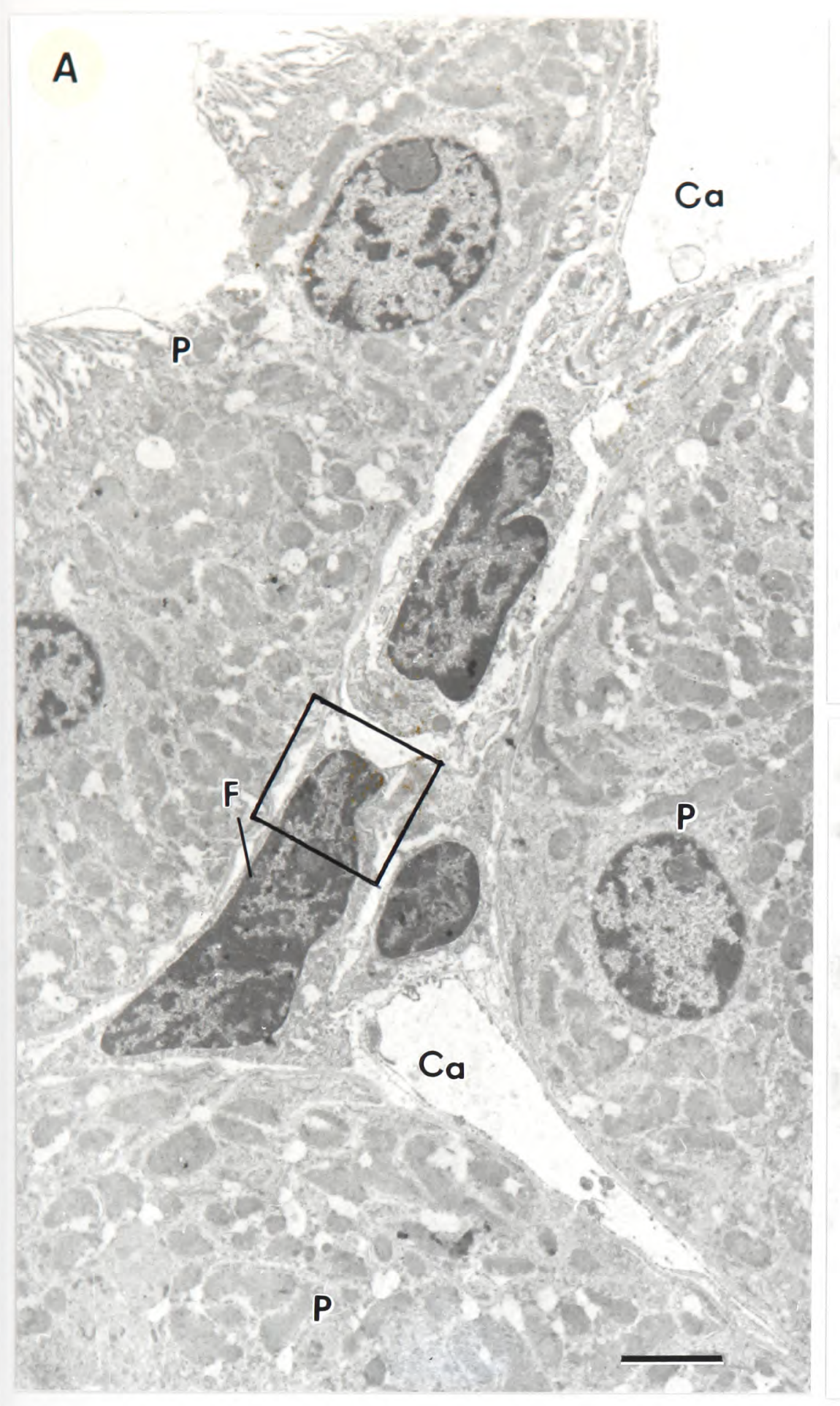

B
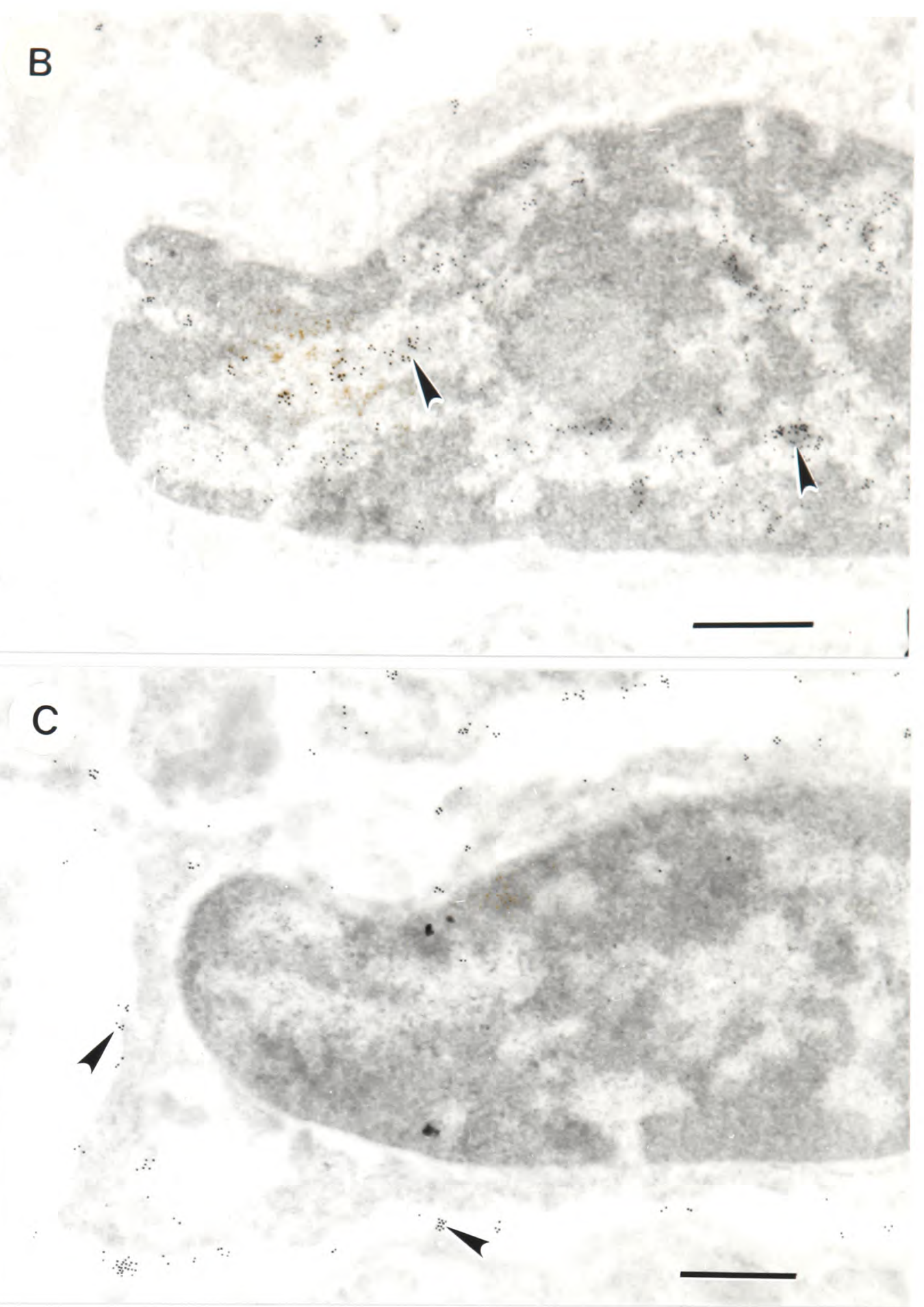

Figure 5.6 - Immunoelectron microscopy for SV40 T Ag and 5'NT.

$A$. Low power electron micrograph of three fibroblast-like cells $(F)$ between proximal tubules $(P)$ and capillaries $(\mathrm{Ca})$. Bar is $2 \mu \mathrm{m}$.

$B$. Detail of the enclosed area in A immunostained for SV4O T Ag, showing localisation of gold over the nuclear euchromatin (arrowheads). Bar is $0.5 \mu \mathrm{m}$.

C. Serial section of the same fibroblast-like cell as in B immunostained for $5^{\prime} N T$, showing localisation of gold along the plasma membrane (arrowheads). Bar is $0.5 \mu m$.

In sections labelled for $5^{\prime} \mathrm{NT}$, many of the fibroblast-like cells in the interstitium showed positive staining with the gold particles predominantly located along the plasma membrane and outlining the numerous fine cytoplasmic processes formed by these cells (Figure 5.6C). Capillary endothelial cells were negative. When serial sections stained for SV40 T Ag and 5 NT were examined, it was possible to confirm that individual cells positive for SV40 T Ag were also positive for 5'NT (Figure 5.6B and 5.6C).

Labelling for 5'NT was also observed in mesangial cells of the glomerulus and in the apical cytoplasm, but not the microvilli, of proximal tubular cells (Figure 5.7) 


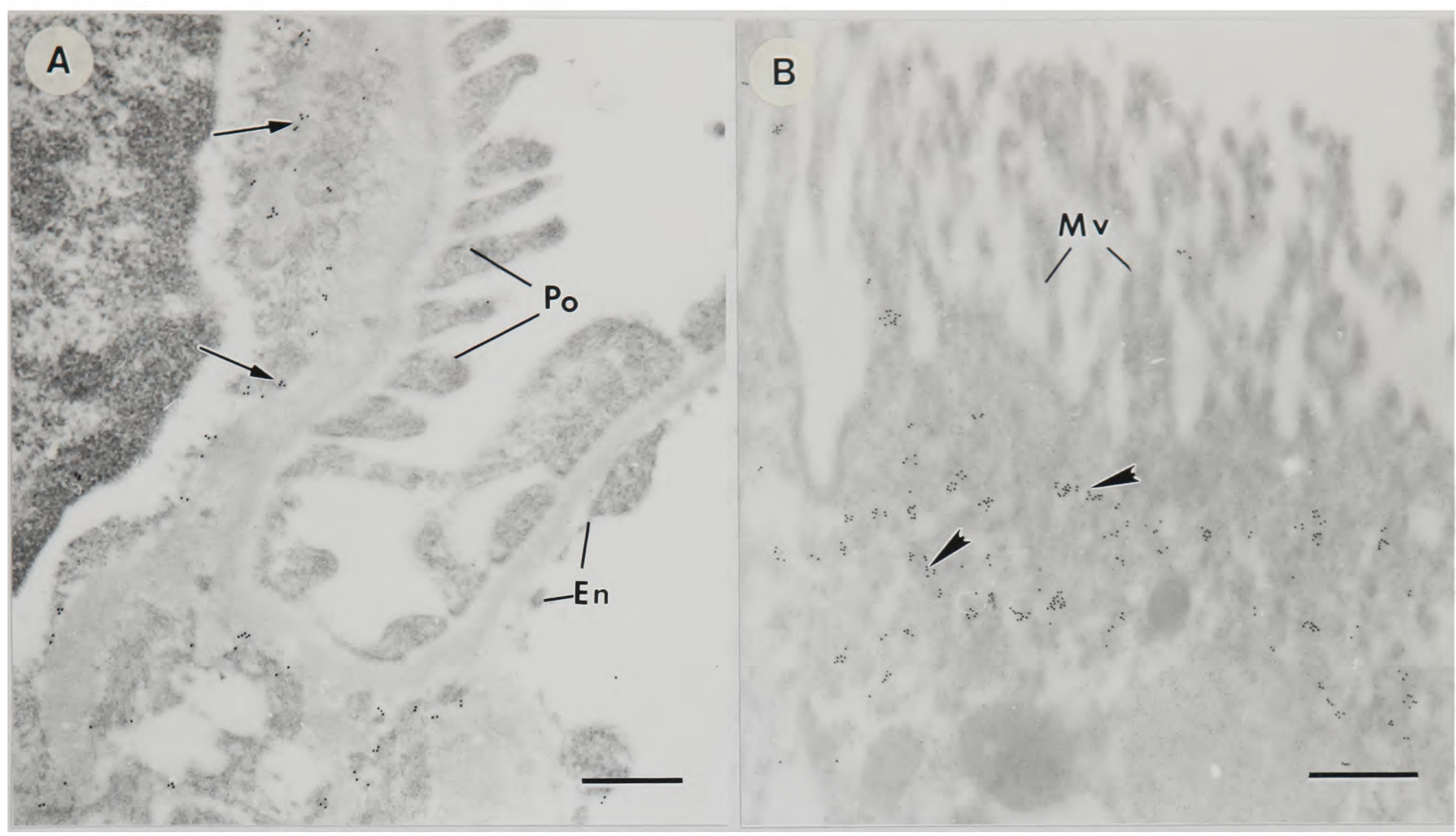

Figure 5.7 - Localisation of 5'NT by immunoelectron microscopy.

A. Electron micrograph of part of a glomerulus immunostained for 5'NT showing a positively labelled mesangial cell (arrows) while the fenestrated endothelium (En) and podocyte (Po) are unstained. Bar is $0.2 \mu \mathrm{m}$.

$B$. Section through apex of a proximal tubular cell showing labelling in the apical cytoplasm (arrowheads) and little on the microvilli (Mv). Bar is $0.5 \mu \mathrm{m}$.

\subsection{DISCUSSION}

The production of Epo-TAg mice provided an effective means for identification of the Epoproducing cells in the kidney. Nuclear staining for SV40 T Ag was readily combined with staining for cytoplasmic antigens in double immunohistochemical labelling studies and could also be detected by immunoelectron microscopy, allowing ultrastructural studies. Results from both techniques were in agreement. Interstitial cells staining with SV40 T Ag did not bear endothelial or leukocyte markers. They were distinguished easily by electron microscopy from interstitial macrophage and endothelial cells and corresponded to the cortical fibroblasts (Type 1 interstitial cells) in ultrastructural studies of kidneys from rats (Lemley and Kriz 1991). Since cells expressing SV40 T Ag did not express CD45, or Class II MHC, they are distinct from the dendritic cells of bone marrow origin.

Resident cortical fibroblasts in rodent kidneys have not been well characterised with respect to specific antigenic markers. However, renal expression of the ectoenzyme $5^{\prime} \mathrm{NT}$ has been studied in detail by immunohistochemistry and histochemistry (Dawson et al. 1989; Le Hir 
and Kaissling 1989). The enzyme is expressed strongly in the cortical interstitium as well as in proximal tubules, the intercalated cells of the distal tubule and collecting duct, and the mesangium. Although it is clear that a large number of cell types both within the kidney and elsewhere can express 5'NT, Le Hir and Kaissling (Le Hir and Kaissling 1989) have reported that this antigen is cell-type specific in the renal interstitium. Co-expression of SV40 T Ag and 5'NT was studied using double-labelling of frozen sections for light microscopy and labelling of sequential sections for electron microscopy. SV40 T Ag stained nuclei were frequently observed to be surrounded by a meshwork of 5'NT staining cytoplasm by light microscopy. A similar result was obtained using the fibroblast marker ER-TR7. Co-expression of nuclear SV40 T Ag and 5'NT on the cytoplasmic membrane of the same cell was confirmed by electron microscopy.

Thus the SV40 T Ag containing cells are the Type 1 interstitial cells, based on surface marker expression and ultrastructural characteristics. Furthermore, immunoelectron microscopy demonstrated that the cells expressing SV40 T Ag were all of fibroblast-like morphology, irrespective of whether they were in the cortex (where co-expression of 5 'NT was common), or in the outer stripe of the outer medulla (where $5^{\prime} \mathrm{NT}$ was not expressed in the interstitium). The fibroblast population therefore shows evidence of heterogeneity, both in terms of Epo production and surface molecule expression. It is interesting that $5^{\prime} \mathrm{NT}$ expression in this population is induced by anaemia; as is the case with Epo, $5^{\prime} \mathrm{NT}$ expression extends outwards from the deep to superficial cortex with more severe anaemia (Le Hir et al. 1989). The apparent overlap between 5'NT and Epo expression will therefore vary with the severity and timing of stimulation as well as with the sensitivity of the detection method. However, the absence of complete overlap indicates that it is unlikely that induction of 5 'NT expression is necessary for Epo expression or vice-versa.

Since the cellular localisation of expression depends on a marker (rather than on demonstration of Epo or Epo mRNA) it is indirect evidence for Epo production. However, several lines of argument strongly support the validity of this approach. Firstly, as described in the previous chapter the expression of Epo-TAg is closely similar to that of the native Epo gene in terms of inducibility and tissue distribution. Secondly, the cellular expression of the marker gene in the renal interstitium, the number of cells involved and the pattern of recruitment is strikingly similar to the results obtained using in situ hybridisation for Epo mRNA in the mouse (Koury et al 1989). Thirdly, in Epo-TAg ${ }^{\mathrm{H}}$ mice the marker gene is in the context of the entire native Epo locus and should behave in the same way as the native 
gene. Finally, during the course of these experiments, in situ hybridisation for Epo mRNA was succesfully combined with immunolabelling for $5^{\prime} \mathrm{NT}$ in the rat (using the same rabbit antiserum) (Bachmann et al. 1993). This study demonstrated that at least a proportion of cells containing Epo mRNA bore 5'NT, and were therefore Type 1 interstitial cells.

In situ hybridization to demonstrate that Epo mRNA and SV40 T Ag were present in the same cells was not performed in the transgenic mice. This was because the Epo-TAg transgene gave rise to transcripts which would have been indistinguishable by in situ hybridization from those arising from the endogenous Epo gene, since transcripts ran on through the SV40 polyA signals and into Epo sequence (see Chapter 8).

The identification of fibroblasts as the Epo-producing cell-type was to some extent unexpected, for the following reasons. Growth of fibroblast-like cells occurs in primary cultures of kidney cells and many established renal cell lines are classified as fibroblastoid; yet these cell cultures do not produce Epo (Jelkmann 1992). It is also notable that fibroblast proliferation is common in many forms of renal disease but that Epo deficiency is the rule. One possible explanation for this paradox is that co-operative cell interactions are required for Epo production in kidney, which are destroyed in renal disease and are not met in tissue culture. Equally, it is possible that the proliferating fibroblastoid cells may not be equivalent to the fibroblasts (Type 1 interstitial cells) in the normal kidney or that proliferation itself may reduce the facility for Epo gene expression. Experiments to address these issues are discussed in Chapter 7.

Several other incidental conclusions to the main purpose of this chapter should be noted. (1) Quantitative analysis of Epo mRNA levels in the previous chapter suggested that the level in $188.1 \mathrm{HC}$ and 176.5 was of the order of 30 fold less than that of the native Epo gene (and hence the Epo-TAg ${ }^{\mathrm{H}}$ gene at the same haematocrit). Koury has presented evidence that individual interstitial cells are recruited in an all or none fashion, and that the amount of Epo mRNA per cell is constant (Koury et al. 1989). Since SV40 T Ag can be detected in the nuclei of interstitial cells when mice of these two lines are treated with phenylhydrazine it suggests that in the heterozygous Epo- $\mathrm{TAg}^{\mathrm{H}}$ mice the levels of SV40 T Ag in the nucleus is substantially above the limit of detection. This system is likely to be a very sensitive method for detecting Epo gene expression. (2) The cellular distribution of Epo-TAg in the transgenic lines other than Epo-TAg ${ }^{\mathrm{H}}$ was also to the same cell-type as observed in Epo-TAg ${ }^{\mathrm{H}}$ mice; this adds to the evidence that the sequence in Epo-TAg is sufficient to direct cell-type specific expression in the mouse kidney. (3) SV40 T Ag was localised to the open, electron- 
lucent chromatin. This has not been commented on in the published literature, but has also been noted by another investigator (J. Butel, personal communication). (4) The immunolocalisation of 5'NT includes two new findings. Firstly, the antigen was observed in the mesangial cells in the glomerulus. This is consistent with a previous histochemical study in the mouse (Pretlow et al. 1987), and contrasts with findings in the rat (Dawson et al. 1989); thus it most probably represents a species difference. Secondly, the observation that the $5^{\prime} \mathrm{NT}$ in the tubular cells is in the apical cytoplasm rather than in the microvilli differs from a previous report in the rat kidney (Dawson et al. 1989).

Clearly it would be of great interest to correlate the expression of Epo at a cellular level with the local level of $\mathrm{pO}_{2}$. As discussed in Chapter 1, the oxygen gradients in the kidney are complex and are not well understood. However, it is intriguing that in the inner stripe, the occasional cells positive for SV40 T Ag appeared to be in the regions located further from the vascular bundles, which is consistent with the idea that (at least in this region) in the kidney local $\mathrm{pO}_{2}$ is of importance in Epo production. Cobalt is known to stimulate Epo production in humans, experimental animals and in cultured hepatoma cells. Interestingly when cobalt was administered to a heterozygous Epo-TAg ${ }^{\mathrm{H}}$ mouse the distribution of SV40 $\mathrm{T}$ Ag containing cells was similar to that observed in moderate anaemia or hypobaric hypoxia. Although this was a single experiment it does provide some evidence that the effect of cobalt may not be independent of $\mathrm{pO}_{2}$, as has been suggested (Goldberg et al. 1988). There is, in fact, direct physiological evidence for an interdependence, since hyperbaric hyperoxia can suppress Epo production in cobalt-treated rats (Necas and Neuwirt 1971).

In conclusion, Epo-TAg mice express the transgene product in an inducible fashion in a cell population which is consistent with previous in situ studies of Epo mRNA. This system has been used to identify these cells as the Type 1 interstitial cells, which are fibroblasts. 


\section{Identification of hepatic Epo-producing cells.}

\subsection{INTRODUCTION}

Although the kidney is the predominant source of Epo in adult life, the liver is an important secondary site of production. In situ hybridisation has demonstrated Epo mRNA in two cell populations in mouse (Koury et al. 1991) and rat liver (Schuster et al. 1992). One of these populations consisted of hepatocytes. However, the non-parenchymal cell population could not be identified in either species. In addition, it was not possible to assess the distribution of Epo mRNA within the liver lobule. One significant difficulty is that the amount of mRNA per cell in the liver appears to be considerably lower than in the kidney. One approach to overcome this has been to study mice over-expressing a human Epo transgene in liver (Koury et al. 1991). Human Epo mRNA was found in a pericentral distribution in hepatocytes consistent with Epo expression being related to local $\mathrm{pO}_{2}$. No expression of this transgene was observed in non-parenchymal cells.

In Chapter 5, Epo-TAg mice were used to identify the Epo-producing cells of the kidney as the Type 1 interstitial cells. Accumulation of SV40 T Ag in the nucleus was found to offer a sensitive marker for Epo gene expression. Furthermore, it proved possible to combine this method with immunolabelling and ultrastructural characterisation. In this chapter Epo$\mathrm{TAg}^{\mathrm{H}}$ mice were used to study Epo gene expression in the liver. This has resulted in the identification of the non-parenchymal Epo-producing cells as the Ito cells (also known as stellate cells, lipocytes or perisinusoidal cells). Furthermore, it was possible to identify the distribution of SV40 T Ag in the liver lobule. Since oxygen gradients in the liver are relatively well understood, this may also offer an opportunity to correlate Epo expression with regional oxygenation.

\subsection{METHODS}

The methods employed were closely similar to those used in Chapter 5 for localisation of SV40 T Ag in the kidney of Epo-TAg mice. Significant differences are noted below.

\subsubsection{Mice}

These experiments were performed on homozygous Epo-TAgH mice. These mice are anaemic, resulting in a high constitutive level of Epo-TAg expression. In order to further induce Epo-TAg gene expression, mice were either given a single dose of phenylhydrazine or exposed to an apparent altitude of 5,200m in the hypobaric chamber. Exposure to further doses of phenylhydrazine or higher altitudes was found to result in considerable mortality. 


\subsubsection{Localisation of SV40 T Ag}

The levels of SV40 T Ag observed in the liver, as judged by the intensity of staining with the polyclonal antibody, were lower than those seen in the kidney. This had two important consequences. Firstly, detection with the mAbs was not reliable, even in acetone-fixed cryostat sections from the most stimulated animals. It was possible to demonstrate an occasional cell that stained positively with PAb419 in the most anaemic mouse studied, confirming that this was not due to complete loss of the epitope. Secondly, detection in perfusion-fixed sucrose-balanced frozen material was also unreliable. Studies of SV40 T $\mathrm{Ag}$ in the liver were therefore performed with the polyclonal antiserum. The studies were restricted to either (1) cryostat sections which were acetone-fixed or (2) paraffin-embedded material (which required treatment with protease or microwaves).

\subsubsection{Double-labelling studies}

Double-labelling studies were generally performed using acetone-fixed frozen sections and the polyclonal rabbit antiserum to SV40 $\mathrm{T} \mathrm{Ag}$.

\subsubsection{Desmin}

A murine mAb directly conjugated to HRP was used to label desmin (EPOS from DAKO). In double labelling experiments both the SV40 $\mathrm{T} \mathrm{Ag}$ and desmin were visualised with peroxidase. After labelling SV40 T Ag, the sections were incubated with $0.3 \%$ hydrogen peroxide, $0.01 \mathrm{M}$ sodium azide to quench the peroxidase. In addition, the substrate used to visualise the SV40 T Ag was nickel-enhanced DAB (black), whereas for the second antigen DAB (brown) could be used as substrate. These manoeuvres, and the distinction between cytoplasmic and nuclear labelling, were successful in permitting separate identification of the two antigens.

\subsubsection{Ecto-5'-nucleotidase}

For ecto-5'-nucleotidase $\left(5^{\prime} \mathrm{NT}\right)$ it was possible to use perfusion-fixed paraffin-embedded material after protease digestion. For double-labelling with SV40 T Ag and 5'NT it was necessary to use two rabbit antisera. After the first anti-rabbit conjugate (peroxidase), normal rabbit serum was used to saturate any residual binding sites for rabbit Ig prior to application of the second antiserum. Control sections on adjacent sections on the same slide demonstrated that this manoeuvre was successful. 


\subsection{RESULTS}

\subsubsection{Epo-TAg mRNA in the liver}

In unstimulated heterozygous mice, Epo-TAg mRNA was not detected in the liver. As expected, in the homozygous Epo-TAg ${ }^{\mathrm{H}}$ mice, chronic anaemia results in a high level of expression of the Epo-TAg gene in the kidney and a low, but detectable level in the liver. Mice were rendered more anaemic using phenylhydrazine; this resulted in increased levels of Epo-TAg mRNA in the liver of both heterozygous and homozygous Epo-TAg ${ }^{\mathrm{H}}$ mice (Figure 6.1). Interestingly, in the liver the expression of Epo-TAg mRNA in heterozygotes was substantially lower than that of the native Epo gene in all animals studied.

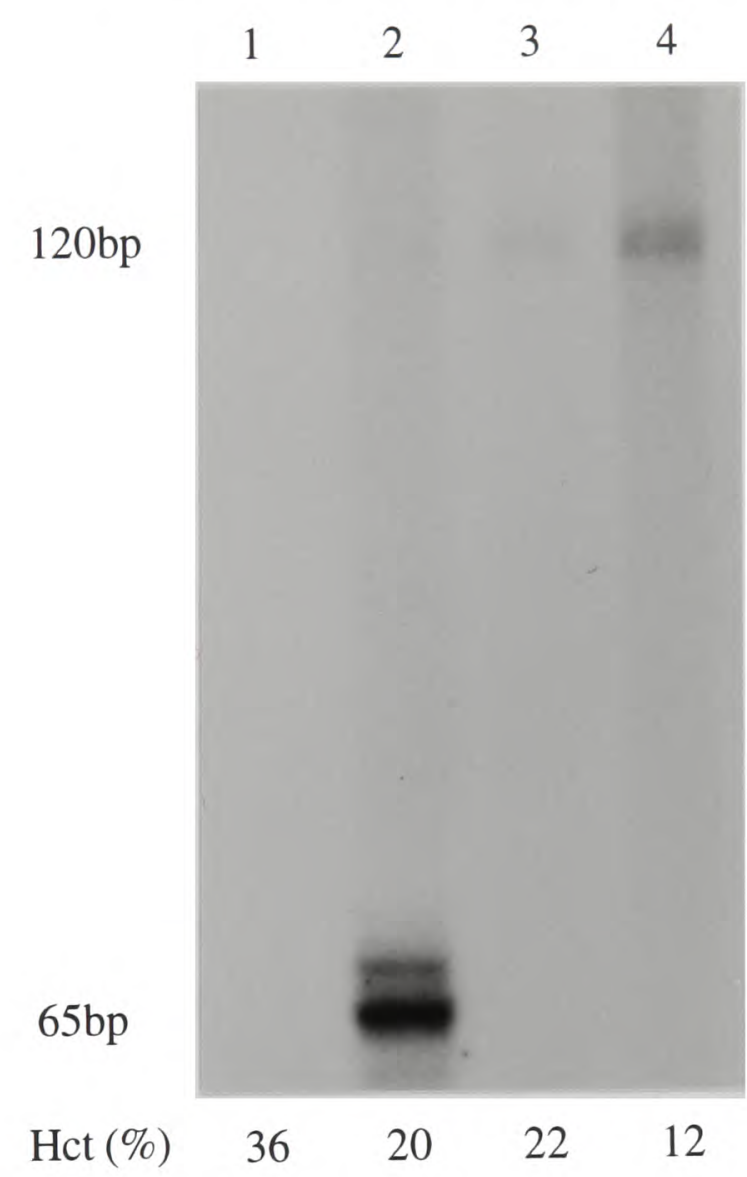

Figure 6.1 - RNase protection assay showing anaemia-inducible expression of the Epo-TAg gene in the liver of heterozygous and homozygous Epo-TAg ${ }^{H}$ mice. $500 \mu \mathrm{g}$ RNA was analysed from each liver. The Epo-TAg $m R N A$ produces a protected species $120 \mathrm{bp}$ in length. Lanes 1 and 2 contain RNA from heterozygous Epo-TAgH mice, lanes 3 and 4 contain RNA from homozygotes. In lanes 2 and 4 the mice were given phenylhydrazine prior to sacrifice. Below each lane is the haematocrit at the time of sacrifice. A protected fragment of $65 \mathrm{bp}$ arising from the native Epo gene is also present in lane 2 and is clearly more intense than the band from the Epo-TAg gene.

\subsubsection{Localisation of SV40 T Ag in the liver}

Immunohistochemical studies of SV40 T Ag expression were performed on both heterozygous and homozygous Epo-TAg ${ }^{\mathrm{H}}$ mice; adult animals, aged one to six months, were used. When compared with non-transgenic controls no abnormality of hepatic morphology was discerned, either in heterozygotes or homozygotes. However, following treatment with phenylhydrazine, occasional centrilobular cell damage was sometimes observed both in transgenic and non-transgenic mice. 
Initial studies of the cellular localisation and distribution of hepatic SV40 T Ag expression were performed on cryostat sections using the polyclonal rabbit antiserum. In unstimulated heterozygote Epo-TAg ${ }^{\mathrm{H}}$ mice no staining for SV40 T Ag was detected, consistent with the lack of detectable Epo-TAg mRNA in the liver. In the chronically anaemic homozygous Epo-TAg ${ }^{\mathrm{H}}$ mice, a very few cells were positive for SV40 T Ag. These were located away from identifiable portal triads, near the centre of the lobule. A similar number and distribution of positive cells was observed in heterozygous Epo-TAg ${ }^{\mathrm{H}}$ mice subjected to acute anaemic stimulation with phenylhydrazine. In contrast, severe stimulation, achieved by exposing homozygous Epo-TAg ${ }^{\mathrm{H}}$ mice to hypobaric hypoxia or phenylhydrazine-induced haemolysis resulted in a substantially increased (approximately twenty-fold) number of SV40 T Ag positive cells, which were located at increased distances from the central vein, but a clear pericentral distribution was still observed. The pattern of induced expression of SV40 T Ag was pericentral irrespective of whether the hypoxic stimulus was acute anaemia, chronic anaemia or hypobaric hypoxia. No staining was ever observed in sections from nontransgenic control mice (with or without phenylhdrazine treatment), or when normal rabbit serum was substituted for the polyclonal antiserum on sections from the transgenic mice.

Perfusion-fixed, paraffin-embedded sections permitted the combination of localising SV40 $\mathrm{T}$ Ag with improved morphological preservation. Two different populations of cells were found to contain SV40 T Ag. One was characterised by relatively irregular-shaped nuclei with little identifiable cytoplasm (Figure 6.2A). The other, which was less frequent, consisted of hepatocytes, with large rounded nuclei and plentiful cytoplasm (Figure 6.2B).

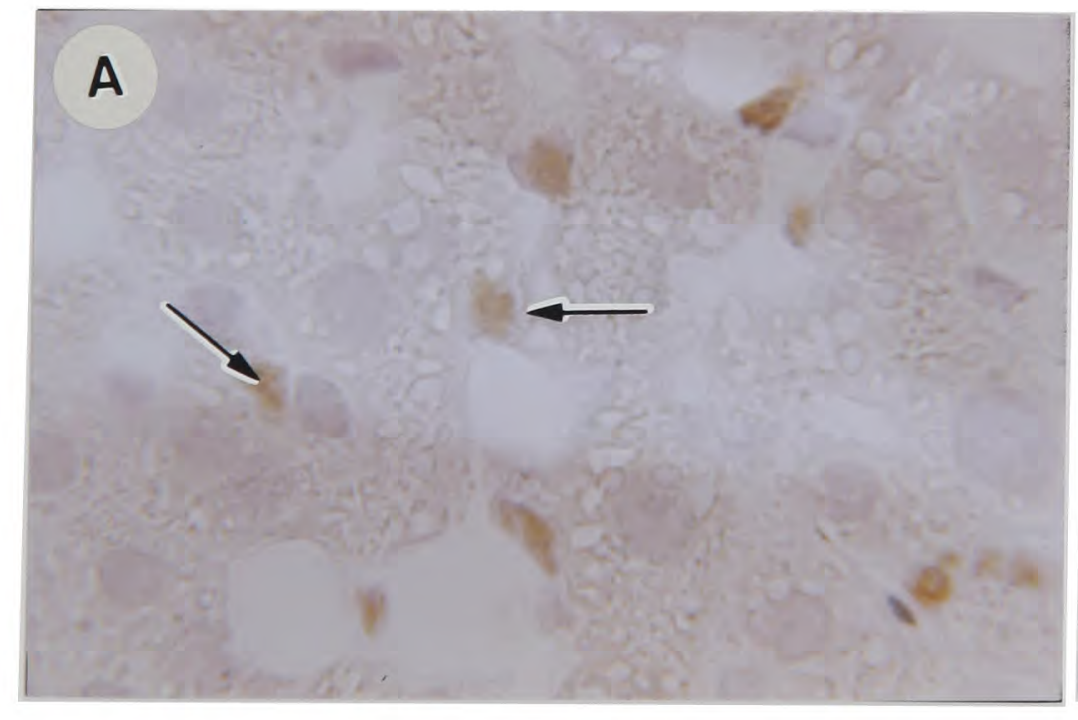

Figure 6.2 - Immunostained sections from livers of phenylhydrazine-treated homozygous Epo$T A g^{H}$ mice. In each case the region shown is from the pericentral region of a liver lobule.

A. Perfusion-fixed liver labelled for SV4O T Ag. Positive nuclei, stained brown (arrows) are seen in cells lying adjacent to hepatocytes and the sinusoidal lumen (S). Magnification $x 600$. $B$. Perfusion-fixed liver labelled for SV4O TAg. Positive staining (brown) is demonstrated in a hepatocyte nucleus, which lies adjacent to a central vein (CV). Magnification $x 600$. 


\subsubsection{Double immunohistochemical labelling studies}

In addition to hepatocytes, the liver contains several non-parenchymal cell types. These include the macrophage/monocyte-related Kupffer cells, the lymphocyte-related pit cells, the sinusoidal endothelial cells and the perisinusoidal Ito cells (Bouwens et al. 1992). As in the studies on the kidney, immunohistochemical detection was readily combined with detection of cytoplasmic and cell surface markers in double labelling studies (Table 6.1).

\begin{tabular}{|l|c|c|l|}
\hline \multicolumn{1}{|c|}{ Antigen } & Clone & Description & \multicolumn{1}{c|}{ Labelling in murine liver } \\
\hline CD45 & M1/9.3.4.HL2 & rat mAb & Kupffer cells, pit cells \\
\hline Fc receptor & $2.4 \mathrm{G} 2$ & rat mAb & $\begin{array}{l}\text { Kupffer cells, sinusoidal } \\
\text { endothelium }\end{array}$ \\
\hline CD31 & MEC13.3 & rat mAb & Endothelial cells \\
\hline Macrophage marker & F4/80 & rat mAb & Kupffer cells \\
\hline Ecto-5'-nucleotidase & $\mathrm{n} / \mathrm{a}$ & rabbit a/s & Ito cells, biliary canaliculi \\
\hline Desmin & $\mathrm{D} 33$ & EPOS-mAb & Ito cells \\
\hline
\end{tabular}

Table 6.1 - Antibodies used to characterise non-parenchymal liver cell populations. a/s = antiserum.

Antibodies to three such markers were used to label Kupffer cells; rat mAb F4/80 to a surface antigen of mouse macrophages (Austyn and Gordon 1981), rat mAb M1/9.3.4.HL2 against leucocyte common antigen (CD45), and the rat mAb 2.4G2 which binds to low affinity Fc receptors expressed on Kupffer cells and endothelial cells (Unkeless 1979; Lee et al. 1986). These antibodies all labelled non-hepatocyte populations when applied in isolation, or in combination with SV40 T Ag staining. However, there was no overlap with the population of cells expressing SV40 T Ag. Leucocyte common antigen (CD45) is expressed not only by Kupffer cells but also by the lymphocyte-related pit cells, indicating that both these cell populations are distinct from the cells expressing SV40 T Ag (Figure 6.3A). Endothelial cells in the liver express platelet endothelial cell adhesion molecule (PECAM/CD31), which was labelled using rat $\mathrm{mAb}$ MEC13.3. Figure 6.3B shows a nucleus containing SV40 T Ag closely apposed to the nucleus and cell surface of an endothelial cell. In no instance was double labelling for SV40 T Ag and CD31 observed. This result is in keeping with the studies using $2.4 \mathrm{G} 2$, indicating that the cells expressing SV40 T Ag are not endothelial. 

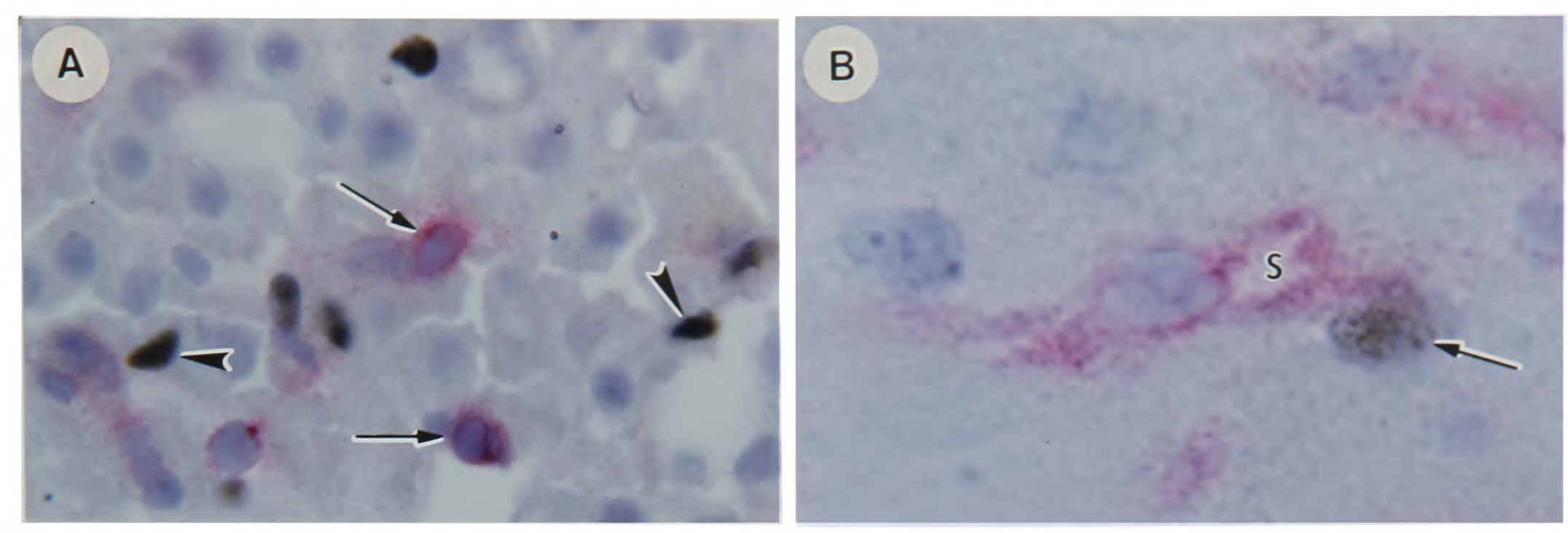

Figure 6.3 - Double labelling for SV4O T Ag and surface markers.

A. Frozen section labelled for SV4O T Ag (black) and leukocyte common antigen (CD45, red). Several non-parenchymal cells are seen to bear CD45 (arrows). A different population shows nuclear staining for SV4O TAg (arrowheads). No double-labelling is observed. Magnification $x 450$.

B. Frozen section labelled for SV4O T Ag (black) and CD31 (red). A single nucleus containing SV4O T Ag (arrow) lies immediately adjacent to a small sinusoid (S) lined by an endothelial cell, the nucleus of which is seen not to contain SV4O T Ag. Magnification $x 900$.

Ito cells possess long processes extending between the hepatocytes and express the intermediate filament protein, desmin, which provides a specific marker for these cells in rodent liver (Yokoi et al. 1984). Storage of Vitamin A in lipid droplets is observed in most Ito cells but is most marked in the periportal regions (Bouwens et al. 1992). Using a directly conjugated mouse $\mathrm{mAb}$ to desmin, characteristic staining of fine processes extending throughout the hepatic lobule was observed. When the anti-desmin $\mathrm{mAb}$ and antiserum to SV40 T Ag were combined, double staining was observed in individual cells (Figure 6.4A, $6.4 \mathrm{~B}$ ). The majority (possibly all) of the non-parenchymal pericentral cell population expressing SV40 T Ag also stained for desmin. However, desmin-positive cells lying in the periportal region did not express SV40 T Ag even in the most stimulated animals. 


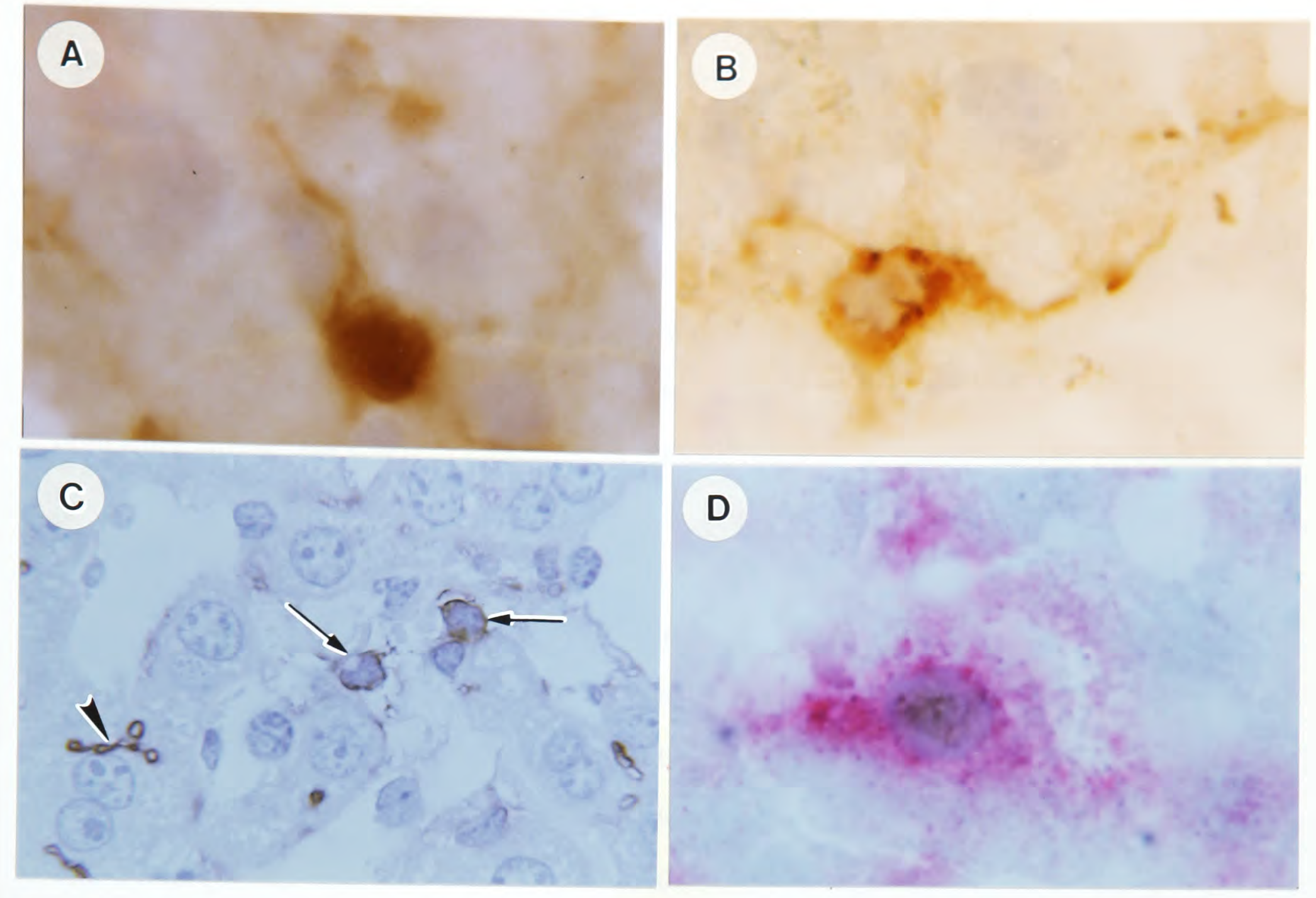

Figure 6.4 - Double labelling for SV4O TAg and surface or cytoplasmic markers borne by Ito cells.

A. Frozen section labelled for SV4O TAg (brown, nuclear) and desmin (brown, cytoplasmic). An Ito cell with desmin positive processes is stained positively for SV4O TAg. Magnification $x 1200$.

B. Adjacent frozen section stained as in A, but with normal rabbit serum substituted for the antiserum to SV4O TAg. The fine desmin-positive processes of an Ito cell are shown. No staining is observed over the nucleus. Magnification $x 1200$.

C. Perfusion-fixed, paraffin-embedded section labelled for 5'NT (brown). Two nonparenchymal cells (arrows) are seen to bear 5'NT, identifying them as Ito cells. Staining of the biliary canaliculi of hepatocytes is also observed (arrowhead). Magnification $x 600$.

D. Frozen section labelled for SV40 T Ag (brown, nuclear) and 5'NT (red). An Ito cell bearing 5'NT is seen to contain SV40 T Ag. Magnification x 1200.

Ecto-5'-nucleotidase ( $\left.5^{\prime} \mathrm{NT}\right)$ is located on the biliary canaliculi of hepatocytes and on Ito cells in rodent liver (Schmid et al. manuscript submitted). Using the same polyclonal rabbit antiserum to localise $5^{\prime} \mathrm{NT}$ in the anaemic Epo-TAg ${ }^{H}$ mice, labelling was seen in a pericentral distribution in the liver. In both perfusion-fixed material and frozen sections it was possible to identify staining of both biliary canaliculi and of non-parenchymal cells (Figure 6.4C). When visualisation of 5'NT was combined with labelling of SV40 T Ag, SV40 T Ag was observed in the nuclei of cells expressing a high level of 5'NT (Figure 6.4D). Although a high proportion (possibly all) of the non-parenchymal SV40 T Ag positive cells were positive for $5^{\prime} \mathrm{NT}$, many 5 'NT positive cells in the periportal regions did not contain SV40 T Ag. 


\subsubsection{Immunoelectron microscopy}

Initially the ultrastructural features of the various cell types within the liver were examined in optimally fixed tissue. The appearance was similar to that previously described for rodent liver. The endothelial cells formed the sinusoids, with thin cytoplasmic processes interrupted by gaps and fenestrations. The Kupffer cells were normally located within the sinusoids and characterised by a variable number of large heterophagosomes containing debris. Rare lymphocyte-like cells were seen within the sinusoids which probably represented the pit cells. The space of Disse, between the sinusoidal endothelium and the hepatocytes, contained numerous small cytoplasmic processes from the hepatocytes and a number of Ito cells. These cells were normally in close apposition to the hepatocytes and were characterised by a variable number of large lipid droplets, which in many cases caused displacement or indentation of the nucleus. In the osmium-fixed material the lipid droplets were of a homogenous, slightly electron-dense appearance (Figure 6.5A). The only other cells with lipid droplets were a small number of hepatocytes, which were identifiable by their other organelles. Material for immunoelectron microscopy was not fixed with osmium tetroxide and consequently was less well preserved ultrastructurally. However, it was possible to distinguish the cell types as described above. The Ito cells were identified within the space of Disse on the basis of the large cytoplasmic fat droplets which appeared as empty vacuoles, due to lipid extraction in the absence of osmium tetroxide (Figure 6.5B). 


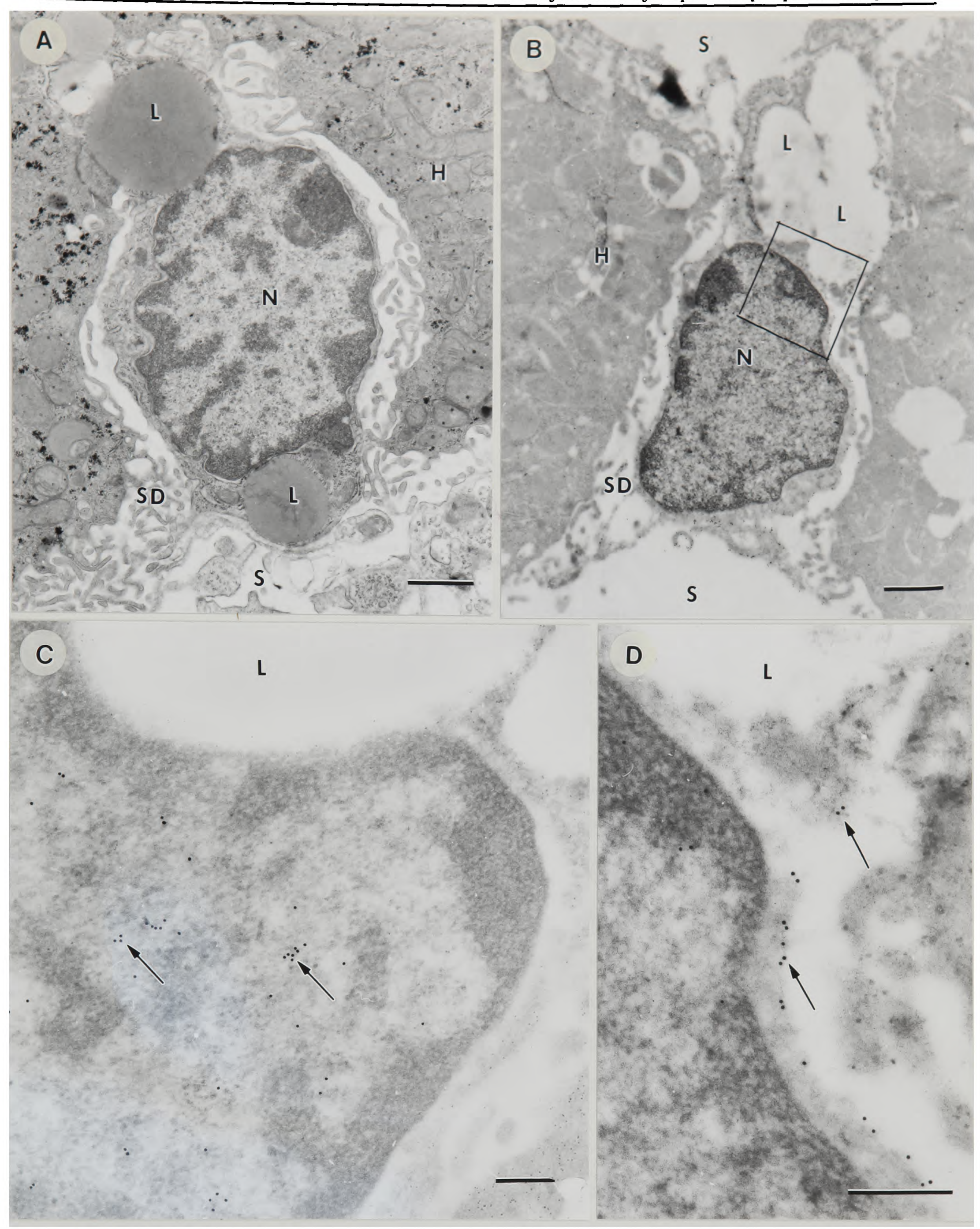

Figure 6.5 - A. Conventional transmission electron micrograph showing an Ito cell characterised by the presence of lipid droplets ( $L$ ) located in the space of Disse $(S D)$ between hepatocytes $(H)$ and a sinusoid $(S) . N=$ nucleus. Bar is $1 \mu \mathrm{m}$.

B. Electron micrograph through an Ito cell in a sample processed for immunocytochemistry showing the cell lying in the space of Disse subtended by hepatocytes and sinusoids. Note the reduced quality of fixation and the extraction of the lipid droplets. Bar is $1 \mu \mathrm{m}$.

C. Detail of part of an Ito cell immunostained for SV4O T Ag showing labelling with gold particles (arrows) over the euchromatin. Bar is $0.2 \mu \mathrm{m}$.

$D$. Enlargement of the enclosed area in B immunostained for 5 'NT showing the gold particles located along the plasmalemma of the Ito cell (arrows). Bar is $0.2 \mu \mathrm{m}$. 
In sections stained for SV40 T Ag, staining was observed in the nuclei of Ito cells (Figure $6.5 \mathrm{C})$. However, not all Ito cells were labelled with gold particles. In positively stained cells, more gold particles were located over the electron-lucent euchromatin than over the electron-dense heterochromatin. Although the number of gold particles was relatively low it was markedly greater than that seen over the nuclei of other cell types and in negative controls, thus confirming the specificity of the staining. In sections stained for 5 NT, staining was observed on the cell membrane of Ito cells, and on their fine processes in the space of Disse (Figure 6.5D).

\subsection{DISCUSSION}

In these experiments, expression of the Epo-TAg transgene has been used to identify liver cells with the potential for Epo gene expression. The justification for this approach in the kidney was discussed in Chapter 5. As in the kidney the correlation with previous in situ hybridisation studies provides supportive evidence that this is a valid approach. Thus the non-hepatocytic liver cell population is in keeping with the in situ hybridisation studies previously reported by others (Koury et al. 1991; Schuster et al. 1992). Immune detection of SV40 $\mathrm{T} \mathrm{Ag}$ provided the potential for further characterization of these cells.

Previous attempts to define this cell population have not been successful, although it has been suggested that the Kupffer cells can produce Epo (Paul et al. 1984). The cell population defined in the current experiments as expressing SV40 T Ag did not express leukocyte, macrophage or endothelial markers and therefore contains neither Kupffer nor endothelial cells. Immunohistochemical and ultrastructural characteristics demonstrate that the cells expressing SV40 T Ag are the Ito cells. The Ito cells have also been described as fat-storing cells, lipocytes, stellate cells or perisinusoidal cells (Ramadori 1991). They are not a rare cell type in the liver, and it is estimated that there is approximately one Ito cell for every two hepatocytes.

SV40 T Ag expression in Ito cells was undetectable in unstimulated heterozygous animals, at the limit of detection in the chronic anaemic homozygous animals, but could be readily detected following further stimulation with hypobaric hypoxia or phenylhydrazine induced haemolysis. In these severely stimulated animals a clear lobular distribution of positive cells was observed in the pericentral regions. Such a distribution is in keeping with known oxygen gradients within the liver (Jungermann and Katz 1989) and indicates that the cells 
are most probably responding directly to a low partial pressure of oxygen. Interestingly a similar distribution of Epo mRNA was observed by Koury among hepatocytes of transgenic animals which overexpressed a human Epo transgene in liver (Koury et al. 1991).

The intensity of staining for SV40 T Ag observed in individual nuclei in the liver, both in hepatocytes and non-parenchymal cells, was lower than that seen in kidney from the same animal. Within the limits of such a comparison, this is consistent with in situ hybridisation studies of Epo mRNA in which slides of liver required longer exposure times than those of kidney (Koury et al. 1991; Schuster et al. 1992), and suggests that the expression of Epo per cell in the liver is lower than in the kidney.

Although Epo-TAg expression was also observed in hepatocytes, this accounted for a relatively low proportion of the cells positive for SV40 T Ag (less than 10\%). The estimated contributions of the hepatocytes and non-parenchymal cells in other studies of liver Epo expression has varied rather widely. Using cell separation techniques, Lacombe et al. reported greater Epo mRNA content in non-parenchymal liver cells than in hepatocytes prepared from anaemic mice (Lacombe et al. 1991). Kurtz et al describe the Epo mRNA containing cells in carbon monoxide stimulated rats as lying between hepatocytes; hepatocytes were not positive for Epo mRNA by in situ hybridisation in this study (Kurtz et al. 1989). However, in a substantially more detailed study, Koury et al., also using in situ hybridisation on anaemic mouse liver found that many more hepatocytes expressed Epo mRNA than non-parenchymal cells (Koury et al. 1991). A similar result was obtained by Schuster et al. in anaemichypoxic rat liver (Schuster et al. 1992).

It is certainly possible that the method used to stimulate hepatic Epo production alters the relative contribution of these two cell populations. In the current experiments the stimulus employed was hypoxia or haemolysis on the background of a chronic anaemia, and this may have resulted in differential adaptive changes in Epo expression in hepatocytes and Ito cells. Alternatively, it is possible that the insertion of the SV40 T Ag coding sequence in Epo-TAg influenced the relative level of expression of the gene in these cells, in spite of the preservation of all Epo sequence. This could, for instance, be due to the distancing of the $3^{\prime}$ enhancer region from the Epo promoter. Some support for this possibility was provided by the RNase protection studies of EpoTAg mRNA and native Epo mRNA in heterozygous animals. The level of Epo-TAg mRNA in the liver was at least four-fold lower than that of native Epo mRNA. In contrast, in the kidney the levels of Epo-TAg and native Epo mRNA were very similar (mean ratio 1.0 , range 0.7 to 1.5 ). It is therefore possible that in the 
kidney and Ito cells the Epo-TAg gene is expressed at a quantitatively similar level to the native gene, but that in hepatocytes the insertion of SV40 sequence has led to reduced expression. This is further supported by the difference between expression of the Epo-M and Epo-TAg transgenes, where the insertion of the SV40 sequence appeared to abrogate the over-expression of Epo-M in the liver (see Chapter 4). In addition, transient transfection studies using a $10 \mathrm{~kb}$ construct containing the Epo-TAg gene (with $0.4 \mathrm{~kb} 5$ flanking sequence and $3.5 \mathrm{~kb}$ of $3^{\prime}$ flanking sequence) did not produce detectable expression in HepG2 cells, in contrast to the same construct without the SV40 sequence (P. J. Ratcliffe, unpublished data). A final possible explanation for predominant Ito cell expression should be considered. The expression of SV40 T Ag in Ito cells may have led to expansion of this population, although this was not evident when liver histology was compared with that of non-transgenic mice.

Epo mRNA has been identified in a non-hepatocyte population separated by metrizamide gradient centrifugation (Schuster et al. 1992). The authors did not comment on whether their use of this technique would enable purification of Ito cells, but similar methods have been applied to separate Ito cells on the basis of the altered buoyant density arising from fat storage (Ramadori 1991). Such methodology might represent a way of obtaining Epoproducing Ito cells in tissue culture, but there are potential problems. In particular, although some fat-storing cells did express SV40 T Ag, fat storage is not universal in the Ito cell population, and the most heavily fat-laden cells are in the periportal zones where SV40 T $\mathrm{Ag}$ and presumably Epo gene expression is not observed in vivo (Bouwens et al. 1992).

In response to vitamin $\mathrm{A}$ administration Ito cells are known to accumulate lipid. Prior administration of vitamin $A$ has therefore been used to facilitate the separation of Ito cells from the liver (Ramadori 1991). Furthermore, in response to vitamin A lipid droplets are seen in Ito cells in the periportal region of the liver lobule. This might offer an important advantage in purifying Epo-expressing cells from the liver. However, vitamin A accumulation is also known to alter Ito cell behaviour. A preliminary experiment was therefore performed to determine the effects of vitamin A administration on Epo gene expression. This suggested that prior administration of 30,000 i.u. of vitamin A ten days before hypoxic exposure did not alter the hepatic induction of Epo-TAg or Epo mRNA, and did load Ito cells throughout the lobule. Preliminary experiments indicate that a reasonably pure $(>70 \%$ on electron microscopy) preparation of Ito cells prepared from the liver of an anaemic rat contain a substantial amount of Epo mRNA (Figure 6.7). 


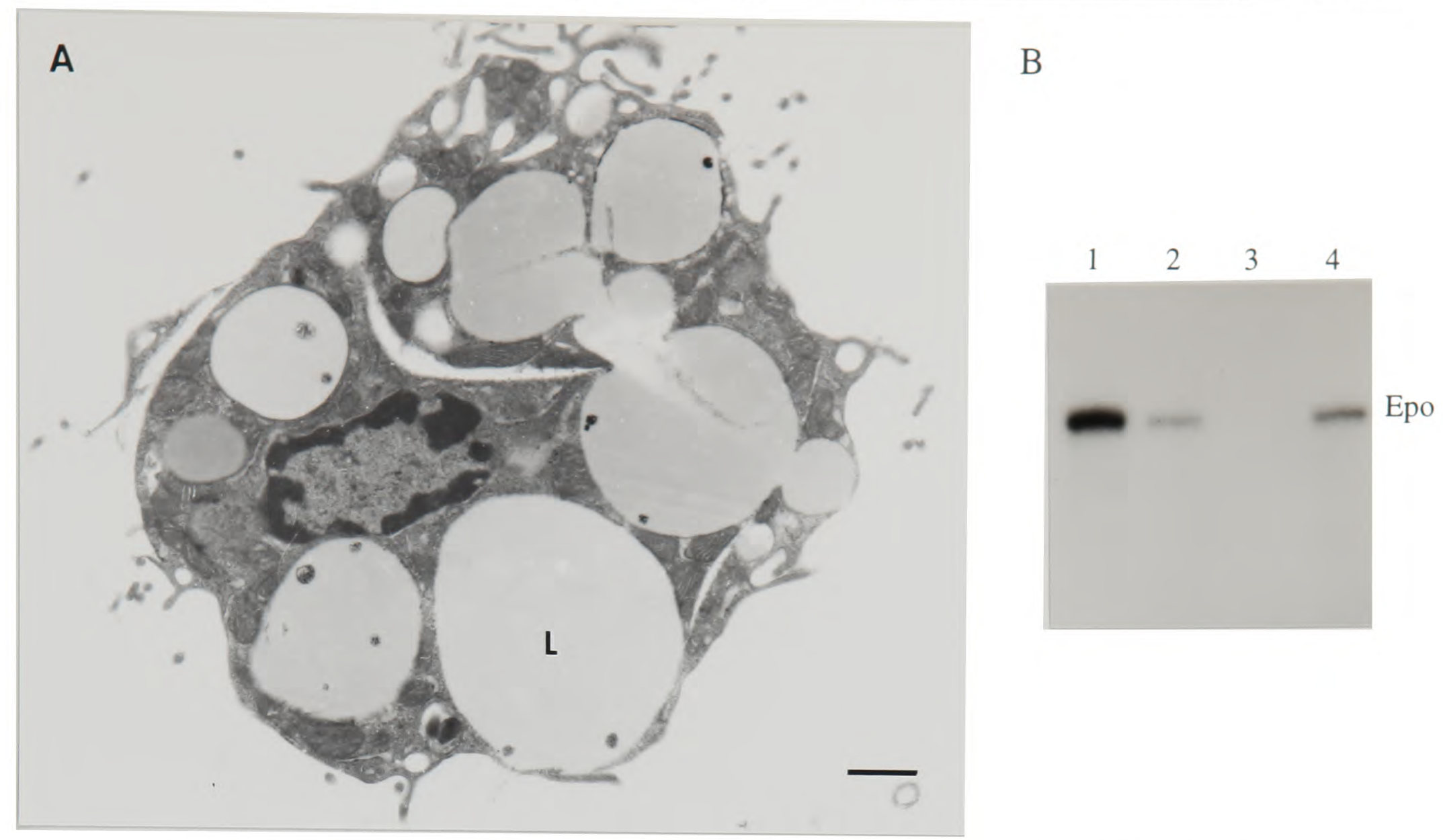

Figure 6.7 - A. Electron micrograph showing an Ito cell with characteristic lipid droplets $(L)$, isolated by perfusion-digestion of the liver of an anaemic rat, density gradient centrifugation and elutriation. This fraction contained $>70 \%$ lipocytes, with some Kupffer cells and a few non-viable hepatocytes. Bar is $1 \mu \mathrm{m}$.

B. RNase protection assay showing Epo mRNA in the lipocyte fraction (lane $1 ; 280 \mu \mathrm{g}$ total $R N A$ ) and to a lesser extent in the Kupffer cell fraction (lane 2; $140 \mu \mathrm{g}$ total RNA). The latter is probably due to hepatocyte contamination (which was substantially more marked in this fraction). Lane 3 contains no RNA, lane 4 contains unstimulated rat kidney RNA (250 $\mu \mathrm{g})$.

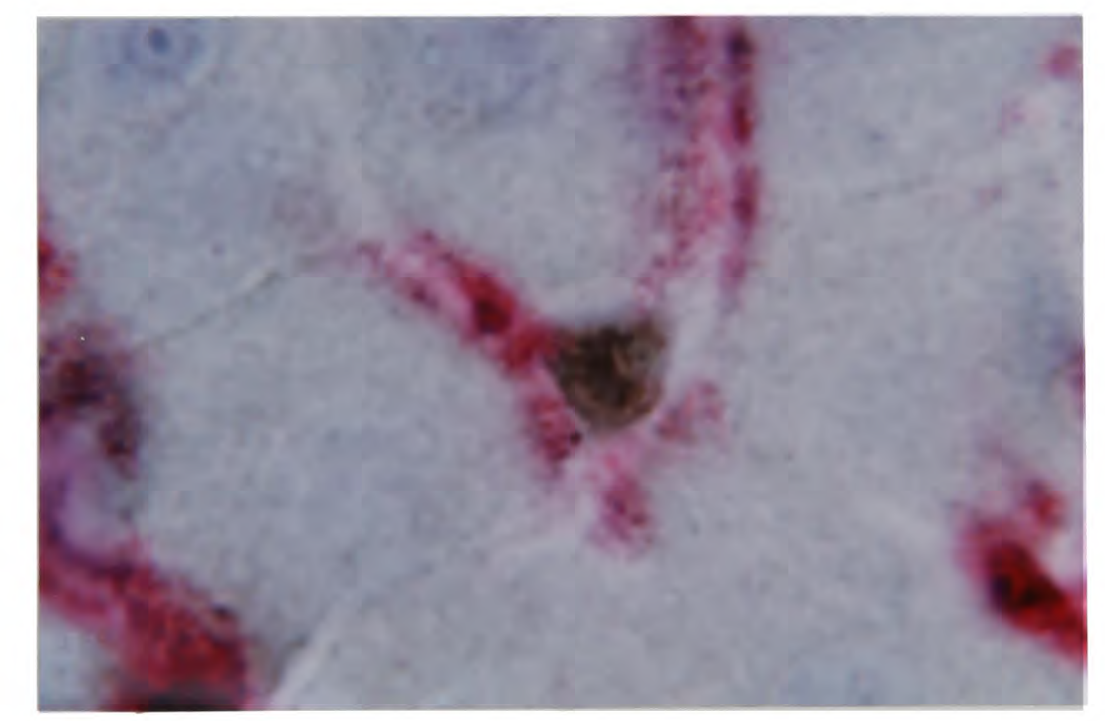

Figure 6.8 - Double labelling of a non-parenchymal cell for ER-TR7 (red) and SV4O T Ag (brown). The processes of this cell extend between the hepatocytes, outlining the sinusoids. Magnification $\times 600$.

There are several phenotypic similarities between the fibroblastoid Epo-producing Type 1 interstitial cells of the renal cortex and outer medulla, and the Ito cells of the liver. Both are closely apposed to parenchymal and endothelial cells and possess extensive fine processes (Lemley and Kriz 1991; Ramadori 1991). In both organs they are the major connective tissue producing cells, and they proliferate in response to injury (Ramadori 1991; Nagle et al. 1973). Fibroblasts from foetal mouse kidney (Woolf et al. 1993) and Ito cells (Schirmacher 
et al. 1992), secrete hepatocyte growth factor, which appears to influence the growth and differentiation of tubular cells (Montesano et al. 1991) as well as hepatocytes. As with the renal cell population, Ito cells express 5 NT. As discussed in Chapter 5 , in the renal interstitium expression of this surface enzyme is specific for Type 1 interstitial cells, and expression is increased in response to anaemia. It is of interest that 5 'NT expression in the liver shows zonation in the lobule, with higher expression in the less well oxygenated pericentral region. During the course of this study Kaissling and colleagues demonstrated that $5^{\prime} \mathrm{NT}$ expression on Ito cells is increased in response to anaemia (Schmid et al. manuscript submitted). A subset of the renal Type 1 interstitial cells express the fibroblast-marker ERTR7 (Chapter 5). Figure 6.8 shows double-labelling for SV40 T Ag and this marker in the liver of stimulated homozygous Epo-TAg ${ }^{\mathrm{H}}$ mice. In the normal mouse kidney interstitial cells label with the mAb to desmin, and in anaemic Epo-TAg ${ }^{\mathrm{H}}$ mice double-labelling for SV40 T antigen and desmin shows considerable overlap between these populations (data not shown). In response to injury the interstitial cells in the kidney become more myofibroblastoid with smooth muscle actin expression (Nagle et al. 1973) and increased desmin expression (see Chapter 7). Similar phenotypic changes have been reported in Ito cells after liver injury, and after explantation of Ito cells in tissue culture (Ramadori 1991). It thus appears possible that the failure to obtain Epo producing cells from these populations in tissue culture relates to such phenotypic changes; this forms the subject of experiments described in Chapter 7. 


\section{Biology of renal Epo-producing cells}

\subsection{INTRODUCTION}

This chapter is concerned with the Epo-producing cells in the kidney. As discussed in Chapter 1 knowledge concerning the characterisation and behaviour of these cells has been limited, and hitherto their identity has been unknown. The experiments described in Chapter 4 and the in situ hybridisation experiments of Bachmann et al. (Bachmann et al. 1993) have identified the renal Epo-producing cells as the Type 1 interstitial cells. Clearly there is much to learn about the biology of these cells. For instance, a striking feature of kidney disease is the failure of the kidneys to maintain Epo levels sufficient for a normal haematocrit. As discussed in Chapter 1, cell culture lines capable of producing significant amounts of Epo in a regulated fashion have not been isolated from the kidney, despite many attempts. The reason for this is not understood. These observations raise important questions about the biology of these cells and the circumstances which permit them to express the Epo gene.

Both in tissue culture and in diseased kidney there appears to be a lack of Epo production. In principle there could be several different causes of this. For instance, the cells expressing the gene could be absent. Alternatively, the correct stimulus to Epo production in the normal kidney (or an essential component of it) might be lacking. Finally, the cells might be present, but have undergone a phenotypic alteration preventing them from expressing the gene.

The oxygen sensing mechanism underlying hypoxic regulation of the Epo gene can be studied in a wide range of cell types, as shown in Chapter 3. However, the interaction of this mechanism with other aspects of Epo gene regulation requires a model system in which the Epo gene is expressed. Although hepatoma cells have provided an important and useful model for Epo production, they are clearly of limited value in addressing these issues. They do provide two important pieces of information; that detection of hypoxia and Epo production can occur in the same cell type and that regulated Epo production can occur in a tissue culture cell line. However, these epithelial tumour cells are clearly dissimilar from the fibroblastoid interstitial cells of the kidney. In addition, the transgenic data of Semenza and that described in Chapters 4 and 6 of this thesis demonstrate that there are differences in the control of Epo gene expression in the hepatocyte (for which the hepatoma cell may be a good model) and in the fibroblastoid cells of the kidney and Ito cells of the liver. 
Therefore it is an important aim to obtain the Epo-producing cells of the renal interstitium in tissue culture. Three aspects of the experiments already described in this thesis can potentially be exploited. Firstly, the cell type has been identified as a population which can be distinguished on the basis of morphology and antigenic markers, rather than solely on the basis of Epo production. Secondly, in Epo-TAg mice accumulation of SV40 T Ag in the nucleus serves as an indirect marker of Epo gene expression. Thirdly, the expression of Epo-TAg in these cells results in the presence of a transforming oncogene; a strategy that has led to selective expansion and immortalisation of other differentiated cells. This technique has been used succesfully in the islet cells of the pancreas to expand the beta cell population, with subsequent tumour formation (Hanahan 1985). Similarly, SV40 T Ag under the control of a thyroglobulin promoter resulted in development of thyroid tumours, and the isolation of cell lines (Ledent et al. 1991).

Section 7.3.1 is concerned with the effect of expressing SV40 T Ag in Epo-producing cells in transgenic mice. These mice developed polycythaemia and tumours. However, the polycythaemia was found to be due to production of active Epo by the Epo-TAg transgene outside the kidney (rather than to an expansion of renal Epo-producing cells) and the tumours were in sites other than the kidney. As a result of these observations, Epo-TAgH mice were rendered chronically anaemic in order to increase the levels of SV40 T Ag in the kidney (Section 7.3.2). Under these circumstances there was expansion of the renal cell population. Infrequently, renal abnormalities were observed which probably represented a malignant transformation of the Epo-producing cells. In the absence of predictable malignant transformation in vivo, experiments were also undertaken to explant cells from the kidneys of Epo-TAg mice (Section 7.3.3). Although cell cultures were established succesfully, they did not express Epo-TAg or the native Epo gene. In Section 7.3.4 a different approach to obtaining renal Epo-producing cells was adopted. Conditionally transformed cell lines which had been characterised on morphological grounds as fibroblasts were exposed to hypoxia and assayed for Epo mRNA. In Section 7.3.5 the biology of Epo-producing cells was studied in the injured kidney. Different techniques were used to produce renal injury to one kidney and the effects on Epo expression in individual cells was assessed by labelling for SV40 T Ag. 
The aim of obtaining Epo-producing cells in culture has not yet been achieved. However, the experiments described in this chapter do give information on the behaviour of this cell population in response to a proliferative stimulus (expression of a viral oncogene), and in response to injury.

\subsection{METHODS}

\subsubsection{Post-mortem examination}

Mice found dead were examined post-mortem. Additionally, other mice were sacrificed because they were surplus to requirements or because of poor health (often excoriation). The kidneys, liver, gonads and chest cavity were examined; any abnormal organs were fixed by immersion in formol saline for microscopic examination. Macroscopic tumours were generally divided for immersion in formol saline, freezing in Cryo-M-Bed for cryosectioning, RNA extraction and on occasion for electron microscopy. Material for the latter was cut into fragments approximately $1 \mathrm{~mm}^{3}$ and fixed by immersion in $4 \%$ glutaraldehyde.

\subsubsection{Morphometry of the renal interstitium}

Kidneys for morphometric assessment were perfusion fixed in situ with $2 \%$ glutaraldehyde. The fixed kidney was then cut with a razor blade to yield segments of width $1 \mathrm{~mm}$ extending from the capsule to the inner medulla; these were embedded in epoxy resin. $1 \mu \mathrm{m}$ sections were cut and stained with Azure A.

\subsubsection{Explanting cells from kidneys for tissue culture}

Two general methods were employed in experiments to explant cells. In the first, the kidney was either flushed in situ by cannulation of the heart or directly excised. The tissue was then chopped finely and placed in a digestion medium containing collagenase, dispase and DNase. This was incubated (with or without stirring) at $37^{\circ} \mathrm{C}$. The crude preparation was then either strained and cultured on plastic dishes or separated by density gradient centrifugation using a Percoll gradient. In the second general method the kidney was diced with a razor blade and placed in a small amount of tissue culture medium in a sealed flask. After one week the medium and pieces of tissue were tipped off, the flask was rinsed and the remaining adherent cells were cultured by standard techniques. Medium was changed at approximately weekly intervals. Cultures usually underwent a period of crisis, following which a small number of colonies of growing cells survived. 


\subsubsection{Co-culture experiments}

A cell line (TG-L) isolated from the kidney of an Epo-TAg mouse was co-cultured with SV40 transformed proximal tubular cells in collaboration with Dr H. Muller. Two tubular cell lines (PKSV-PR and PKSV-PCT) derived from the pars recta and pars convoluta were used (kindly given by A. Vandewalle, Paris). First, TG-L cells were plated on to one side of $1 \mathrm{~cm}^{2}$ nucleopore membranes (pore size $0.1 \mu \mathrm{m}$ ) coated with rat tail collagen. After three days, when the TG-L cells were confluent, tubular cells were plated onto the other side of the membrane and grew to confluence overnight. The co-cultures were then incubated for 24 hours at $20 \%$ or $1 \%$ oxygen. RNA was then extracted by immersing the membrane in RNAzol B. $1 \mu \mathrm{g}$ of total RNA was reverse transcribed using a kit (Promega) and assayed for Epo mRNA by RT-PCR using primers located in exons 2 and 5. As a positive control RNA from hypoxic mouse kidney was analysed in parallel.

\subsubsection{Immunoprecipitation of SV40 $\mathrm{T}$ Ag}

In a single experiment to test whether immunoprecipitation and Western blotting would offer a more sensitive method for detecting SV40 T Ag, tissue or tissue culture cells were homogenised in 50mM Tris pH7.2, $5 \mathrm{mM} \mathrm{MgCl}_{2}, 1 \mathrm{mM} \mathrm{CaCl}_{2}, 10 \mathrm{mM}$ DTT, $1 \%$ NP40 with RNaseA, DNase 1 and protease inhibitors. $500 \mu \mathrm{g}$ total protein was incubated at $4^{\circ} \mathrm{C}$ with PAb419 (mAb to SV40 T Ag) overnight. Antibody was precipitated with Protein G sephadex, run on a polyacrylamide gel and transferred to Immobilon membrane. SV40 T Ag was then detected by incubation of the membrane with PAb423 (a second mAb to SV40 TAg) followed by goat anti-mouse Ig (DAKO). Bound antibody was detected by a chemiluminescent method (ECL, Amersham International)

\subsection{RESULTS}

\subsubsection{Effects of Epo-TAg expression in transgenic mice}

In order to assess the effects of expressing different levels of SV40 T Ag in the Epo-producing cells, a large colony of transgenic and non-transgenic mice was observed.

\subsubsection{Numbers of mice in the colony}

Table 7.1 summarises data concerned with the number of animals observed, and the age at death. To date, 564 Epo-TAg mice have died or have been sacrificed at ages greater than 6 months. 


\begin{tabular}{|c|c|c|c|c|}
\hline Line & Mode of death & $\mathrm{n}$ & $\begin{array}{l}\text { Mean age } \\
\text { (days) }\end{array}$ & $\begin{array}{l}\text { Range } \\
\text { (days) }\end{array}$ \\
\hline \multirow[t]{3}{*}{ Non Tg } & Natural & 26 & 350 & $33-576$ \\
\hline & Experimental & 152 & 270 & $31-617$ \\
\hline & Humane & 12 & 304 & $157-611$ \\
\hline \multirow[t]{3}{*}{175.5} & Natural & 2 & 126 & $34-217$ \\
\hline & Experimental & 33 & 285 & $73-574$ \\
\hline & Humane & 4 & 361 & $325-428$ \\
\hline \multirow[t]{3}{*}{176.5} & Natural & 8 & 242 & $29-410$ \\
\hline & Experimental & 14 & 189 & $78-497$ \\
\hline & Humane & 5 & 242 & $209-296$ \\
\hline \multirow[t]{2}{*}{$188.1 \mathrm{LC}$} & Natural & 1 & 336 & 336 \\
\hline & Experimental & 34 & 218 & $38-506$ \\
\hline \multirow{3}{*}{$188.1 \mathrm{HC}$} & Natural & 28 & 218 & $72-296$ \\
\hline & Experimental & 19 & 109 & $46-225$ \\
\hline & Humane & 12 & 209 & 101-299 \\
\hline \multirow[t]{3}{*}{188.3} & Natural & 2 & 326 & $312-340$ \\
\hline & Experimental & 18 & 323 & 53-638 \\
\hline & Humane & 3 & 317 & 286-337 \\
\hline \multirow[t]{3}{*}{$134.3 \mathrm{HC}$} & Natural & 8 & 300 & $190-404$ \\
\hline & Experimental & 6 & 163 & $63-383$ \\
\hline & Humane & 5 & 343 & $318-356$ \\
\hline \multirow{3}{*}{$\begin{array}{l}\text { Epo-TAg } \\
\text { heterozygote }\end{array}$} & Natural & 56 & 268 & 106-584 \\
\hline & Experimental & 265 & 210 & $38-625$ \\
\hline & Humane & 53 & 274 & $108-563$ \\
\hline \multirow{3}{*}{$\begin{array}{l}\text { Epo-TAg } \\
\text { homozygote }\end{array}$} & Natural & 22 & 225 & $22-330$ \\
\hline & Experimental & 110 & 207 & $35-412$ \\
\hline & Humane & 37 & 245 & $107-330$ \\
\hline
\end{tabular}

Table 7.1 - Ages of non-transgenic and Epo-TAg mice at death. Each of the transgenes was compatible with survival to an age of at least 10 months. Natural = found dead in cage; Humane = sacrificed on account of ill-health or development of a tumour; Experimental includes sacrifice for surveillance purposes.

Thus a large number of mice in the colony reached ages at which tumours could be expected. The data given also demonstrate that the Epo-TAg transgenes did not cause striking early mortality. The single exception to this was the $188.1 \mathrm{HC}$ line, in which a large number of animals died at about six months of age, usually with polycythaemia. In view of the relatively small numbers of mice in the groups, the diversity of the genetic background and the pathogens in the colonies (which included mouse hepatitis virus, Sendai virus and mites), statistical comparisons of the data in Table 7.1 have not been performed. In the $188.1 \mathrm{HC}$ line comparison of transgenic animals with non-transgenic littermates did demonstrate a significant reduction in lifespan (Table 7.2). 188.1HC mice were usually polycythaemic at the time of death and frequently had developed tumours overlying the mandible.

\begin{tabular}{|c|c|c|}
\hline Genotype & $\mathrm{n}$ & Age at death \\
\hline $188.1 \mathrm{HC}$ & 27 & $211+/-45$ \\
\hline Non TG & 28 & $391+/-138$ \\
\hline
\end{tabular}

Table 7.2 - The table shows natural survival of eleven litters of 188.1HC mice and their nontransgenic littermates. The age at death (days) is given as mean \pm s.d. The age at death is significantly different $(p<0.001)$. 


\subsubsection{Development of polycythaemia}

In three of the transgenic lines, $188.1 \mathrm{HC}, 134.3 \mathrm{HC}$ and 176.5 , polycythaemia was observed in older mice. This occurred at a later age than that seen in 198.3 mice (which bore the Epo$\mathrm{M}$ transgene), and was much more variable. Thus some of the Epo-TAg mice in these two lines maintained haematocrits within the normal range, whereas others developed severe polycythaemia (Table 7.3). Once the polycythaemia had developed it appeared to be progressive, again in contrast to that observed in 198.3 mice which was stable over time.

\begin{tabular}{|l|c|c|c|c|}
\hline \multicolumn{1}{|c|}{ Line } & Transgene & $\mathrm{n}$ & Mean haematocrit & Range \\
\hline Non tg & - & 43 & $41+/-5 \%$ & $26-49 \%$ \\
\hline 198.3 & Epo-M & 22 & $76+/-7 \%$ & $62-88 \%$ \\
\hline $188.1 \mathrm{HC}$ & Epo-TAg & 8 & $58+/-16 \%$ & $26-82 \%$ \\
\hline 176.5 & Epo-TAg & 17 & $69+/-16 \%$ & $44-90 \%$ \\
\hline
\end{tabular}

Table 7.3 - Haematocrits in mice of three lines in which mice became polycythaemic. Results are shown for mice older than 6 months. Whereas all 198.3 mice were polycythaemic at this age, the development of polycythaemia in $188.1 \mathrm{HC}$ and 176.5 mice was sporadic.

Initially it seemed likely that polycythaemia in these mice was caused by an expansion of the Epo-producing cells in the kidney, secondary to SV40 T Ag expression. However, no evidence of Epo-TAg expression was found on immunostaining kidneys from polycythaemic mice. It remained possible that Epo-TAg expression was suppressed by the polycythaemia. To examine this, a polycythaemic animal was rendered anaemic by recurrent venesection. The kidney still did not contain SV40 T Ag. Neither Epo nor Epo-TAg mRNA were present on RNase protection analysis of total RNA from the liver and kidney of polycythaemic mice; nor were they present in these organs when the haematocrit was reduced to normal levels. This demonstrated that the polycythaemia was not due to renal Epo production. This is further supported by the observation that polycythaemia did not occur in those lines which expressed the highest renal levels of Epo-TAg (Epo-TAgH, $188.1 \mathrm{LC}$ and 188.3).

In some animals with polycythaemia, infiltrating cells (bearing LCA) were observed in the renal interstitium. This raised the possibility that the renal cells expressing Epo-TAg were being destroyed by an autoimmune process. An immune reaction to SV40 T Ag has been observed in pancreatic islets of mice expressing SV40 T Ag under control of the insulin promoter (Adams et al. 1987). To test for antibodies to SV40 T Ag, serum from a mouse with pronounced infiltration was incubated with acetone-fixed Cos 7 cells (which express SV40 T Ag), followed by peroxidase-conjugated anti-mouse Ig. No labelling was observed. Subsequently, such infiltrates were observed in non-transgenic mice and are presumed to represent a non-specific finding in elderly mice. 
The polycythaemia thus appears to be due to extrarenal Epo production, and not to an expansion of Epo producing cells in the kidney. Subsequently, studies in homozygous Epo-TAgH mice (Chapter 8) conclusively demonstrated that transcripts can run through the SV40 polyadenylation signals into Epo sequence, and that immunoreactive Epo can be produced by the Epo-TAg gene. Therefore it is likely that the polycythaemia in 134.3HC, 176.5 and $188.1 \mathrm{HC}$ mice is due to Epo from the Epo-TAg transgene at an extrarenal site (rather than the native Epo gene). Whether this involves expansion of a cell population, and whether it reflects any physiological aspect of Epo production is not known. That the development of polycythaemia was idiosyncratic in contrast to preputial gland enlargement or the development of polycythaemia in 198.3 mice suggests that an expansion or transformation of a cell population might be required.

\subsubsection{Preputial gland enlargement}

The most striking phenotypic observation was the enlargement of the preputial gland in male Epo-TAg transgenic mice of all lines except for 99.5 and Epo-TAgH. This became clear in mice of these lines at around the time of sexual maturity, and was invariably present. An example of preputial gland enlargement is shown in Figure 7.1. The preputial gland is a modified sebaceous gland and is important in pheromone secretion in rodents. An important question raised by this striking observation was whether the preputial gland was a site of Epo expression. This was demonstrated to be the case (see Chapter 4). Any physiological significance of Epo production by this gland remains unknown. The increase in size of the preputial gland did not progress to a malignant tumor in any mouse. The increase in size of the gland could have been due to direct expansion of a cell population (eg the secretory epithelium) or could have been mediated by an increase in the levels of growth factors. Alternatively, it is possible that the expansion was due to an increase in the viscosity of the secreted fluid. Immunostaining for SV40 T Ag demonstrated that the cells expressing EpoTAg were interstitial cells, lying between the epithelial structures (Figure 7.1B). 


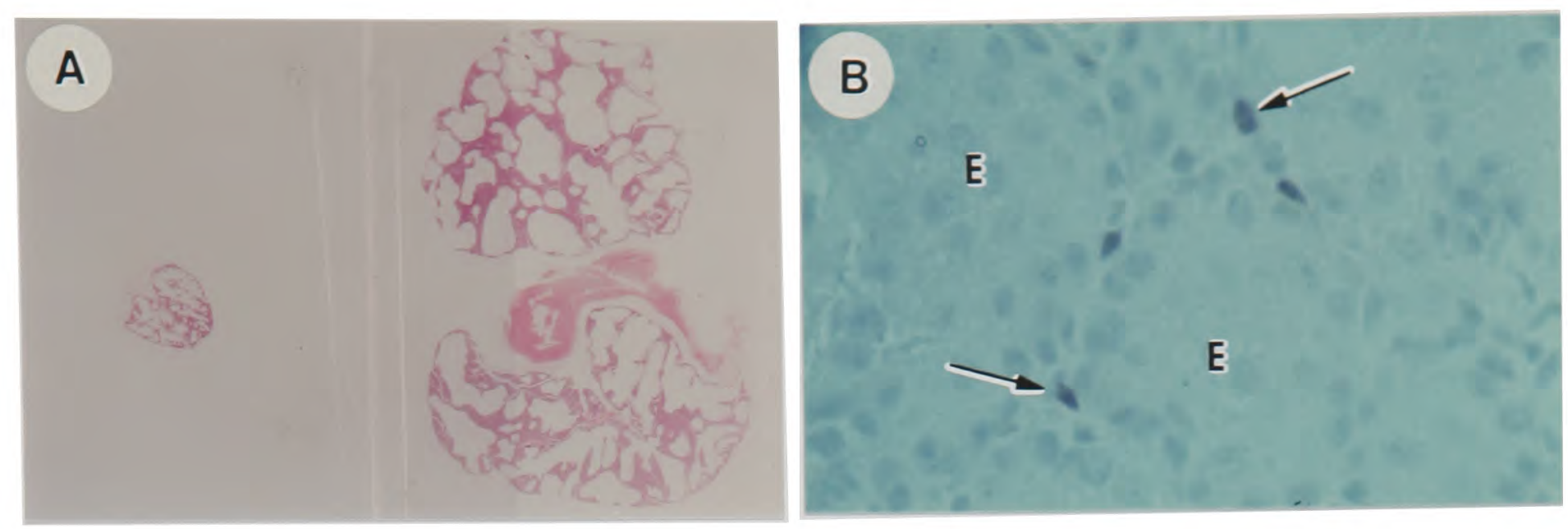

Figure 7.1 - A. Preputial gland from a non-transgenic mouse (left) and a 176.5 transgenic mouse (right). The transgenic mouse has an enlarged gland with thickened parenchyma. Magnification $x 2$.

B. Preputial gland from an Epo-TAg ${ }^{H}$ homozygote rendered acutely anaemic with phenylhydrazine. Cryostat section stained for SV40 T Ag. Positively labelled nuclei (arrows) are observed lying between the epithelial structures (E). Magnification $x 450$.

The normal size of the preputial gland in heterozygous Epo-TAgH mice suggested that SV40 T Ag in this line was inactive. This could have been due to a mutation in the viral sequence at the time of the homologous recombination giving rise to Epo-TAgH. Because of this concern the initial mouse colony observed for the development of renal tumours was composed of animals bearing other Epo-TAg transgenes.

\subsubsection{Development of tumours in the colony}

Tumours were observed in significantly more mice expressing the Epo-TAg transgene than in controls, as summarised in Table 7.4. The control animals had a significant incidence of lymphoid tumours; similar tumours were observed in transgenic mice and did not contain SV40 T Ag. It was anticipated that Epo-TAg transgenic mice, in which the predominant site of transgene expression was the kidney, would develop renal tumours containing SV40 T Ag. However, almost all tumours observed were in sites other than the kidney.

\begin{tabular}{|l|c|c|c|c|c|c|c|}
\hline \multicolumn{1}{|c|}{ Line } & $\mathrm{n}$ & Tumors & Kidney & Liver & Lymph $^{1}$ & Jaw & Other \\
\hline Control & 250 & $3.2 \%$ & $0.4 \%$ & $0.4 \%$ & $2.0 \%$ & - & $0.4 \%^{2}$ \\
\hline $188.1 \mathrm{HC}$ & 44 & $22.7 \%$ & - & - & - & $18.1 \%$ & $4.5 \%^{3}$ \\
\hline $134.3 \mathrm{HC}$ & 16 & $25.0 \%$ & - & $6.2 \%$ & - & $25.0 \%$ & - \\
\hline 175.5 & 30 & $3.3 \%$ & - & $3.3 \%$ & - & - & - \\
\hline 176.5 & 28 & $32.1 \%$ & - & - & - & $28.6 \%$ & $3.6 \%{ }^{4}$ \\
\hline 188.3 & 19 & $15.8 \%$ & - & $10.5 \%$ & $5.2 \%$ & - & - \\
\hline $188.1 \mathrm{LC}$ & 21 & $4.8 \%$ & - & - & - & $4.8 \%$ & - \\
\hline
\end{tabular}

Table 7.4 - Incidence of tumours in the mouse colony. Mice dying at ages greater than 155 days are included. Controls include non transgenic mice, Epo-M mice, and the 99.5 line which did not express the Epo-TAg transgene. Some mice had tumours at more than one site. The transgenic animals have been ranked in ascending order of the renal expression of EpoTAg (i.e. lowest in 188.1HC and highest in 188.1LC). It would be anticipated that tumours due to faithful expression of Epo-TAg would increase further down the table. Notes: 'Lymphomas included anterior mediastinal masses, diffusely enlarged lymph nodes and infiltration of the kidneys, liver and spleen. Other sites as follows; ${ }^{2}$ testis, ${ }^{3}$ base of brain, ${ }^{4}$ prostate. 
Many of the excess tumours in Epo-TAg mice were observed over the jaw. These tumours were sporadic; but quite frequent. Considerable numbers of mice from each line did not develop such tumours, suggesting that another factor, either genetic or environmental, was involved in tumourogenesis. An example is shown in Figure 7.2. The tumours were located over the jaw, and could reach $1 \mathrm{~cm}$ diameter. They were clearly attached to the bone, and the muscles of the cheek appeared to be stretched over the tumour. Microscopically they were invasive, poorly differentiated and appeared to be forming bone. On immunostaining, these tumours contained SV40 T Ag (Figure 7.2). RNase protection demonstrated the presence of Epo-TAg mRNA, but not of Epo mRNA. Malignant tumours containing SV40 $\mathrm{T}$ Ag were also observed at the base of the brain. Although it is possible that these tumours involving bone were metastases no distant primary tumour was found in any mouse.

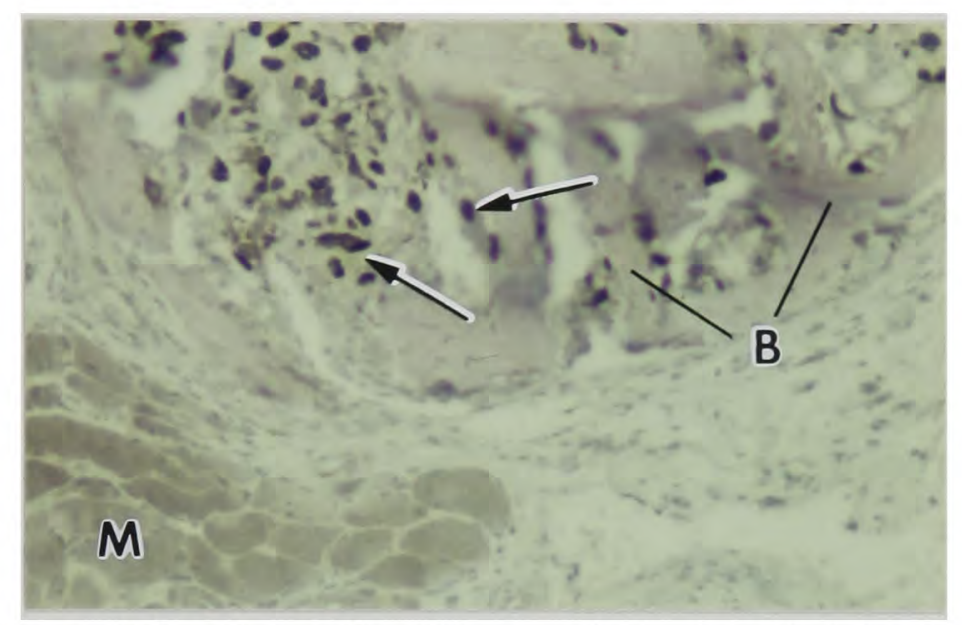

Figure 7.2 - Tumour overlying the angle of the mandible, labelled for SV40 TAg. The tumour cells have positively labelled nuclei (black, arrows) and can be seen to be invading the bone (B). Muscle (M) lies adjacent to the tumour. Magnification $x 250$.

Single or multifocal tumours in the liver were quite frequent, and were usually associated with weight loss or were found at post-mortem. An example is shown in Figure 7.3A. Tumour cells stained positive for SV40 T Ag. The cells were pleomorphic and undifferentiated. They contained areas of bone. When stained by von Kosser's method, they contained calcified areas and probable osteoid (Figure 7.3B). These hepatic tumours would therefore be classified as osteosarcomas. Although there were frequently multiple tumour nodules, suggesting that they might be metastatic, no primary tumour outside the liver was found. The observation of liver tumours was of particular interest in view of the expression of the Epo gene in normal liver. However, these tumours did not bear any clear relation to either Ito cells or hepatocytes. 


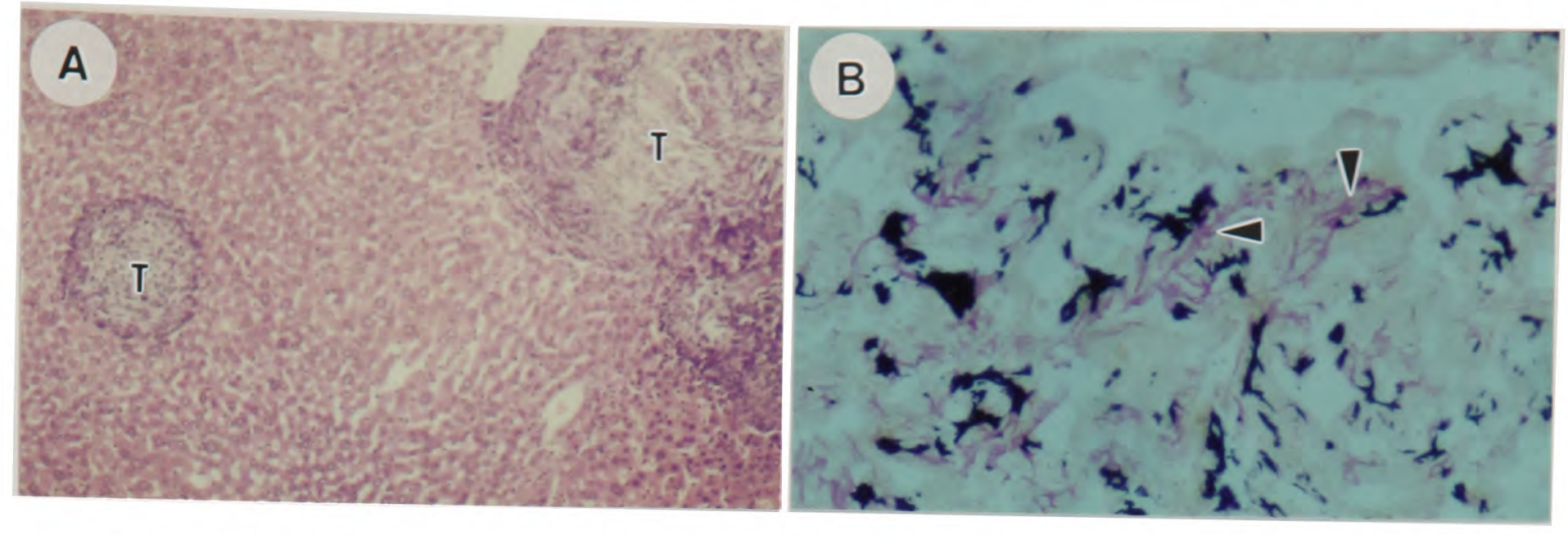

Figure 7.3 - A. Liver tumour, stained with $H \& E$. The nodules of tumour $(T)$ are relatively basophilic. Magnification $x 100$.

$B$. Liver tumour stained by von Kosser's method, showing extensive calcification (black) and probable osteoid formation (pink, arrowheads). Magnification $x 400$.

The presence of SV40 T Ag strongly suggests that expression of Epo-TAg is causally involved in the development of these tumours. Analysis of the distribution of Epo-TAg mRNA in these lines of Epo-TAg mice had demonstrated that the level of expression in the kidney was relatively low, and that there was over-expression at other sites (Chapter 4). Consequently, it is perhaps not unexpected that these mice developed tumours at extrarenal sites.

\subsubsection{Observation of Epo-TAg ${ }^{H}$ mice}

Following these initial studies of Epo-TAg mice, two observations demonstrated that the SV40 T Ag produced by the Epo-TAg ${ }^{H}$ gene was active. Firstly, Epo-TAgH homozygotes were bred and had preputial gland enlargement. The other Epo-TAg lines over-expressed the Epo-TAg gene in the preputial gland in comparison to the native gene and this presumably accounts for the marked difference in gland size between them and the Epo-TAgH heterozygotes. Secondly, some tumours bearing SV40 TAg were observed in heterozygous Epo-TAgH mice; furthermore one of these tumours was in the kidney. The Epo-TAgH line, which expressed the gene at a higher level in the kidney than the other Epo-TAg lines (Chapter 4), offered the possibility of improved precision of delivery of SV40 T Ag to the appropriate renal cells. However, in Epo-TAg ${ }^{\mathrm{H}}$ mice tumours were again predominantly in other organs (Table 7.5).

\begin{tabular}{|l|c|c|c|c|c|c|c|c|}
\hline Animals & $\mathrm{n}$ & Tumors & Kidney & Liver & Lymph & Eye & Jaw & Other \\
\hline Control & 250 & $3.2 \%$ & $0.4 \%$ & $0.4 \%$ & $2.0 \%$ & - & - & $0.4 \% 1$ \\
\hline $\begin{array}{l}\text { Epo-TAg } \\
\text { hetero }\end{array}$ & 175 & $14.9 \%$ & $0.6 \%$ & $6.3 \%$ & $4.0 \%$ & $1.2 \%$ & $2.3 \%$ & $1.2 \% 1,2$ \\
\hline
\end{tabular}

Table 7.5 - The incidence of tumours in heterozygous Epo-TAg ${ }^{H}$ mice. Mice dying at ages greater than 155 days are included. The controls are as in Table 7.4. 'Testis, ${ }^{2}$ Intestinal. 
Liver tumours of similar morphology to those seen in other Epo-TAg mice were not infrequent. One of the tumours in the liver was of a different morphology, consisting of well-differentiated spindle cells, without any evidence of synthesis of bone (Figure 7.4). In addition to tumours in the liver, tumours were observed over the jaw and two tumours occurred just below the eye. Electron microscopy of the tumours related to the eye showed the presence of viral particles (Figure 7.5); whether these were incidental to tumour formation is not known. Such viral particles were not observed in any kidney or liver specimens from any mouse examined at the ultrastructural level for the experiments in Chapters 5 and 6.

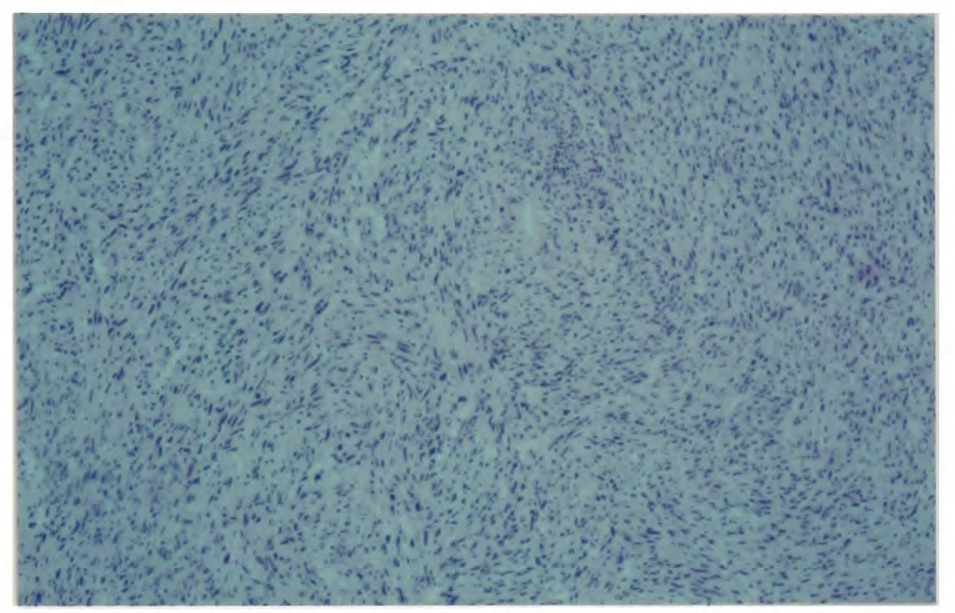

Figure 7.4 - Spindle cell tumour from the liver, stained with H\&E. Stellate shaped tumour cells are seen; there was no evidence of calcification in this tumour. Magnification $x 100$.

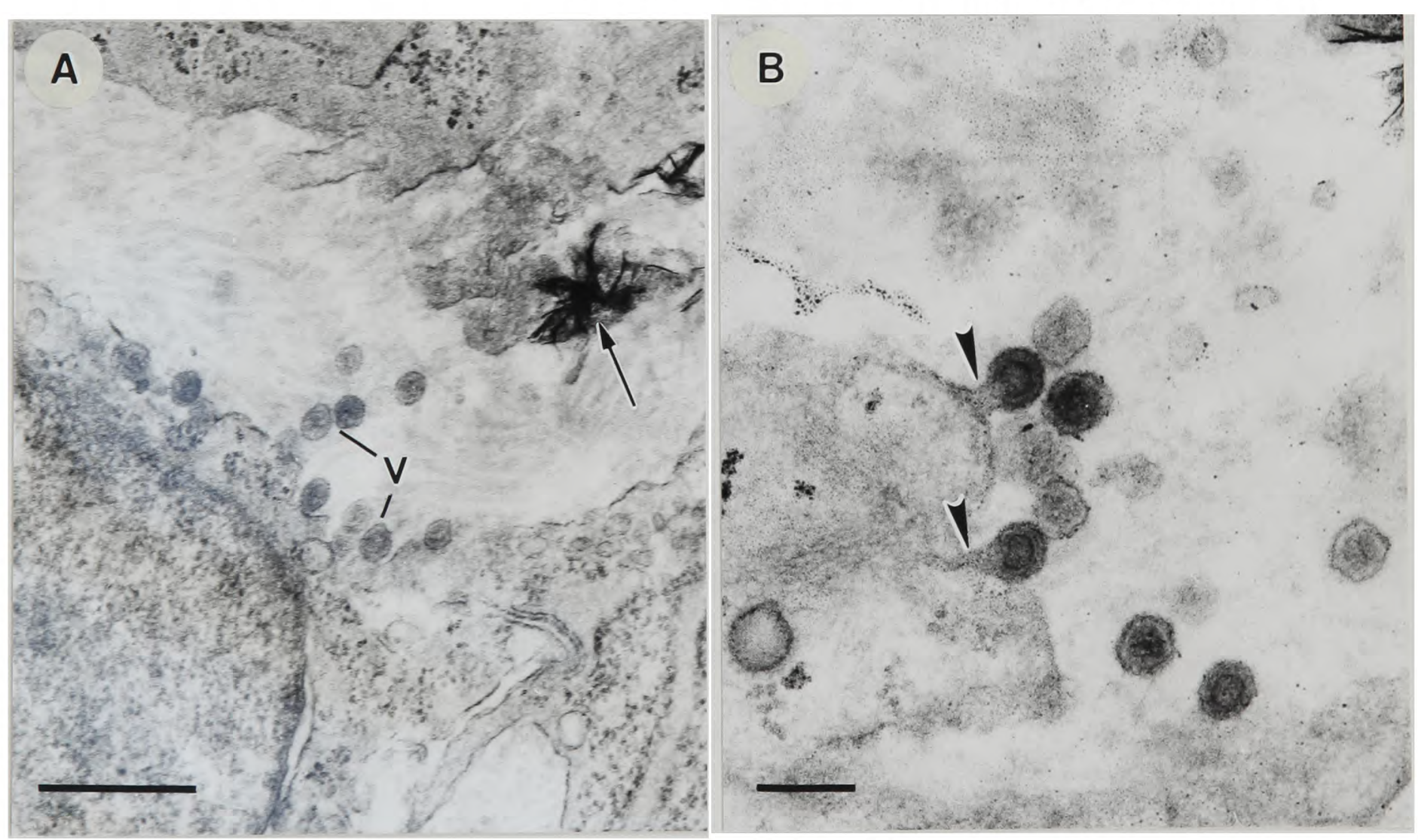

Figure 7.5 - Electron micrographs of a tumour adjacent to the orbit.

$A$. There is a region of calcification (arrow). Virus particles are seen (V). Bar is $0.5 \mu \mathrm{m}$.

$B$. Higher power view showing viral particles budding from the cell surface (arrowheads).

Bar is $0.2 \mu \mathrm{m}$.

RNA was extracted from a small number of these tumours from Epo-TAgH mice. The tumours contained Epo-TAg mRNA, but not Epo mRNA. This observation of expression of the Epo-TAg gene, but not the Epo gene is not explained. Since it appears that there is no alteration to the surrounding DNA in the Epo-TAg ${ }^{H}$ recombinant gene it is surprising. Several 
explanations for this could be offered. One is that in the tumours the Epo-TAg gene is amplified or modified. Another possibility is that the effect is due to the insertion of the SV40 T Ag coding sequence. For instance the $2.7 \mathrm{~kb}$ sequence employed includes binding sites for SV40 T Ag and could alter transcription of the fusion gene. Similarly, the difference in expression might be due to stabilisation of Epo-TAg mRNA in some cells where Epo mRNA is not stable.

The development of tumours at various sites outside the kidney is of some interest. How tumours at these sites relate to the capacity for Epo gene expression remains uncertain. The observation of these tumours in the Epo- $\mathrm{TAg}^{\mathrm{H}}$ mice makes it more likely that they are related to a genuine capacity for Epo gene expression in these tissues. Tumours at unexpected sites have occurred in other transgenic mice expressing SV40 T Ag under the control of tissue-specific elements (Skalknik et al. 1991; Ceci et al. 1991; Montag et al. 1993). In one of these studies the use of flanking sequence from a myelomonocytic-specific gene to direct SV40 TAg expression resulted in the development of neuroblastoma in the prostate in addition to haematological malignancies (Skalknik et al. 1991). In a manner similar to the current studies the tumours expressed the transgene, but not the native gp91-phox gene.

One important conclusion is that the SV40 T Ag produced by the Epo-TAg and Epo-TAgH genes is capable of inducing tumour formation at a high rate, and of inducing striking abnormalities in the preputial gland. The low rate of tumours in the kidney is unlikely to be due to any mutation in the SV40 T Ag coding sequence.

\subsubsection{Renal tumour in an Epo-TAg ${ }^{H}$ mouse}

A single kidney tumour was observed in a heterozygous Epo-TAgH mouse. This mouse was polycythaemic (haematocrit $65 \%$ ), in contrast to other Epo-TAg ${ }^{\mathrm{H}}$ mice with tumours where the haematocrit was measured ( $\mathrm{n}=5$, mean $24 \%$, range $17-32 \%)$. In fact polycythaemia was not observed in any other Epo-TAgH mouse at any age $(n=307$, mean $36 \%$, s.d. $\pm 4.7 \%$, range 20-49\%). This suggests that the tumour may have produced Epo. Immunostaining demonstrated the presence of SV40 T Ag in the tumour, and expression of 5'NT. The tumour cells did not label with antibodies to cytokeratin, desmin, smooth muscle actin, PECAM/CD31 or LCA. Neither did the tumour label with ER-TR7. However, the anticytokeratin antibody did not label all mouse cytokeratins (many tubular cells did not label in cryostat sections of kidney). Furthermore, tubular cells bear 5 NT. It therefore remains 
possible that the origin of this tumour was epithelial. On RNase protection analysis, neither Epo-TAg or Epo transcripts were detected. Possibly the expression in the tumour was regulated, and in the presence of polycythaemia or cytokines had been down-regulated.

Cells from this kidney have subsequently been studied in tissue culture. In the cultures, several different cellular morphologies have been observed. Some cells stained positively for 5'NT and some for smooth muscle actin. However, on hypoxic exposure for 72 hours no Epo was detected in the culture medium by RIA, and after 16 hours hypoxic exposure no Epo mRNA or Epo-TAg mRNA was detectable by RNase protection.

That this tumour occurred in an animal previously given phenylhydrazine may be relevant. The haemolysis may have been severe enough to injure the kidney and deliver a second proliferative stimulus to the Epo-producing cells. Subsequently a further cohort of heterozygous Epo-TAgH animals have been given phenylhydrazine and are currently under observation. The rest of the kidney in this animal was not normal at the time of sacrifice; it had cystic areas, dilated tubules and interstitial expansion. There was evidence of amyloid deposition in the liver, and to a lesser extent in the kidney. These abnormalities may have been important in the development of this tumour; however amyloid occurs quite commonly in some strains of mice (Russell and Meier 1966) and may have represented an incidental finding.

Thus, although the SV40 T Ag produced by the Epo-TAgH gene was capable of producing tumours, the incidence of renal tumours was very low. Therefore strategies were adopted to increase the level of Epo-TAgH expression in the kidney. These were combined with morphometric assessment of the renal interstitium, to determine whether sustained, highlevel expression of Epo-TAgH would have any effect on the Epo-producing cells.

\subsubsection{Strategies to increase levels of SV40 T Ag in the renal interstitium in Epo- TAgH mice}

In order to further increase the level of SV40 T Ag in the renal Epo-producing cells, chronic anaemia was used to induce renal expression in Epo-TAgH mice. This was achieved by three methods.

(1) Crosses with Nan mice. Nan mice were obtained from the MRC Radiobiology Unit, Harwell. Affected mice have a haemolytic anaemia which is transmitted as an autosomal dominant trait (Lyon et al. 1983). The mice are maintained by breeding identified males (which are pale at birth) with an $\mathrm{F}_{1}$ cross $(\mathrm{C} 3 \mathrm{H} / \mathrm{HeH} \times 101 / \mathrm{J})$. Four males were obtained, 
and were crossed with Epo-TAg ${ }^{\mathrm{H}}$ transgenic mice. Identification of mice with the haemolytic anaemia was made either on the basis of pallor at birth, low haematocrit relative to littermates at the time of tail biopsy, or splenomegaly at post-mortem. Nan mice maintain a haematocrit of approximately 35\%. Epo-TAgH mice which were crossed with Nan and selected for pallor at birth had a haematocrit of $25-30 \%$.

(2) Iron deficiency anaemia. Three litters which were offspring of Epo-TAg ${ }^{\mathrm{H}}$ mice were placed on an iron-deficient diet at the time of weaning; these included nine transgenic and eight non-transgenic mice. The mice were intermittently venesected to exacerbate the iron deficiency. The haematocrits obtained are shown in Figure 7.6, plotted against age.

(3) Homozygous Epo-TAg ${ }^{\mathrm{H}}$ mice. Epo-TAg ${ }^{\mathrm{H}}$ mice were bred to homozygosity, and found to be viable, to develop normally and to be fertile. In terms of targeting delivery of SV40 T Ag these mice were attractive, since they have a chronic anaemia (Hct 12-25\%) and have two copies of the homologously inserted Epo-TAg gene. The principle disadvantage, in terms of developing cell culture lines is that they have no normal Epo gene. This is of some concern in view of the observation that the Epo-TAgH gene can behave differently from the Epo gene, since in the liver the expression of the latter is relatively reduced (Chapter 6), and in some tumours from heterozygous Epo-TAgH mice expression of Epo-TAgH but not Epo was observed (see above).

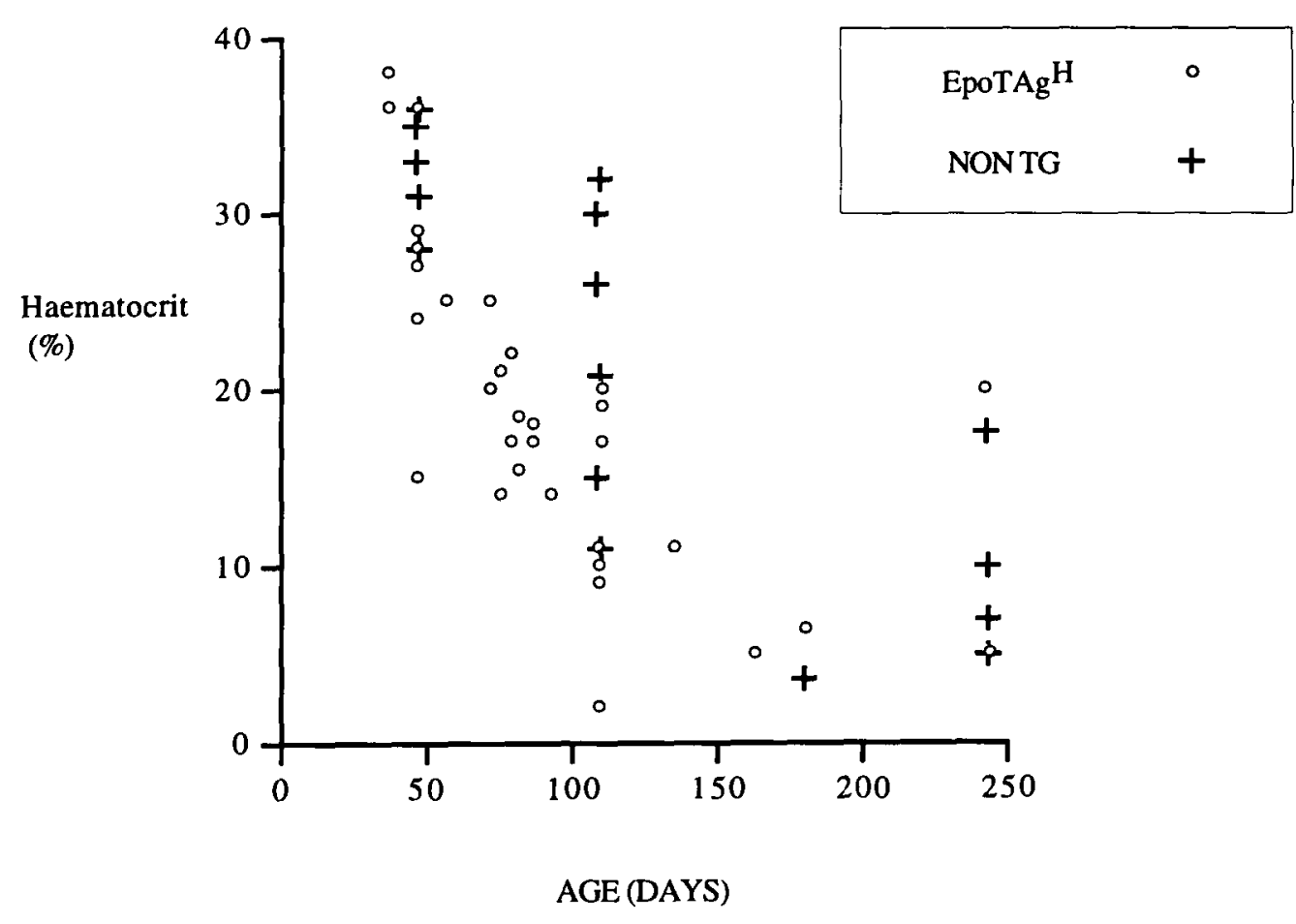

Figure 7.6 - Haematocrits of mice placed on an iron deficient diet at the time of weaning, with additional venesection. Haematocrits were generally lower in the Epo-TA $g^{H}(o)$ than in the non-transgenic mice (+); however, severe anaemia was produced in both groups of mice. 


\subsubsection{Effects of high level SV40 T Ag expression in the renal interstitium}

As described in Chapter 4, the number of renal interstitial cells in 3 month old heterozygous Epo-TAgH mice was not significantly different from the number in controls. The effect of longer term, high level expression of the transgene was now studied. By analogy with similar experiments (Hanahan 1985; Ornitz et al. 1987), it was anticipated that the cell population expressing SV40 T Ag would expand as a prerequisite to tumour development. Homozygous Epo-TAgH mice were studied at the age of 8 months, and compared with nontransgenic littermates. Morphometric assessment was performed on Azure A-stained $1 \mu \mathrm{m}$ resin sections, facilitating assessment of the interstitium. In this material there was a striking increase in the number of nuclei and the area of the interstitium (Table 7.6 and Figure 7.7).

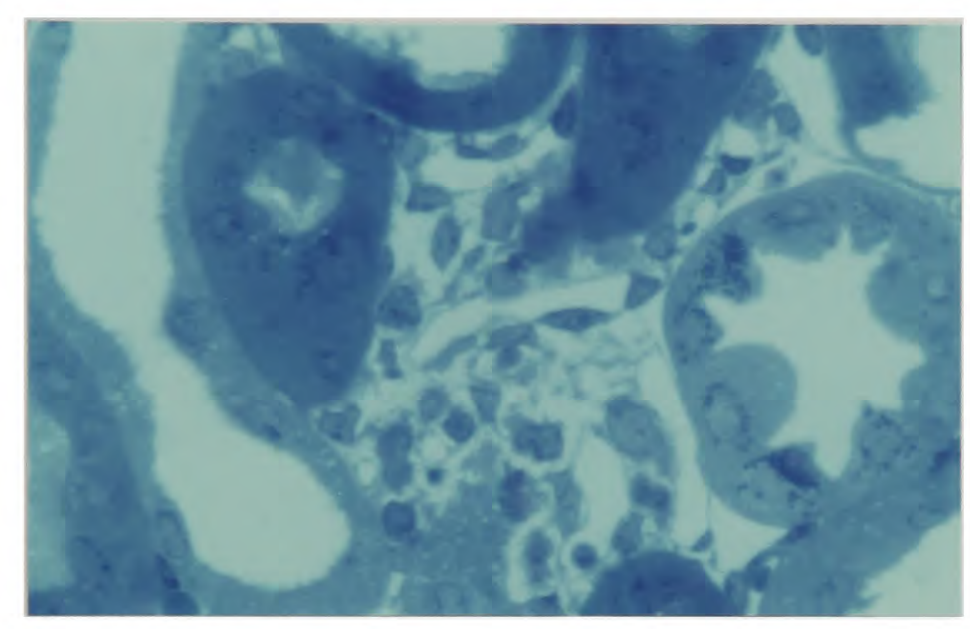

Figure 7.7 - Expansion of the interstitial cell population in an Epo-TAg ${ }^{H}$ homozygote. Epoxy resin $1 \mu \mathrm{m}$ section stained with Azure A showing an expanded interstitial space with an increased number of nuclei. Magnification $x 500$.

\begin{tabular}{|c|c|c|}
\cline { 2 - 3 } \multicolumn{1}{c|}{} & Controls & Epo-TAg ${ }^{\mathrm{H}}$ homozygotes \\
\hline superficial cortex & $1696+/-320$ & $2816+/-480$ \\
\hline mid-cortex & $1648+/-256$ & $2672+/-288$ \\
\hline deep cortex & $1776+/-320$ & $3168+/-240$ \\
\hline
\end{tabular}

Table 7.6 - The effect of long-term expression of SV4O T Ag in interstitial cells. The table indicates the number of interstitial nuclei per $\mathrm{mm}^{2}$ in the cortex of kidneys from 8 month old Epo-TAg ${ }^{H}$ homozygotes and non-transgenic controls (mean $\pm s . d$. of nine values, three from each animal). In the chronically anaemic transgenic animals there is a statistically significant increase in the number of interstitial cells in each region of the cortex $(p<0.01)$.

An important caveat in interpreting this data is that anaemia has been demonstrated to increase interstitial volume in the rat (Kaissling et al. 1992). Therefore it was possible that the interstitial expansion was directly due to anaemia, and was unrelated to SV40 T Ag expression. Since anaemia or hypoxia is required to induce Epo-TAg expression in the transgenic mice this is a difficult issue to address experimentally. However, this has been achieved by studying the effects of iron deficiency anaemia in non-transgenic and Epo$\mathrm{TAg}^{\mathrm{H}}$ heterozygous mice. In these experiments both groups of mice developed a severe and sustained anaemia. Morphometry on perfusion-fixed material demonstrated that the 
number of cortical interstial cells was substantially higher in the transgenic mice than in their non-transgenic littermates (Table 7.7), although the mean haematocrit was the same (6.5\%).

\begin{tabular}{|c|c|c|}
\cline { 2 - 3 } \multicolumn{1}{c|}{} & Controls & Epo-TAg $^{\mathrm{H}}$ \\
\hline superficial cortex & $2523+/-315$ & $4501+/-430$ \\
\hline mid-cortex & $2411+/-349$ & $4264+/-397$ \\
\hline deep cortex & $2235+/-379$ & $4845+/-349$ \\
\hline outer stripe & $2157+/-338$ & $4008+/-471$ \\
\hline
\end{tabular}

Table 7.7 - The effect of long-term expression of SV4O T Ag in interstitial cells. The table indicates the number of interstitial nuclei per $\mathrm{mm}^{2}$ in the cortex of kidneys from 6 to 12 month old iron deficient Epo-TAg ${ }^{H}$ heterozygotes and iron deficient non-transgenic controls (mean $\pm s . d$. of six values, three from each animal). In the chronically anaemic transgenic animals there is a statistically significant increase in the number of interstitial cells in each region of the cortex $(p<0.01)$. These morphometric analyses were performed on $3 \mu \mathrm{m}$ paraffin sections; they are not directly comparable to those in Table 7.6.

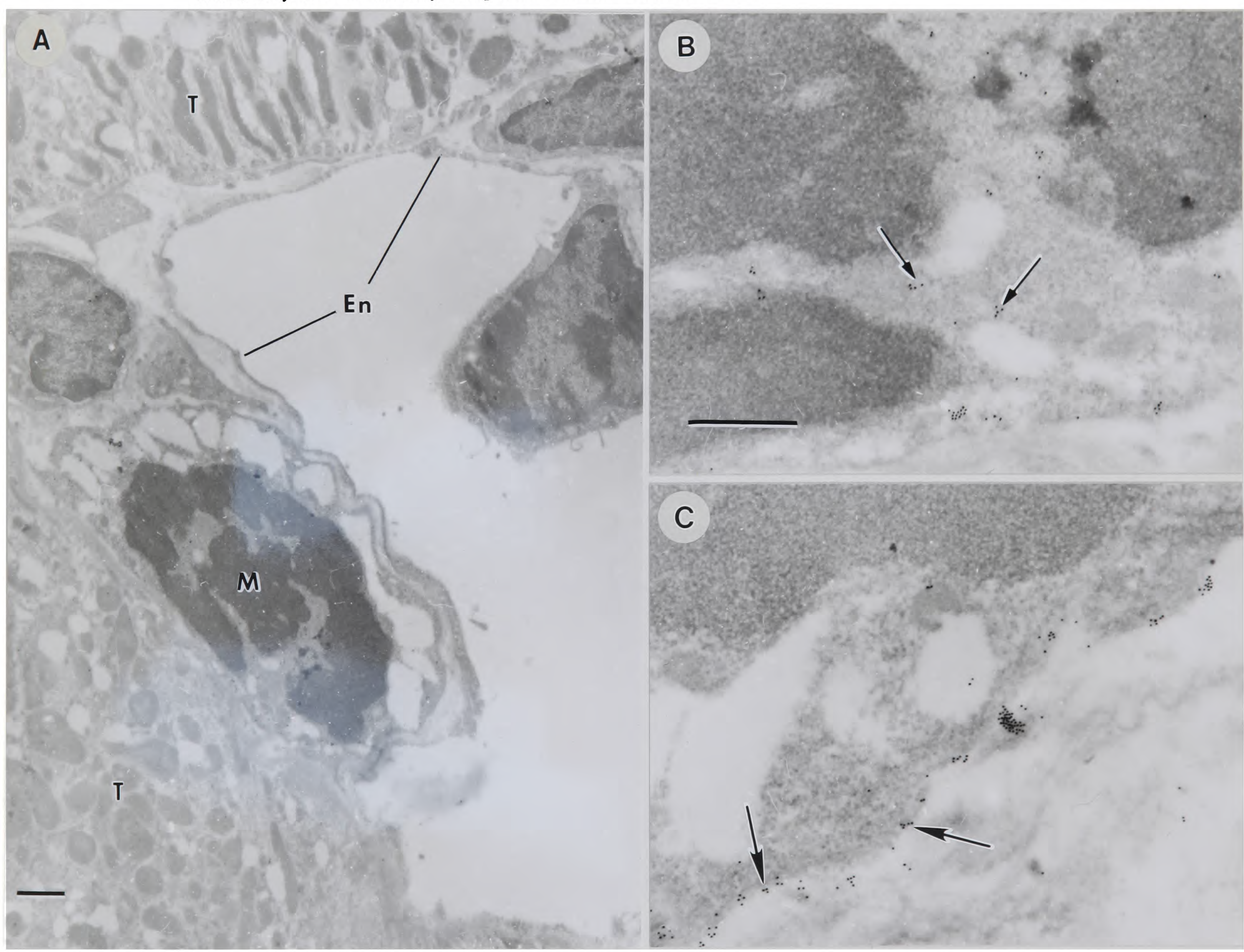

Figure 7.8 - A. Electronmicrograph of an interstial cell undergoing mitosis (M). Adjacent endothelium (En) and tubular cells (T) are indicated. Bar is $0.5 \mu \mathrm{m}$.

$B$. Immunolabelled for SV $40 \mathrm{TAg}$ showing gold particles (arrows) over the electron-lucent region of the nucleus. Bar is $0.2 \mu \mathrm{m}$.

$C$. Adjacent section labelled for 5'NT showing gold particles (arrows) along the plasmalemma. Magnification as in $B$. 
Thus high-level expression of SV40 T Ag leads to a predictable expansion of the interstitial cells. We may conclude that cells expressing Epo-TAg (and presumably Epo) are not terminally differentiated. Further evidence for this was the observation of a cell containing $\mathrm{T}$ Ag undergoing mitosis. This cell was also shown to bear 5'NT on its surface (Figure 7.8).

\subsubsection{Development of tumours}

Having demonstrated that these strategies to increase SV40 T Ag expression could result in expansion of the renal interstitium, a large number of anaemic Epo-TAg ${ }^{\mathrm{H}}$ mice were observed for tumour development (Table 7.8).

\begin{tabular}{|l|c|c|c|c|c|c|c|c|}
\hline Animals & $\mathrm{n}$ & Tumors & Kidney & Liver & Lymph & Eye & Jaw & Other \\
\hline Control & 250 & $3.2 \%$ & $0.4 \%$ & $0.4 \%$ & $2.0 \%$ & - & - & $0.4 \% 1$ \\
\hline $\begin{array}{l}\text { Epo-TAg } \\
\text { hetero }\end{array}$ & 175 & $14.9 \%$ & $0.6 \%$ & $6.3 \%$ & $4.0 \%$ & $1.2 \%$ & $2.3 \%$ & $1.2 \% 1,2$ \\
\hline $\begin{array}{l}\text { Epo-TAg } \\
\text { x Nan }\end{array}$ & 54 & $11.1 \%$ & - & $3.7 \%$ & $3.7 \%$ & $3.7 \%$ & - & - \\
\hline $\begin{array}{l}\text { Epo-TAg } \\
\text { Low Fe }\end{array}$ & 10 & $10.0 \%$ & - & - & - & $10.0 \%$ & - & - \\
\hline $\begin{array}{l}\text { Epo-TAg } \\
\text { Homo }\end{array}$ & 125 & $11.2 \%$ & $8.0 \%$ & $1.6 \%$ & $0.8 \%$ & $0.8 \%$ & $0.8 \%$ & - \\
\hline
\end{tabular}

Table 7.8 - Effect of chronic anaemia on the incidence of tumours in Epo-TAg ${ }^{H}$ mice. Anaemia was generated in three ways; the degree and duration of anaemia would be expected to be more severe lower down the table. The data in the first two rows of the table is as given in Table 7.4. ' Testis, ${ }^{2}$ Intestinal

Renal tumours were not observed in the Nan crosses or the iron-deficient mice. The tumours at other sites were similar to those in other Epo-TAgH heterozygotes. However, in contrast to other Epo-TAg mice renal abnormalities were observed in approximately $8 \%$ of homozygous Epo-TAg ${ }^{\mathrm{H}}$ mice reaching the age of six months. Macroscopically the kidneys were enlarged with pale nodules or cysts. In the abnormal regions there was a greatly expanded interstitium, containing tightly packed cells with little cytoplasm (Figure 7.9A). Cystic structures, lined by epithelial cells, were also frequently observed. One of these kidneys has been studied in detail. The majority of the interstitial cells in the abnormal region of the kidney expressed nuclear SV40 T Ag. There was extensive staining with ERTR7 and 5'NT in this region. In double-labelling studies with 5'NT and SV40 T Ag some cells were double-labelled, but other cells were labelled only for one or other antigen (Figure 7.9B). It is attractive to hypothesise that Epo-TAg was only being expressed in hypoxic regions; however this remains unproved. There was a moderate increase in cells bearing CD45 (leucocyte lineage) and F4/80 (macrophage) in the abnormal region. No double 
staining for SV40 T Ag and these markers was observed. Capillary density was somewhat decreased compared to normal interstitium, as assessed by labelling for CD31/PECAM; there was no double-labelling for SV40 T Ag and this marker.
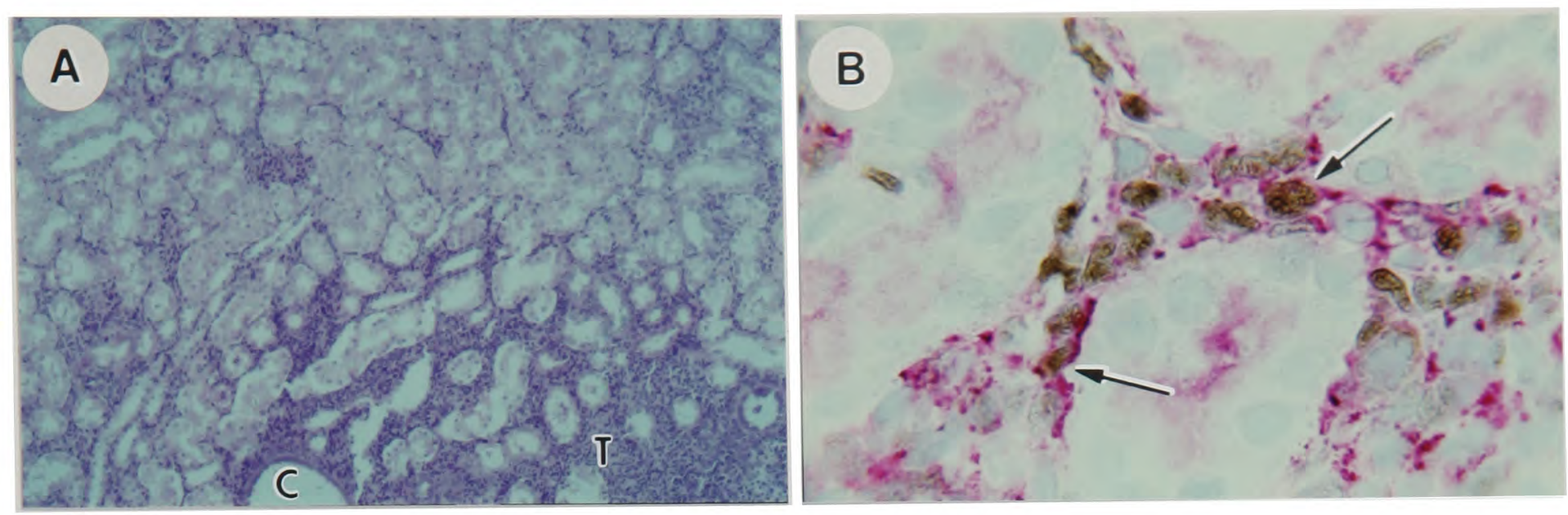

Figure 7.9 - Interstitial cell "tumour" in an Epo-TAg ${ }^{H}$ homozygote.

A. H\&E stained section. In the abnormal region $(T)$ the interstium is expanded and packed with cells. A cyst $(C)$ is seen. Magnification $x 125$.

B. Cryostat section of a region from this "tumour" labelled for SV4O TAg (brown, nuclear) and 5'NT (red, cell surface). Some of the cells with nuclear TAg bear 5'NT (arrows); others do not. Magnification $x 450$.

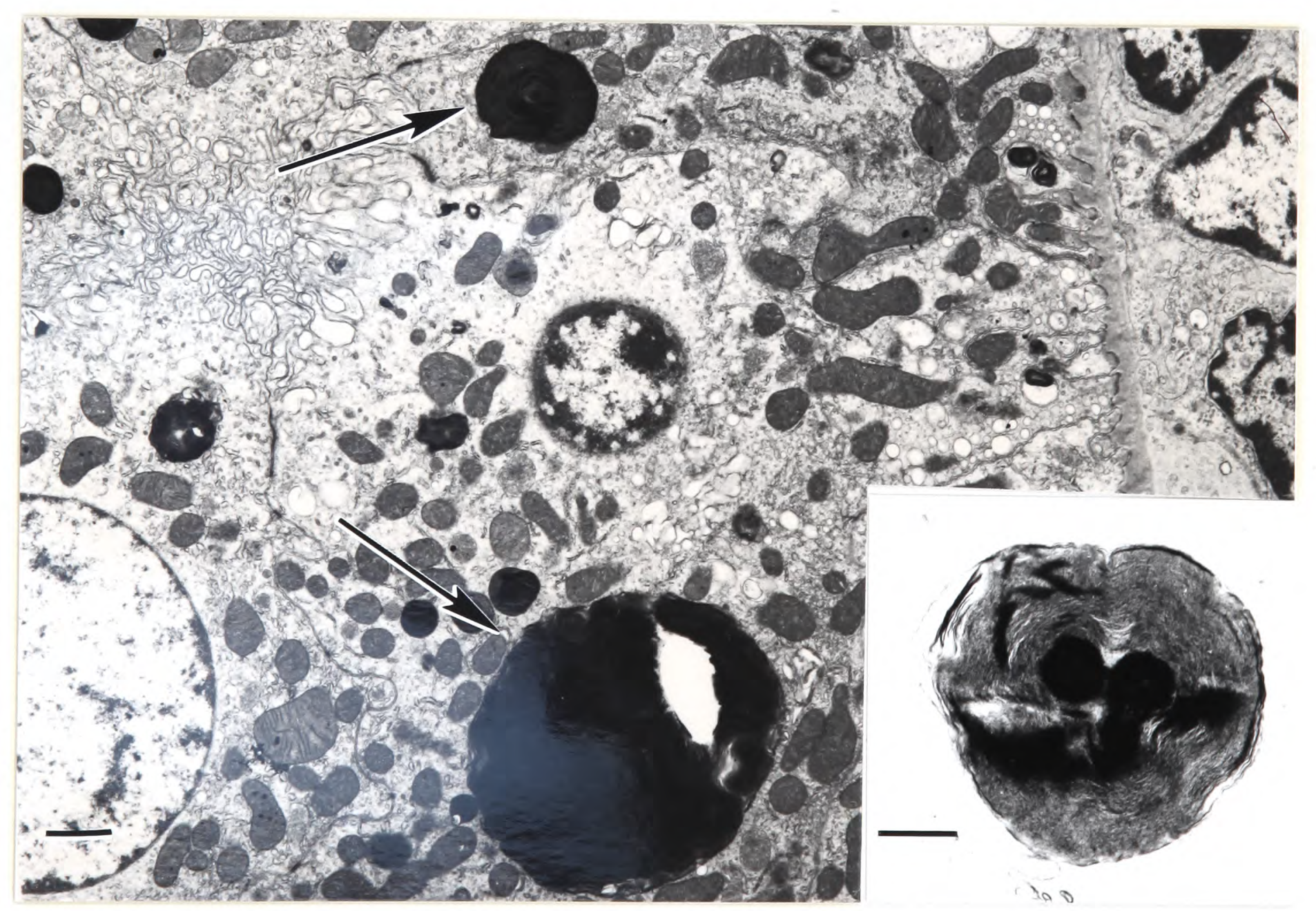

Figure 7.10 - Electron micrograph of a proximal tubular cell from the kidney in Figure 7.9. There are osmophilic inclusions (arrows). Inset is an under-exposed view to show the lamellar structure of one of these bodies. Bars are $1 \mu \mathrm{m}$.

Electron microscopy showed osmophilic deposits in tubular cells in regions of this kidney (Figure 7.10). This remains unexplained. The expanded interstitium included many fibroblast-like cells (Figure 7.11). 

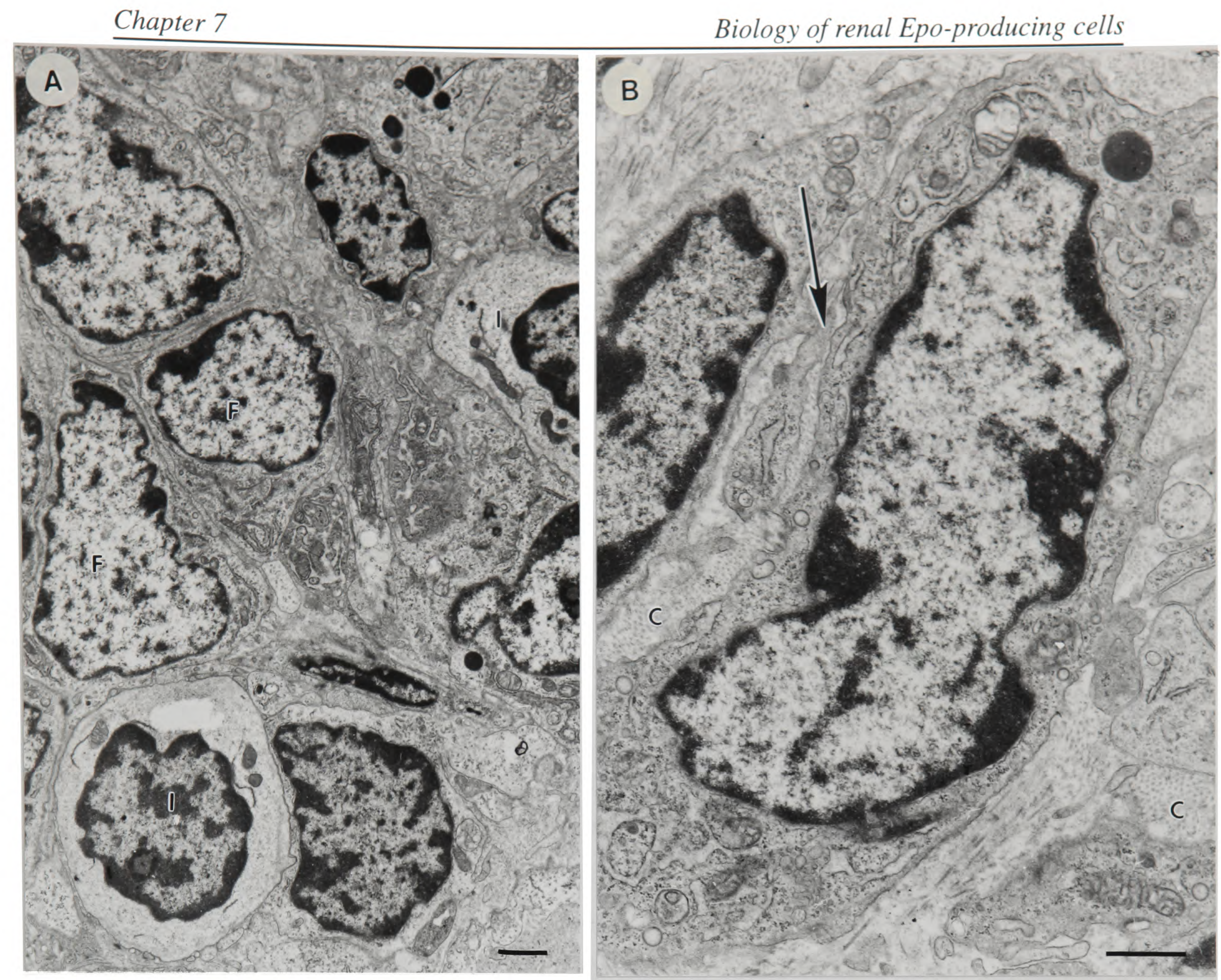

Figure 7.11 - A. Electron micrograph of conventionally fixed material from an interstitial "tumour" found in an Epo-TAg ${ }^{H}$ homozygote, showing inflammatory cells (I) and fibroblastlike cells $(F)$. Bar is $1 \mu \mathrm{m}$.

$B$. Higher magnification view of two fibroblast-like cells showing close apposition of cell processes (arrow) and surrounding bundles of collagen (C). Bar is $1 \mu \mathrm{m}$.

\subsubsection{Foetal expression of Epo-TAg}

SV40 T Ag might be more effective in transforming cells while organs were still developing. Consequently, it would be attractive to study homozygous offspring of homozygous mothers. Although the Epo-TAgH homozygous females were occasionally fertile, they often lost their offspring very shortly after birth. However, one offspring of such a mating survived to the age of 200 days without developing a renal tumour.

\subsubsection{Conclusions}

The observation that the expression of SV40 T Ag can lead to expansion of the renal interstitium, and that mitosis can occur in cells expressing Epo-TAg demonstrates that Epoproducing cells are not terminally differentiated. It appears that the development of renal tumours requires high level expression of Epo-TAg for several months. The mechanisms by which SV40 T Ag transforms cells are not fully understood. This viral oncogene has 
several relevant properties; in particular it binds p53, p107 and the retinoblastoma gene product (Fanning 1992). Since tumours at other sites were a frequent occurrence there is suggestive evidence that the Epo expressing cells of the kidney are relatively resistant to the transforming effects of SV40 T Ag.

Although SV40 T Ag alone can immortalise cells in culture, many of these cells do not have a fully transformed phenotype (Jat and Sharp 1986). It seems likely that a second signal is a requirement for malignant tumour formation in the current transgenic experiments. That this can be necessary in an analagous situation has recently been demonstrated by Hanahan and colleagues in the beta cells of the pancreatic islet, where for development of tumours expression of IGF-II appears to be an important requirement, in addition to SV40 T Ag (Christofori et al. 1994). Interestingly, another recent study demonstrated that transformation of mouse embryonic fibroblasts by SV40 T Ag required the presence of Type 1 IGF-I receptor (Sell et al. 1993).

\subsubsection{Isolation of cells from the kidneys of Epo-TAg mice}

In the absence of frequent renal tumours, experiments were undertaken to isolate cells from the kidneys of Epo-TAg mice without tumours. The observation that long-term anaemia led to an expansion of the Epo-producing cell population made the kidneys of anaemic Epo-TAgH animals attractive for these experiments. Finely chopped kidney was digested with proteinases, filtered and cultured in dishes either at $1 \%$ oxygen or $21 \%$ oxygen. Three separation protocols were used; one optimised for obtaining antigen-presenting "dendritic" cells (Dr J. Austyn), one for obtaining fibroblasts from the kidney (Dr P. Gallimore) and one for obtaining endothelial cells (Dr R. Bicknell). In some experiments the cultures were continuously exposed to hypoxia following digestion in an attempt to induce Epo-TAg expression. Although cells were obtained in primary cultures by all three methods, these cultures did not contain SV40 T Ag on immunostaining, nor did they contain Epo or EpoTAg mRNA. Following a period of crisis apparently immortalised cells were obtained; however this is not necessarily due to SV40 $\mathrm{T} \mathrm{Ag}$ expression since spontaneous immortalisation does occur in murine cultures. One immortalised, clonal cell line (TG-L) of fibroblastoid morphology was studied in detail. Following hypoxic exposure it did not label for SV40 T Ag or 5'NT on immunostaining, nor did it contain Epo-TAg mRNA or Epo mRNA. This cell line was also negative for the fibroblast marker ER-TR7 and for LCA, desmin, and cytokeratin. It was, therefore, not possible to determine its origin on the basis of immunostaining. Several explanations were considered for these negative results. Firstly, 
it was possible that these cells were the correct cells but had either lost the capacity for Epo expression while proliferating or were missing some other requirement (such as contact with a functioning epithelial cell or a chemical factor present in the renal interstitium). Secondly, the correct cells may have been lost in the disaggregation process.

\subsubsection{Co-culture experiments}

It was possible that cell lines derived from Epo-TAg mice would express the Epo gene under certain culture conditions, but not others. Co-culture experiments were therefore undertaken with functional renal epithelial cells. These experiments were performed in collaboration with Dr Muller and Professor Bauer (Zurich). Cultures were established with proximal tubular cells (PKSV-PR or PKSV-PCT) on the opposite side of a nucleopore membrane from TG-L cells (a clonal fibroblastoid lone from an Epo-TAg mouse). These were then exposed to hypoxia. RNA was extracted and assayed by RT-PCR for Epo mRNA. No Epo-mRNA was detected.

\subsubsection{Cell-separation experiments}

To test the possibility that Epo-producing cells were lost during digestion of the kidney, experiments were performed in which cells from the disaggregated kidneys of acutely anaemic Epo-TAg ${ }^{\mathrm{H}}$ mice were examined. SV40 T Ag would serve as a marker to identify the cells during this procedure. Experiments in mice (data not shown) demonstrated that after hypoxic exposure SV40 T Ag persisted in interstitial cells for at least 72 hours after return to normoxia.

Kidneys were digested, the suspension sieved and separated into fractions by centrifugation on Percoll gradients. Cytospin preparations of these fractions were not straightforward to interpret, due to high levels of non-specific antibody binding. In only one experiment was SV40 T Ag clearly demonstrable in any cells. In this experiment the kidney of an iron deficient Epo-TAgH mouse was digested; SV40 T Ag positive nuclei were found adherent to the outside of tubular fragments (Figure 7.12). These nuclei were surrouded by very little cytoplasm and may not have been viable. This preparation was placed on culture dishes. The following morning cytospins from both the culture medium and the adherent cells were made. Neither contained nuclei positive for SV40 T Ag. 


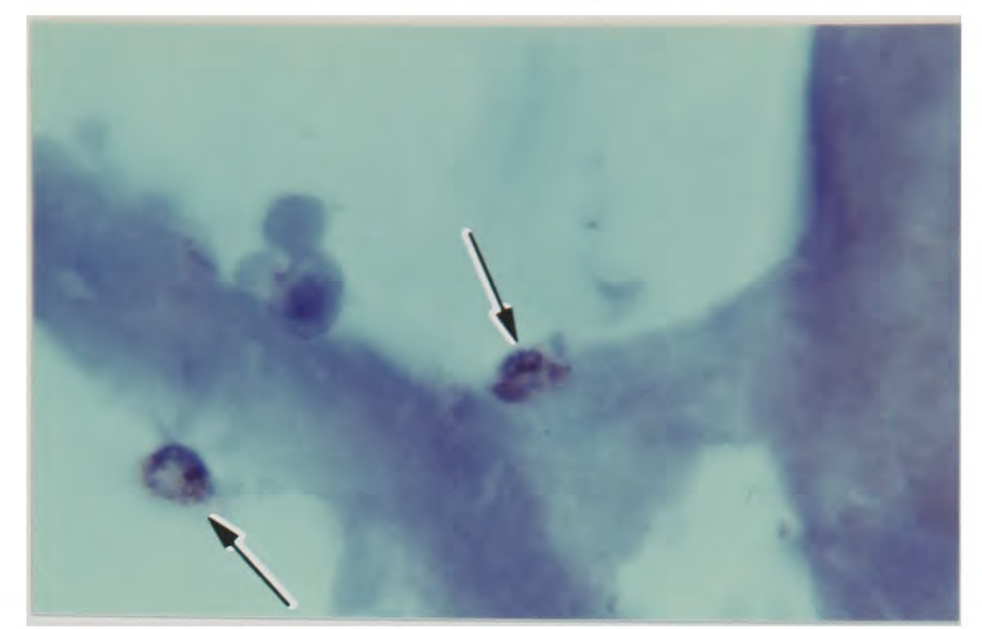

Figure 7.12 - Cytospin preparation from an anaemic Epo-TAg ${ }^{H}$ mouse labelled for SV4O T Ag. Positive nuclei (arrows) are seen adhering to the outside of tubular remnants, but they appear to have very little cytoplasm. Magnification $x 500$.

Since SV40 T Ag appears to be stable in the renal interstitial cells for at least 72 hours in intact mouse kidney, this makes it likely, but does not prove, that the cells had been lost in the disaggregation process. The nuclei adherent to the tubules were probably non-viable, since they could not be recovered the following morning. The origin of the fibroblastoid cells obtained in these cultures, and their relationship to the Type 1 interstitial cells therefore remains uncertain.

\subsubsection{Immunoprecipitation of SV40 TAg}

Staining of cytospins from digested kidney was unsatisfactory due to a high level of nonspecific labelling. Protein extraction followed by immunoprecipitation and Western blotting was considered as an alternative method of detection of SV40 T Ag. Although SV40 T Ag was readily detected in $500 \mu \mathrm{g}$ of protein extracted from Cos 7 cells, it could not be detected in the same amount of protein extracted from the kidney of an acutely anaemic Epo-TAg ${ }^{H}$ mouse (Figure 7.13).

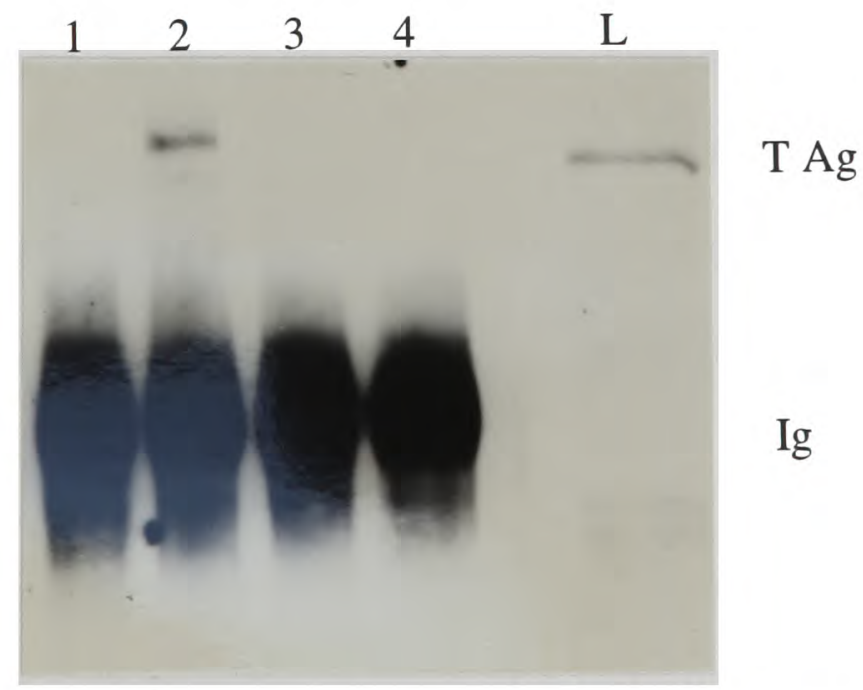

Figure 7.13 - Detection of SV4OTAg by immunoprecipitation (pAb419 and Protein G sephadex) followed by Western transfer and detection with pAb423. Lanes 1 to 4 loaded with immunoprecipitates; 1 non-transgenic kidney, 2 Cos 7 cells, 3 \& 4 kidneys from anaemic EpoTAg mice. Lane L was loaded with Cos7 lysate (without prior immunoprecipitation). SV40 T Ag is identified in the Cos 7 cells, but not the kidney specimens. In the immunoprecipitates there is a dense band due to immunoglobulins. 


\subsubsection{Alternative methods of obtaining cells from the kidney}

A different procedure, widely used to obtain fibroblasts from skin and previously used to grow cells from the kidney (Davis et al. 1983) is now being investigated. This involves cutting small pieces of kidney and placing them on a culture dish in a small amount of medium, and allowing cells to grow out of the fragments. Since this procedure does not require digestion of the kidney, it might allow fragile cells to grow out. Again, primary cultures were established frequently, but they did not contain Epo-TAg mRNA or SV40 T Ag. Interestingly when explants were observed in parallel from an Epo-TAgH homozygote, an Epo-TAgH heterozygote and non-transgenic littermates no striking differences were observed.

\subsubsection{Conclusions}

Nuclear SV40 T Ag in the Type 1 interstitial cells should provide a stable marker allowing the identification of these cells during isolation procedures. The current evidence would suggest that the Type 1 interstitial cells can easily be lost at an early stage during the procedures attempted. Although morphologically "fibroblastoid" cells were obtained frequently they did not express the Epo-TAg or native Epo genes.

These observations raise the possibility that "fibroblasts" cultured from the kidney originate from a cell population other than that which produces Epo. Alternatively, it is possible that the appropriate cells are present in these cultures, but have lost the ability to produce Epo. This could be due to "de-differentiation" of the cells themselves or to the lack of an essential requirement for Epo production in tissue culture.

\subsubsection{Investigation of conditionally immortalised fibroblast cell lines}

Following the identification of the Epo producing cells as fibroblastoid cells in the renal interstitium, it was logical to screen recognised fibroblasts of renal origin for Epo production. For clarity some of the resultant negative data (concerning Cos 7 cells and NRK-49F cells) is presented in more detail in Chapter 3, since it is relevant to the issue of hypoxic sensing by cells which do not produce Epo.

An effective system for immortalising cells and retaining a differentiated phenotype has been to explant cells from a transgenic mouse line which expresses a temperature sensitive mutant form of SV40 T Ag under the control of the mouse MHC H-2 $\mathrm{K}^{\mathrm{b}}$ promoter (Jat et al. 1991). At $37^{\circ} \mathrm{C}$ the product is unstable and the effects on the mouse are minimal. Cell lines derived from these mice can be grown in tissue culture at $33^{\circ} \mathrm{C}$ with $\gamma$-interferon ( 10 units/ 
$\mathrm{ml}$ recombinant mouse IFN $\gamma$; Genzyme, West Malling). SV40 T Ag production is stimulated, the oncogene is stable and the cells proliferate. When the temperature is raised to $37^{\circ} \mathrm{C}$ the SV40 T Ag is unstable, and the cells become quiescent.

Six conditionally immortalised cell lines were assayed for Epo gene expression. These lines were generously provided by Dr A. Woolf (Woolf et al. 1993). Five lines were derived from adult kidneys (termed Fib 5, Fib 6, Fib 7, Fib 10, Fib 12). One (M5) was derived from a foetal kidney, and has been shown to express hepatocyte growth factor/scatter factor. This was of particular interest since this factor is known to be secreted by Ito cells in the liver, and they cease to express the gene in tissue culture as they become more myofibroblastoid (Schirmacher et al. 1992). Given the similarities between the Type 1 interstial cells in the kidney and the Ito cells, this suggested that the M5 cell line might also express Epo.

Cells were grown under permissive conditions, and then incubated for 7 days at $37^{\circ} \mathrm{C}$ to allow the SV40 T Ag to decay. They were then exposed to hypoxia for 16 hours. Total mRNA was extracted, hybridised to the mouse Epo probe and analysed by RNase protection. No Epo transcripts were detected.

\subsubsection{Conclusions}

One possible explanation for this negative result is that these cells, which were selected from primary cultures on the basis of a fibroblastoid morphology, are not derived from the Type 1 interstitial fibroblasts. If these cells are representative of renal interstitial fibroblasts then this would suggest that some aspect (or aspects) of the renal interstitium in vivo, which is not present in tissue culture, is necessary for Epo expression. Alternatively the process of conditional immortalisation followed by decay of SV40 T Ag may have altered the phenotype in a way that precludes Epo gene expression.

\subsubsection{Response of the renal interstium to renal injury}

The impairment of Epo expression in renal disease is striking and the reasons for it are unknown. In order to investigate this issue, renal injury was combined with visualisation of Epo-TAg expression at a cellular level. A second reason for these experiments was that renal injury might provide a second stimulus facilitating transformation of the interstitial cells by SV40 T Ag. 
Epo-TAgH mice, in which Epo-TAg expression can be visualised and combined with other markers, offer a valuable experimental system for investigating this failure of renal Epo expression. It is, however, appropriate to consider some difficulties with this approach. Firstly, in order to demonstrate Epo-TAg expression in heterozygous Epo-TAgH mice it is necessary to render the animals hypoxic or anaemic; this might modify the renal injury. Alternatively, homozygous Epo-TAgH animals could be studied where the anaemia would act as a stimulus to continued Epo-TAg expression. A concern with this second approach is the relatively long half-life of SV40 T Ag in the interstitial cells; thus for some time (at least 72 hours) SV40 T Ag would be expected to persist even if de novo formation had ceased. Moreover, the interstitium has an increased number of cells in the homozygotes. Since the problems with the two approaches (using homozygous and heterozygous mice) are different both were adopted. In the two models of renal injury which were global (ureter ligation and clamping the renal pedicle) mRNA was analysed as a further means of assessing the level of gene expression.

Three standard models of renal injury were investigated; ligation of the ureter (Nagle et al. 1973), clamping the renal pedicle (Arendshorst et al. 1975) and needlestick injury (Argyris and Trimble 1964). In each experiment the left kidney was injured and the contralateral kidney served as a control. In this context, it is important to appreciate that there is information that the uninjured kidney is affected by injury to the contralateral organ; comparatively, however, these changes are relatively minor (Wesson 1989). The animals were sacrificed at 6-8 days following injury. Heterozygous animals were exposed to an apparent altitude of 7,200 $\mathrm{m}$ for 72 hours prior to sacrifice. The kidneys of some animals were perfusion-fixed for optimal morphological assessment. Kidneys from the other animals were each divided for mRNA analysis and for cryosectioning. The latter allowed identification of SV40 T Ag in sections that were acetone-fixed for optimal antigen preservation, although the morphological preservation was less good.

\subsubsection{Ureter ligation}

One week following ureter ligation the kidney was hydronephrotic, with a dilated pelvis and thinning of the cortex. Microscopically, the tubules were dilated. In the interstitium the number of nuclei appeared to be increased and there was a degree of fibrosis. These observations were similar in transgenic and non-transgenic animals. Labelling for SV40 T Ag in the damaged kidney demonstrated positive cells in the cortex, but many fewer than in the control kidney (Figure 7.14A,B). Quantitative assessments of the number of cells 
containing SV40 T Ag are given in Table 7.9. Positive cells in the damaged kidney tended to be in the deeper cortex and outer medulla. Similar results were obtained in the homozygous and heterozygous animals.

\begin{tabular}{|c|c|c|c|}
\hline Animal & Region & Injured (left) & Control (right) \\
\hline Heterozygote & Outer cortex & $0.5+/-0.8$ & $13.4+/-6.3$ \\
& Inner cortex/outer stripe & $9.3+/-4.5$ & $28.0+/-5.2$ \\
\hline Heterozygote & Outer cortex & $0.0+/-0.0$ & $5.9+/-6.0$ \\
& Inner cortex/outer stripe & $0.8+/-1.0$ & $15.4+/-8.3$ \\
\hline Homozygote & Outer cortex & $1.0+/-1.4$ & $57.3+/-24.0$ \\
& Inner cortex/outer stripe & $73.5+/-20.7$ & $101.0+/-30.5$ \\
\hline Homozygote & Outer cortex & $19.8+/-12.6$ & $44.8+/-16.4$ \\
& Inner cortex/outer stripe & $33.0+/-16.4$ & $76.8+/-25.7$ \\
\hline
\end{tabular}

Table 7.9 - The effects of ureter ligation and consequent hydronephrosis on the capacity for Epo gene expression. Heterozygous animals were exposed to hypobaric hypoxia for 72 hours. In homozygous animals the constitutive anaemia was sufficient to stimulate Epo-TAg expression. Animals were sacrificed 6 to 8 days following the surgical procedure. The number of positive nuclei (mean \pm s.d., 4 to 6 observations) per unit area $\left(0.25 \mathrm{~mm}^{2}\right)$ is given. In the injured kidney in each case there were substantially fewer cells expressing Epo-TAg; these were located predominantly in the deep cortex and outer stripe of the outer medulla.
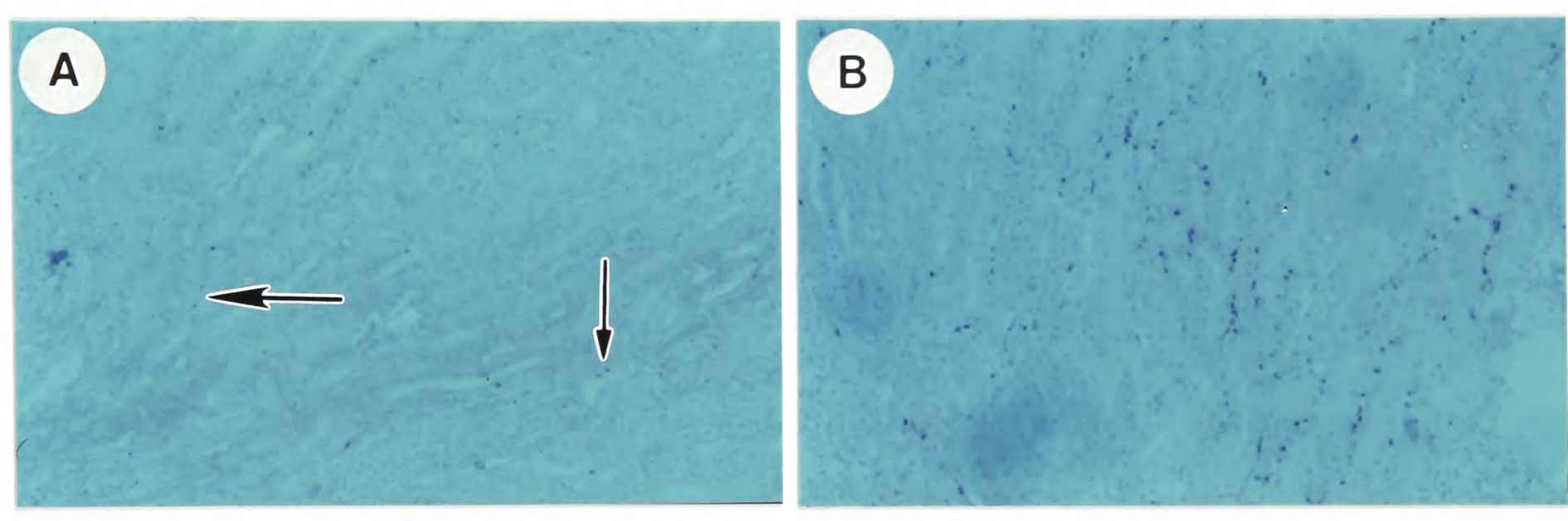

Figure 7.14 - The effect of ureter ligation on the expression of the Epo-TAg' gene in a homozygous animal. Labelling for SV4O T Ag in (A) the obstructed left kidney and (B) the control (right) kidney from the same animal. Both micrographs extend from the outer medulla (bottom left) towards the superficial cortex (top right). Positive nuclei are much more frequent in the control kidney. In the obstructed kidney positive cells (arrows) still appear to be located predominantly in the deeper part of the cortex. Magnification $x 75$. 


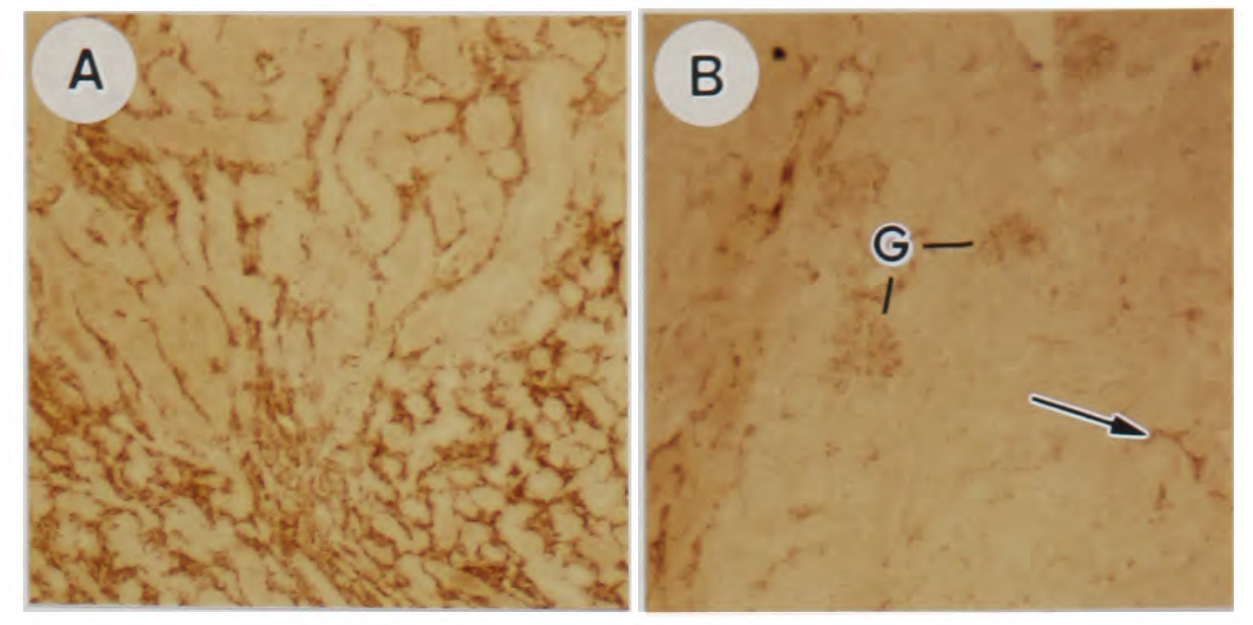

Figure 7.15 - The effect of ureter ligation on interstitial expression of desmin. (A) shows the obstructed (left) kidney and $(B)$ shows the control (right) kidney from a heterozygous animal. Desmin labelling (brown) is more marked in the interstitium of the obstructed kidney. In the control kidney there is some labelling in the interstitium (arrow) and the glomeruli (G). Both micrographs extend from outer medulla (bottom) into the cortex (upper part). Magnification $x 75$.

Analysis of mRNA by RNase protection assay in the homozygous animals confirmed expression of the Epo-TAg gene, but at a reduced level compared to the contralateral kidney, both at 1 and 7 days after the injury. When heterozygous animals were analysed (following 72 hours of hypoxic exposure) the levels of Epo-TAg mRNA were very low; presumably reflecting adaptation to the hypoxic stimulus.

The expression of other Type 1 interstitial cell-associated antigens in the interstitium was also examined. There was a very substantial increase in desmin expression (Figure 7.15). Labelling with ER-TR7, and 5'NT expression was decreased in intensity. Interestingly, 5'NT expression appeared relatively preserved in the deep interstitium, in the region where Epo-TAg was expressed. LCA positive cells were very substantially increased in number. When sections were double-labelled for SV40 T Ag and LCA, the cell populations were clearly distinct. The LCA positive cells were predominantly in the outer cortex and were often grouped together. There appeared to be few SV40 T Ag positive nuclei in the regions where LCA positive cells were aggregated together.

Aspects of these observations in mice are consistent with previous studies in other species. In rabbits, obstruction of the ureter leads to proliferation of interstitial fibroblasts and an increase in contractility and smooth muscle actin expression (Nagle et al. 1973; Nagle et al. 1975). At an ultrastructural level, cells in the interstitium of the obstructed kidney were shown to contain filaments characteristic of myofibroblasts (Nagle et al. 1975). An increase in the number of leucocytes has been described previously in the obstructed rat kidney; the increase being largely due to a fifteen-fold increase in the number of macrophages (Schreiner et al. 1988). How ureteric obstruction leads to these changes remains unknown. 


\subsubsection{Clamping the renal pedicle}

Following an ischaemic insult to the kidney, acute tubular necrosis occurs with renal failure. Clamping the renal atery in the rat has been used to investigate the mechanisms of acute renal failure (e.g. Arendshorst et al. 1975) and subsequent tubular regeneration. In the current experiments the renal pedicle was clamped for 45 minutes or 60 minutes. One week later, following perfusion-fixation, many tubules appeared relatively normal; in other tubules the epithelium was flattened. The interstitium was expanded, but to a lesser degree than was seen following ureter ligation. Labelling for SV40 T Ag demonstrated fewer positive cells in the injured kidney than the control kidney. The numbers of positive cells are given in Table 7.10. No tubular cells were positive for SV40 T Ag. It was not possible to correlate tubular morphology in a particular region with T Ag expression. When mRNA was analysed 7 days following the injury there was expression in both kidneys, with the injured kidney showing an approximately two-fold reduction compared to the control kidney.

\begin{tabular}{|c|c|c|c|}
\hline Animal & Region & Injured (left) & Control (right) \\
\hline Heterozygote & Outer cortex & $2.6+/-2.7$ & $14.3+/-6.3$ \\
& Inner cortex/outer stripe & $11.3+/-10.3$ & $55.3+/-7.7$ \\
\hline Heterozygote & Outer cortex & $1.5+/-2.6$ & $0.9+/-1.8$ \\
& Inner cortex/outer stripe & $1.9+/-3.8$ & $18.0+/-15.5$ \\
\hline Homozygote & Outer cortex & $55.8+/-19.8$ & $226.0+/-45.3$ \\
& Inner cortex/outer stripe & $84.5+/-17.6$ & $260.3+/-21.5$ \\
\hline
\end{tabular}

Table 7.10 - The effects of ischaemic injury (clamping the renal pedicle) on the capacity for Epo gene expression in homozygous and heterozygous Epo-TA $\mathrm{g}^{H}$ mice. The latter were exposed to hypobaric hypoxia for 72 hours prior to sacrifice, in order to induce Epo-TAg expression. Animals were sacrificed 6 to 8 days following the surgical procedure. The number of positive nuclei (mean \pm s.d., 4 to 6 observations) per unit area $\left(0.25 \mathrm{~mm}^{2}\right)$ is given. In the injured kidney in each case there were substantially fewer cells expressing Epo-TAg; these were located predominantly in the deep cortex and outer stripe of the outer medulla.

The expression of other antigens was also examined in these animals. Desmin expression was increased (especially in the outer stripe of the outer medulla), and there was some expression of smooth muscle actin. Expression of ER-TR7 was widespread in the interstitium, and covered a greater area than in the contralateral kidney, but was decreased in intensity. Expression of 5'NT was reduced. In the injured kidney there was an excess of cells bearing LCA.

\subsubsection{Needlestick injury}

Passing a needle through the renal cortex has been studied as a means of producing a focal injury (Argyris and Trimble 1964). One week after passing a 23 gauge needle through the left kidney in six positions, focal areas of injury were clearly identifiable. These were 
wedge-shaped, extending from the cortex to the inner stripe of the outer medulla. There was expansion of the interstitium, with increased numbers of interstitial nuclei and evidence of fibrosis and tubular damage.

Sections were cut in a plane orthogonal to the direction of the needle pass and labelled for SV40 T Ag. The number of cells containing SV40 T Ag in the injured regions was greatly reduced. However, occasional cells were positive for SV40 T Ag, even when adjacent epithelial cells appeared severely damaged. In the regions of injury there were more interstitial nuclei and labelling of 5'NT was less intense. Desmin expression was increased and weak smooth muscle actin labelling was observed. ER-TR7 labelled a larger area in the injured regions, but at a lower intensity. There was also an excess of cells bearing LCA.

\subsubsection{Conclusions.}

Six to eight days following renal injury there are major effects on induced renal expression of Epo-TAg. These effects are clear both in a globally injured kidney and in a focally injured region. They were seen in the context of three different types of injury. The effect was similar whether capacity for Epo-TAg expression was assessed by response to chronic anaemia (homozygotes) or acute hypoxia (heterozygotes).

A reduced, but significant, level of Epo expression in the damaged kidney is consistent with clinical observations in renal disease and previous experiments on laboratory animals. Jacobson observed that bilateral ureteric obstruction reduced, but did not abolish the erythropoietic response to cobalt (Jacobson et al. 1957). Since the reduction was much less than that of bilateral nephrectomy the residual erythropoietin response was likely to originate from the obstructed kidneys. Recently Necas has shown a reduced (by about $70 \%$ ) but detectable renal Epo mRNA response to carbon monoxide in unilaterally obstructed mouse kidneys compared to the contralateral control kidney (Necas 1994). In rats with uraemia following subtotal nephrectomy the remnant kidney expresses Epo mRNA at reduced levels but responds to hypoxia with a 50 fold induction of Epo expression (Tan et al. 1991).

In the current studies it was possible to localise Epo-TAg expression at a cellular level in the injured kidney and to correlate it with changes in the expression of other antigens. Where Epo-TAg expression was deficient (either globally or focally) increased expression of desmin, and (to a lesser extent) alpha smooth muscle actin was observed. In these areas there was reduced expression of $5^{\prime} \mathrm{NT}$. The changes are summarised in Table 7.11. In all three models of renal injury the changes in the interstitium were similar. 


\begin{tabular}{|l|c|c|c|c|c|c|}
\hline & SV40 TAg & 5'NT & desmin & s.m.actin & ERTR7 & LCA \\
\hline Ligation & $\downarrow$ & $\downarrow$ & $\uparrow$ & $\uparrow$ (weak) & $\downarrow$ (expanded) & $\uparrow$ \\
\hline Needlestick & $\downarrow$ & $\downarrow$ & $\uparrow$ & $\uparrow$ (weak) & $\downarrow$ (expanded) & $\uparrow$ \\
\hline Clamp & $\downarrow$ & $\downarrow$ & $\uparrow$ & $\uparrow$ (weak) & $\downarrow$ (expanded) & $\uparrow$ \\
\hline
\end{tabular}

Table 7.11 - Effects of injury on expression of antigens in the renal interstitium. Three different stimuli were assessed, both in homozygous and in heterozygous Epo-TAg ${ }^{H}$ mice. The latter were subsequently exposed to 7,200 $\mathrm{m}$ for 72 hours to induce Epo-TAg expression. In all cases the volume of the interstitium and the number of nuclei appeared to be increased.

This study cannot directly address whether the cells responsible for Epo expression are present in the regions where Epo-TAg expression was not observed. However, it seems unlikely that these cells have disappeared from these regions, and more likely that they are present and expressing increased levels of desmin. When an obstructed kidney was labelled for both SV40 T Ag and desmin it was clear that some of the cells expressing high levels of desmin were labelled for SV40 T Ag (data not illustrated). This demonstrates that at least a proportion of the cells expressing high levels of desmin retain the capacity to express the Epo-TAg gene.

The reason for reduced Epo expression in an injured kidney remains unclear. However, when the kidney is injured it appears that there is predominantly a reduction in the number of cells expressing Epo, rather than the level of expression per cell. Broadly, the level of Epo-TAg mRNA was proportional to the number of cells labelling for SV40 T Ag, both in injured and control kidneys (data not shown). This suggests that the level of expression per cell remains unaltered following injury. The distribution of the cells expressing Epo-TAg also appeared similar to that seen in uninjured kidneys, with positive cells located in the deep cortex. Although the number of cells expressing the Epo gene is reduced, the total number of Type 1 interstitial cells is probably increased in response to injury.

Why then is expression of the Epo gene reduced? A number of possible explanations could be proposed. Firstly it is possible that changes in blood flow are critical. Marked changes in blood flow occur following ligation of the ureter and during recovery from renal ischaemia. Secondly, local oxygen consumption (e.g. by functioning tubular epithelium) may be important. Thirdly, it is possible that leucocytes (or a subset of these cells) are modulating Epo expression and the changes in this population in response to injury are impressive. Finally, it is possible that the Type 1 interstial cells themselves switch to an injury-repairing phenotype, which involves suppression of the capacity for Epo gene expression. 
Considerable interest has focused on the behaviour of renal fibroblasts in culture (e.g. Rodemann et al. 1991). Several studies suggest that fibroblasts cultured from abnormal kidneys behave differently from fibroblasts derived from control specimens (Davis et al. 1983; Rodemann and Müller 1990 \& 1991). How these cultured fibroblasts relate to the Epo-producing cells in the intact kidney is uncertain. Epo-TAg ${ }^{\mathrm{H}}$ mice may be useful in addressing some of the outstanding issues. For instance, the fate of the Epo-producing cells could be followed over a period of a few days, since SV40 T Ag appears to be stable for this period.

A final point to note is that in these experiments no evidence for Epo-TAg expression in other cell types (such as leucocytes or epithelial cells) was observed. Thus both in the normal, and in the injured kidney Epo-TAg (and presumably Epo) gene expression appears to be restricted to the Type 1 interstial cells.

\subsection{GENERAL DISCUSSION}

The principal result in this chapter is that expression of SV40 T Ag at a high level in the renal interstitium leads to proliferation of the interstitial cells. Furthermore, this can lead to the development of tumours. Tumours were only produced in the context of chronic anaemia and a homologous recombination, resulting in a sustained high level of Epo-TAg expression in the renal interstitium. This was somewhat surprising, given the ease with which endocrine tumours of the pancreas were observed in an analagous experiment (Hanahan et al. 1995). One previous study is of interest in this context. In this study, SV40 T Ag was expressed under the control of a Class $1 \mathrm{MHC}$ promoter, which resulted in a low level of expression in all tissues and a higher level of expression in lymphoid tissues (Reynolds et al. 1988). In spite of this distribution of expression, endocrine tumours were much more common than at any other site, suggesting that these cells are more susceptible to the effects of SV40 T Ag. Data presented in this chapter suggests that renal Type 1 interstitial cells are relatively resistant to the effects of SV40 $\mathrm{T} \mathrm{Ag}$.

Cell cultures with fibroblastoid characteristics (from Epo-TAg mice or other sources) did not express the Epo gene. This may be because the cells are fragile and easily destroyed during disaggregation procedures; although this was addressed, the results were not entirely conclusive. Several different protocols failed to obtain Epo-TAg expressing lines in spite of the observation of many cells in the cultures with fibroblastoid morphology. Furthermore, renally derived "fibroblasts" from other sources did not express Epo in response to hypoxia. 
In response to injury the interstitium undergoes changes, involving greatly reduced Epo gene expression, decreased 5'NT expression, and increased desmin expression. One possibility is that this is due to a phenotypic change in the Type 1 interstitial cells, with a switch from a "quiescent" Epo-expressing phenotype to an "activated" phenotype in response to injury. Interestingly, there is substantial evidence for such a phenotypic change in the Ito cells of the liver from quiescent fat-storing cells to myofibroblasts in response to injury or explantation (Ramadori 1991).

It remains possible that Epo-producing cells are lost when the kidney is disaggregated. Equally, it may be that once these cells are explanted they are outgrown by other species under tissue culture conditions. Finally, it is possible that cultured renal fibroblasts are derived from Epo-producing cells but a phenotypic or environmental change has resulted in loss of the capacity for Epo gene expression. 


\section{Homologous recombination at the Epo locus}

\subsection{INTRODUCTION}

In the course of generating Epo-TAg transgenic mice homologous recombination (HR) occurred between the microinjected Epo-TAg DNA and the Epo locus (see Chapter 4). This event resulted in the integration of the $2.7 \mathrm{~kb}$ of viral sequence in the 5 ' UTR of the native Epo gene. This targeted modification of the Epo locus was fortuitous, and provided a fusion gene in which the viral marker was flanked by all cis-acting sequence at the Epo locus. Furthermore, the insertion of the viral sequence, which included polyadenylation signals, disabled the Epo gene, with the result that homozygotes had a severe anaemia.

It is well-established that mammalian systems have the ability to homologously recombine DNA substrates (Bollag et al. 1989). This is an efficient process, both between extrachromosonal DNA molecules and between different sites in chromosonal DNA. However, whereas in lower organisms (bacteria, yeast and trypanosomes) if homologous DNA is introduced into the cell HR is the rule, in mammalian cells it is the exception. Thus in mammalian cells introduced DNA is generally integrated at heterologous sites; HR occurs at a much lower frequency.

In recent years, it has proved possible to create targeted mutations in the mammalian genome. Null mutations have been produced in a range of genes and the phenotypic effects have yielded much useful information (Capecchi 1989). Many mouse loci have now been succesfully targeted. The commonly used technique is to electroporate totipotential stem cells with a vector which contains homology with the target sequence and a resistance marker. The cells are placed under selective pressure to exclude those which have not expressed the resistance marker. Clones of cells are then tested for the desired event by PCR. The rate of HR to heterologous insertion can be enhanced by several strategies. Thus a marker which will usually be transcriptionally deficient if integrated at a random site can be used. Another strategy uses negative selection, by placing a second marker at the end of the homologous sequences (Mansour et al. 1988). This non-homologous sequence on the end of the incoming DNA is removed when the DNA is integrated in a targeted fashion.

The established technique using stem cells has several disadvantages. Firstly, the establishment of totipotential stem cell lines is not straightforward, and restricts the genetic background and species available for targeted mutation. Secondly, maintaining these cell lines in a totipotential form while they are being genetically modified is a demanding process. 
Thirdly, because the rate of DNA integration is low (of the order of 1 event per 10,000 electroporated cells), the technique requires the use of a selectable marker; this in turn requires a considerable length of coding sequence in the targeting construct. An exception to this is when the targeted gene itself can be used as a selectable marker, as in the case of hypoxanthine-guanine phosphoribosyl transferase (Doetschmann et al. 1987). However, at other loci if a subtle mutation is desired, a two-stage procedure is likely to be necessary. Such systems have been devised; "in and out" (Valancius and Smithies, 1991), "hit and run" (Hasty et al. 1991) or more recently based on the bacteriophage P1 Cre-loxP system (Baringa 1994). Thus a system allowing targeted modification at a high rate by direct zygote injection would be potentially attractive. The single observation of such a recombination in the Epo- $\mathrm{TAgH}^{\mathrm{H}}$ line (one of only thirteen transgenic lines created) raised the possibility that this would be a feasible system for engineering such mutations. While this may have been the chance observation of a rare event, it is also possible that the frequency of such events would be high enough to make such a system workable.

In order for zygote injection to be a workable means of modifying the mouse genome, there are essentially three requirements. Firstly, the frequency of obtaining homologous recombinant mice would need to be of the order of 1 per 1000 zygote injections performed, or greater. Secondly, a secure and sensitive system for detection of HR would be necessary. Finally, homologous recombinant events would have to be faithful, giving rise only to the desired mutation.

The possibility that direct injection of mouse zygotes would fulfil these requirements has been examined once previously. The experimental approach used was to attempt to correct a deletion at the $\mathrm{E} \alpha$ locus (Brinster et al. 1989). 10,602 zygotes were injected; 1,841 pups were born of which 506 had integrated the transgene. In one of these the integration had occurred in a targeted fashion. Not only was the targeting efficiency low, but in the single homologous recombinant event sequence analysis showed that the recombined locus differed at individual bases from both the incoming and recipient DNA.

However, subsequent experiments in stem cells have defined two parameters which influence the rate of homologous integration, and which were sub-optimal in Brinster et al's study. These factors are the length of homology and the degree of isogenicity of the incoming and target DNA. It is likely that these parameters will also influence the targeting frequency 
(that is the rate of homologous compared to heterologous events) in zygotes as well as in totipotential stem cells. Both of these parameters were relatively more favourable in the Epo-TAg experiment.

The influence of the length of homology in the targeting vector in stem cell experiments has been examined by comparing the targeting frequency of vectors containing different lengths of homology at a single locus. At one locus an increase from $2 \mathrm{~kb}$ to $14.6 \mathrm{~kb}$ resulted in an increase in the frequency of targeted events per million electroporated cells by two orders of magnitude (Deng and Capecchi 1992). Furthermore, it is quite likely that this experiment underestimated the effect on the rate of targeted events, since electroporation becomes progressively less efficient at introducing DNA into the cells as the size of DNA molecule is increased.

The degree of homology between the incoming and target DNA has been shown to be important, with a variable but significant (2 to 10 fold) increase in targeting frequency when isogenic DNA is used (Te Riele et al. 1992; Deng and Capecchi 1992). Differences between the target and incoming DNA are also likely to be important in determining the fidelity of recombination events. It is probable that base differences trigger a mismatch repair mechanism at the time of recombination, which then leads to unpredictable products.

Compared to Brinster et al.'s study, the Epo-TAg construct had more homologous sequence (total $16.5 \mathrm{~kb}$ ). Whether the sequence was isogenic is clearly an important question. Since the recipients were CFLP $\times F_{1}(C 57 / B L 10 \times C B A)$, and the incoming DNA was derived from a commercial Balb/c library (Stratagene), this seemed unlikely. However, on enquiry, a previous investigator had found two ferritin alleles in this library; one was compatible with a Balb/c origin, but the other was not. Of those strains examined, this second ferritin allele would have been compatible with a CBA, DBA/2 or C57/BL6 origin (Yachou et al. 1991). It was therefore possible that the injected DNA and the recipient allele were in fact isogenic. This would be in contrast to Brinster et al.'s previous study in which an Eod allele was used to target E $\alpha$ s or E $\alpha b$ alleles (Brinster et al. 1989).

Thus those factors which are known to influence the frequency of gene targeting in stem cells were probably favourable in the case of the Epo-TAg construct. Other factors, some of which are likely to be target-specific, are likely to influence the rate of HR. For instance, Song and colleagues observed a ten-fold variation in the incidence of HR with the same 
sequence integrated at different sites in a somatic cell line (Song et al. 1987). At any rate, the observation of recombination in the Epo-TAgH mice at least shows that HR can occur at the Epo locus.

Different experiments have indicated a wide variation in estimated frequency of HR versus heterologous insertion. Interpretration of some of these studies is difficult since the rate of heterologous integration is estimated from the number of colonies expressing a resistance marker, which may not be adequately expressed at all sites within the genome. This introduces positive selection for HR. However, at least three studies in which there was no selection for HR suggest that a targeting rate of 1 homologous event per 100 integrations is achievable (Table 8.1)

\begin{tabular}{|l|l|l|l|}
\hline \multicolumn{1}{|c|}{ Authors } & \multicolumn{1}{c|}{ Targeting frequency } & \multicolumn{1}{c|}{ Cell type } & Introduction method \\
\hline Song et al. 1987 & $1: 76$ to $1: 500$ & Somatic & Ca phosphate \\
\hline Zimmer et al. 1989 & $1: 30$ & ES & Microinjection \\
\hline Thomas et al 1986 & $1: 100$ & Somatic & Microinjection \\
\hline
\end{tabular}

Table 8.1 - Studies where a high targeting frequency (ratio of HR compared to random insertion) was achieved without positive selection.

It therefore seemed appropriate to determine the rate of gene targeting at the Epo locus in a large series of zygote injections, in order to determine whether this would be a feasible approach to modifying the mouse genome.

In this chapter, Section 8.2 describes the demonstration that HR had occurred in the Epo$\mathrm{TAgH}^{\mathrm{H}}$ mice. Section 8.3 is concerned with the timing of that event. Section 8.4 describes the phenotype of heterozygous and homozygous Epo-TAg mice, and experiments to determine whether these mice, in which both copies of the Epo gene were modified constituted an Epo "knockout". Section 8.5 is concerned with determining the extent to which the Epo-TAg DNA was isogenic to the target locus. Section 8.6 describes a series of experiments designed to determine the targeting frequency in an experiment similar to that used to generate Epo-TAg mice.

\subsection{EVIDENCE FOR HR IN THE EPO-TAgH LINE}

The 134.3LC (Epo-TAgH) line was derived from a female founder 134.3, which bore 5 litters. In these 54 offspring a single mouse was transgenic (191.10). This mouse, which was also female, gave rise to six offspring. Amongst these offspring two different patterns were evident on Southern analysis; the first was a high copy integration (134.3HC) indistinguishable from that observed in 191.10. However the second pattern was a single copy integration. This was shown to have replaced one of the two native Epo genes. The 
first suggestion that this had occurred was that the $4.4 \mathrm{~kb} \mathrm{Bgl} \mathrm{II} \mathrm{fragment} \mathrm{containing} \mathrm{the}$ endogenous Epo gene was reduced in intensity on Southern hybridization of DNA from mice heterozygous for the transgene. The mice had a mild anaemia and in RNase protection assays the level of Epo mRNA in the kidneys of acutely anaemic Epo-TAgH mice appeared to be lower than in non-transgenic mice or other Epo-TAg mice with the same haematocrit.

Although these observations suggested that a homologous integration might have occurred, further restriction mapping was clearly required to confirm this. When Epo-TAgH DNA was subjected to restriction enzyme digestion with Bgl II, EcoRI and HindIII and hybridised to probes lying beyond the most peripheral sites in the transgene for those enzymes, no transgene-specific end fragments could be detected. More importantly, six enzymes were identified which enabled positive identification of a transgene-specific restriction fragment which was in each case exactly the length predicted for homologous insertion (Figure 8.1).

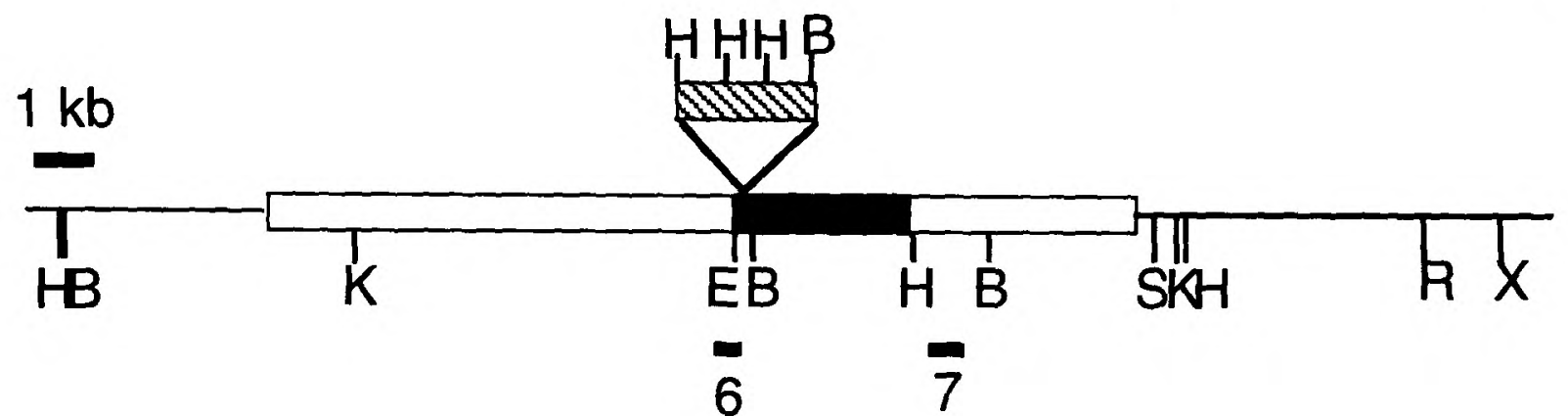

\begin{tabular}{|l|c|c|c|c|c|c|}
\cline { 2 - 7 } \multicolumn{1}{c|}{} & \multicolumn{2}{c|}{ Probe 6 } & \multicolumn{4}{c|}{ Probe 7} \\
\cline { 2 - 7 } \multicolumn{1}{c|}{} & BamH1 & Hind3 & Kpn1 & Eag/Sal & Eag/EcoRV & Eag/Xho \\
\hline Epo & 13.7 & 17.1 & 15.0 & 8.5 & 14.0 & 15.5 \\
\hline Epo-TAgH & 16.4 & 13.8 & 17.7 & 11.2 & 16.7 & 18.2 \\
\hline
\end{tabular}

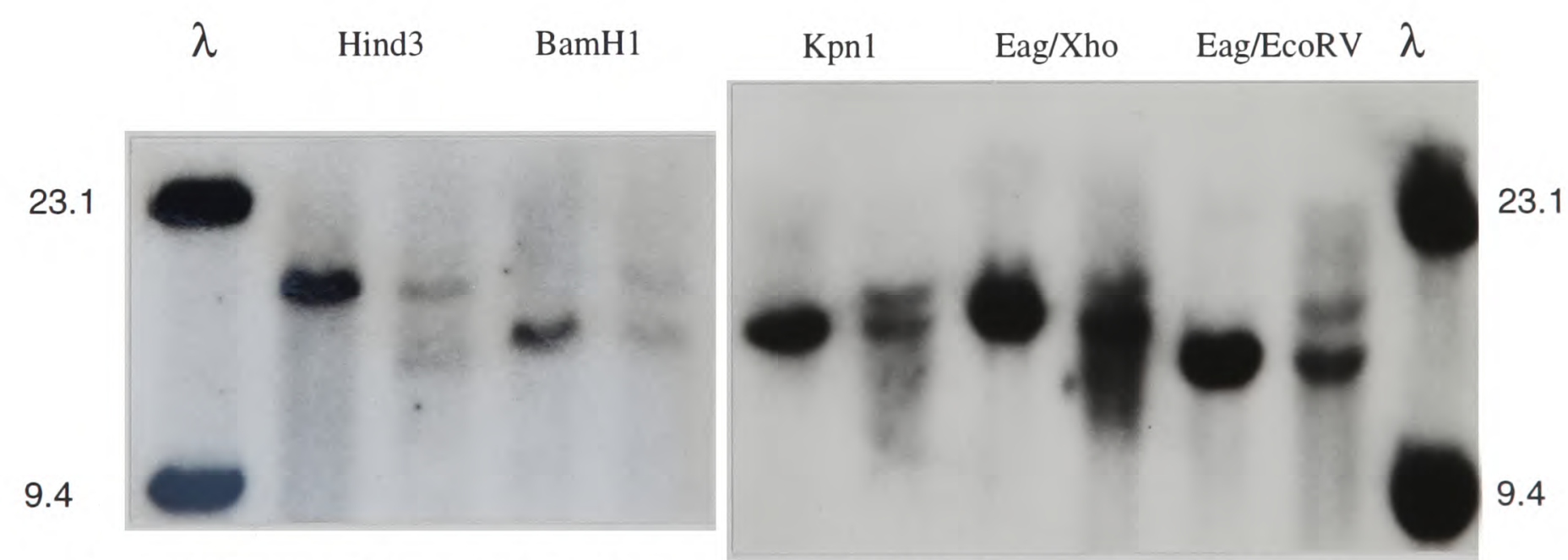

Figure 8.1 - Restriction analysis demonstrating HR. The figure shows the $\lambda 3$ a clone (open box) including the Epo gene (closed box). Flanking sequence at the Epo locus is shown as a line. Restriction sites $H=H$ indIII, $B=B$ amHI, $K=K p n I, E=E a g I, S=S a l l, R=E c o R V, X=X h o I$. The SV4O sequence is shown hatched. Lengths of predicted fragments if HR had occurred are indicated in $k b$. The Southern blots shown have lanes alternately containing DNA from non transgenic and heterozygous Epo-TAg ${ }^{H}$ mice. The size marker is $\lambda$ DNA cut with HindIII. These are consistent with the predicted lengths. Interestingly, EagI cuts the Epo-Tag ${ }^{H}$ gene less efficiently than the Epo gene, suggesting differential methylation. 
Further evidence for HR utilised the observation of a BglII polymorphism at the Epo locus. Nan mice were found to have an Epo allele ("B") in which a BglII site lying in the 3'UTR was absent. This resulted in a BglII fragment which was $250 \mathrm{bp}$ longer when probed with Probe 6 than the "A" allele present in all mice previously analysed. The Epo-TAg DNA gave rise to a $6.7 \mathrm{~kb} \mathrm{BglII}$ fragment on hybridisation to the same probe; if this showed allelic behaviour with the $\mathrm{A}$ and $\mathrm{B}$ alleles (constituting a $\mathrm{T}$ allele) it would demonstrate that HR had occurred. A cross of AB with a transgenic mouse (genotype AT or AAT) would be informative; offspring of the genotype BT would demonstrate that one native Epo allele was replaced by the $\mathrm{T}$ allele. Conversely, offspring of the genotype ABT would exclude this possibility. The results of such a cross are indicated in Figure 8.2; AB x AT gives rise to $\mathrm{AA}(4 / 19)$, AT (8/19), BT (3/19) and AB (4/19). No offspring of the genotype ABT were ever observed.

Finally, it was possible to breed mice which were homozygous for the $T$ allele, further confirming that the $\mathrm{T}$ allele showed allelic exclusion with the native Epo gene (Figure 8.3).

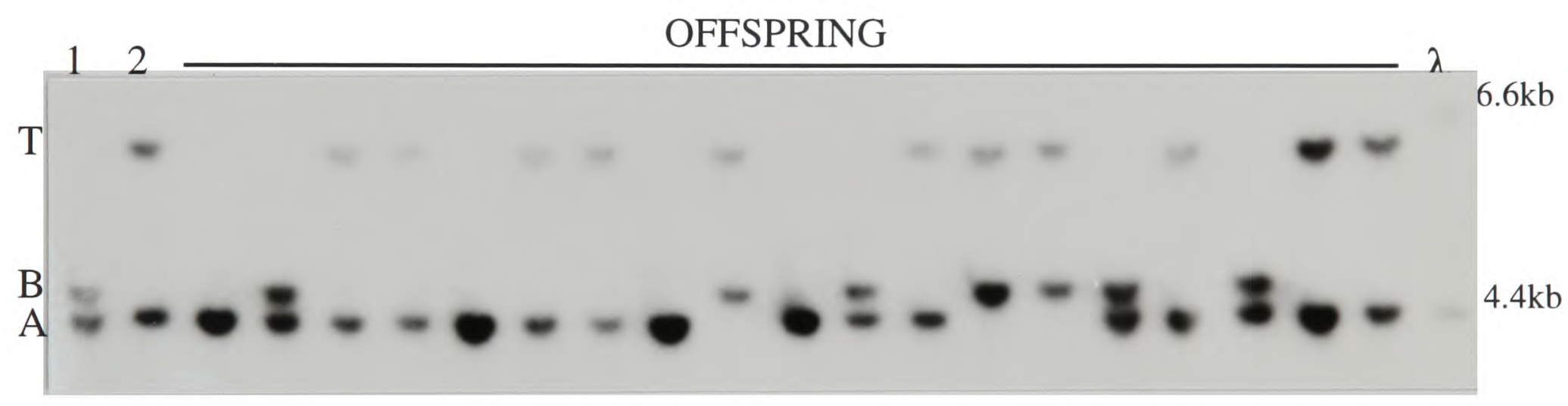

Figure 8.2 - Southern analysis of the offspring of a cross between $A B$ and AT (lanes 1 and 2). Offspring were of genotypes $A A, A B, A T$ and $B T$ demonstrating that $T$ is allelic with $A$ and $B$. DNA was digested with BglII and hybridised to Probe 6.

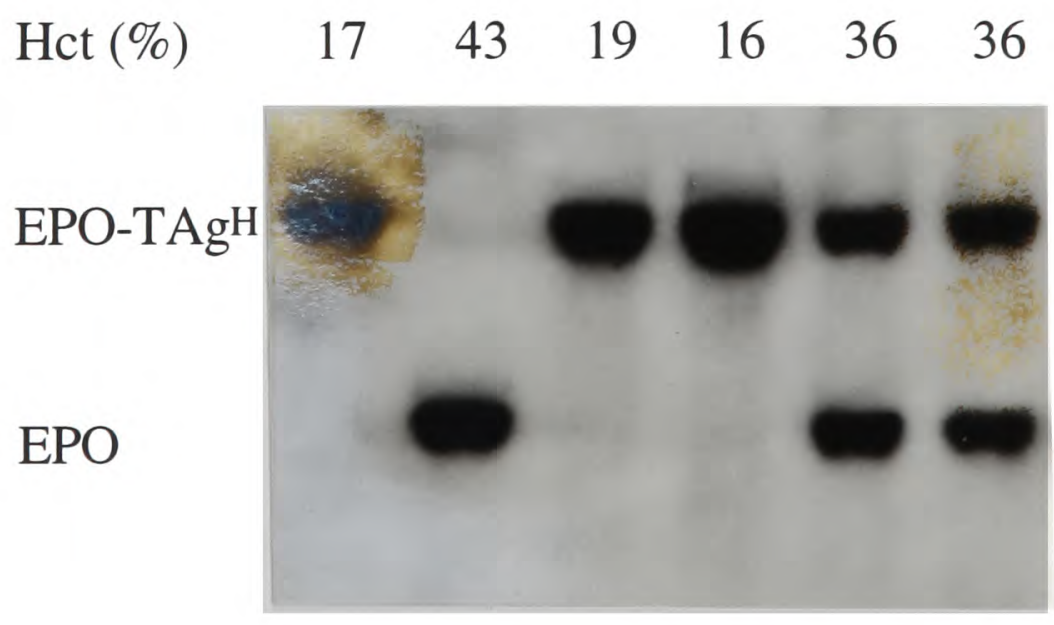

Figure 8.3 - Allelic exclusion of the Epo-TAg ${ }^{H}$ (T allele) and the native Epo gene. Southern blot of offspring of AT $x$ AT showing TT offspring. The haematocrit of each mouse is also given. 


\subsection{TIMING OF THE RECOMBINATION EVENT}

Having demonstrated that this recombination had occurred, it was important to investigate the timing of this event. The low frequency of transmission of either transgene to the offspring of 134.3, taken with the subsequent Mendelian transmission of Epo-TAgH and 134.3HC suggests that 134.3 was a germline mosaic for both transgenes. In addition, on $B g l I I$ digestion and Southern analysis of DNA from the tail of 134.3 , the intensity of the transgene-derived band (corrected for the intensity of that from the native Epo gene) was approximately five-fold less than in 191.10 and subsequent $134.3 \mathrm{HC}$ mice. This is consistent with somatic cell mosaicism, but at a level (at least in the tail) that was probably higher than in the germline.

In contrast, over $50 \%$ of the offspring of 191.10 bore the Epo- $\mathrm{TAg}^{\mathrm{H}}$ transgene suggesting that half the germ cells of 191.10 bore the Epo-TAgH transgene. It is reasonable to conclude that the ovum giving rise to 191.10 bore the Epo-TAgH transgene. The recombination presumably occurred before (or at the time of) production of this gamete. That 134.3 was a mosaic means that the Epo-TAg DNA must have integrated late in $\mathrm{S}$ phase of the first cell cycle or some time thereafter. The targeted event and the integration of $134.3 \mathrm{HC}$ could have occurred at the same time. Alternatively, at some subsequent point recombination between the $134.3 \mathrm{HC}$ array and the native Epo gene might have taken place; for instance at the meiosis giving rise to the gamete which subsequently became 191.10.

In order to distinguish these two possibilities, the presence of an $11.2 \mathrm{~kb}$ band on a double digest with Eagl and Sall was sought in 134.3 tail DNA. This digest was hard to interpret because of bands arising from the 134.3HC array, and it was also difficult to obtain complete digestion with this pair of enzymes. However, such a band did appear to be present, suggesting that the recombination was detectable in the tail of 134.3 , and had therefore occurred prior to formation of the gamete which was subsequently 191.10.

The possibility that the $134.3 \mathrm{HC}$ integration had occurred at a site close to the Epo locus was also tested. A $134.3 \mathrm{HC}$ transgenic was crossed with a mouse of genotype $\mathrm{AB}$, this demonstrated that the inheritance of 134.3 did not segregate with either allele; i.e the site of the $134.3 \mathrm{HC}$ integration was not linked to the Epo allele. 


\subsection{PHENOTYPE OF EPO-TAg ${ }^{\mathrm{H}}$ MICE.}

Epo-TAgH mice have $2.7 \mathrm{~kb}$ of viral sequence inserted in the 5'UTR of the Epo gene. This viral sequence includes polyadenylation signals. This insertion might be expected to have two effects. Firstly, the marker gene would be in the context of all cis-acting sequences. It would be expected to be expressed in a very similar manner to the Epo gene; although clearly the post-transcriptional events might be quite different. The RNA analyses described in Chapter 4 demonstrate that the levels of transgene and native Epo mRNA were similar. However, as discussed in Chapter 6, the level of expression in the liver was reduced compared to that of the native gene in acutely anaemic mice. The most likely explanation for this was considered to be the distancing of important regulatory sequence (perhaps the 3' enhancer) from the Epo promoter. The second effect would be to reduce the production of Epo. Indeed the Epo-TAg gene might not produce Epo at all, since the Epo sequence lies 3 ' to the polyadenylation signals of the SV40 T Ag gene. The phenotype of mice with a relative, or absolute, deficiency in Epo would be important in understanding the role of Epo in normal animals. Such "knockout" experiments have yielded useful information in understanding the role of many gene products (Capecchi 1989).

\subsubsection{Phenotype of lieterozygotes}

The anaemia of Epo-TAgH heterozygous mice suggested that the Epo-TAg gene was not producing Epo normally. The mild anaemia of these animals could be entirely due to the loss of one copy of the Epo gene, with a consequent reduction in basal Epo secretion. Alternatively, it is possible that expression of SV40 T Ag in the Epo-producing cells would influence the expression of the Epo gene. Some evidence for the latter possibility comes from the observation that 188 .1LC animals were significantly anaemic when compared to their non-transgenic littermates (Table 8.2). The possibility that in 188.1LC the mouse Epo gene had been replaced by HR was excluded by crossing with $A B$ heterozygotes, and thus demonstrating that the ABT genotype was possible in this line. This suggests that SV40 T Ag expression in Epo-producing cells alters expression of the native Epo gene.

\begin{tabular}{|l|c|c|}
\cline { 2 - 3 } \multicolumn{1}{c|}{} & $188.1 \mathrm{LC}$ & non $\mathrm{tg}$ \\
\hline $\mathrm{n}$ & 19 & 18 \\
\hline mean & $35.4 \%$ & $38.0 \%$ \\
\hline s.d. & $3.2 \%$ & $3.5 \%$ \\
\hline
\end{tabular}

Table 8.2 - Haematocrits of 188.1LC transgenic mice and non-transgenic littermates. The Epo-TAg transgenic mice were significantly anaemic ( $p=0.023$, Student's $t$ test). 


\subsubsection{Phenotype of homozygotes}

An important issue was whether homozygous Epo-TAgH mice would be viable. Matings of heterozygous Epo-TAgH mice produced homozygous offspring at the predicted frequency. These homozygous mice were observed to have a severe anaemia. Males were fertile but female mice were generally not.

\subsubsection{Do Epo-TAg ${ }^{\mathrm{H}}$ mice constitute an Epo "knockout"?}

Although these mice were anaemic, and had insertions in the 5' UTR of both copies of the Epo gene, the Epo coding sequence had not been altered. In order to determine whether Epo coding sequence was transcribed, RNA was analysed by RNase protection assay, using the MsEpo probe. RNA was extracted from kidney and other tissues of a homozygous Epo-TAg mouse, which had been rendered more anaemic with phenylhydrazine. The analysis clearly demonstrated a similar level of the 65 base pair and 148 base pair species transcribed from $5^{\prime}$ and $3^{\prime}$ to the SV40 $\mathrm{T}$ Ag coding sequence. Thus most, if not all, the Epo-TAg transcripts run through the SV40 polyadenylation signals into the Epo coding sequence (Figure 8.4).

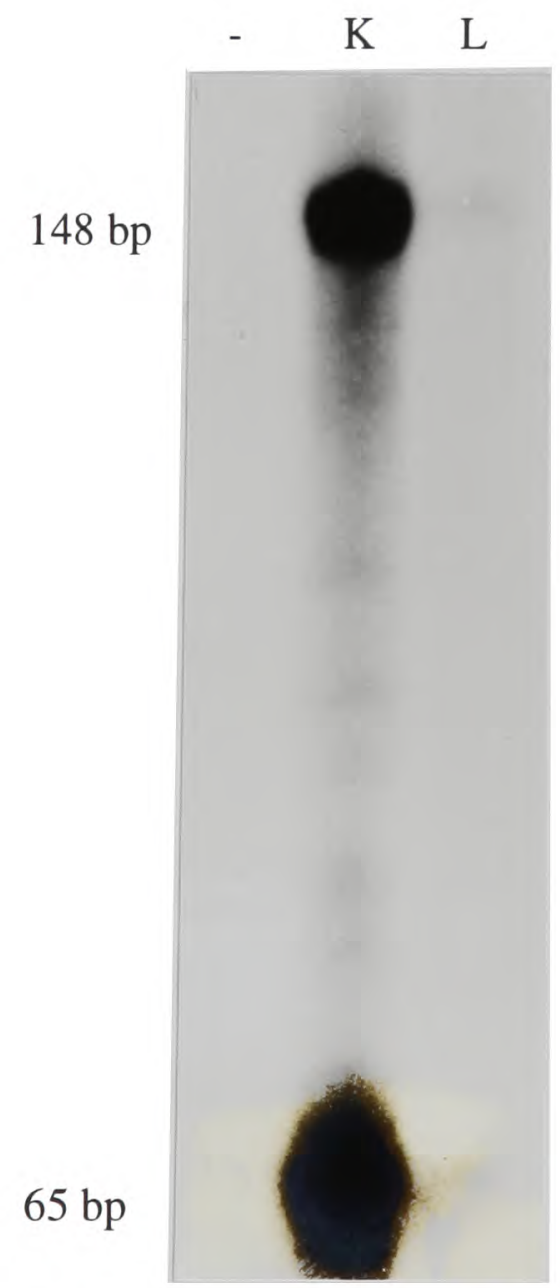

Figure 8.4 - RNase protection assay analysing transcription of the Epo-TAg ${ }^{H}$ gene in the kidney $(K)$ and liver $(L)$ of a phenylhydrazine-treated Epo-TA ${ }^{H}$ homozygote. This probe gives rise to a 65 bp protected fragment corresponding to the Epo 5' UTR and SV4O sequence. In addition there is a 148 bp species corresponding to the Epo sequence lying 3' to the SV4O
polyA sites. 
Next, serum from phenylhydrazine-treated homozygous Epo-TAgH mice was assayed for Epo by RIA. Epo was present at a level of 56 and $60 \mathrm{mIU} / \mathrm{ml}$ in two different homozygous mice treated in this fashion (final haematocrit 10 to 12\%). Thus immunoreactive Epo was present, but at a level approximately 100 fold lower than would have been observed in normal mice.

These mice thus have detectable, but severely reduced levels of Epo. That they are viable raises the possibility that mice with complete Epo deficiency might also be viable. This would demonstrate that red blood cell production could occur in the absence of Epo. Phenotypic observations would also provide insight into the functional significance (if any) of Epo expression in sites such as the brain and testis, where a local role might be important. It is not possible to address this issue by studies of homozygous Epo-TAgH mice, since they do have a capacity for Epo production, albeit at a reduced level. However, several observations can be made on the phenotype of these animals.

\subsubsection{Effect of reduced Epo production on erythropoiesis}

As an initial assessment of haemopoiesis in homozygous Epo-TAg ${ }^{\mathrm{H}}$ mice, bone marrow cytospins were examined by $\operatorname{Dr} \mathrm{S}$. Watt. As expected, there was a clear reduction in the number of nucleated erythroid cells. No other significant abnormalities were noted.

In order to investigate whether erythropoietic factors might be induced by anaemia when Epo was relatively deficient, serum from a homozygous Epo-TAgH mouse was assayed using an in vitro bioassay (kindly performed by Roz Bill). The assay technique employed measures thymidine uptake by spleen cells isolated from an acutely anaemic mouse, and was adapted from the assay described by Krystal (Krystal 1983). No difference was observed between thymidine incorporation in the presence of the two sera. As described previously, the effect of mouse serum in this assay (which would contain approximately 6-20 mIU/ml of Epo) was more than the effect of $50 \mathrm{mIU} / \mathrm{ml}$ recombinant mouse erythropoietin. This assay did not demonstrate the presence of other growth factors at a different level from that found in a normal mouse.

These Epo deficient mice may constitute a useful tool to understand some of the effects of severe anaemia. In contrast to other models the level of Epo is not elevated. For instance, these mice are currently being used to confirm the previous observation (Raja et al. 1986) that anaemia appears to increase iron absorption by the gut, independent of increased Epo levels or increased iron utilisation. 


\subsubsection{Other effects of reduced Epo levels}

As a crude assessment of phenotypic effects of the insertion of viral sequence at the Epo locus in Epo-TAg mice, the size of several organs (wet weight as a fraction of the weight of the mouse) were compared (Table 8.3). There was no difference in heterozygotes or homozygotes in the size of the kidneys, liver or spleen. The heart was substantially hypertrophied in the homozygotes; presumably as an adaptive change to the chronic anaemia.

\begin{tabular}{|l|c|c|c|}
\cline { 2 - 4 } \multicolumn{1}{c|}{} & Homozygote & Heterozygote & NonTG \\
\hline Kidneys & $1.6+/-0.3$ & $1.6+/-0.3$ & $1.6+/-0.4$ \\
\hline Liver & $4.6+/-0.3$ & $4.6+/-0.4$ & $4.6+/-0.5$ \\
\hline Heart & $0.6+/-0.1$ & $0.4+/-0.0$ & $0.4+/-0.0$ \\
\hline Spleen & $0.2+/-0.1$ & $0.3+/-0.0$ & $0.3+/-0.0$ \\
\hline
\end{tabular}

Table 8.3 - The weight of various organs as a percentage of body weight (mean $+/$ - standard deviation, $n=8$ in each group). The only significant difference was the increased mass of the heart in homozygotes.

At a microscopic level, the number of cells in the renal interstitium was increased in homozygotes (see Chapter 7); this is likely to be due to SV40 T Ag expression in these cells, rather than an effect of the anaemia or depressed Epo production.

That the Epo- $\mathrm{TAg}^{\mathrm{H}}$ homozygotes did not have marked abnormalities apart from anaemia suggests that Epo production in ES cells (Schmitt et al. 1991), the brain (Masuda et al. 1994), the testis (Tan et al. 1992) and other organs is not of major functional significance. However, to be sure of this it would be necessary to generate an Epo "knockout".

In limited experiments it was possible to segregate a litter of Epo-TAg ${ }^{\mathrm{H}}$ homozygotes and heterozygotes at birth on the basis of pallor. This strongly suggests that foetal Epo production is important in regulating foetal erythropoiesis in the mouse, even though maternal Epo can cross the placenta (Koury et al. 1988a).

\subsection{DEGREE OF ISOGENICITY IN THE EPO-TAG EXPERIMENT}

As discussed above, the degree of isogenicity between target and incoming DNA is likely to influence the targeting frequency. The observation of recombination in Epo- $\mathrm{TAg}^{\mathrm{H}}$ raises the question of whether the original Epo-TAg DNA was isogenic to that of the recipient zygote.

The only way to be certain of sequence divergence between strains in the region contained in the Epo-TAg construct would be to sequence the entire $16.5 \mathrm{~kb}$. The recipient embryo would have had two Epo alleles; one derived from CFLP (non-inbred) and one from either C57BL/10 or CBA. This has not been performed. Instead, limited studies were undertaken, summarised in Figure 8.4. First, a 70 dinucleotide (CA) repeat lying 3 ' to the gene was 
amplified by PCR from several strains; C57BL6, C57BL10, C3H/HeH, CBA, 101/J, 129/ Ola and an Epo-TAgH homozygote. The product obtained from each mouse strain was then analysed by PAGE followed by ethidium staining. No difference in length was found in any strain. Secondly, a 200 bp region spanning the $3^{\prime}$ UTR and the $3^{\prime}$ enhancer was sequenced directly from PCR product from a reaction utilising a biotinylated primer. No differences in sequence were detected, except for a 17 bp deletion in the 3'UTR in 101/J and 129/Ola (which included the $B g l I I$ site). Thirdly, $300 \mathrm{bp}$ lying slightly further 3 ' to the polyadenylation site was sequenced from an Epo-TAgH homozygote, $\mathrm{CBA}, \mathrm{C} 3 \mathrm{H} / \mathrm{HeH}, \mathrm{Balb} / \mathrm{c}$ and 129/Ola (Appendix 5). The only difference detected was a single nucleotide addition in 129/Ola. Finally, a panel of six restriction enzymes (BglII, Mspl, Pst 1, Rsal, Sacl and Taql) was used in conjunction with a probe lying 5 ' to the gene (Probe 4, Figure 2.1, p.46) to analyse CBA, C3H/HeH, C57BL6, C57BL10, Balb/c and Epo-TAgH. No RFLP's were identified in this region. Thus it remains unknown to what extent the incoming and native DNA were identical; but it appears that there is very little variation between the mouse strains represented in the library and in the injected zygote at the Epo locus.

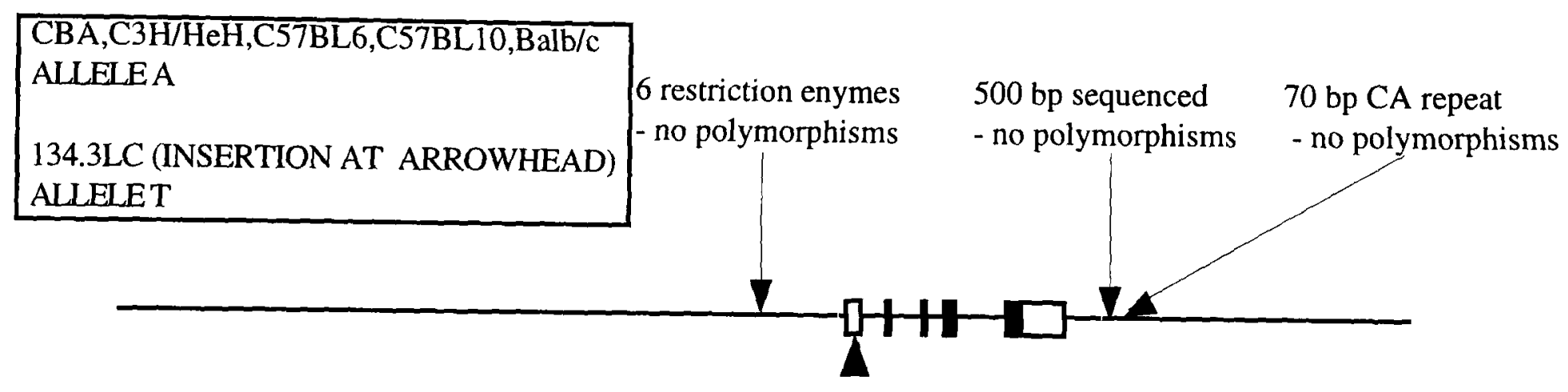

101/J, 129/Ola ALLELE B

13 bp deletion with single base insertion loss of $\mathrm{Bgl} 2$ site

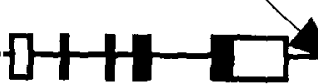

Figure 8.4 - Determination of the degree of variation at the Epo locus. Various inbred strains, including those contributing to the genetic background of the injected zygotes and those which may have been represented in the $\lambda$ library, were analysed with restriction enzymes, by sequence analysis and the length of a $C A$ repeat. These were identical in each of these strains and in Epo-TAg ${ }^{H}$ (the recombinant allele). By contrast, two differences from this A allele were found 3' to the gene in 101/J and 129/Ola (these two inbred strains were derived from a common source originally). 


\subsection{RATE OF HR AT THE EPO LOCUS.}

The experiment undertaken was to inject zygotes with a construct which contained $16 \mathrm{~kb}$ of homology to the mouse Epo locus, and was derived from the same $\lambda$ clone as Epo-TAg and Epo-M. This construct, Epo-D contained a linker between an Eagl and BamHI site thus removing $456 \mathrm{bp}$ which included the first exon. Succesful recombination would result in a disabling deletion of the Epo gene and a null allele.

The Epo-TAgH allele had been the result of HR with DNA from the $\lambda$ clone (the Epo-TAg construct). In the absence of precise knowledge concerning the mouse strain from which the $\lambda$ clone originated, this recombinant allele offered the most attractive substrate for recombination with the Epo-D construct.

In order to allow the diagnosis of HR, Epo-D contained EcoRV and Xhol restriction sites (Appendix 2). In the event of recombination, these would result in fragments of 14.0 and $15.5 \mathrm{~kb}$ hybridising to probe 6 . To facilitate diagnosis of recombinant events, the experiment incorporated the possibility of detection by allele substitution. DNA was injected into zygotes which were heterozygous at the Epo locus, having a maternal ' $A$ ' Epo allele and a paternal ' $\mathrm{T}$ ' Epo allele. These zygotes were generated by mating homozygous Epo-TAgH males (genotype TT) with females which were $F_{1}$ crosses of CBA and C57BL10 (genotype AA). When DNA from the embryos was digested with $B g l I I$ and hybridised to Probe 6 this would yield a fragment of $4.0 \mathrm{~kb}$ (A allele) and $6.7 \mathrm{~kb}$ ( $\mathrm{T}$ allele). The injected DNA would give a $3.6 \mathrm{~kb}$ fragment (D). Transgenic embryos would therefore give all three fragments; if HR were to occur then the genotype would be AD or TD, due to substitution of one allele (Figure 8.5). This method would thus also identify whether the recombination was with the maternal or paternal allele.

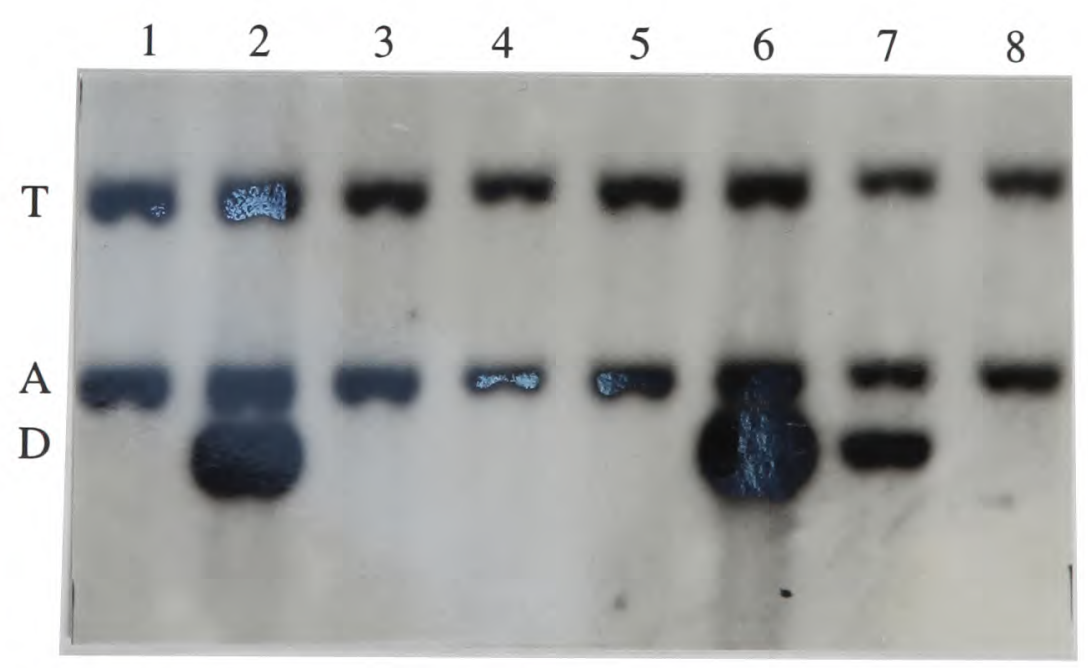

Figure 8.5 - Southern analysis of embryos injected with Epo-D. DNA was digested with BglII and hybridised with Probe 6. The recipient zygotes were heterozygous at the Epo locus (genotype AT). Integration of Epo-D results in the genotype ATD, as seen in lanes $2,6,7 . H R$ would give rise to embryos of genotype TD or $A D$. 
As discussed above, the integration of the exogenous DNA in the 134.3 embryo was late, resulting in a mosaic. This might have been important in allowing HR to occur. Half the current series of injections were therefore performed at a "late" time point. Some injections were into the female pronucleus.

\subsubsection{Results}

2,637 zygotes injected with Epo-D DNA were implanted in pseudopregnant females. 1,096 viable embryos resulted, and were subjected to DNA analysis. Of these 200 were transgenic. None of these transgenic embryos had lost the A or T alleles suggesting that HR had not occurred. The effect of timing of injection and site of injection on transgenic rate is summarised in Table 8.4; no effect was discernible.

\begin{tabular}{|l|c|c|c|}
\hline Injection site \& time & Transferred & Viable & Transgenic \\
\hline Male PN, early & 1485 & 558 & $108(19)$ \\
\hline Male PN, mid & 96 & 27 & $3(11)$ \\
\hline Male PN, late & 965 & 468 & $81(17)$ \\
\hline Female PN & 91 & 27 & $8(30)$ \\
\hline All injections & 2637 & 1080 & $200(19)$ \\
\hline
\end{tabular}

Table 8.4 - The number of zygotes injected with Epo-D DNA and transferred is given. At day 14 to 18 of pregnancy the recipient females were sacrificed, resulting in the number of viable embryos shown. DNA was extracted and analysed for the presence of the Epo-D transgene. The transgenic rate (given as a percentage in parentheses) was very similar in the two larger groups. $P N=$ pronucleus. Early = at most one hour following formation of $P N$. Late = at least 8 hours after PN formation.

Table 8.5 summarises the effect of the time of injection on apparent transgene copy number. Lower apparent copy numbers might reflect either lower copy transgene arrays, or mosaicism. In embryos injected late there appeared to be an increased frequency of transgenic embryos with apparent copy number of less than one. These embryos were clearly mosaics.

\begin{tabular}{|l|c|c|c|c|c|c|}
\cline { 2 - 7 } \multicolumn{1}{c|}{} & \multicolumn{6}{c|}{ Apparent copy number } \\
\hline Injection time & $<1$ & 1 & 2 & 4 & 8 & $>8$ \\
\hline Early & $3(3)$ & $12(11)$ & $20(19)$ & $24(22)$ & $28(26)$ & $21(19)$ \\
\hline Late & $7(8)$ & $5(6)$ & $11(12)$ & $20(22)$ & $23(26)$ & $23(26)$ \\
\hline
\end{tabular}

Table 8.5 - Distribution of apparent transgene copy number in Epo-D transgenic embryos as a function of injection time. Numbers in parentheses are percentages.

There are several ways in which the above analysis could miss HR if it had occurred. Firstly, it is possible that DNA integration would have occurred at two separate sites, one of which involved HR at the Epo locus. Secondly, the integration might have occurred late, resulting in a mosaic (eg a mixed genotype of AT and DT). Thirdly, during the recombination process it is possible that the DNA from the native Epo allele would integrate elsewhere. Fourthly, it is possible that Epo-D integrated at the Epo locus by an insertion type mechanism, ie without replacing the native gene. All four possibilities would result in the genotype 
ATD on the Southern analysis performed. The first three would give rise to the desired offspring on breeding. The first two both applied to the recombination observed in 134.3. The third and fourth have been documented in analagous situations (Adair et al. 1989). None of these possibilities are easily subject to analysis. In order to examine the second and third possibilities all low copy Epo-D embryos were digested with EcoRV and analysed by Southern analysis with a probe lying $2 \mathrm{~kb}$ external to the 3 ' end of the $\lambda 3 \mathrm{a}$ clone (Appendix 3 ). All the embryos showed the anticipated large ( $>23 \mathrm{~kb}$ ) fragment, with no evidence of $\mathrm{HR}$ (which would give rise to a $13.5 \mathrm{~kb}$ fragment).

This result suggests that the frequency of reliably detectable recombination events is too low to make this a viable system for modifying the mouse genome.

\subsection{CONCLUSIONS}

The attempt to obtain HR at a workable rate by direct zygote injection was unsuccesful. The reason for the experiment was to determine whether optimising two factors (length and homology of incoming and target DNA) would achieve a workable rate of recombination. However, it appears that recombination is still too infrequent for this to be a feasible method of modifying the mouse genome.

The use of more extensive homology makes the diagnosis of HR more difficult, since the distance from modification to flanking sequence will inevitably be increased. In the current experiment this distance was $9 \mathrm{~kb}\left(5^{\prime}\right)$ or $7 \mathrm{~kb}\left(3^{\prime}\right)$. The allele exchange system used to screen for HR is a novel strategy which may be of use in similar experiments in the future. More extensive DNA from the Epo locus has been isolated from a cosmid library (Appendix 3). This would allow further attempts at HR using longer homologous sequence.

Recently modifications to standard PCR techniques have been described allowing amplification of much longer target sequences (Barnes 1994; Cheng et al. 1994). This may well overcome some of the difficulties in screening for HR. Using this technique, I have amplified a $7 \mathrm{~kb}$ fragment extending from the SV40 T Ag sequence to sequence $3^{\prime}$ to the original $\lambda$ clone in an Epo-TAgH homozygote. This indicates the potential for such techniques to assist in the diagnosis of recombination events. Sequence information external to the $\lambda$ clone has been obtained, and could be used for long PCR (Appendix 3).

However, it seems likely that if direct zygote injections are to offer an alternative to stem cells for modifying the mouse genome a strategy to enhance HR would be required. The Epo-TAg experiment may have resulted in recombination through chance alone. 
Alternatively, some other feature of the experiment may have favoured recombination. SV40 $\mathrm{T} \mathrm{Ag}$ is known to have helicase properties (Stahl et al. 1986), alters the cell cycle and can bind to the SV40 sequence in Epo-TAg (Tijan, 1981). In this context it is interesting to speculate that the transient expression of SV40 T Ag by injected zygotes in the original Epo-TAg experiment may have favoured recombination.

In terms of investigation of Epo gene expression, the Epo-TAgH line demonstrates that a major reduction in Epo gene expression has little phenotypic effect, except for anaemia. This suggests that normal expression of the gene in tissues such as brain and the gonads is not necessary for normal development and fertility. In addition, it raises the possibility that sufficient erythropoiesis for life could be maintained in the complete absence of Epo. 


\section{Concluding remarks}

The experiments discussed in Chapters 4 and 5 of this thesis have led to the identification of the renal cell population expressing the Epo gene. They are the Type 1 interstitial cells of the renal cortex and outer medulla. These cells are fibroblastoid. The transgenic strategy used to identify these cells directed expression of a viral oncogene to this specific population. Although comparison with other studies is difficult, it appears likely that this population is rather resistant to the effect of the oncogene (SV40 T antigen); however it is clear that they are not terminally differentiated, since the population can be expanded. One striking feature of renal disease is that there is a relative deficiency of Epo production. Experiments described in Chapter 7 suggest that these cells alter their phenotype in the context of renal injury. They appear to become more "myofibroblastoid" and as a population have a reduced, or absent, ability to express the Epo gene. However, in each of the models used many other factors were altered. There was a marked immune infiltrate in the interstitium and both local oxygen supply and consumption would be altered. The complexity of these changes will be hard to investigate in vivo, and it remains an important goal to obtain Epo-producing cell lines from the kidney. This has not yet been achieved.

In the liver, experiments in Chapter 6 demonstrate that Ito cells express the Epo gene. These cells have important similarities to the Type 1 interstitial cells of the kidney. Intriguingly, in response to injury or in culture they undergo a phenotypic change to a myofibroblastoid phenotype. This forms an interesting parallel with the observed response of the renal Type 1 interstitial cells to injury.

The restriction of Epo gene expression to these cells and hepatocytes is striking. Some information concerning the mouse cis-acting sequence responsible for this cell-specific expression is provided by the transgenic experiments described in Chapter 4. A major area for future investigation will be the definition of the protein-DNA interactions responsible and the way that they interact with the oxygen-sensing mechanism. Many other important issues, such as the relationship between local oxygen tension and Epo gene activation in vivo, present interesting challenges.

The occurrence of homologous recombination following direct zygote injection of plasmid DNA appears to have been a chance occurrence of an event that is too infrequent to be used to generate further modifications of the mouse Epo locus. While this is unfortunate, the Epo-TAg ${ }^{\mathrm{H}}$ mouse, in which the initial recombination occurred, provides an important model of relative Epo deficiency. That these mouse (which constitute an Epo "knockdown") are 
viable and fertile raises the possibility that Epo is not crucial for survival. It will be intriguing to know whether other factors can support erythropoiesis in the complete absence of Epo. It will also be interesting to determine whether Epo has a significant role in non-erythroid tissues. Epo mRNA is quite widely distributed (see Chapter 4). Whether this expression is functionally important remains to be determined.

Another line of inquiry (Chapter 3) was concerned with the possibility that an oxygensensing mechanism capable of controlling gene transcription would not be restricted to Epo-producing cells and control of the Epo gene. It appears certain that an oxygen-sensing mechanism closely related (or identical) to that regulating Epo production is present in most (possibly all) mammalian cells in tissue culture. Several genes appear to be controlled in this fashion; the physiological significance of this remains to be determined. Many important questions remain. How does this system interact with other regulatory systems? Is it essential for cell viability, or could it be mutated? Is it operating continuously in nonEpo producing cells in vivo? 


\section{Acknowledgements}

The work described in this thesis is my own. However, I am greatly indebted to many individuals for their practical assistance and support; most importantly to Dr P. Ratcliffe, in whose laboratory and under whose guidance these studies were performed. Dr C. Pugh was generous and patient in instructing me in many of the techniques used, and was closely involved in the design of a number of the experiments. Lynn Nicholls gave invaluable technical assistance.

I would like to thank the following for their part in collaborative experiments. Dr D. Ferguson (electronmicroscopy); Prof. M. Johnson, Dr M. Osmond, Brendan Doe and Sue Pickering (transgenic experiments); Prof. C. Bauer and Dr H. Marti (co-culture experiments); Dr P. M. Cotes (Epo assays); Dr J. Iredale (liver cell separation).

The following were also generous with their advice and help. Dr K. Gatter, Dr D. Davies, Dr. K. Fleming, and Andrew Herryet (histology); Dr C.Wood and Margaret Jones (immunostaining); Roz Bill (Epo bioassay), Dr S. Watt (mouse bone marrow), Dr C. Tan, Dr J. Firth, Dr J. Gleadle, June Shultz, Kay Yeates, Sylvia Bartlett and Geoffrey Maxwell. Dr J. Gannon, Prof. B. Kaissling, Dr A. Woolf and Dr S. Povey each provided valuable reagents.

I am grateful to the MRC for the Training Fellowship which enabled me to do this work. Finally, I would like to thank my wife for her tireless good-nature and moral support. Without them this research would not have been possible. 


\section{Appendix 1}

\section{THE EPO 3' ENHANCER IN MOUSE AND MAN}

\begin{tabular}{|c|c|c|c|c|c|}
\hline & & & IYA & & \\
\hline MOUSE & CTGGCAACAG & CTGAAATCAC & CAACCAGACT & CCTGGCTTGT & СТСТСТTСАТ \\
\hline $1 \mathrm{AN}$ & $t \cdot a \cdot \cdots \cdot g \cdot a$ & $\cdots \cdots c \cdot \cdots$ & ..tat.... & $\cdot t \cdots \cdots \cdot t \cdot$ & $\cdots g \cdot t \cdot \cdots \cdot$ \\
\hline $\begin{array}{l}\text { MOUSE } \\
\text { MAN }\end{array}$ & GACTGTACAC & ACCACACAAC & TCTCCTAGCT & GTACCTCACC & CCATCTGGTC \\
\hline & & $\cdots \cdot$ & $\cdots \cdots$ & $\cdots \cdots$ & $\cdots$ \\
\hline MOUSE & GCAAGGCATC & & & & 1 Apa 1 \\
\hline $\mathbf{M A N}$ & $\ldots \ldots \ldots$ & AGATCTGGGA & AACCAGAGGT & GGAGGGGGTT & GGGCCCTACG \\
\hline & 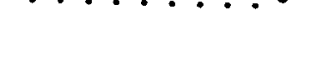 & $\bullet g \bullet \operatorname{c} \bullet \bullet \cdot$ & $\bullet \operatorname{tg} \bullet \operatorname{g} \bullet \bullet$ & $\cdots \cdots \cdots \cdot c \cdot$ & $\cdots \cdots \cdots \cdots$ \\
\hline & 11 & 21 & 31 & 41 & 51 \\
\hline MOUSE & TGCTGCCTCG & CATGGCCCGG & CTGACCTCTT & GACCCCTCTG & GGCTTGAGGC \\
\hline MAN & $\cdots \cdots t \bullet \cdot a$ & $\cdot$ ca $\cdots \cdot t \cdot t$ & $\cdots \cdots \cdots \cdot c$ & $\cdots+$ ta $\cdot c \cdot$ & $\cdot \cdot c \cdot \cdots \cdot$ \\
\hline & 61 & 71 & 81 & 91 & 101 \\
\hline MOUSE & САСАATACCT & GCCCACGCTA & GTCAATAAGC & AGGCTCCATT & CAAGGCTGTC \\
\hline MAN & $\cdots \cdots$ gct $\cdots$ & $\cdots \cdot \cdots \cdot \bullet g$ & $\cdots \cdots \cdots \cdot g$ & $t \cdot t \cdots \cdots \cdots$ & •....ctca \\
\hline & 111 & Pvu & & & \\
\hline $\begin{array}{l}\text { MOUSE } \\
\text { MAN }\end{array}$ & TCTCAGTGGG & CAGCT & & & \\
\hline MA & $c \cdot g \cdot \bullet \cdot$ & & & & \\
\hline
\end{tabular}

Alignment of the mouse and human 3 ' enhancer region. The human and mouse polyadenylation sites are underlined. Base identity is indicated by $\bullet$ in the human sequence. A gap is inserted in the human sequence where necessary to produce alignment. .... indicates no clear homology. + indicates a polymorphism in the human sequence (either $C$ or no base). Numbering, from the first base of the Apal site, is for the mouse sequence, and is as used in this thesis when describing fragments of this sequence. Hypoxically-induced DNA binding activity was obtained by Wang and Semenza with a probe from bases 4 to 21 of the human sequence (Wang and Semenza 1993). Nucleotides 1 to 26 of the mouse sequence (Pugh et al. 1993) and 1 to 24 of the human sequence (Madan and Curtin 1993) can function as an enhancer element in transient transfection studies. Mutational analysis of the mouse sequence suggests that bases 5 to 12, 25 to 29 and 37 to 48 are functionally important (Pugh et al. 1993). Constitutive DNA binding activity has been demonstrated to nucleotides 28 to 49 of the mouse sequence (Pugh et al. 1993) and 29 to 51 of the human sequence (Wang et al. 1993). Nucleotides 32 to 45 of the human (and mouse) sequence contain a direct repeat of a steroid/thyroid hormone receptor response element (Blanchard et al. 1992). 
Appendix 2

\title{
TRANSGENE SEQUENCE AND PCR DIAGNOSIS
}

\author{
EPO-M TRANSGENE \\ Xbal \\ 1 TCTAGACTCC AGCCTTAGTT GITTCTGATC TAAATCCCTG CCTCAAGACA \\ 51 AGGATGCTCC CACCCCCCAC ACACCCCCGC CCCCAGGTGT TCCGOCGCCC \\ 101 AACTTTTCAT AGGTCAATTA TCCTTACCTC AGCCTTAGGC TTACCTTCCC \\ 151 GCAAATCTCT CAGATGCGCA CCCCCCTGGT CTACCCTCCT GGAAATCAGA \\ 201 ACCTCCCCCC CCCCAAAACC CCCCTGACGG CGTGTGTGCC TGGAACAGCC \\ 251 TGCTCCACCC CAGCAAGCAC CAGACCCAGG CGTCCTGCCC CTTGCTCTGA \\ 301 CCCCAGGTGG CCCCCACCTC TGGCGACCCC TCACGCACAC ACAGCTTCAC \\ 351 CCCCACCCCC ACCCGCGCAC GCACACATGC TGATAACATC CCCGACCCCC \\ 401 GGCCGGAGCC TRANSCRIPTION START \\ BglII $\longrightarrow$ OLIGO 4 \\ 451 ACTGTGTTCC CGAACGGACC CTTGGCAGAT CTGCCAGGGC CACCGCGTCC \\ 501 CCACTCTGCC CGCGCCCCCT GGACAGTGAC CACTTICTTC CAGGCTAGTE \\ 551 GGGTGATCTG GCCCTACAGA ACTTCCAAGG ATGAAGACTT GCAGCGTGGA \\ Sac1 \\ 601 CACTGGCCCA GCCCCGGGTC GCTAAGGAGC TCCGGCAGCT AGGCGCGGAG \\ End of exon 1 \\ 651 ATGGGGGTGC CCGG
}

Epo-M transgene. The 8 bp BgllI linker is underlined. The two primers used for PCR diagnosis are shown in blue. 


\section{EPO-TAg TRANSGENE}

Xbal

1 TCTAGACTCC AGCCTTAGTT GTTTCTGATC TAAATCCCTG CCTCAAGACA

51 AGGATGCTCC CACCCCCCAC ACACCCCCGC CCCCAGGTGT TCCGGCGCCC

101 AACTTTTCAT AGGTCAATTA TCCTTACCTC AGCCTTAGGC TTACCTTCCC

151 GCAAATCTCT CAGATGCGCA CCCCCCTGGT CTACCCTCCT GGAAATCAGA

201 ACCTCCCCCC CCCCAAAACC CCCCTGACGG CGTGTGTGCC TGGAACAGCC

251 TGCTCCACCC CAGCAAGCAC CAGACCCAGG CGTCCTGCCC CTTGCTCTGA

301 CCCCAGGTGG CCCCCACCTC TGGCGACCCC TCACGCACAC ACAGCTTCAC

351 CCCCACCCCC ACCCGCGCAC GCACACATGC TGATAACATC CCCGACCCCC TRANSCRIPTION START

401 GGCCGGAGCC ACAGTGTCCC GGGACCAACC CTGGCCGGTG GCTGTGTCTC $\mathrm{Bgl} 2$

451 ACTGTGTTCC CGAACGGACC CTTGGCAGAT CTGTGGCCTC TGAGCTATTC

501 Stu 1

CAGAAGTAGT GAGGAGGCTT TTTTGGAGGC CTAG

Epo-TAg transgene. The BglII linker is shown in red. Sequence 3' to this is from the SV4O genome. Primers used for PCR diagnosis are shown in blue.

\section{EPO-D TRANSGENE}

Xbal

1 TCTAGACTCC AGCCTTAGTT GTTTCTGATC TAAATCCCTG CCTCAAGACA

51 AGGATGCTCC CACCCCCCAC ACACCCCCGC CCCCAGGTGT TCCGGCGCCC

101 AACTTITTCAT AGGTCAATTA TCCTTACCTC AGCCTTAGGC TTACCTTCCC

151 GCAAATCTCT CAGATGCGCA CCCCCCTGGT CTACCCTCCT GGAAATCAGA

201 ACCTCCCCCC CCCCAAAACC CCCCTGACGG CGIGTGTGCC TGGAACAGCC

251 TGCTCCACCC CAGCAAGCAC CAGACCCAGG CGTCCTGCCC CTTGCTCTGA

301 CCCCAGGTGG CCCCCACCTC TGGCGACCCC TCACGCACAC ACAGCTTCAC

Eag1

351 CCCCACCCCC ACCCGCGCAC GCACACATGC TGATAACATC CCCGACCCCC BamHl

401 GGCCGCTCGA GGATATCGGA TCCAGAACAG GGACAAAATT TAGGGATTTT

892 GGGGAGATGA GGCGCTGCTC

Epo-D transgene. The linker between the Eagl and BamHI sites is shown in red. Primers used for PCR diagnosis are shown in blue. 


\section{COSMID CLONES AND MAPPING THE EPO LOCUS}

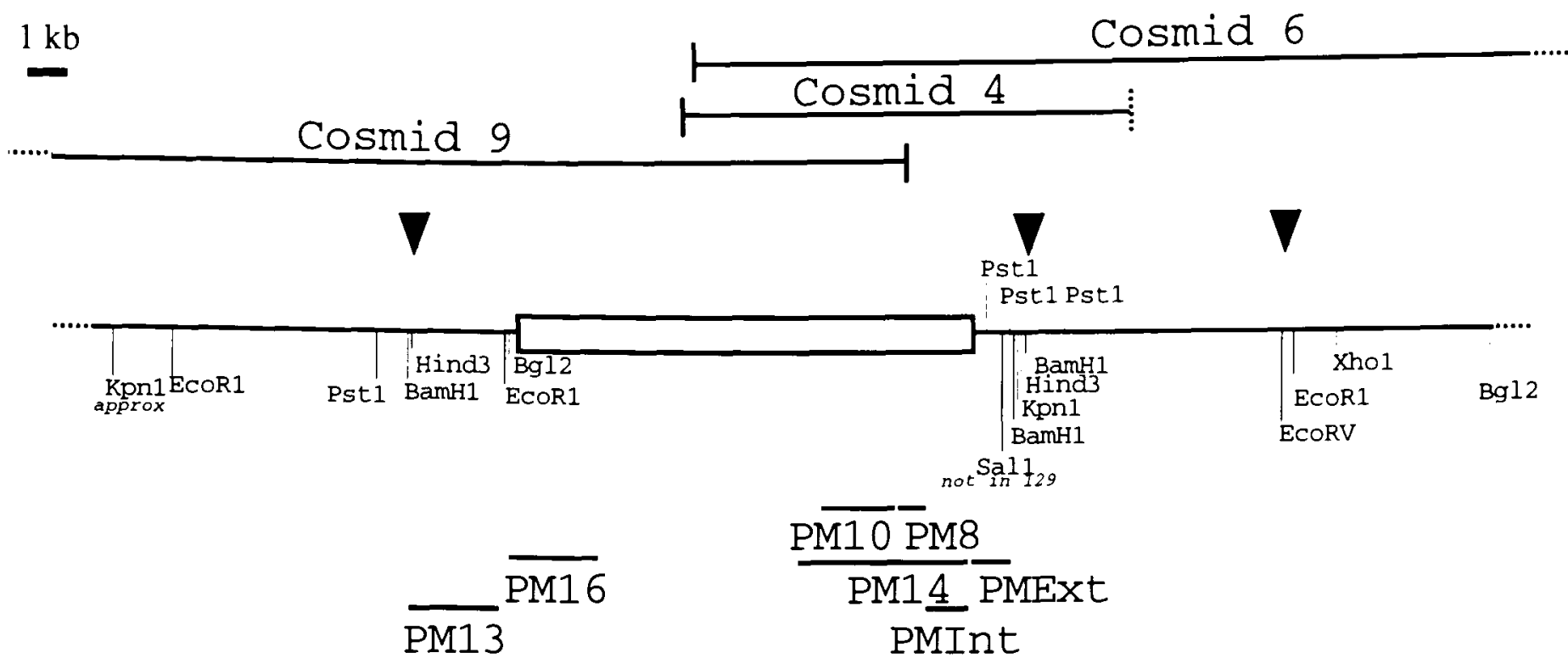

Three cosmids were obtained from a 129/SV library (kindly made available by $\mathrm{Dr} A$. M. Frischauf). In one (cosmid 4) there were two copies of the vector and an additional insert not from the Epo locus. Genomic digests and mapping of the cosmids demonstrated the restriction sites shown. The map has been made working outwards from $\lambda 3$; ; all sites for a particular restriction enzyme are shown as far the outermost site shown for that enzyme; beyond that the map for that enzyme is not complete. The extent of the $\lambda 3$ a clone is shown as an open box; restriction sites in this sequence are not given. Probes external to this sequence are indicated by arrowheads. Plasmid subclones obtained from the cosmid are also indicated below the sequence. Limited sequence from the 5' and 3' ends of the 3 a clone and the immediate flanking region in the cosmid clones is given below. Uncertain bases are underlined. The Sau $3 A$ sites representing the end of Epo sequence in the $\lambda$ clone are shown. This sequence was used for primers for attempted long PCR.

Sequence from $\lambda 3$ a clone ( $3^{\prime}$ end)

\begin{tabular}{|c|c|c|c|c|}
\hline GGGCTCTAGT & TCTGTTCCTT & TTTAAAACCC & TGGACTAAGA & $\begin{array}{r}\text { CAAACATGGA } \\
\text { Sau3A }\end{array}$ \\
\hline CT & TCCTCTGAG & AGCAAAAA & AAGTCCTGC & TGGAACAGA \\
\hline
\end{tabular}

Sequence from 129 cosmid ( $3^{\prime}$ to Xba site) $\mathrm{Xba} 1$

TCTAGAGCT TCCTCTGAGA CAGCAAAAAG CAGCCAGCAG AATGCTTTCC TGGTAAACAT CTIAGAACCA TGTAAAAAGA TGAATGTGGA AAGTCCTGC TGGAACAGAT GAGGGCCCGG GTTTGCATGT GCGATGACAA GAAGGGAAGG AGATGGGT GGTGTGTCCC TGTAATCCCA

\section{Sequence from $\lambda 3 \mathrm{a}$ clone $\left(5^{\prime}\right.$ end $)$}

\section{Sau3A}

GATCTCAGTA TGTAATACAGA CTGCCCTAAAA CTCATTATACA GTCCAGCCTAG TCCCAACACA TGTCAATCCTC $\subseteq$ TGCCTCAGCT TTCCAAGTGAT AGGCTTGCAÄG TGCACCATCC

CACTTTGTTCT T

Sequence from 129 cosmid $\left(5^{\prime}\right.$ to $\left.\lambda 3 \mathrm{a}\right)$ (CT REPEAT) CCTTCCTTCC ATGTAATACA GACTGCCCTA $\operatorname{sau} 3 \mathrm{~A}$

CTGTCTGACA GGATCTCAGT 


\section{Appendix 4}

\section{CHINESE HAMSTER EPO SEQUENCE AND RIBOPROBE EXON 4}

HUMAN GATA AAGCCGTCAG TGGCCTTCGC AGCCTCACCA CTCTGCTTCG GGCTCTGGGA GCCCAG End of exon MOUSE GACA AAGCCATCAG TGGTCTACGT AGCCTCACTT CACTGCTTCG GGTACTGGGA GCTCAG 60bp

RAT TATA GACAAAGCCA TCAGTTACGT AGCCTCACTT CACTGCTTCG GGTGCTGGGA GCTCAG to primer end. CH $\ldots \ldots \ldots \ldots \ldots . \ldots . . \ldots \ldots G C G C$ AGCCTCACCT CCCTGCTCCG GGTGCTGGGA GCCCAG

\section{INTRON 4}

MS

$\mathrm{CH}$

MS

$\mathrm{CH}$

EXON 5

HUMAN

MS

RAT

$\mathrm{CH}$

HUMAN

MS

RAT

$\mathrm{CH}$

HUMAN

MS

RAT

$\mathrm{CH}$
CTC CATTCTGGAC CAGGTCACAT CTTATCTGTA GCCCTCCACA CTCCAGTGAC CCC CTCCTGTCCT TTGTCACGTT TTCATCTGCA GTCCTCAGCA CTGCAGTGAT

ATCCTATTCT CTCTTGACAG End of intron.

Dde 1 Pst 1 ATCCTATTTT CTCTTGACAG

$$
\begin{aligned}
& \text { AAGGA AGCCATCTCC CCTCCAGATG CGGCCTCAGC TGCTCCACTC CGAACAATCA } \\
& \text { AAGGA ATTGATGTCG CCTCCAGATA CCACCCACC TGCTCCACTC CGAACACTCA } \\
& \text { AAGGA ATTGATGTCG CCTCCAGACG CCACCCAAGC CGCTCCACTC CGAACACTCA } \\
& \text { AAGGA ATCGATATTA CCTCCAGATG CTACCCCACC TGCTCCACTC CGAACACTCT } \\
& \text { CTGCTGACAC TTTCCGCAAA CTCTTCCGAG TCTACTCCAA TTTCCTCCGG GGAAAGCTGA } \\
& \text { CAGTGGATAC TTTCTGCAAG CTCTTCCGG TCTACGCCAA CTTCCTCCGG GGGAACTGA } \\
& \text { CAGCGGATAC TTTCTGCAAG CTCTTCCGG TCTACTCCAA CTTCCTCCGG GGAAACTGA } \\
& \text { CGGTGGATAC TTTCTGCAAA CTCTTCCGGG TCTACTCCAA CTTTCTCCGG GGCAAACTGA }
\end{aligned}
$$

AGCTGTACAC AGGGGAGGCC TGCAGGACAG GGGACAGA AGCTGTACAC GGGAGAGGTC TGCAGGAGAG GGGACAGG END OF CODING SEQUENCE. AGCTGTACAC GGGGGAGGCC TGCAGGAGAG GGGACAGG 148 bp to primer end AGTTGTACAC AGG Pst1

Underlined sequence from mouse was used as primers for PCR from Chinese hamster genomic DNA and RT-PCR from hypoxic Chinese hamster kidney RNA. The vector was pSP72 cut with Pstl and EcoRV. cDNA was cloned following Pstl digestion of the PCR fragment. Genomic DNA PCR product was succesfully cloned by partial Pst I digestion of PCR product. $P C R$ cDNA fragment $=208 \mathrm{bp}$; cloned with Pstl and blunt $=200 \mathrm{bp}$. Mouse gDNA product would be $946 \mathrm{bp}$. Genomic chinese hamster product about this length. The genomic DNA clone was in pSP72 as follows: SP6-Xho1-Pvu2-Hind3-Sph1-Pst1-Exon5-Intron4-Exon4-Bgl2T7. A riboprobe template was generated by linearising the genomic clone with Ddel or Bgl2. Chinese hamster hypoxic kidney RNA gave a protected fragment from either template of 140 $b p$, with second band of $60 \mathrm{bp}$ from $\mathrm{Bgl2}$. Full length for Dde1 $=178 \mathrm{bp}$, for Bgl2 approx $1 \mathrm{~kb}$. The Chinese hamster sequence for exon 4 was from the cDNA clone; sequence of intron 4 from 2 clones of genomic PCR product. Sequence of exon 5 from cDNA and 2 genomic clones (identical in this region). 


\title{
Appendix 5
}

\section{3' FLANKING SEQUENCE IN VARIOUS MOUSE STRAINS}

1

51

101

151

201

251

301

351

401

451

501

551

601

651

701

751

801

851

901

951

1001

1051

1101

1151

1201

\begin{abstract}
TGGGGTCCAA TCTTTGTGGG ATCTATGAAG AATACAGGAC CTGGAGAATT 17 bp deletion in 129

TAGGGGGCAA GACGTGAATT CTTTAGATCT CATGGGGGTT TCCCCAGACA

GCACGAGCCC TGCACTGGAC AACAGAGGGT CAAGGAGCCA TAGACAGAAT

GGCCGCTGAC TCTCAGCGTT CATGGGAGGG AAAGGGTTTG ACATTTTGTG

TCTTTTAAAA ATACCACTGG CAACAGCTGA AATCACCAAC CAGACTCCTG

GCTTGTCTCT CTTCATGACT GTACACACCA agcatcagat CTGgGaAaCC AgaggtgGa GGGGTTGGG

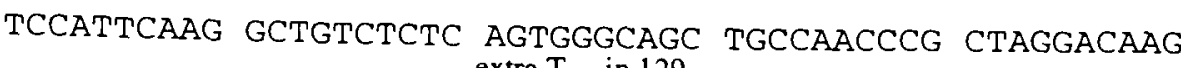

TGCTGAGTGA GCTGgGgCCA CCGTTGAGGA AACAGGAGCC AGTACAGAGG

GGTTCCCCTT TAGGGgTTGGT GGCAATGGGC GACCCTGGTT AATGGATCAT

TATGTCCGTA GTCACTTACC TCAGGACATA AACCCCTTGT GAGTCCATCT

ATCTTTTGTG TCCCGATGGg TATCACTCTG GGAATTTCTC CTTGCCACTA

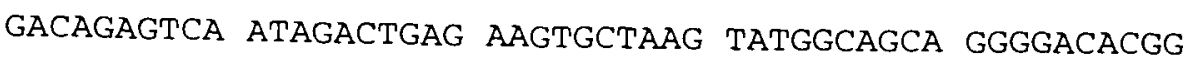

GTGACTCCAA GTCGTTCCTT GGATTCCTTT CCCATAGTGC TTCATGTGGG

AAAGCATGCT TAGGAGAGAG ACCCACACAG CCGACCAGTC AAAGATGGTG

CACAGAGTAC CAGGACCCCA ATCCTGGCTT TTATTCTATG TGGCTGTCAT

TCATTCTGTT CCCAAAACAA GACATTTAGA ATCCAGCCGG AAAGCCTTCA

AAGTCCACTG CAGGAAAAAT TACATGTAGG TTGAAGGAGT TGGGGGCTGG

AGGGAATTTG CTGGGAGTGA GTGTGTGTGT GTGTGTGTGT GTGTGTGTGT

GTGTGTGTGT GTGTGTGTCT ATAATTCAgG TGGgAgGAgG CAGCAAAgGg

GAGGACTCAA AAGAATTTTG GGGGAGgTGA ACTTAGTTCC TGGACTACAG

TGCCCTCTTG AGGTGCAGTT GGGAACTGTT AGGGAACTTT GCCAGCAATC

TCCTTCCTCT GGGATCATCA pBS polylinker

PBSTTATCGA TACCGTGG
\end{abstract}

The sequence shown is Mmery.emrod, which was derived from the $\lambda$ clone used to make the Epo-TAg construct. PCR in which one of the two primers (bases 5-27, 1149-1132) was biotinylated was used to generate template which was denatured and separated using magnetic streptavin beads. The single stranded template was then sequenced (Sequenase 2, USB).The following strains were subjected to analysis of this region; C57BL6, C57BL10, C3H/HeH, CBA, Balb/c, 129/Ola, 101/J, Epo-TAg ${ }^{H}$. Sequence was obtained from bases 50 to 550. Four corrections to mmery. emrod are underlined. Two differences between the A allele (C57BL6\&10, C3H/HeH, CBA, Balb/c) and the B allele (129/Ola, 101/J) were identified and are indicated. No differences between the group of strains with the A allele was identified. Additionally, the 48 bp CA repeat was amplified (primers 1002-1019, 1086-1069) from each strain; the length was identical. 


\section{Appendix 6}

\section{PRINCIPAL PUBLICATIONS ARISING FROM WORK IN THIS THESIS}

Maxwell PH, Pugh CW and Ratcliffe PJ (1993); Inducible operation of the erythropoietin 3 ' enhancer in multiple cell lines: evidence for a widespread oxygen sensing mechanism. Proc Natl Acad Sci USA 90: 2423-2427.

Maxwell PH, Osmond MK, Pugh CW, Heryet A, Nicholls LG, Tan CC, Doe BG, Ferguson DJP, Johnson MH and Ratcliffe PJ (1993); Identification of the renal erythropoietin-producing cells using transgenic mice. Kidney Int 44: 1149-1162.

Maxwell PH, Ferguson DJP, Osmond MK, Pugh CW, Heryet A, Doe BG, Johnson MH and Ratcliffe PJ (1994); Expression of a homologously recombined erythropoietin-SV40 T antigen fusion gene in mouse liver: evidence for erythropoietin production by Ito cells. Blood 84: 1823-1830. 


\section{References}

Abraham NG, Levere RD, Lin JH-C, Beru N, Hermine O and Goldwasser E (1991); Co-regulation of heme oxygenase and erythropoietin genes. J Cell Biochem 47: 43-48.

Adair GM, Nairn RS, Wilson JH, Seidman MM, Brotherman KA, Mackinnon C and Scheerer JB (1989); Targeted homologous recombination at the endogenous adenine phosphoribosyltransferase locus in Chinese hamster cells. Proc Natl Acad Sci USA 86: 4574-4578.

Adams TE, Alpert S and Hanahan D (1987); Non-tolerance and autoantibodies to a transgenic self antigen expressed in pancreatic $\beta$ cells. Nature 325: 223-228.

Adamson JW and Finch CA (1975); Hemoglobin function, oxygen affinity and erythropoietin. Annu Rev Physiol 37: 351-369.

Agron PG, Ditta GS and Helinski DR (1993); Oxygen regulation of nifA transcription in vitro. Proc Natl Acad Sci USA 89: 3506-3510.

Altland PD and Parker M (1955); Effects of hypoxia upon the box turtle. Am J Physiol 180: 421427.

Arendshorst WJ, Finn WF and Gottschalk CW (1975); Pathogenesis of acute renal failure following temporary renal ischaemia in the rat. Circ Res 37: 558-568.

Argyris TS and Trimble ME (1964); The growth promoting effects of damage in the damaged and contralateral kidneys of the mouse. Anat Rec 150: 1-10.

Austyn JM and Gordon S (1981); F4/80, a monoclonal antibody directed specifically against the mouse macrophage. Eur J Immunol 11: 805-815

Bachmann S, Le Hir M and Eckardt K-U (1993); Co-localization of erythropoietin messenger RNA and ecto-5'-nucleotidase immunoreactivity in peritubular cells of rat renal cortex indicates that fibroblasts produce erythropoietin. J Histochem Cytochem 41: 335-341.

Bankir L, Bouby N and Trinh-Trang-Tan M-M (1987); Heterogeneity of nephron anatomy. Kidney Int 31: S25-S39.

Barinaga M (1994); Knockout mice: round two. Science 265: 26-28.

Barnes WM (1994); PCR amplification of up to 35-kb DNA with high fidelity and high yield from $\lambda$ bacteriophage templates. Proc Natl Acad Sci USA 91: 2216-2220.

Baumgärtl H, Leichtweiss H-P, Lübbers DW, Weiss C and Huland H (1972); The oxygen supply of the dog kidney: measurements of intrarenal $\mathrm{pO}_{2}$. Microvasc Res 4: 247-257.

Beato M (1989); Gene regulation by steroid hormones. Cell 56: 335-344.

Beck I, Ramirez S, Weinmann R and Caro J (1991); Enhancer element at the 3'-flanking region controls transcriptional response to hypoxia in the human erythropoietin gene. J Biol Chem 266: 15563-15566.

Benjamin IJ, Kroger B and Williams RS (1990); Activation of heat shock transcription factor by hypoxia in mammalian cells. Proc Natl Acad Sci USA 87: 6263-6267.

Beru N, McDonald J, Lacombe C and Goldwasser E (1986); Expression of the erythropoietin gene. Mol Cell Biol 6: 2571-2575.

Blanchard KL, Acquaviva AM, Galson DL and Bunn HF (1992); Hypoxic induction of the human erythropoietin gene: cooperation between the promoter and enhancer, each of which contains steroid receptor response elements. Mol Cell Biol 12: 5373-5385.

Blumberg A, Keller H and Marti HR (1973); Effect of altitude on erythropoiesis and oxygen affinity in anaemic patients on maintenance dialysis. Eur J Clin Invest 3: 93-97.

Bohman S-O, Sundelin B, Forsum U and Tribukait B (1988); Experimental depletion of different renal interstitial cell populations. Am J Med Sci 295: 252-257.

Bollag RJ, Waldmam S and Liskay RM (1989); Homologous recombination in mammalian cells. Annu Rev Genet 23: 199-225.

Bondurant MC and Koury MJ (1986); Anemia induces accumulation of erythropoietin mRNA in the kidney and liver. Mol Cell Biol 6: 2731-2733. 
Bouwens L, De Blesser P, Vanderkerken K, Geerts B and Wisse E (1992); Liver cell heterogeneity: functions of non-parenchymal cells. Enzyme 46: 155-168.

Brezis M, Rosen S, Silva P and Epstein FH (1984); Renal ischemia: a new perspective. Kidney Int 26: $375-383$.

Brinster RL, Braun RE, Lo D, Avarbock MR, Oram F and Palmiter RD (1989); Targeted correction of a major histocompatibility class II E $\alpha$ gene by DNA microinjected into mouse eggs. Proc Natl Acad Sci USA 86: 7087-7091.

Buratowski N (1994); The basics of basal transcription by RNA polymerase II. Cell 77: 1-3.

Capecchi MR (1989); Altering the genome by homologous recombination. Science 244: 12881292.

Carnot $P$ and Deflandre C (1906); Sur l'activité hémopoiétique du sérum au cours de la régénération du sang. C R Acad Sci Paris 143: 384-386.

Caro J, Brown S, Miller O, Murray T and Erslev AJ (1979); Erythropoietin levels in uremic nephric and anephric patients. J Lab Clin Med 93: 449-458.

Caro J, Hickey J and Erslev AJ (1984); Erythropoietin production by an established kidney proximal tubule cell line (LLCPK1) (abstract). Exp Hematol 12: 357.

Ceci JD, Kovatch RM, Swing DA, Jones JM, Snow CM, Rosenberg MP, Jenkins NA, Copeland NG and Meisler MH (1991); Transgenic mice carrying a murine amylase 2.2/SV40 T antigen fusion gene develop pancreatic acinar cell and stomach carcinomas. Oncogene 6: 323-332.

Chandra M, Clemons GK and McVicar MI (1988); Relation of serum erythropoietin levels to renal excretory function: evidence for lowered set point for erythropoietin production in chronic renal failure. J Pediatr 113: 1015-1021.

Cheng S, Cheng S-Y, Gravitt P and Respess R (1994); Long PCR. Nature 369: 684-685.

Christofori G, Naik P and Hanahan D (1994); A second signal supplied by insulin-like growth factor II in oncogene-induced tumorigenesis. Nature 369: 414-417.

Clemons GK, De Manincor D, Fitzsimmons SL and Garcia JF (1987); Immunoreactive erythropoietin studies in hypoxic rats and the role of the salivary glands. Exp Hematol 15: 18-23.

Cobbold SP, Thierfelder S and Waldmann H (1983); Immunosuppression with monoclonal antibodies. A model to determine the rules for effective serotherapy. Mol Biol Med 1: 285-304.

Costa-Giomi P, Caro J and Weinmann R (1990); Enhancement by hypoxia of human erythropoietin gene transcription in vitro. J Biol Chem 265: 10185-10188.

Cotes PM and Banghham DR (1966); The international reference preparation of erythropoietin. Bull World Health Organ 35: 751.

Cotes PM (1982); Immunoreactive erythropoietin in serum. I. Evidence for the validity of the assay method and the physiological relevance of estimates. Br J Haematol 50: 427-438.

Cotes PM, Doré CJ, Liu Yin JA, Lewis SM, Messinezy M, Pearson TC and Reid C (1986); Determination of serum immunoreactive erythropoietin in the investigation of erythrocytosis. $\mathrm{N}$ Engl J Med 315: 283-287.

Cotes PM, Pippard MJ, Reid CDL, Winearls CG, Oliver DO and Royston JP(1989a); Characterization of the anaemia of chronic renal failure and the mode of its correction by a preparation of human erythropoietin (r-Hu-EPO). An investigation of the pharmacokinetics of intravenous erythropoietin and its effects on erythrokinetics. Q J Med 70: 113-137.

Cotes PM, Tam RC, Reed P and Hellebostad M (1989b); An immunological cross-reactant of erythropoietin in serum which may invalidate EPO radioimmunoassay. Br J Haematol 73: 265-268.

Crawford L and Harlow E (1982); Uniform nomenclature for monoclonal antibodies directed against virus-coded proteins of simian virus 40 and polyoma virus. J Virol 41: 709.

Crossley M and Orkin SH (1993); Regulation of the $\beta$-globin locus. Curr Opin Genet Dev 3: 232237.

Czyzyk-Krzeska MF, Bayliss DA, Lawson EE and Millhorn DE (1992); Regulation of tyrosine hydroxylase gene expression in the rat carotid body by hypoxia. J Neurochem 58: 1538-1546. 
Czyzyk-Krzeska MF, Furnari BA, Lawson EE and Millhorn DE (1994); Hypoxia increases rate of transcription and stability of tyrosine hydroxylase mRNA in phaeochromocytoma (PC12) cells. J Biol Chem 269: 760-764.

Da Silva JL, Lacombe C, Bruneval P, Casadevall N, Leporrier M, Camilleri JP, Bariety J, Tambourin $P$ and Varet B (1990); Tumour cells are the site of erythropoietin synthesis in human renal cancers associated with polycythemia. Blood 75: 577-582.

Davis BD, Thomasson D and Zenser TV (1983); Renal disease profoundly alters cortical interstitial cell function. Kidney Int 23: 485-464.

Dawson TP, Gandhi R, Le Hir M and Kaissling B (1989); Ecto-5'-nucleotidase: localization in rat kidney by light microscopic and immunohistochemical methods. J Histochem Cytochem 37: 39-47.

Deng C and Capecchi MR (1992); Re-examination of gene targeting frequency as a function of the extent of homology between the targeting vector and the target locus. Mol Cell Biol 12: 3365-3371.

Dillon N and Grosveld F (1993); Transcriptional control of multigene loci: multilevel control. Trends Genet 9: 134-137.

Doetschmann T, Gregg RG, Maeda N, Hooper ML, Melton DW, Thompson S and Smithies O (1987); Targeted correction of a mutant HPRT gene in mouse embryonic stem cells. Nature 330: 576-578.

Eckardt K-U, Kurtz A and Bauer C (1989a); Regulation of erythropoietin production is related to proximal tubular function. Am J Physiol 256: F942-F947.

Eckardt K-U, Möllmann M, Neumann R, Brunkhorst R, Burger H-U, Lonnemann G, Scholz H, Keusch G, Buchholz B, Frei U, Bauer C and Kurtz A (1989b); Erythropoietin in polycystic kidneys. J Clin Invest 84: 1160-1166.

Eckardt K-U, Le Hir M, Tan CC, Ratcliffe PJ, Kaissling B and Kurtz A (1992a); Renal innervation plays no role in oxygen dependent-control of erythropoietin mRNA levels. Am J Physiol 263: F925F930.

Eckardt K-U, Ratcliffe PJ, Tan CC, Bauer C and Kurtz A (1992b); Age-dependent expression of the erythropoietin gene in rat liver and kidneys. J Clin Invest 89: 753-760.

Eckardt K-U, Pugh CW, Ratcliffe PJ and Kurtz A (1993); Oxygen dependent expression of the erythropoietin gene in rat hepatocytes in vitro. Pflugers Arch 423: 356-364.

Edmunds ME, Devoy M, Tomson CRV, Krishna U, Clayworth A, Durrant STS, Feehally J and Walls J (1991); Plasma erythropoietin levels and acquired cystic disease of the kidney in patients receiving regular haemodialysis treatment. $\mathrm{Br} \mathrm{J}$ Haematol 78: 275-277.

Erslev AJ (1953); Humoral regulation of red cell production. Blood 8: 349-357.

Erslev AJ, Caro J, Kansu E and Silver R (1980); Renal and extrarenal erythropoietin production in anaemic rats. Br J Haematol 45: 65-72.

Eschbach JW, Egrie JC, Downing MR, Browne JK and Adamson JW (1987); Correction of the anemia of end-stage renal disease with recombinant human erythropoietin: Results of the Phase I and II clinical trial. N Engl J Med 316: 73-78.

Eschbach JW (1989); The anemia of chronic renal failure: Pathophysiology and the effects of recombinant erythropoietin. Kidney Int 35: 134-148.

Fandrey J and Bunn HF (1993); In vivo and in vitro regulation of the erythropoietin mRNA: measurement by competitive polymerase chain reaction. Blood 81: 617-623.

Fanning E (1992); Simian virus 40 large T antigen: the puzzle, the pieces and the emerging picture. J Virol 66: 1289-1293.

Faura J, Ramos J, C. R, English E, Finne P and Finch CA(1969); Effect of altitude on erythropoiesis. Blood 33: 668-676.

Felsenfeld G (1992); Chromatin as an essential part of the transcriptional mechanism. Nature 355: 219-224.

Firth JD, Ebert BL, Pugh CW and Ratcliffe PJ (1994); Oxygen-regulated control elements in the phosphoglycerate kinase 1 and lactate dehydrogenase A genes: similarities with the erythropoietin 3' enhancer. Proc Natl Acad Sci USA 91: 6496-6500 
Fisher JW, Taylor G and Porteous DD (1965); Localisation of erythropoietin in glomeruli of sheep kidney by fluorescent antibody technique. Nature 205: 611-612.

Fried W and Kilbridge T (1969); Effect of testosterone and of cobalt on erythropoietin production by anephric rats. J Lab Clin Med 74: 623-629.

Fried W (1972); The liver as a source of extrarenal erythropoietin production. Blood 40: 671-677.

Fried W, Barone-Varelas J and Barone T (1982); The influence of age and sex on erythropoietin titers in the plasma and tissue homogenates of hypoxic rats. Exp Hematol 10: 472-477.

Fukushima Y, Yanagisawa M, Nakamoto Y and Miura AB (1990); Localization of renal erythropoietin and the role of Ia antigen expression in hypoxic rat glomeruli. Tohoku J Exp Med 160: 129-140.

Galson DL, Tan CC, Ratcliffe PJ and Bunn HF (1993); Comparison of the human and mouse erythropoietin genes shows extensive homology in the flanking regions. Blood 82: 3321-3326.

Garcia JF, Ebbe SN, Hollander L, Cutting HO, Miller ME and Cronkite EP (1982); Radioimmunoassay of erythropoietin: circulating levels in normal and polycythemic human beings. J Lab Clin Med 99: 624-635.

Gilles-Gonzalez MA, Ditta GS and Helinski DR (1991); A haemoprotein with kinase activity encoded by the oxygen sensor of Rhizobium meliloti. Nature 350: 170-172.

Gleadle JM, Ebert BL, Firth JD and Ratcliffe PJ (1995) Oxygen regulated expression of angiogenic growth factors: effects of hypoxia, transition metals and chelating agents. Am J Physiol in press.

Goldberg MA, Glass GA, Cunningham JM and Bunn HF (1987); The regulated expression of erythropoietin by two human hepatoma cell lines. Proc Natl Acad Sci USA 84: 7972-7976.

Goldberg MA, Dunning SP and Bunn HF (1988); Regulation of the erythropoietin gene: evidence that the oxygen sensor is a heme protein. Science 242: 1412-1415.

Goldberg MA, Gaut CC and Bunn HF (1991); Erythropoietin mRNA levels are governed by both the rate of gene transcription and posttranscriptional events. Blood 77: 271-277.

Goldberg MA and Schneider TJ (1994); Similarities between the oxygen-sensing mechanisms regulating the expression of vascular endothelial growth factor and erythropoietin. J Biol Chem 269: 4355-4359.

Gurner AC, Smith J and Cattell V (1987); The origin of Ia antigen-expressing cells in the rat kidney. Am J Pathol 127: 342-348.

Gurney EG, Harrison RO and Fenno J (1980); Monoclonal antibodies against simian virus $40 \mathrm{~T}$ antigens: evidence for distinct subclasses of large $T$ antigen and for similarities among nonviral $T$ antigens. J Virol 34: 752-763.

Hanahan D (1985); Heritable formation of pancreatic $\beta$-cell tumours in transgenic mice expressing recombinant insulin/simian virus 40 oncogenes. Nature 315: 115-122.

Harlow E, Crawford LV, Pim DC and Williamson NM (1981); Monoclonal antibodies specific for simian virus 40 tumor antigens. J Virol 39: 861-869.

Hart DNJ and Fabre JW (1981); Demonstration and characterization of Ia-positive dendritic cells in the interstitial connective tissues of rat heart and other tissues, but not brain. J Exp Med 153: 347361.

Hasty P, Ramirez SR, Krumlauf R and Bradley A (1991); Introduction of a subtle mutation into the Hox-2.6 locus in embryonic stem cells. Nature 350: 243-246.

Imagawa S, Goldberg MA, Doweiko J and Bunn HF (1991); Regulatory elements of the erythropoietin gene. Blood 77: 278-285.

Jackson IJ (1991); A reappraisal of non-concensus mRNA splice sites. Nucleic Acids Res 19: 37953798.

Jacobs K, Shoemaker C, Rudersdorf R, Neill SD, Kaufman RJ, Mufson A, Seehra J, Jones SS, Hewick R, Fritsch EF, Kawakita M, Shimizu T and Miyake T (1985); Isolation and characterization of genomic and cDNA clones of human erythropoietin. Nature 313: 806-810.

Jacobson LO, Goldwasser E, Fried W and Plzak L (1957); Role of the kidney in erythropoiesis. Nature 179: 633-634. 
Jat PS and Sharp PA (1986); Large T antigens of simian virus 40 and polyomavirus efficiently establish primary fibroblasts. J Virol 59: 746-750.

Jat PS, Noble MD, Ataliotis P, Tanaka Y, Yannoutsos N, Larsen L and Kioussis D (1991); Direct derivation of conditionally immortalised cell lines from an $\mathrm{H}-2 \mathrm{~K}^{\mathrm{b}}$-tsA 58 transgenic mouse. Proc Natl Acad Sci USA 88: 5096-5100.

Jelkmann W, Huwiler A, Fandrey J and Pfeilschifter J (1991); Inhibition of erythropoietin production by phorbol ester is associated with down-regulation of protein kinase $\mathrm{C}$-alpha isoenzyme in hepatoma cells. Biochem Biophys Res Commun 179: 1441-1448.

Jelkmann W (1992); Erythropoietin: Structure, control of production, and function. Physiol Rev 72: 449-489.

Jordan HE (1933); The evolution of blood-forming tissues. Q Rev Biol 8: 58-76.

Jungermann K and Katz N (1989); Functional specialization of different hepatocyte populations. Physiol Rev 69: 708-764.

Kaissling B, Spiess S, Rinne B and Le HM (1993); Effects of anemia on morphology of rat renal cortex. Am J Physiol 264: F608-F617.

Kaissling B and Le Hir M (1994); Characterization and distribution of interstitial cell types in the renal cortex of rats. Kidney Int 45: 709-720.

Kickler TS and Spivak JL (1988); Effect of repeated whole blood donations on serum immunoreactive erythropoietin levels in autologous donors. JAMA 260: 65-67.

Kietzmann T, Schmidt H, Probst I and Jungermann K (1992); Modulation of the glucagon-dependent activation of the phosphoenolpyruvate carboxykinase gene by oxygen in rat hepatocyte cultures. Evidence for a heme protein as oxygen sensor. FEBS Lett 311: 251-255.

Knighton DR, Hunt TK, Scheuenstuhl H, Halliday BJ, Webb Z and Banda MJ (1983); Oxygen tension regulates the expression of angiogenesis factor by macrophages. Science 221: 1283-1285.

Kominami N, Lowrie EG, Ianhez LE, Skaren A, Hampers CL, Merrill JP and Lange RD (1971); The effect of total nephrectomy on hematopoiesis in patients undergoing chronic haemodialysis. $\mathrm{J}$ Lab Clin Med 78: 524-532.

Kourembanas S, Hannan RL and Faller DV (1990); Oxygen tension regulates the expression of the platelet-derived growth factor - B chain gene in human endothelial cells. J Clin Invest 86: 670-674.

Kourembanas S, Marsden PA, McQuillan LP and Faller DV (1991); Hypoxia induces endothelin gene expression and secretion in cultured human endothelium. J Clin Invest 88: 1054-1057.

Koury MJ, Bondurant MC, Graber SE and Sawyer ST (1988a); Erythropoietin messenger RNA levels in developing mice and transfer of ${ }^{125}$ I-erythropoietin by the placenta. J Clin Invest 82: 154159.

Koury ST, Bondurant MC and Koury MJ (1988b); Localization of erythropoietin synthesizing cells in murine kidneys by in situ hybridization. Blood 71: 524-527.

Koury ST, Koury MJ, Bondurant MC, Caro J and Graber SE (1989); Quantitation of erythropoietinproducing cells in kidneys of mice by in situ hybridization: correlation with hematocrit, renal erythropoietin mRNA and serum erythropoietin concentration. Blood 74: 645-651.

Koury ST, Bondurant MC, Koury MJ and Semenza GL (1991); Localization of cells producing erythropoietin in murine liver by in situ hybridization. Blood 77: 2497-2503.

Krantz SB and Jacobson LO (1970) Erythropoietin and the regulation of erythropoiesis. Chicago, University of Chicago Press.

Krantz SB (1991); Erythropoietin. Blood 77: 419-434.

Kriz W (1987); A periarterial pathway for the distribution of renin. Kidney Int 31: S51-S56.

Krystal G (1983); A simple microassay for erythropoietin based on ${ }^{3} \mathrm{H}$-thymidine incorporation into spleen cells from phenylhydrazine treated mice. Exp Hematol 11: 649-660.

Kurtz A, Jelkmann W, Sinowatz F and Bauer C (1983); Renal mesangial cell cultures as a model for study of erythropoietin production. Proc Natl Acad Sci USA 80: 4008-4011. Kurtz A, Eckardt K-U, Tannahill L and Bauer C (1988); Regulation of erythropoietin production.
Contrib Nephrol 66: 1-16. 
Kurtz A, Eckardt KU, Neumann R, Kaissling B, Le Hir M and Bauer C (1989); Site of erythropoietin formation. Contrib Nephrol 76: 14-23.

Kurtz A, Eckardt K-U, Pugh C, Corvol P, Fabbro D and Ratcliffe P (1992); Phorbol ester inhibits erythropoietin production in human hepatoma cells (Hep G2). Am J Physiol 262: C1204-C1210.

Lacombe C, Da Silva J-L, Bruneval P, Fournier J-G, Wendling F, Casadevall N, Camilleri J-P, Bariety J, Varet B and Tambourin P (1988); Peritubular cells are the site of erythropoietin synthesis in the murine hypoxic kidney. J Clin Invest 81: 620-623.

Lacombe C, Da Silva J-L, Bruneval P, Chesne C, Guilloza A, Camilleri J-P, Bariety J, Varet B and Tambourin P (1991) Expression of the erythropoietin gene in the kidney and the liver of the anaemic mouse. Response and adaptation to hypoxia: organ to organelle. Oxford University Press. 69-77.

Le Hir M, Eckardt K-U and Kaissling B (1989); Anemia induces 5'-nucleotidase in fibroblasts of cortical labyrinth of rat kidney. Renal Physiol Biochem 12: 313-319.

Le Hir M and Kaissling B (1989); Distribution of 5'-nucleotidase in the renal interstitium of the rat. Cell Tissue Res 258: 177-182.

Ledent C, Dumont J, Vassart G and Parmentier M (1991); Thyroid adenocarcinomas secondary to tissue-specific expression of simian virus-40 large T-antigen in transgenic mice. Endocrinology 129: 1391-1401.

Lee SH, Crocker P and Gordon S (1986); Macrophage plasma membrane and secretory properties in murine malaria. Effects of Plasmodium yoelii blood-stage infection on macrophages in liver, spleen, and blood. J Exp Med 163: 54-74.

Lemley KV and Kriz W (1991); Anatomy of the renal interstitium. Kidney Int 39: 370-381.

Lin F-K, Suggs S, Lin C-H, Browne JK, Smalling R, Egrie JC, Chen KK, Fox GM, Martin F, Stabinsky Z, Badrawi SM, Lai P-H and Goldwasser E (1985); Cloning and expression of the human erythropoietin gene. Proc Natl Acad Sci USA 82: 7580-7584.

Lin F-K, Lin C-H, Lai P-H, Browne JK, Egrie JC, Smalling R, Fox GM, Chen KK, Castro M and Suggs S (1986); Monkey erythropoietin gene: cloning, expression and comparison with the human erythropoietin gene. Gene 44: 201-209.

Lorentz A, Jendrissek A, Eckardt K-U, Schipplick M, Osswald PM and Kurtz A (1991); Serial immunoreactive erythropoietin levels in autologous blood donors. Transfusion 31: 650-654.

Lyon MF, Glenister PH, Loutit JF and Peters J (1983); Dominant haemolytic anaemia. Mouse Newsletter 68: 68.

Madan A and Curtin P (1993); A 24 base pair sequence 3' to the human erythropoietin gene contains a hypoxia-responsive transcriptional enhancer. Proc Natl Acad Sci USA 90: 3928-3932.

Mansour SL, Thomas KR and Capecchi MR (1988); Disruption of the proto-oncogene int-2 in mouse embryo-derived stem cells: a general strategy for targeting mutations to non-selectable genes. Nature 336: 348-352.

Masuda S, Okana M, Yamagishi K, Nagao M, Ueda M and Sasaki R (1994); A novel site of erythropoietin production. J Biol Chem 269: 19488-19493.

Maxwell AP, Lappin TRJ, Johnston CF, Bridges JM and McGeown MG (1990); Erythropoietin production in kidney tubular cells. Br J Haematol 74: 535-539.

McDonald JD, Lin F-K and Goldwasser E (1986); Cloning, sequencing, and evolutionary analysis of the mouse erythropoietin gene. Mol Cell Biol 6: 842-848.

McGonigle RJS, Wallin JD, Shadduck RK and Fisher JW (1984); Erythropoietin deficiency and inhibition of erythropoiesis in renal insufficiency. Kidney Int 25: 437-444.

Melton DA, Krieg PA, Rebagliati MR, Maniatis T, Zinn K and Green MR (1984); Efficient in vitro synthesis of biological active RNA and RNA hybridization probes from plasmids containing a bacteriophage SP6 promoter. Nucleic Acids Res 12: 7035-7056.

Miescher F (1893); Über die Beziehugen zwischen Meereshöhe und Beschaffenheit des Blutes. Cor Bl Schweiz Aerztebl 23: 809.

Milledge JS and Cotes PM (1985); Serum erythropoietin in humans at high altitude and its relation to plasma renin. J Appl Physiol 59: 360-364. 
Minchenko A, Bauer T, Salceda S and Caro J (1994a); Hypoxic stimulation of vascular endothelial growth factor expression in vitro and in vivo. Lab Invest in press.

Minchenko A, Salceda S, Bauer T and Caro J (1994b); Hypoxia regulatory elements of the human vascular endothelial growth factor gene. Cell Mol Biol Res 40: 35-39.

Mitchell PJ and Tjian R (1989); Transcriptional regulation in mammalian cells by sequence-specific DNA binding proteins. Science 245: 371-378.

Miyake T, Kung KH and Goldwasser E (1977); Purification of human erythropoietin. J Biol Chem 252: 5558-5564.

Monson EK, Weinstein M, Ditta GS and Helinski DR (1992); The FixL protein of rhizobium meliloti can be separated into a heme-binding oxygen-sensing domain and a functional C-terminal kinase domain. Proc Natl Acad Sci USA 89: 4280-4284.

Montag AG, Oka T, Baek KH, Choi CS, Jay G and Agarwal K (1993); Tumors in hepatobiliary tract and panreatic islet tissues of transgenic mice harboring gastrin simian virus 40 large tumor antigen fusion gene. Proc Natl Acad Sci USA 90: 6696-6700.

Montesano R, Matsumoto K, Nakamura T and Orci L (1991); Identification of a fibroblast-derived epithelial morphogen as hepatocyte growth factor. Cell 67: 901-908.

Naets JP and Wittek M (1968); Presence of erythropoietin in the plasma of one anephric patient. Blood 31: 249-251.

Nagle RB, Kneiser MR, Bulger RE and Benditt EP (1973); Induction of smooth muscle characteristics in renal interstitial fibroblasts during obstructive nephropathy. Lab Invest 29: 422-427.

Nagle RB, Evans LW and Reynolds DG (1975); Contractility of renal cortex following complete ureteral obstruction. Proc Soc Exp Biol Med 148: 611-614.

Nasr-Esfahani M, Johnson MH and Aitken RJ (1990); The effect of iron and iron chelators on the in vitro block to development of the mouse preimplantation embryo: BAT6 a new medium for improved culture of mouse embryos in vitro. Hum Reprod 5: 997-1003.

Naughton BA, Kaplan SM, Brudowski AJ, Roy M, Gordon AS and Piliero SJ (1977); Hepatic regeneration and erythropoietin production in the rat. Science 196: 301-302.

Necas E and Neuwirt J (1969); Lack of erythropoietin in plasma of anaemic rats exposed to hyperbaric oxygen. Life Sci 8: 1221-1228.

Necas E and Neuwirt J (1971) Cobalt and erythropoietin production. The regulation of erythropoiesis and haemoglobin synthesis. Prague, Universita Karlova. 91-97.

Necas E and Neuwirt J (1972); The effect of inhibitors of energy metabolism on erythropoietin production. J Lab Clin Med 79: 388-396.

Necas E and Thorling EB (1972); Unresponsiveness of erythropoietin-producing cells to cyanide. Am J Physiol 222: 1187-1190.

Necas (1994); Erythropoietin gene expression in a hydronephrotic kidney (abstract). Clin Investig 72: B5.

Ornitz DM, Hammer RE, Messing A, Palmiter RD and Brinster RL (1987); Pancreatic neoplasia induced by SV40 T antigen expression in acinar cells of transgenic mice. Science 238: 188-192.

Osnes S (1958); An erythropoietic factor produced in the kidney. BMJ 2: 1387.

Pabo CO and Sauer RT (1992); Transcription factors: Structural families and principles of DNA recognition. Annu Rev Biochem 61: 1053-1095.

Pagel H, Jelkmann W and Weiss C (1989); Oxygen supply to the kidneys and the production of erythropoietin. Respir Physiol 77: 111-118.

Pagel H, Jelkmann W and Weiss C (1991); Isolated serum-free perfused rat kidneys release immunoreactive erythropoietin in response to hypoxia. Endocrinology 128: 2633-2638.

Palmiter RD and Brinster RL (1986); Germ-line transformation of mice. Annu Rev Genet 20: 465499.

Paul P, Rothmann SA, McMahon JT and Gordon AS (1984); Erythropoietin secretion by isolated rat Kupffer cells. Exp Hematol 12: 825-830. 
Plzak L, Fried W, Jacobson LO and Bethard WF (1955); Demonstration of stimulation of erythropoiesis by plasma from anemic rats using Fe ${ }^{59}$. J Lab Clin Med 46: 671-678.

Potter CG, Tan CC and Ratcliffe PJ (1991); Quantification of 32P-labelled samples in gel fragments using the flat-bed liquid scintillation counter. Anal Biochem 197: 121-124.

Pretlow TP, Lapinsky AS, Flowers LC, Grane RW and Pretlow TG (1987); Enzymatic histochemistry of mouse kidney in plastic. J Histochem Cytochem 35: 483-487.

Pugh CW, Tan CC, Jones RW and Ratcliffe PJ (1991); Functional analysis of an oxygen-regulated transcriptional enhancer lying 3 ' to the mouse erythropoietin gene. Proc Natl Acad Sci USA 88: 10553-10557.

Pugh CW, Ebert BL, Ebrahim O and Ratcliffe PJ (1993); Characterisation of functional domains within the mouse erythropoietin 3'enhancer conveying oxygen-regulated responses in different cell lines. Biochim Biophys Acta 1217: 297-306.

Raja KB, Pippard MJ, Simpson RJ and Peters TJ (1986); Relationship between erythropoiesis and the enhanced intestinal uptake of ferric iron in hypoxia in the mouse. Br J Haematol 64: 587-593.

Ramadori G (1991); The stellate cell (Ito-cell, fat-storing cell, lipocyte, perisinusoidal cell) of the liver. Virchows Arch B Cell Pathol 61: 147-158.

Ratcliffe PJ, Jones RW, Phillips RE, Nicholls LG and Bell JI (1990); Oxygen-dependent modulation of erythropoietin mRNA levels in isolated rat kidneys studied by RNAase protection. J Exp Med 172: $657-660$.

Reissmann KR (1950); Studies on the mechanism of erythropoietic stimulation in parabiotic rats during hypoxia. Blood 5: 372-379.

Reynolds RK, Hoekzema GS, Vogel J, Hinrichs SH and Jay G (1988); Multiple endocrine neoplasia induced by the promiscuous expression of a viral oncogene. Proc Natl Acad Sci USA 85: 31353139.

Rich IN, Vogt C and Pentz S (1988); Erythropoietin gene expression in vitro and in vivo detected by in situ hybridisation. Blood Cells 14: 505-520.

Rodemann HP and Müller GA (1990); Abnormal growth and clonal proliferation of fibroblasts derived from kidneys with interstitial fibrosis. Proc Soc Exp Biol Med 195: 57-63.

Rodemann HP and Müller GA (1991); Characterization of human renal fibroblasts in health and disease: II. In vitro growth, differentiation, and collagen synthesis of fibroblasts from kidneys with interstitial fibrosis. Am J Kidney Dis 6: 684-686.

Rodemann HP, Müller GA, Knecht A, Norman JT, Fine LG (1991); Fibroblasts of rabbit kidney in culture. I. Characterization and identification of cell-specific markers. Am J Physiol 261: F283F291.

Roesler WJ, Vandenbark GR and Hanson RW (1988); Cyclic AMP and the induction of eukaryotic gene transcription. J Biol Chem 263: 9063-9066.

Rondon IJ, MacMillan LA, Beckman BS, Goldberg MA, Schneider T, Bunn HF and Malter JS (1991); Hypoxia up-regulates the activity of a novel erythropoietin mRNA binding protein. J Biol Chem 266: 16594-16598.

Rosse WF, Waldmann T and Hull E (1963); Factors stimulating erythropoiesis in frogs. Blood 22: 66-72.

Rosse WF and Waldmann TA (1966); Factors controlling erythropoiesis in birds. Blood 27: 654661.

Russell ES and Meier H (1966) Constitutional Diseases. Biology of the laboratory mouse. New York, Dover. 571-587.

Schirmacher P, Geerts A, Pietrangelo A and Rogler CE (1992); Hepatocyte growth factor/ hepatopoietin A is expressed in fat-storing cells from rat liver but not myofibroblast-like cells derived from fat-storing cells. Hepatology 15: 5-11.

Schlesinger (1990); Heat shock proteins. J Biol Chem 265: 12111-12114.

Schmid TC, Loffing J, Le Hir M and Kaissling B; Distribution of ecto-5'-nucleotidase in the rat liver: effect of anaemia. Submitted to Histochemistry. 
Schmitt RM, Bruyns E and Snodgrass HR (1991); Haematopoietic development of embryonic stem cells in vitro: cytokine and receptor gene expression. Genes Dev 5: 728-740.

Scholz H, Baier W, Ratcliffe P, Eckardt K, Zapf J, Kurtz A and Bauer C (1992); Insulin-like growth factors decrease oxygen-regulated erythropoietin production by human hepatoma cells. Am J Physiol 263: C474-C479.

Schooley JC and Garcia JF (1962); Immunologic studies on the mechanism of action of erythropoietin. Proc Soc Exp Biol Med 110: 636-641.

Schooley JC and Mahlmann LJ (1972a); Erythropoietin production in the anephric rat. I. Relationship between nephrectomy, time of hypoxic exposure and erythropoietin production. Blood 39: 31-38.

Schooley JC and Mahlmann LJ (1972b); Evidence for the de novo synthesis of erythropoietin in hypoxic rats. Blood 40: 662-670.

Schreiner GF, Harris KPG, Purkerson ML and Klahr S (1988); Immunological aspects of acute ureteral obstruction: immune cell infiltrate in the kidney. Kidney Int 34: 487-493.

Schurek H-J and Kriz W (1985); Morphologic and functional evidence for oxygen deficiency in the isolated perfused rat kidney. Lab Invest 53: 145-155.

Schurek HJ, Jost U, Baumgartl H, Bertram H and Heckmann U (1990); Evidence for a preglomerular oxygen diffusion shunt in rat renal cortex. Am J Physiol 259: F910-F915.

Schuster SJ, Wilson JH, Erslev AJ and Caro J (1987); Physiologic regulation and tissue localization of renal erythropoietin messenger RNA. Blood 70: 316-318.

Schuster SJ, Badiavas EV, Costa-Giomi P, Weinmann R, Erslev AJ and Caro J (1989); Stimulation of erythropoietin gene transcription during hypoxia and cobalt exposure. Blood 73: 13-16.

Schuster SJ, Koury ST, Bohrer M, Salceda S and Caro J (1992); Cellular sites of extrarenal and renal erythropoietin production in anaemic rats. Br J Haematol 81: 153-159.

Sell C, Rubini M, Liu J-P, Efstratiadis A and Baserga R (1993); Simian virus 40 large tumor antigen is unable to transform mouse embryonic fibroblasts lacking type 1 insulin-like growth factor receptor. Proc Natl Acad Sci USA 90: 11217-11221.

Semenza GL, Traystman MD, Gearhart JD and Antonarakis SE (1989); Polycythaemia in transgenic mice expressing the human erythropoietin gene. Proc Natl Acad Sci USA 86: 2301-2305.

Semenza GL, Dureza RC, Traystman MD, Gearhart JD and Antonarakis SE (1990); Human erythropoietin gene expression in transgenic mice: multiple transcription initiation sites and cisacting regulatory elements. Mol Cell Biol 10: 930-938.

Semenza GL, Koury ST, Nejfelt MK, Gearhart JD and Antonarakis SE (1991a); Cell type-specific and hypoxia-inducible expression of the human erythropoietin gene in transgenic mice. Proc Natl Acad Sci USA 88: 8725-8729.

Semenza GL, Nejfelt MK, Chi SM and Antonarakis SE (1991b); Hypoxia-inducible nuclear factors bind to an enhancer element located 3' to the human erythropoietin gene. Proc Natl Acad Sci USA 88: $5680-5684$.

Semenza GL and Wang GL (1992); A nuclear factor induced by hypoxia via de novo protein synthesis binds to the human erythropoietin gene enhancer at a site required for transcriptional activation. Mol Cell Biol 12: 5447-5454.

Semenza GL, Roth PH, Fang H-M and Wang GL (1994); Transcriptional regulation of genes encoding glycolytic enzymes by hypoxia-inducible factor 1. J Biol Chem 269: 23757-23763.

Sherwood JB and Goldwasser E (1979); A radioimmunoassay for erythropoietin. Blood 54: 885893.

Sherwood JB and Shouval D (1986); Continuous production of erythropoietin by an established human renal carcinoma cell line: development of the cell line. Proc Natl Acad Sci USA 83: 165169.

Shoemaker CB and Mitsock LD (1986); Murine erythropoietin gene: cloning, expression and human gene homology. Mol Cell Biol 6: 849-858.

Shweiki D, Itin A, Soffer D and Keshet E (1992); Vascular endothelial growth factor induced by hypoxia may mediate hypoxia-initiated angiogenesis. Nature 359: 843-845. 
Simon P, Meyrier A, Tanquerel T and Ang K-S (1980); Improvement of anaemia in haemodialysed patients after viral or toxic hepatic cytolysis. BMJ 280: 892-894.

Singh A, Eckardt K-U, Zimmermann A, Gotz KH, Hamann M, Ratcliffe PJ, Kurtz A and Reinhart WH (1993); Increased plasma viscosity as a reason for inappropriate erythropoeitin formation. $\mathbf{J}$ Clin Invest 91: 251-256.

Skalknik DG, Dorfman DM, Williams DA and Orkin SH (1991); Restriction of neuroblastoma to the prostate gland in transgenic mice. Mol Cell Biol 11: 4518-4527.

Slater EC (1967); Application of inhibitors and uncouplers for the study of oxidative phosphorylation. Methods Enzymol. 10: 48-57.

Song K-Y, Schwartz F, Maeda N, Smithies O and Kucherlapati R (1987); Accurate modification of a chromosonal plasmid by homologous recombination in human cells. Proc Natl Acad Sci USA 84: 6820-6824.

Springer T, Galfrè G, Secher DS and Milstein C (1978); Monoclonal xenogeneic antibodies to murine cell surface antigens: identification of novel leukocyte differentiation antigens. Eur J Immunol 8: 539-551.

Stahl H, Droge P and Knippers R (1986); DNA helicase activity of SV40 large tumor antigen. EMBO J 5: 1939-1944.

Steinman RM, Nogueira N, Witmer MD, Tydings JD and Mellman IS (1980); Lymphokine enhances the expression and synthesis of Ia antigens on cultured mouse peritoneal macrophages. J Exp Med 152: $1248-1261$.

Stohlmann J, Rath CE and Rose JC (1954); Evidence for a humoral regulator of erythropoiesis. Blood 9: 721-733.

Suzuki T and Sasaki R (1990); Immunocytochemical demonstration of erythropoietin immunoreactivity in peritubular endothelial cells of the anemic mouse kidney. Arch Histol Cytol 53: $121-124$.

Tam RC, Bedwell J, Cotes PM and Reed PJ (1989); Sexual dimorphism of Epo-degrading activity in mouse submaxillary gland extracts. Exp Hematol 17: 160-163.

Tan CC (1992). Regulation of erythropoietin messenger RNA. PhD thesis. National University of Singapore.

Tan CC and Ratcliffe PJ (1991); Effect of inhibitors of oxidative phosphorylation on erythropoietin mRNA in isolated perfused rat kidneys. Am J Physiol 261: F982-F987.

Tan CC, Eckardt K-U and Ratcliffe PJ (1991); Organ distribution of erythropoietin messenger RNA in normal and uremic rats. Kidney Int 40: 69-76.

Tan CC, Eckardt K-U, Firth JD and Ratcliffe PJ (1992); Feedback modulation of renal and hepatic erythropoietin mRNA in response to graded anemia and hypoxia. Am J Physiol 263: F474-481.

Tate PH and Bird AP (1993); Effects of DNA methylation on DNA-binding proteins and gene expression. Curr Opin Genet Dev 3: 226-231.

Te Riele H, Maandag ER and Berns A (1992); Highly efficient gene targeting in embryonic stem cells through homologous recombination with isogenic DNA constructs. Proc Natl Acad Sci USA 89: $5128-5132$.

Thomas KR, Folger KR and Capecchi MR (1986); High frequency targeting of genes to specific sites in the mammalian genome. Cell 44: 419-428.

Tijan R (1981); T antigen binding and the control of SV40 gene expression. Cell 26: 1-2.

Tijan R and Maniatis T (1994); Transcriptional activation: a complex puzzle with few easy pieces. Cell 77: 5-8.

Trimble M, Caro J, Talalla A and Brain M (1991); Secondary erythrocytosis due to a cerebellar hemangioblastoma: demonstration of erythropoietin mRNA in the tumor. Blood 78: 599-601.

Tsuchiya T, Ochiai H, Imajob-Ohmi S, Ueda M, Suda T, Nakamura M and Kanegasaki S (1992); In vitro reconstitution of an erythropoietin gene transcription system using its 5'-flanking sequence and a nuclear extract from anemic kidney. Biochem Biophys Res Commun 182: 137-143.

Tsuchiya T, Ueda M, Ochiai H, Imajoh-Ohmi S and Kanegasaki S (1993); Erythropoietin 5'-flanking sequence-binding protein induced during hypoxia and cobalt exposure. J Biochem 113: 395-400. 
Unkeless JC (1979); Characterization of a monoclonal antibody directed against mouse macrophages and lymphocyte Fc receptors. J Exp Med 150: 580.

Valancius V and Smithies O (1991); Testing an "in-out" targeting procedure for making subtle genomic modifications in mouse embryonic stem cells. Mol Cell Biol 11: 1402-1408.

Van Vliet E, Melis M and Van Ewijk W (1984); Monoclonal antibodies to stromal cell types of the mouse thymus. Eur J Immunol 14: 524-529.

Vecchi A, Garlanda C, Lampugnani MG, Resnati M, Matteucci C, Stoppacciaro A, Schnurch H, Risau W, Ruco L and Mantovani A (1994); Monoclonal antibodies specific for endothelial cells of mouse blood vessels. Their application in the identification of adult and embryonic endothelium. Eur J Cell Biol 63: 247-254.

Wang GL and Semenza GL (1993a); Characterization of hypoxia-inducible factor 1 and regulation of DNA binding activity by hypoxia. J Biol Chem 268: 21513-21518.

Wang GL and Semenza GL (1993b); Desferrioxamine induces erythropoietin gene expression and Hypoxia-Inducible Factor 1 DNA-binding activity: implications for models of hypoxia signal transduction. Blood 82: 3610-3615.

Wang GL and Semenza GL (1993c); General involvement of hypoxia-inducible factor 1 in transcriptional response to hypoxia. Proc Natl Acad Sci U S A 90: 4304-8.

Webster KA (1987); Regulation of glycolytic enzyme RNA transcriptional rates by oxygen availability in skeletal muscle cells. Mol Cell Biochem 77: 19-28.

Webster KA, Discher DJ and Bishopric NH (1993); Induction and nuclear accumulation of Fos and Jun proto-oncogenes in hypoxic cardiac myocytes. J Biol Chem 268: 16852-16858.

Wesson LG (1989); Compensatory growth and other growth responses of the kidney. Nephron 51: 149-184.

Westerveld A, Visser RPLS, Khan PM and Bootsma D (1971); Loss of human genetic markers in man-Chinese hamster somatic cell hybrids. Nature New Biol 234: 20-24.

Widelitz RB, Magun BE and Gerner EW (1986); Effects of cycloheximide on thermotolerance expression, heat shock protein synthesis, and heat shock protein mRNA accumulation in rat fibroblasts. Mol Cell Biol 6: 1088-1094.

Winearls CG, Oliver DO, Pippard MJ, Reid C, Downing MR and Cotes PM (1986); Effect of human erythropoietin derived from recombinant DNA on the anaemia of patients maintained by chronic haemodialysis. Lancet 2: 1175-1178.

Wölfle D and Jungermann K (1985); Long-term effects of physiological oxygen concentrations on glycolysis and gluconeogenesis in hepatocyte cultures. Eur J Biochem 151: 299-303.

Woolf AS, Kolatsi M, Gherardi E, Andermarcher E, Fine LG, Jat PS and Noble MP (1993); Isolation and characterisation of epithelial and fibroblast kidney precursor cells from $\mathrm{H}-2 \mathrm{~K}^{\mathrm{b}}$-tsA58 transgenic mice (abstract). J Am Soc Nephrol 4: 480.

Yachou A-K, Renaudie F, Guenet J-L, Simon-Chazottes D, Jones R, Grandchamp B and Beaumont C (1991); Mouse ferritin $\mathrm{H}$ multigene family is polymorphic and contains a single multiallelic functional gene located on chromosone 19. Genomics 10: 531-538.

Yokoi Y, Namahisa T, Kuroda H, Komatsu I, Miyazaki A, Watanabe S and Usui K (1984); Immunocytochemical detection of desmin in fat-storing cells (Ito cells). Hepatology 4: 709-714.

Zanjani ED, Yu M-L, Perlmutter A and Gordon AS (1969); Humoral factors influencing erythropoiesis in the fish (blue gourami-Trichogaster trichopterus). Blood 33: 573-581.

Zanjani ED, Peterson EN, Gordon AS and Wasserman LR (1974); Erythropoietin production in the fetus: role of the kidney and maternal anemia. J Lab Clin Med 83: 281-287.

Zanjani ED, Poster J, Burlington H, Mann LI and Wasserman LR (1977); Liver as the primary site of erythropoietin formation in the fetus. J Lab Clin Med 89: 640-644.

Zanjani ED, Acensao JI, McGlave PB, Banisadre M and Ash RC (1981); Studies on the liver to kidney switch of Epo production. Clinical Investigation 67: 1183-1188.

Zimmer A and Gruss P (1989); Production of chimaeric mice using embryonic stem (ES) cells carrying a homeobox Hox 1.1 allele mutated by homologous recombination. Nature 338: 150-153. 